\title{
DESENVOLVIMENTO DE MODELOS NUMÉRICOS PARA ANÁLISE DE PROBLEMAS DE INTERAÇÃO DE DOMÍNIOS BIDIMENSIONAIS.
}

Tese apresentada à Escola de Engenharia de São Carlos, Universidade de São Paulo, para a obtenção do título de Doutor em Engenharia de Estruturas

Área de Concentração: Métodos Numéricos Orientador: Prof. Tit. Wilson Sérgio Venturini

SÃO CARLOS

2007 


\begin{abstract}
AUTORIZO A REPRODUÇÃO E DIVULGAÇÃO TOTAL OU PARCIAL DESTE TRABALHO, POR QUALQUER MEIO CONVENCIONAL OU ELETRÔNICO, PARA FINS DE ESTUDO E PESQUISA, DESDE QUE CITADA A FONTE.
\end{abstract}

Ficha catalográfica preparada pela Seção de Tratamento da Informação do Serviço de Biblioteca - EESC/USP

Leite, Luciano Gobo Saraiva

Desenvolvimento de modelos numéricos para análise de problemas de interação de domínios bidimensionais / Luciano Gobo Saraiva Leite ; orientador Wilson Sérgio Venturini. -- São Carlos, 2007.

Tese (Doutorado- Programa de Pós-Graduação em Engenharia de Estruturas. Área de Concentração: Métodos Numéricos) -- Escola de Engenharia de São Carlos da Universidade de São Paulo, 2007.

1. Método dos Elementos de Contorno. 2. Multidomínios. 3. Condensação de domínios. 4. Mecânica de dano. 5. Regeneração de domínios. I. Título. 


\section{FOLHA DE JULGAMENTO}

Candidato: Engenheiro LUCIANO GOBO SARAIVA LEITE

Tese defendida e julgada em-26/02/2007 perante a Comissão Julgadora:

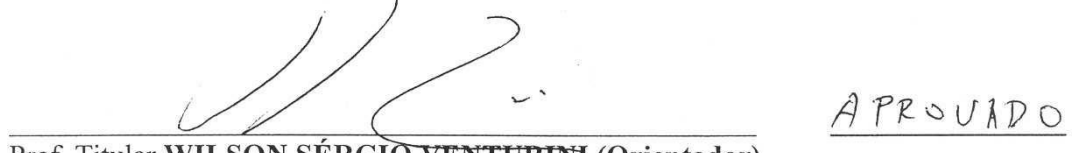

Prof. Titular WILSON SÉRGIO VENTURINI (Orientador)

(Escola de Engenharia de São Carlos/USP)

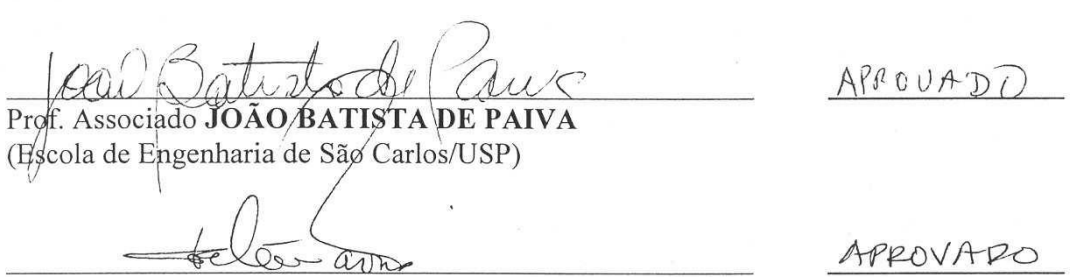

Prof. Titular WALTER SAVASSI

(Escola de Engenharia de São Carlos/USP)

LoS1

(Universidade Estadual de Campinas/UNICAMP)

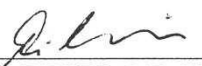

APRUVADO

Prof. Dr. GABRIEL DE OLIVEIRA RIBEIRO

(Universidade Federal de Minas Gerais/UFMG)

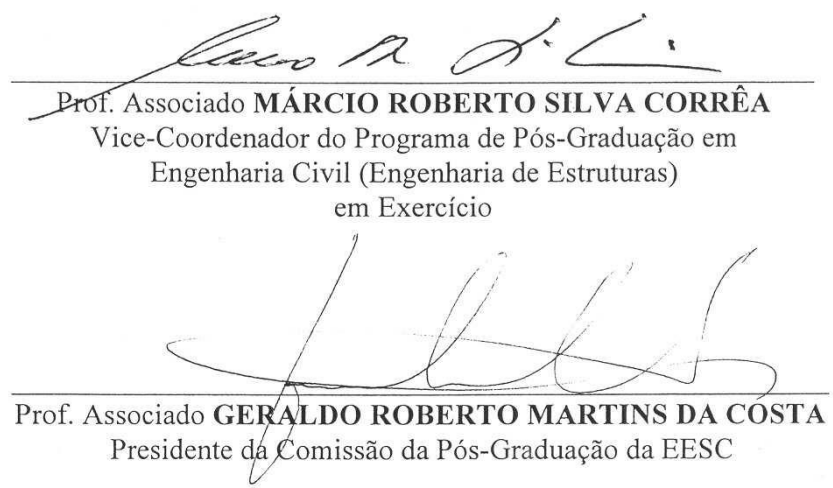



À minha esposa Sandra

e aos filhos Rômulo e

Dafne 


\section{Agradecimentos}

À Deus em primeiro lugar, por sua imensa bondade e por ter-me dado saúde e paz para prosseguir neste trabalho.

Aos meus pais, sogro e sogra, pelo apoio que tive e pela dádiva da vida. Em especial à minha mãe Dirce que me apoiou bastante e se orgulha muito deste trabalho.

À minha família (Sandra, Rômulo e Dafne) pelo tempo e a paciência roubados de vocês.

Ao povo brasileiro que, com o suor de seu trabalho, contribuiu com os impostos necessários para financiar este trabalho. Em retribuição lhe ofereço este trabalho para o seu desenvolvimento técnico e científico.

Ao $\mathrm{CNPq}$ pela ótima administração do dinheiro público que custeou minha bolsa de doutorado.

À USP, especialmente à EESC, pelas ótimas instalações e excelente corpo docente, os quais foram imprescindíveis para a realização desta tese.

Ao meu orientador Prof. Wilson Sergio Venturini, que foi mais que um orientador, foi um amigo e que, mais que ensinar, me mostrou como descobrir.

Aos professores do Departamento João Batista Paiva, Humberto Breves Coda e Márcio Roberto Silva Correa pela preciosa ajuda quando necessitei.

Aos professores da Escola de Engenharia da UFMG, Ney Amorim Silva, Gabriel Oliveira Ribeiro e Eduardo Chahud, que acreditaram inicialmente neste trabalho.

Aos funcionários do Departamento de Estruturas da EESC, Rosi Aparecida Jordão Rodrigues, Maria Nadir Minatel, Rodrigo Ribeiro Pacolla, Masaki Kawabata Neto e Antonio Valdir Carneiro pela enorme dedicação.

Aos amigos do departamento, Valério da Silva Almeida, Oscar Bayardo Ramos Lovon, Alexandre Sampaio Botta, Wilson Wesley Wutzow, Leandro Vanalli, Faustino Sanches Júnior, Angelo Vieira Mendonça e Josafá de Oliveira Filho pela amizade e pelo companheirismo nos momentos mais difíceis, ajudando a EESC a ser 
um ambiente mais aconchegante.

Ao Banco Nossa Caixa, empresa a qual tenho orgulho e honra de fazer parte, por sempre ter me apoiado e ajudado neste projeto quando necessário. Aos funcionários das Agências Corumbataí, Ibaté e Vila Prado pelo companheirismo e ombro amigo quando precisei. Ao Sr. José Natal Rebeschini, gerente de Corumbataí, que com sua inabalável fé e gerenosidade, foi o amigo certo nas horas incertas.

A todos aqueles que contribuíram com este trabalho, que não foram citados aqui, desculpem a falta de memória. 
LEITE, L.G.S. (2007) - Desenvolvimento de modelos numéricos para análise de problemas de interação de domínios bidimensionais. 2007. 160f. Tese (Doutorado) - Escola de Engenharia de São Carlos, Universidade de São Paulo, São Carlos 2007.

Neste trabalho foi desenvolvida uma formulação para análise de sólidos bidimensionais constituídos por multiregiões utilizando-se do Método dos Elementos de Contorno para análise linear e não linear. Para o caso de análise linear foi estudado o caso de regiões constituídas por sub-regiões de diferentes características mecânicas, utilizando-se técnicas que inicialmente consideram a compatibilidade de deslocamentos e o equilíbrio de forças na interface entre as sub-regiões, antes de se escrever as equações de equilíbrio. Inicialmente foi feita uma formulação, chamada neste trabalho de formulação singular, onde leva-se em conta apenas os deslocamentos incógnitos na interface e, posteriormente, foi desenvolvida outra formulação denominada hipersingular, onde são preservadas na interface apenas as forças de superfície. Para inclusões muito esbeltas, foi utilizada a técnica da condensação de domínios, onde o domínio 2D foi condensado inicialmente em um domínio linear de fibra e posteriormente em viga. Foi utilizada a discretização de inclusões muito esbeltas com rigidez quase nula visando a simular o comportamento de uma região de fratura elástica. A formulação foi estendida para análise não linear. A técnica das tensões iniciais foi adotada para modelar o sólido com regiões danificadas. Foi adotada a degeneração de inclusões muito esbeltas, que obedecem as leis constitutivas não lineares da mecânica do dano, simulando a origem de uma região de fratura. Para se melhorar a precisão das integrais, foi adotada a integração analítica sobre todo contorno e também sobre o domínio. Foram testados vários exemplos para validar os modelos propostos.

Palavras-chave: Método dos Elementos de Contorno, Multidomínios, Condensação de Domínios, Mecânica do Dano, Degeneração de domínios. 


\section{Abstract}

LEITE, L.G.S. (2007) - Development of numerical models for interaction problems of two-dimensional domain analysis. 2007. 160f. Thesis (Doctoral) - Escola de Engenharia de São Carlos, Universidade de São Paulo, São Carlos (2007).

In this work, a boundary element formulation was developed to analyze 2D multiregions solids formed in the context of linear and non-linear analysis. Linear analysis was adopted to study problems containing regions with different elastic parameters. This formulation was used to study inclusion that could be degenerated to thin inclusion to represent the behavior of fibers and beams embedded in the main solid. For the linear problems, the sub-regions were adopted to represent structural elements with different mechanical characteristics. The sub regions were joined together by assuming the classical hypotheses of displacement compatibility and traction equilibrium along the interfaces, but applied before the approximation of the boundary and interface values. The alternative sub-region technique was developed initially to eliminate traction values along the interfaces, introducing therefore only unknown displacements. The technique was then modified to eliminate all displacements along the interface preserving the traction as unknowns. For the case of very thin inclusions the formulation has been simplified to simulate fiber and beam reinforcements. Appropriate displacement approximations across the thin sub-region have been assumed. In this inclusion was also analyzed with the Elastic modulus degenerating to zero, simulating therefore a crack problem. The formulation has been extended to non-linear analysis. The initial stress procedure has been adopted to model solid with damaged regions. The damaged regions were assumed to be very small to simulate non-linear crack behavior governed by damage mechanic models. To improve the quality of the results all boundary and domains were integrated analytically. Many examples have been tested to certify that the proposed models are reliable.

Keywords: Boundary Element Method, Multi-domain, Domain Condensation, Damage, Domain Degeneration. 


\section{LISTA DE FIGURAS}

2.1 Força de contato atuando em um determinado plano de corte . . . . . 18

2.2 Tensões nas faces normais aos eixos cartesianos . . . . . . . . . . . 19

2.3 Variação das tensões no elemento infinitesimal . . . . . . . . . . . . . 20

2.4 Tensões atuantes nas faces do elemento de superfície definida pela normal n . . . . . . . . . . . . . . . . . . . . . . . . . 22

2.5 Relação entre o sistema ortogonal $x_{1}, x_{2}, x_{3}$ e o eixo $\bar{x}_{1} \ldots \ldots$. . . . . . 23

2.6 Deformações axiais e angulares . . . . . . . . . . . . . . 27

2.7 Deslocamento e rotação rígida sobre o plano $x_{1}-x_{2} \ldots \ldots$. . . . . . 29

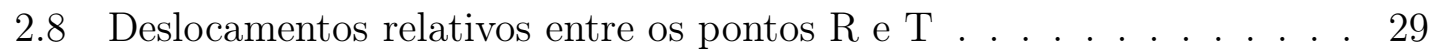

2.9 Exemplos de Estado Plano de Deformação . . . . . . . . . . . . . . 38

2.10 Exemplo de Estado Plano de Tensão . . . . . . . . . . . . . . . . . . 40

3.1 Força concentrata em região plana cilíndrica . . . . . . . . . . . . . 46

3.2 Função Delta de Dirac . . . . . . . . . . . . . . . . . . . . . . . . . . 49

3.3 Sólido de área $\Omega$ e contorno $\Gamma$ contido num sólido de dimensões infi-

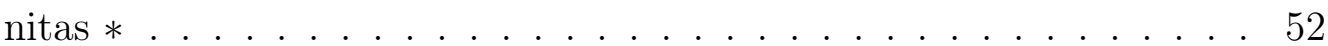

3.4 Contorno adicional com raio tendendo a zero . . . . . . . . . . . . 54

3.5 Geometria de um contorno de canto anguloso . . . . . . . . . . . 57

3.6 Discretização do contorno - elementos retos e aproximação linear de $U$ e $P \ldots \ldots \ldots \ldots \ldots \ldots$

3.7 Funções aproximadoras e coordenadas paramétricas . . . . . . . . . . 61

3.8 Possíveis posições do ponto fonte em integração analítica não singular 64

3.9 Coordenadas locais para integração analítica com ponto fonte não alinhado com o elemento . . . . . . . . . . . . . . . . . 64

3.10 Funções aproximadoras e variáveis locais para integração . . . . . . . 66 
3.11 Funções aproximadoras em elementos alinhados com o ponto fonte a) Ponto fonte atrás do elemento b) Ponto fonte à frente do elemento . . 67

3.12 Relação entre eixos globais e eixo local do elemento . . . . . . . . . . 67

3.13 Subelementação padronizada . . . . . . . . . . . . . . . . . . . 69

3.14 Subelementação progressiva . . . . . . . . . . . . . . . . 70

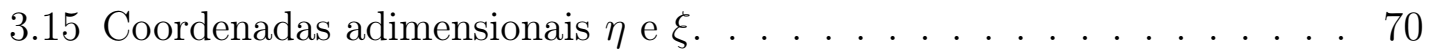

3.16 Funções de forma do elemento. . . . . . . . . . . . . . . . . . . 71

3.17 Determinação dos ângulos para o sub-elemento. . . . . . . . . . . . 71

3.18 Determinação do comprimento para o sub-elemento. . . . . . . . . . . 72

3.19 Comprimento do sub-elemento para $\Psi$ maior que $60^{\circ} \ldots$. . . . . . . 73

4.1 Região com multidomínios . . . . . . . . . . . . . 76

4.2 Deslocamentos através da espessura da fibra . . . . . . . . . . . . . 82

4.3 Múltiplo domínio com inclusão de barra . . . . . . . . . . . . . . . . 83

4.4 Deslocamentos através da espessura da viga . . . . . . . . . . . . . 84

4.5 Estaca em meio contínuo . . . . . . . . . . . . . . . . . . . 87

4.6 a) Discretização do meio contínuo b) Discretização da base da estaca

c) Discretização da base da estaca e posição dos nós da formulação de barra. . . . . . . . . . . . . . . . . . . . . . . . . 88

4.7 Deslocamento vertical ao longo do fuste devido à força $F_{v} \ldots \ldots$. . . 88

4.8 Deslocamento horizontal ao longo do fuste devido à força $F_{h}$. . . . . 89

4.9 Deslocamento horizontal ao longo do fuste devido ao momento $M$. . 89

4.10 Força tangencial à interface devida à força $F_{v} \ldots \ldots$. . . . . . . . 90

4.11 Força tangencial à interface devida à força $F_{v}$, apenas MEC . . . . . 90

4.12 Força normal à interface devida à força $F_{h}$. . . . . . . . . . . . . . 91

4.13 Força normal à interface devida à força $F_{h}$, apenas MEC . . . . . . . 91

4.14 Força normal à interface devida ao momento $M$. . . . . . . . . . . . 92

4.15 Força normal à interface devida ao momento $M$, apenas MEC . . . . 92

4.16 Sólido com inclusão. Características geométricas e discretização . . 93

4.17 Tensão normal atuante através da seção A-B . . . . . . . . . . . . . . 94

4.18 Tensão cisalhante atuante através da seção A-B . . . . . . . . . . . . 95

4.19 Sólido com fratura . . . . . . . . . . . . . . . . . . 96 
4.20 Tensão $\sigma_{x}$ após a ponta da fratura para $\alpha=0^{\circ} \ldots$. . . . . . . . 97

4.21 Tensão $\sigma_{x}$ e $\tau_{x y}$ após a ponta da fratura para $\alpha=30^{\circ} \ldots \ldots 7$

4.22 Domínio quadrado com inclusão e condições de contorno . . . . . . . 98

4.23 Deslocamento vertical ao longo do eixo $x$ com diferentes módulos de rigidez longitudinal da inclusão . . . . . . . . . . . . . . . . . . . . 99

$4.24 \sigma_{y} / p$ ao longo da abscissa $x \ldots \ldots$. . . . . . . . . . . . 99

4.25 Tensão $\sigma_{x}$ ao longo do domínio principal e da inclusão . . . . . . . . . 100

4.26 Domínio com inclusão de fibras. Dimensões e discretização. . . . . . . . 101

4.27 Tensão normal nas proximidades da fibra. . . . . . . . . . . . . . 102

4.28 Deslocamentos para vários valores de $w_{f} / w_{b} \ldots$. . . . . . . . . . . 102

4.29 Força tangencial na fibra inferior para vários valores de $w_{f} / w_{b} \ldots$. . 103

4.30 Deslocamento longitudinal com $\alpha=1.0$ e $w_{f} / w_{b}=005 \ldots$. . . . . 103

4.31 Força tangencial na interface com $\alpha=1.0$ e $w_{f} / w_{b}=005 \ldots$. . . . . 104

4.32 Força tangencial na interface com várias rigidezes . . . . . . . . . . . 104

5.1 Campo das tensões iniciais . . . . . . . . . . . . . . . . . . . 108

5.2 Discretização do domínio em células triangulares . . . . . . . . . . . . 111

5.3 Coordenadas cilíndricas $r$ e $\theta$. . . . . . . . . . . . . . 112

5.4 Relação entre o eixo global e o eixo local da aresta da célula . . . . . 113

5.5 Nós internos e externos ao contorno da célula . . . . . . . . . . . . . 114

5.6 Elemento de domínio com aproximação constante . . . . . . . . . . . 117

5.7 Relação tensão x deformação - Modelo de Dano . . . . . . . . . . . . 121

5.8 Funções $f_{t}$ e $f_{c}$ definidas no espaço de tensões (Haigh-Westergaard). Figura retirada de Comi $(2001)$. . . . . . . . . . . . . . 131

5.9 Orientação dos eixos locais da sub-região de origem da fratura . . . . 136

5.10 Deslocamentos relativos entre a espessura da fratura . . . . . . . . . 137

5.11 Características geométricas e discretização .......... . 142

5.12 Dano correspondente a 6 incrementos de carga . . . . . . . . . . . . . 142

5.13 Dano na tração ' $D_{t}$ 'correspondente a 7 incrementos de carga . . . . . 143

5.14 Sólido com sub-região de origem de fratura . . . . . . . . . . . . . . . 144

5.15 Tensão $\sigma_{\bar{x}} \mathrm{X} \Delta \bar{u}$ para $\mathrm{h}=2 \times 10^{-4} \ldots \ldots$. . . . . . . . . . 145

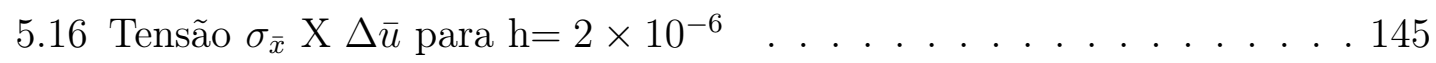


5.17 Abertura $\Delta \bar{u}$ ao longo da fratura para $\mathrm{h}=2 \times 10^{-4} \ldots 146$

5.18 Abertura da fratura em um modelo discreto . . . . . . . . . . . . . 146 


\section{CONTEÚDO}

1. Introdução . . . . . . . . . . . . . . . . . . . . 1

1.1 Considerações Iniciais . . . . . . . . . . . . . . . . . . . . 1

1.2 Revisão Bibliográfica . . . . . . . . . . . . . . . . . . . . . 9

1.2.1 Estruturas com multidomínios, técnica de sub-domínios 10

1.2 .2 Inclusões . . . . . . . . . . . . . . . . . . . . . 10

1.2.3 Acoplamento MEC/MEF . . . . . . . . . . . . 11

1.2.4 Técnica de condensação de domínio . . . . . . . . . . . . 11

1.2.5 Interação Solo-Estrutura . . . . . . . . . . . . . . . . . . 12

1.2.6 Análise Não-Linear . . . . . . . . . . . . . . . . . . . . . . 12

1.2.7 Mecânica da Fratura Elástica Linear utilizando o MEC 13

1.2.8 Mecânica da Fratura Não-Linear . . . . . . . . . . . . . . 14

1.2.9 Mecânica do Dano Contínuo . . . . . . . . . . . . . . 15

1.2.10 Evolução da Fratura através de Processo de Danificação 16

2. Teoria da Elasticidade . . . . . . . . . . . . . . . . . . . 17

2.1 Introdução . . . . . . . . . . . . . . . . . . . . . . . . 17

2.2 Leis constitutivas elásticas . . . . . . . . . . . . . . . . . . 17

2.2 .1 Tensão . . . . . . . . . . . . . . . . . 17

2.2 .2 Deformação . . . . . . . . . . . . . . . 27

2.2.3 Pequenas deformações e rotações em duas dimensões . 27

2.2.4 Deslocamentos relativos em 2D . . . . . . . . . . 29

2.2.5 Deformações principais e mudança de coordenadas . . 32

2.2.6 Equações Constitutivas . . . . . . . . . . . . . . . 33

2.2.7 Lei de Hooke generalizada - Análise Isotrópica . . . . . 34

2.2.8 Equação de Navier . . . . . . . . . . . . . . . . 36 
2.2.9 Elasticidade Plana - 2D . . . . . . . . . . . . 37

2.2.10 Estado Plano de Deformação . . . . . . . . . . . . . . 37

2.2.11 Estado Plano de Tensão . . . . . . . . . . . . . . . . . 40

3. Método dos Elementos de Contorno . . . . . . . . . . . . . . . . . . 43

3.1 Introdução . . . . . . . . . . . . . . . . . . . . . . . . 43

3.2 Vetor de Galerkin . . . . . . . . . . . . . . . . . . . . 43

3.3 Problemas de Kelvin em Elasticidade Plana . . . . . . . . . . . 45

3.3.1 Equação fundamental de deslocamento . . . . . . . . . . 48

3.3.2 Equação Fundamental de Tensão . . . . . . . . . . . . . . 50

3.3.3 Equação Fundamental de Forças de Superfície . . . . . 51

3.4 Formulação Direta de Elemento de Contorno . . . . . . . . . . 52

3.5 Formulação algébrica das equações de contorno . . . . . . . . . 60

3.6 Integrais Exata . . . . . . . . . . . . . . . . . 63

3.7 Técnicas de Subelementação . . . . . . . . . . . . . . . . . . 69

4. Análise de Regiões com Multidomínios via MEC . . . . . . . . . . . 75

4.1 Introdução . . . . . . . . . . . . . . . . . . . . . . 75

4.2 Sub-regiões . . . . . . . . . . . . . . . 76

4.2.1 Formulação Singular . . . . . . . . . . . . . . 76

4.2.2 Formulação Hiper-singular . . . . . . . . . . . . . . . 79

4.3 Técnicas de Condensação de Domínios . . . . . . . . . . . . . . 81

4.3.1 Transformação de Domínios 2D em Fibras . . . . . . . . 81

4.3.2 Transformação de Domínios 2D em Vigas . . . . . . . . 83

4.4 Análise numérica . . . . . . . . . . . . . . . . . . . 86

4.4.1 Estaca em Meio Contínuo . . . . . . . . . . . . . . 86

4.4.2 Viga de concreto armado . . . . . . . . . . . . . . 93

4.4.3 Fratura Elástica - Sólido com fraturamento . . . . . . . 96

4.4.4 Domínio com inclusão de rigidez infinita . . . . . . . . . 98

4.4.5 Domínio enrijecido por fibras . . . . . . . . . . . . 101

5. Análise não-linear . . . . . . . . . . . . . . . . . . . . . . . 107

5.1 Introdução . . . . . . . . . . . . . . . . . . . . . 107 
5.2 Campo das tensões iniciais . . . . . . . . . . . . . . 107

5.2.1 Discretização das Integrais de Domínio - Elementos com aproximação linear . . . . . . . . . . . . . 111

5.2.2 Integração em linha das integrais de domínio . . . . . . 115

5.2.3 Discretização das Integrais de Domínio - Elementos com aproximação constante . . . . . . . . . . . . 116

5.3 Análise não-linear via Método dos Elementos de Contorno . 117

5.4 Mecânica do Dano . . . . . . . . . . . . . . . . . . . . . . . . . . . 120

5.4.1 Dano Simples . . . . . . . . . . . . . . . . . 121

5.4.2 Processo não-linear incremental - Dano Simples . . . . 124

5.4 .3 Modelo de Dano do Concreto . . . . . . . . . . . . . . 129

5.4.4 Processo não-linear incremental - Dano do Concreto . 132

5.5 Localização do Dano dando início à Fratura . . . . . . . . . . . 136

5.5.1 Processo não linear incremental . . . . . . . . . . . . 139

5.6 Análise Numérica . . . . . . . . . . . . . . . . . . . . . 141

5.6.1 Modelo de Dano escalar . . . . . . . . . . . . . . 141

5.6.2 Modelo de Dano do concreto . . . . . . . . . . . . . . 143

5.6.3 Degeneração de dano simples originando fratura . . . . 144

6. Considerações Finais . . . . . . . . . . . . . . . . . . . . . 149

Referências Bibliográficas . . . . . . . . . . . . 153 


\section{INTRODUÇÃO}

\subsection{Considerações Iniciais}

Com o advento dos computadores de grande capacidade de processamento, surgiram vários métodos numéricos para análise de problemas de engenharia, tais como na área da mecânica dos sólidos e na engenharia das estruturas. Um dos primeiros métodos surgidos foi o Método das Diferenças Finitas, seguido pelo Método dos Elementos Finitos e depois pelo Método dos Elementos de Contorno. Ultimamente, no caso da mecânica dos sólidos, apenas os dois últimos métodos têm sido mais frequentemente utilizados, assim como suas variações.

O método das diferenças finitas consiste da discretização do meio físico a ser analisado, utilizando-se nós sobre o cruzamento de linhas que, usualmente, são paralelas ao sistema cartesiano. A equação diferencial governante do problema físico é escrita para os nós através de uma aproximação em termos de diferenças finitas entre os nós para gerar um sistema algébrico de equações. Uma das vantagens do método é que seu uso é amplamente geral, sendo utilizado principalmente na mecânica dos fluídos, pois o meio fluído possibilita uma discretização em malha bastante regular, já que o domínio a ser analisado nesta área possui contornos com geometria sem grandes angulosidades e as condições de contorno são bastante simples. Como desvantagem o método exige uma malha muito fina e regular, o que o torna bastante limitado para problemas da mecânica dos sólidos, pois estes podem ter condições de contorno variáveis e superfícies bastante complexas, o que inviabiliza a utilização de uma malha regular.

O método dos elementos finitos consiste na divisão do corpo em elementos, chamados de elementos finitos, onde a equação governante do problema físico é transformada numa equação para cada elemento. Tais equações são integradas sobre o elemento, com a ajuda de funções aproximadoras, usualmente funções polinomiais, 
que interpolam o resultado desta integração aos nós do elemento. Com a representação da equação governante aproximada para os nós e da condição de contorno do problema representado em cada nó, monta-se um sistema linear algébrico, que pode ser resolvido por qualquer técnica de resolução de sistemas lineares.

O MEF é o método numérico mais utilizado devido principalmente a sua robustez, simplicidade de sua formulação e pelo fato de produzir uma matriz de rigidez simétrica e em banda que, aliada às técnicas especiais de resolução destas matrizes, reduz muito o tempo de processamento, principalmente em estruturas mais complexas. Por ser um método de domínio, isto é, as equações de equilíbrio e compatibilidade de deslocamento devem ser escritas para todos os pontos da estrutura, possui a desvantagem para análise de casos de sólidos com domínios considerados infinitos, pois todo o sólido precisa ser discretizado em inúmeros elementos sendo que, o que interessa na análise é o resultado em apenas algumas regiões. Outra desvantagem do método diz respeito a regiões de concentração de tensão, pois os deslocamentos e forças são aproximados nos nós e quando esses valores tendem ao infinito o método perde a precisão.

O método dos elementos de contorno é um método de fronteira, isto é, as incógnitas do problema, ou seja, deslocamentos ou forças de superfície, estão no contorno da estrutura, diminuindo a quantidade de incógnitas do problema. A formulação consiste em equações integrais de deslocamentos e de forças de superfície para o sólido, onde o domínio de integração é transformado para o contorno da estrutura, o qual é discretizado em elementos onde os valores são aproximados por funções aproximadoras e valores nodais. Agrupando-se todas as equações dos nós externos e impondo as condições de vinculação na estrutura, têm-se as incógnitas no contorno em termos de deslocamentos e de forças de superfície. Nos pontos internos os valores não fazem parte das incógnitas do problema e podem ser obtidos de maneira "exata", utilizando apenas os valores do contorno já obtidos.

O método tem como vantagem a diminuição de incógnitas do problema, principalmente no caso de domínios infinitos, pois pode-se discretizar parte do contorno do sólido e obter respostas apenas nos pontos desejados. Uma outra vantagem do método diz respeito a regiões de concentração de tensão, onde o método apresenta resultados bastante precisos, principalmente onde a variação dos resultados é muito 
brusca em uma pequena extensão. Como desvantagem o método apresenta matrizes de rigidez cheias, o que acarreta maior fluxo de processamento de análise e também possui problemas de integrais singulares, o que faz com que seja necessária a utilização de técnicas especiais de integração.

Um dos problemas usuais no estudo da mecânica dos sólidos é a consideração de inclusões, que são corpos inseridos em outros corpos. A geometria das inclusões pode ser qualquer, entretanto, merece especial destaque o estudo de inclusões onde a espessura é muito menor que suas outras dimensões, como por exemplo, as barras de aço em uma viga de concreto armado, pois tais inclusões apresentam problemas de modelagem numérica devido a proximidade das faces entre a espessura. As características mecânicas das inclusões variam de muito rígidas a extremamente flexíveis.

No caso de inclusão mais rígida do que o domínio que as contém encontram-se, por exemplo, os domínios inseridos em um meio contínuo, característica usualmente vista em problemas da construção civil. Como exemplo desse tipo de inclusão temse o caso de estacas, blocos, sapatas e tubulões, que estão inseridos em um meio menos rígido, que é o solo. Há inclusões naturais nos maciços; podem ser rígidas ou muito deformáveis, isotrópicas ou anisotrópicas. Uma outra técnica, utilizada principalmente para melhorar as características mecânicas de certas peças estruturais, consiste em se inserir fibras ou barras extremamente finas em um sólido contínuo corrigindo certas deficiências deste sólido. Tem-se no concreto armado um dos exemplos de inclusões mais rígidas que o meio contínuo que o cerca e neste caso suprimindo a deficiência da resistência à tração do concreto.

Para o caso de inclusões com rigidez baixa ou mesmo nula, cita-se como exemplo o caso de dutos (eletrodutos, encanamentos de água) que podem modificar significativamente o estado de tensão do sólido no qual estão inseridos. Um outro tipo de exemplo é o caso do aparecimento de microfissuras e fissuras no sólido, onde essas fissuras se comportam como uma inclusão de rigidez nula no corpo principal.

Uma das técnicas numéricas mais utilizadas para se analisar sólidos com inclusões ou contendo regiões com diferentes características mecânicas é a da sub-região. Tal técnica consiste em se discretizar as diferentes regiões do sólido com suas características mecânicas, sendo que para cada subdomínio são escritas equações de 
forças de superfície e de deslocamentos para os nós do contorno e da interface, o que leva a ter vários sistemas algébricos locais. Impondo o equilíbrio de forças e a compatibilidade de deslocamentos nos nós de interface, reunem-se os vários sistemas de equações locais em um sistema de equilíbrio global, gerando uma matriz esparsa, pois os únicos nós pertencentes às duas sub-regiões são os nós da interface.

Uma outra técnica de análise numérica, de regiões com inclusões, consiste no acoplamento do MEC com o MEF. Discretiza-se o domínio principal com as equações do MEC onde somente as variáveis do contorno da estrutura e os nós de acoplamento com os nós de elemento finito das inclusões serão considerados, e as inclusões são discretizadas segundo o MEF em elementos de barra, de casca ou sólido. Tal procedimento possui respostas bastante satisfatórias em termos de deslocamentos, mas para o caso das forças de contato, há fortes oscilações causadas pela tendência dos nós de reestabelecer o equilíbrio estático do elemento. Os resultados em si não se apresentam corretos, entretanto, se apresentam corretos na média.

Em 1992 Venturini (Venturini, 1992), propôs uma alternativa para a técnica das sub-regiões, onde o equilíbrio de forças de superfície é imposto antes da descretização, levando a um sistema de equações que não apresentam incógnitas na interface em termos de forças de superfície. Esse procedimento levou o nome de formulação singular devido ao tipo de singularidade envolvida nas integrais utilizadas na interface.

Tal técnica consiste em se somar as equações de deslocamentos das duas subregiões adjacentes ao nó de interface, levando-se em conta a compatibilidade de deslocamentos e o equilíbrio de forças de superfície nesta região. Para que as variáveis de forças de superfície da interface desapareçam do sistema de equilíbrio, deve-se multiplicar os termos das equações de deslocamentos de cada sub-região, pelo correspondente módulo de rigidez transversal que aparece no denominador da solução fundamental de deslocamentos, a qual irá formar a matriz de influência que multiplicará as incógnitas de forças de superfície. Após esta soma permanecem como incógnitas nos nós da interface apenas deslocamentos. O termo livre de deslocamento na interface é o mesmo dos nós de contorno.

Uma formulação análoga pode ser obtida preservando-se as forças de superfície. Nessa formulação ocorre o contrário, somam-se as equações de forças de superfície 
de ambos os lados da interface, levando-se em conta o equilíbrio estático e a compatibilidade de deslocamentos. Para se eliminar as variáveis de deslocamentos da interface, deve-se dividir as equações de forças de superfície dos sub-domínios adjacentes pelo correspondente módulo de rigidez transversal, pois tal termo se apresenta no numerador das soluções fundamentais contidas na matriz de influência de tensão, que multiplica as variáveis de deslocamentos. Tal formulação foi denominada de hipersingular, devido as fortes singularidades envolvidas nas equações de tensão utilizadas na interface. Em ambas as formulações, para que a soma se concretize, o coeficiente de Poisson das sub-regiões adjacentes a interface devem ser iguais.

Para se encontrar a tensão nos pontos internos, escrevem-se as equações de tensão do MEC e, nos pontos de interface, adotam-se os procedimentos da formulação singular ou hipersingular. Essa técnica diminui as incógnitas do problema e também contribui para que a matriz algébrica do problema se apresente mais compacta e cheia.

Utilizando-se o MEC para análise de inclusões muito finas, pode-se chegar a um sistema de equações matriciais com certa dependência linear, pois na medida em que a inclusão se torna mais fina, as distâncias entre os pontos fontes das interfaces opostas diminuem, chegando a um ponto em que a equação de um ponto passa a ser quase ou senão idêntica à equação do ponto oposto da inclusão. Para contornar esse problema foi adotada uma condensação de equações de modo que o domínio 2D fosse transformado em um domínio 1D.

Numa primeira etapa considerou-se o campo de deslocamento constante ao longo da espessura da inclusão. Nesse caso as incógnitas são representadas somente nos nós centrais das inclusões através da simples soma dos deslocamentos dos nós das faces opostas da inclusão. A inclusão então passa a ter o comportamento mecânico de fibra, pois, como os deslocamentos são constantes, não há rotação associada aos nós.

Numa segunda etapa considerou-se o deslocamento variando linearmente ao longo da espessura da inclusão, gerando uma formulação de barras fletidas. Nesse caso é necessária a criação de equações adicionais de rotação para nós no centro da inclusão. Nas duas hipóteses as respostas foram satisfatórias com fibras e barras muito finas. 
Um outro procedimento adotado nesse trabalho para se alcançar resultados mais precisos, foi o de se utilizar integração analítica sobre as equações envolvidas no modelo físico. Normalmente tal procedimento é utilizado para o caso de integração do ponto fonte no elemento ao qual ele pertence, por causa de problemas de singularidade. Nesse trabalho foi utilizada a integração analítica para todos os casos, inclusive para integrais de domínio, que serão utilizadas para análise não linear.

Nas integrais de contorno, para valores de deslocamentos e forças de superfície, as integrais foram feitas para um eixo local, com sentido seguindo a orientação do elemento a ser integrado, e depois rotacionadas para o eixo global. No caso das integrais de domínio, tensões e deformações, por causa da complexidade das funções de forma e da geometria dos elementos de célula, a integração analítica foi feita no eixo global, merecendo atenção especial as singularidades que ocorrem nas equações fundamentais de tensão e o termo livre das mesmas.

Com tal procedimento, os resultados obtidos foram mais precisos, principalmente no caso de domínios muito esbeltos e também no caso onde o ponto fonte está muito próximo ao elemento integrado, evitando-se até a utilização da técnica de subelementação.

Também foi observada a diminuição do tempo de processamento de cada análise, pois, com integrais numéricas para se obter uma boa precisão, é necessário utilizar muitos pontos de integração, se constituindo em uma vantagem competitiva em análise de estruturas mais complexas.

Alguns materiais utilizados na Engenharia Civil, como o concreto, embora possuam estrutura microscópica não homogênea, se comportam em escala macroscópica segundo as hipóteses da mecânica do contínuo, isto é, o material se comporta como um corpo homogêneo com características mecânicas iguais em todos os pontos da estrutura.

Os metais geralmente se apresentam macroscopicamente homogêneos sendo que, apenas nas dimensões atômicas, é que se apresenta certa heterogeneidade. Por isso, o processo de deformação se dá pelo escorregamento entre os planos dos cristais, gerando o efeito de plastificação e de viscoplastificação e, com o aumento dessas deformações, geram-se superfícies de escorregamento entre os cristais do metal. Esses escorregamentos geram pequenas microfissuras, que podem se propagar e aumentar 
de tamanho, gerando fraturas que possuem uma alta concentração de tensão na sua extremidade, para que a estrutura possa preservar sua estabilidade. À medida que, tal fratura avança pela plastificação progressiva da região em frente da fratura, a estrutura pode ser levada ao colapso.

No caso do concreto, o material já é, de certa forma, macroscopicamente heterogêneo, pois possui na sua constituição elementos de diferentes formas e tamanhos, como cimento, areia e brita, e também de diferentes resistências mecânicas. Com o aumento das deformações, formam-se pequenas microfissuras entre as interfaces dos diferentes constituintes do material, que progressivamente se ligam e formam macrofissuras que se unem, dando origem a superfícies de descontinuidades ou fraturas.

Neste trabalho as fraturas foram tratadas como sub-regiões extremamente finas, que possuem resistência mecânica nula, e aparecem em um sólido originando descontinuidades. É de suma importância o estudo do aparecimento e do desenvolvimento de fraturas em um sólido, para se conhecer até que ponto as fraturas comprometem a segurança da estrutura.

Inicialmente a fratura foi estudada considerando-se todo o sólido como tendo comportamento puramente elástico, negligenciava-se a plastificação ou deterioração do material nas extremidades da fratura, dando origem a mecânica da fratura linear. Tal formulação (Griffith, 1921) é baseada no equilíbrio energético para a formação da fratura e que, durante este evento, o sólido permanece com comportamento puramente elástico.

Como as hipóteses de Griffith, levam em conta as características globais da estrutura e, por ser a fratura um fenômeno que ocorre localmente, outros parâmetros foram incorporados tais como, a intensidade de tensão na vizinhança das extremidades da fratura, a sua orientação e o tipo de abertura. Com esses parâmetros (Irwin, 1957), foram desenvolvidos fatores de intensidade de tensão que dependem do tipo de abertura da fratura, da geometria do sólido e da posição da fratura em relação ao sólido, sendo possível assim conhecer teoricamente as tensões atuantes nas regiões próximas às extremidades da fratura, e sua influência sobre o comportamento do sólido.

A análise numérica mais comum de um sólido contendo fratura, consiste em se discretizar a fratura apenas com uma dimensão, no caso bidimensional, sendo 
que os deslocamentos e tensões nas extremidades da fratura são comparados com os fatores de intensidade de tensão.

Nesse trabalho foi adotada a análise de fratura com a técnica de sub-região, bastando para isso discretizar uma sub-região extremamente fina e adotar para a rigidez desse material um valor muito próximo de zero, aproximando o comportamento dessa sub-região ao comportamento de uma fratura. Com a utilização de integrais analíticas, elimina-se a aproximação das integrais numéricas, e pode-se chegar a subdomínios bastante esbeltos, com resultados bastante próximos dos obtidos pelas formulações teóricas da mecânica da fratura.

Enquanto que, a mecânica da fratura lida com a propagação de uma descontinuidade macroscópica em um meio contínuo, a mecânica do dano se preocupa com efeitos causados por microfissurações que podem ocorrer em uma área distribuída, as quais tendem a se unirem e se tornarem uma fissura discreta, podendo evoluir para uma fratura. Tal fenômeno pode ser descrito macroscopicamente no material, como a perda progressiva das propriedades mecânicas das regiões afetadas, como por exemplo, o módulo de elasticidade, até se chegar a constantes elásticas nessas regiões nulas onde se formará uma descontinuidade no sólido.

Em estruturas granulares, como o concreto, o dano tem papel preponderante na resposta não-linear do material, sendo que a mecânica do dano pode ser utilizada para construir modelos constitutivos bastante realistas para estas estruturas. Em síntese, na mecânica do dano, a resistência de uma estrutura é afetada pelo aparecimento e progressão de micro defeitos (micro fissuras ou poros), considerados continuamente distribuídos, enquanto que na mecânica da fratura, a resistência da estrutura é afetada pela evolução de um único defeito de forma pontiaguda, com o restante da estrutura intacta.

A formulação mais simples de dano é a do dano escalar, onde se obtém um módulo de elasticidade equivalente em função da evolução do dano $E_{e q}=(1-D) E$. Nas regiões do sólido onde o material se apresente íntegro o dano ' $D$ ' é zero e conforme o dano evolui o valor de ' $D$ ' pode chegar a 1 , sendo que nesta fase o material se encontra completamente deteriorado. Tal formulação se chama escalar porque um único parâmetro penaliza todas as constantes elásticas. Existem modelos mais complexos como o de (Mazars ; Pijaudier-Cabot, 1995), onde a danificação leva 
em conta fatores como a resistência do concreto à tração e à compressão bem como outros parâmetros, sendo também escalares.

Todos os modelos da mecânica do dano envolvem análise não-linear. A análise deve ser feita por incrementos de ações, que podem ser forças ou deslocamentos, os quais produzem resultados elásticos no sólido em termos de tensões e deformações, que são corrigidos segundo as leis constitutivas do material.

No método dos elementos de contorno a análise não-linear pode ser feita utilizan-do-se tensões iniciais no domínio do sólido. Com isso, novas integrais, agora de domínio, de tensão e deformação são introduzidas no sistema de equilíbrio do corpo. Esse procedimento é interessante, pois permite analisar vários tipos de problemas não-lineares.

No presente trabalho a integração do domínio foi feita discretizado-o em células triangulares com função aproximadora linear. As correções são feitas com incrementos de deformação para manter o equilíbrio do sólido, o qual é escrito com equações de deslocamentos do contorno e de tensão nos nós do domínio. Utilizouse o processo não linear de Newton-Raphson, onde as correções dos incrementos elásticos são feitas por matriz tangente.

Nesse trabalho estuda-se também o comportamento e a evolução de fraturas em concreto a partir da evolução inicial do dano, onde será utilizado o modelo de dano escalar, para se avaliar a evolução da danificação. Com o auxílio das integrais analíticas, pretende-se discretizar regiões muito esbeltas com aproximação constante das tensões iniciais a serem aplicadas. As variáveis de dano serão analisadas na direção da espessura da fratura, onde a variação da tensão se relacionará com a variação de deslocamentos. A resistência mecânica dessas sub-regiões será nula quando as variáveis de dano tiverem valor unitário, quando então se apresentará um comportamento de fratura.

\subsection{Revisão Bibliográfica}

O histórico que será abordado neste ítem se resume ao campo da interação entre regiões com características elásticas diferentes, tais como: inclusões, técnicas de condensação de domínios, acoplamento MEC/MEF, integração analítica, aná- 
lise não-linear utilizando o MEC, Mecânica da Fratura e Mecânica do Dano via MEC. Nesta seção as referências serão divididas por temas para que se facilite o entendimento do leitor.

\subsubsection{Estruturas com multidomínios, técnica de sub-domínios}

Nas estruturas de engenharia civil, muitas são compostas de materiais com características mecânicas diferentes, estão acopladas e trabalham em conjunto. Para se fazer uma análise numérica de tais estruturas, deve ser dada uma atenção especial na interface entre os materiais, compatibilizando os deslocamentos e o equilíbrio de forças ao longo do contato.

Uma técnica utilizada para análise de tais estruturas é a técnica de sub-domínios, onde o sólido é dividido em vários sub-domínios com característica físicas e mecânicas distintas. Um procedimento clássico para tal análise, utilizando o Método dos Elementos de Contorno é o de discretizar as sub-regiões separadamente e nas regiões de interface fazer a compatibilidade de deslocamentos e equilíbrio de forças, que serão consideradas no sistema global de equilíbrio da estrutura. Pode ser citado o trabalho de (Brebbia ; Dominguez, 1992) nesta área. Tal técnica possui a característica de gerar matrizes esparsas, requerendo técnicas especiais de resolução. Na EESC pode ser citado o trabalho de (Wutzow, 2003).

\subsubsection{Inclusões}

Um outro problema de regiões com multidomínios é o de sólido com inclusões, que são regiões contidas em uma outra região, podendo ser extremamente finas ou não. Podem ter rigidez maior que a do sólido e serem finas, e aí são chamadas de enrijecedores, pois conferem à estrutura um aumento da rigidez. Ou podem ter uma rigidez mínima que, dependendo de sua espessura, ter o comportamento de uma linha de fratura. Trabalhos com enrijecedores, principalmente dando rigidez às placas, foram feitos na EESC por (Wutzow, 2003), (Fernandes, 2003), (Carmo, 2003) e (Agostinho, 1998).

Uma das principais técnicas utilizadas para se discretizar as inclusões muito finas, no caso de elasticidade bidimensional, é a de discretizá-las com elementos 
lineares, evitando que se discretize as faces opostas na direção da espessura da inclusão, evitando-se assim problemas de dependência linear no sistema algébrico de equilíbrio. As técnicas de Elementos de Contorno mais utilizadas para essa análise são o acoplamento MEC/MEF e a condensação de domínios 2D em domínios 1D.

\subsubsection{Acoplamento MEC/MEF}

No acoplamento do Método dos Elementos de Contorno (MEC) com o Método dos Elementos Finitos (MEF), discretizam-se os domínios com dimensões maiores em elementos de contorno, onde a técnica do MEC necessita de menos incógnitas, pois utiliza apenas as variáveis do contorno, e as inclusões em elementos finitos de fibra ou de viga. Nesse campo citam-se os trabalhos de (Coda ; Venturini, 1999) e (Botta ; Venturini, 2005) com acoplamento MEC/MEF e também na EESC podem ser citados os trabalhos de (Ribeiro, 2005), (Oshima, 2004), (Almeida, 2003), no campo da interação solo/estrutura e por (Paccola, 2004), (Vanalli, 2004) na análise não linear dos sólidos.

\subsubsection{Técnica de condensação de domínio}

A outra técnica diz respeito à análise de regiões com inclusões muito finas, utilizando-se o MEC para discretizar o domínio principal e as inclusões. O domínio das inclusões é degenerado em elementos lineares com características de fibra, considerando como constantes os deslocamentos entre as faces opostas da inclusão, portanto, considera-se nula a rotação. No caso de vigas, considera-se que a variação dos deslocamentos entre as faces opostas da inclusão é linear, podendo-se assim considerar a rotação relativa dos nós.

Podem ser citados os trabalhos de Banerjee (Banerjee, 1981), onde domínios 3D, discretizados com elementos de célula e onde as inclusões, neste caso cilíndricas, foram degeneradas em elementos lineares, e os trabalhos de (Leite; Venturini ; Coda, 2003) e (Leite; Venturini ; Coda, 2005) onde as inclusões foram primeiramente degeneradas em fibras e posteriormente em vigas. 


\subsubsection{Interação Solo-Estrutura}

Uma aplicação para o caso de domínio com inclusões é o da interação soloestrutura. Nesse caso pode-se discretizar o solo em elementos de contorno, pois é um domínio que tende ao infinito e estacas ou sapatas com elementos finitos ou mesmo elementos de contorno. Nesse campo cita-se o trabalho de (Almeida, 2003), onde foi utilizada uma análise 3D do problema e, tanto o solo quanto as estruturas de fundação foram discretizadas em elementos de área do MEC, analisando inclusive solo com várias camadas de diferentes características elásticas.

Outro tipo de análise foi feita por Leite, (Leite, 1998) onde foi acoplado, em uma placa formulada via MEC utilizando a Teoria de Reissner (Silva, 1996), elementos de viga do MEF. Os elementos de viga foram utilizados para discretizar estacas ou tubulões sendo que, nesses últimos, foram utilizados elementos com características de rigidez diferentes para se simular o alargamento da base. A resistência lateral do solo foi considerada como carga distribuída, aplicada nos nós dos elementos segundo fatores desenvolvidos por (Bowles, 1988), que variam com a profundidade do solo. A técnica consiste em primeiro se encontrar a resistência da estaca em um determinado tipo de solo e passar esta resistência, através de coeficientes de molas elásticas de deslocamentos lineares e de rotações para a placa, onde esta, ao ser carregada, se apóia em 'molas' elásticas simulando a resistência elástica do solo e em molas advindas da resistência da estaca, determinadas por uma análise via MEF.

Na EESC citam-se os trabalhos de (Ribeiro, 2005), (Oshima, 2004), (Almeida, 2003) e (Mendonça, 1997).

\subsubsection{Análise Não-Linear}

Uma das áreas mais pesquisadas em análise numérica ultimamente é o comportamento não linear de estruturas, pois visam simular seu real comportamento. Pode-se citar os principais tipos de comportamento não linear das estruturas tais como a plastificação nos metais, o dano em estruturas granulares e a fratura nãolinear.

O processo tradicional de análise numérica de tais comportamentos é o da matriz tangente (Owen ; Hinton, 1980), Newton-Raphson. O processo não linear aqui 
implantado permite apenas incrementos de forças de superfície e deslocamentos, porém não modela o efeito "snap-back", pois não se implantou técnica específica. Para problemas desse tipo, que podem aparecer na Mecânica do Dano, usa-se a técnica do comprimento de arco (Crisfield, 1991) sendo que tal técnica foi aplicada à formulação implícita do MEC,(Botta, 2003), (Botta; Venturini ; Benallal, 2005b), (Botta; Venturini ; Benallal, 2005a), (Fernandes ; Venturini, 2002),(Fernandes, 1998), (Fudoli, 1999) e (Benallal; Botta ; Venturini, 2006).

\subsubsection{Mecânica da Fratura Elástica Linear utilizando o MEC}

No campo da Mecânica da Fratura, podem ser citados primeiramente os trabalhos de (Griffith, 1921) e (Irwin, 1957), onde se descrevem o equilíbrio energético de um sólido contendo descontinuidades, e a formulação dos fatores de tensão que permitem avaliar a tensão nas imediações do início da fratura.

Os primeiros resultados numéricos relativos a problemas de mecânica do fraturamento linear a partir do uso do MEC foram publicados há mais de 20 anos, por (Cruse, 1970). Um segundo trabalho de autoria do mesmo autor (Cruse ; Vanburen, 1971) é publicado um ano mais tarde, trazendo uma discussão mais completa do uso do MEC para o problema.

As primeiras formulações e resultados têm apenas o mérito de iniciar o uso do método em problemas de fratura mecânica. Tais formulações levavam facilmente a problemas numéricos de singularidades devido às representações integrais apresentarem muitas semelhanças quando escritas para pontos próximos.

Uma estratégia efetiva para a modelagem do fraturamento é proposta no trabalho de (Blandford; Ingraffea ; Ligget, 1981), onde é utilizada a clássica divisão do domínio em sub-regiões, fazendo com que a linha da fratura coincida com uma interface. Para esse tipo de formulação é necessário refazerem-se as malhas e consequentemente recalcularem-se as matrizes. São utilizadas apenas equações de deslocamento, portanto fortemente singulares, porém não há hipersingularidades. Nesse caso, as condições de separação são impostas aos nós da interface em função da solicitação calculada.

Mais recentemente, é interessante ressaltar o procedimento denominado "Dual Boundary Element Method"(DBEM), proposto por (Portela; Aliabadi ; Rooke, 
1992). Esse procedimento mostrou-se geral e computacionalmente eficiente para a modelagem de fraturas em problemas bi e tridimensionais. São agora empregadas equações integrais singulares, representações de deslocamento, e hipersingulares, representações de forças de superfície na linha de fratura.

Vários outros trabalhos, que utilizaram procedimentos similares para problemas bi e tridimensionais, poderiam ser citados (Watson, 1986), (Gray ; Giles, 1988), (Rudolphi; Krishnasamy; Scchmerr ; Rizzo, 1988) e (Gray; Martha ; Ingraffea, 1990). Nos últimos anos muitos modelos têm sido propostos para a modelagem de fratura mecânica associada ao MEC, considerando-se análises linear e não-linear. Um panorama mais completo do desenvolvimento de modelos de fratura via elementos de contorno pode ser visto nas seguintes referências (Cruse, 1988), (Aliabadi ; Rooke, 1991), (Aliabadi ; Brebbia, 1996) e (Cruse, 2004). Deve-se ainda destacar que os modelos que envolvem fratura mecânica são baseados tanto em formulações hipersingulares ou singulares, para os casos de problemas bidimensionais e tridimensionais. Ainda no campo da mecânica da fratura, mais recentemente destacam-se os modelos discretos da fratura (Maciel, 2003), onde devido à maior precisão das integrais analíticas, a fratura é discretizada como um domínio finito. Merecem serem citados na EESC os trabalhos de (Barbirato, 1999), (Pereira, 2004), (Lopes, 1996), (Lovón, 2006) e (Jiang ; Venturini, 1998).

\subsubsection{Mecânica da Fratura Não-Linear}

A formulação não linear na mecânica da fratura modela a propagação e o direcionamento da fratura utilizando-se de modelos de energia escritos para incrementos de tensões finitas. Com o incremento das solicitações, aparecem regiões com características mecânicas deterioradas na frente da ponta da fratura. Nos metais o processo não linear predominante é a plastificação enquanto que no concreto tem-se a predominância da formação de microfissuras.

Com o desenvolvimento de novos métodos numéricos, tais como o método dos elementos finitos generalizados, X-FEM e outros, tornou-se mais fácil e prático discretizar sólidos complexos e com regiões de descontinuidades, podendo-se citar vários trabalhos no campo de propagação de fraturas, tais como (Sukumar; Chopp ; Moran, 2003), onde é utilizado o X-FEM para análise de propagação de fraturas 
em sólidos tridimensionais, (Dolbow ; Nadeau, 2002) e (Dolbow; Moes ; Belytschko, 2001), onde é analisada pelo X-FEM a propagação de fraturas para materiais microestruturados como o concreto, e para análise de fraturas onde podem aparecer regiões com contato. No campo do desenvolvimento do método dos elementos finitos sem malhas e generalizados, pode-se citar na EESC os trabalhos de (Barros, 2002), (Torres, 2003) e (Pereira, 2004).

Outro tipo de análise de propagação de fraturas é a técnica de mudança de malha ou 'remeshing', onde a discretização do sólido é constantemente alterada com a progressão da fratura. Citam-se os trabalhos de (Komori, 2001) utilizando MEF e (Schollmann; Fulland ; Richard, 2003) via X-MEF. Tal técnica produz um aumento sensível no trabalho computacional pois a malha e as equações de equilíbrio são refeitas constantemente.

\subsubsection{Mecânica do Dano Contínuo}

Diversos trabalhos têm sido publicados sobre teoria do dano contínuo para simular o comportamento do concreto.

Deve-se a (Kachanov, 1958) o primeiro trabalho introduzindo o conceito de dano. Suas pesquisas abordaram problemas de fluência uniaxial para metais submetidos a altas temperaturas, e introduziu a variável de dano, para descrever a capacidade de uma seção transversal transmitir carga. A mecânica do dano contínuo foi formalizada com base na termodinâmica dos processos irreversíveis (Lemaitre ; Chaboche, 1985).

A utilização do Método dos Elementos de Contorno para análise não-linear de modelos de dano é muito recente, podendo serem citados os trabalhos de (Botta, 2003) e (Benallal ; Venturini, 2004). Nos modelos de dano há um complicador a mais para a análise numérica. É que neste caso as características mecânicas do material, como o módulo de elasticidade, se alteram, dando origem a curvas com comportamento de 'snap-back', necessitando de novas formas de previsão. A mais utilizada é o comprimento de arco, já comentada.

Dois modelos usuais de dano e convenientes para a elaboração de modelos mecânicos são os propostos por (Mazars, 1984), (Mazars, 1986), e (Borderie; PijaudierCabot ; Mazars, 1992). São critérios bastante simples, porém, já conseguem repre- 
sentar o comportamento do concreto razoavelmente.

Utilizando-se tais modelos, alguns trabalhos de mestrado e doutorado já foram desenvolvidos na EESC e na Escola Politécnica da USP: (Alvares, 1993), (Alvares, 1999), (Pituba, 2003), (Pituba, 1998), (Gonçalves, 2003), (Balbo, 1998), (Driemeier, 1995), (Driemeier, 1999), (Botta, 1998) e (Bussamra, 1993). Será utilizado, também neste trabalho, o modelo de dano local bidissipativo (Comi ; Perego, 2000), onde o sólido terá comportamento e patamares de danificação diferentes na tração e na compressão, como ocorre na realidade no concreto.

\subsubsection{Evolução da Fratura através de Processo de Danificação}

No trabalho proposto aqui, dedicado a materiais cimentícios, a fratura se forma com o surgimento de uma pequena e estreita zona anelástica, onde ocorrem os fenômenos de microfissuramentos, com a conseqüente dissipação de energia. Essa zona, chamada zona de processo (Cedolin; Poli ; Iori, 1987), antecipa a separação do sólido (abertura da fratura), fazendo com que o material dessa região perca rigidez. Esse modelo simples, denominado modelo de fratura coesiva, já é conhecido há muito tempo, como mostram os trabalhos pioneiros de (Baremblatt, 1962), e de seus seguidores como (Hillerborg; Modeer ; Peterson, 1976) e (Carpinteri, 1992). O modelo coesivo aparece em várias formulações do MEC (Liang ; Li, 2004) e (Cen ; Maier, 1992), onde a simulação da abertura da fratura é feita baseada na técnica das sub regiões. Também utilizando a formulação simétrica do MEC, o modelo coesivo já foi testado (Maier; Novati ; Cen, 1993).

Citam-se também neste tipo de análise os trabalhos de (Manzoli, 1998) e (Benallal ; Venturini, 2004), onde é feita, com a utilização do MEF, uma formulação onde se consideram equações que incorporam descontinuidades de deslocamentos nas funções aproximadoras, o que ocorre quando as deformações vão se localizando em uma faixa cada vez mais estreita, até se concretizar a descontinuidade nos deslocamentos. Na EESC pode ser citado o trabalho de (Gonçalves, 2003). 


\section{TEORIA DA ELASTICIDADE}

\subsection{Introdução}

Neste capítulo serão mostradas as formulações básicas da teoria da elasticidade. A formulação numérica está baseada na elasticidade plana, onde se estuda o comportamento mecânico dos sólidos em duas dimensões.

Primeiramente será discutida a definição de tensão, seu plano de atuação e a relação de tensão com forças de superfície. Posteriormente, na mesma linha, será estudada a formulação de deformações, seu plano de atuação e a relação entre deformação e deslocamento.

Numa segunda etapa, será mostrada a relação entre tensão e deformação em uma formulação puramente elástica, ou seja, a Lei de Hooke. O sólido será considerado como isotrópico, o que diminui o número das constantes elásticas do material e simplifica sua análise numérica. Será vista também a formulação da equação de Navier, onde o comportamento do sólido está resumido em apenas uma equação.

A formulação elástica do material será representada em duas dimensões, de onde pode-se chegar a um estado plano de tensão ou de deformação.

Por último, será mostrada a rotação de tensão e deformação para eixos principais e para eixos ortogonais quaisquer, que será utilizada no desenvolvimento deste trabalho.

\subsection{Leis constitutivas elásticas}

\subsubsection{Tensão}

Na natureza uma força dita pontual nunca ocorre. Forças são sempre distribuídas em superfícies finitas ou no volume do sólido. A figura (2.1) mostra um determinado corpo, onde foi feito um corte por um plano fictício $Q$, aparecendo uma 
das superfície $A$ no plano de corte $Q$, sobre a qual estão atuando forças de contato que a une com a outra superfície de corte.

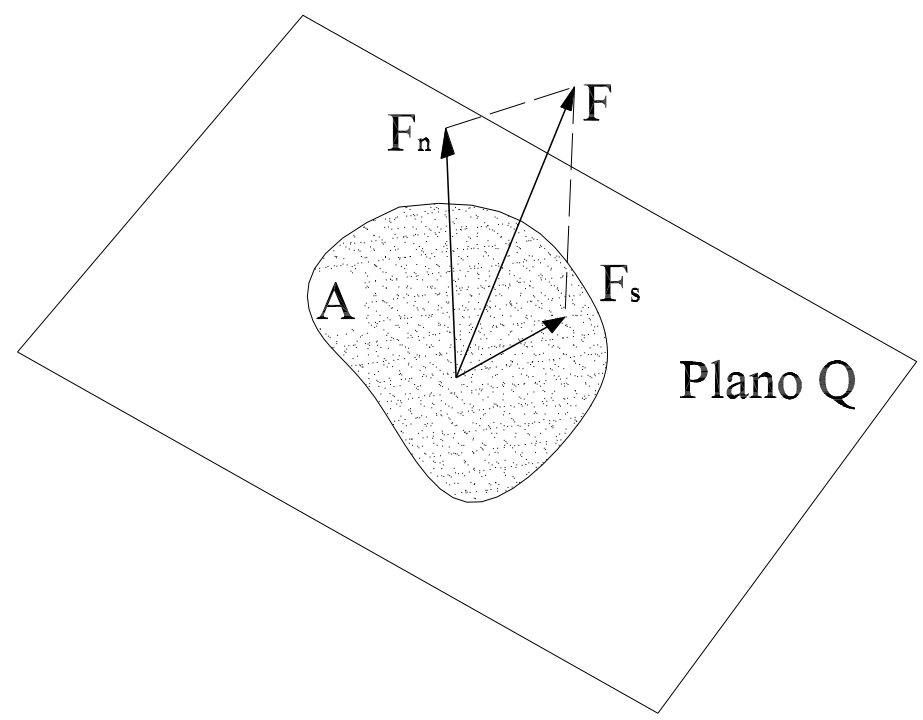

Fig. 2.1: Força de contato atuando em um determinado plano de corte

Nota-se que, uma das forças pode ser decomposta em uma força $F_{n}$, atuando na direção normal ao plano de atuação, e em uma força $F_{s}$, atuando tangencialmente. Naturalmente em um processo de deformação, a influência da força $F$ vai depender da variação da superfície $A$. A magnitude média das forças $F_{n}$ e $F_{s}$, por área, é dada por $F_{n} / A$ e $F_{s} / A$, sendo que, essas taxas são denominadas de tensão normal e tensão cisalhante, respectivamente. A dimensão física de tensão, para um ponto, é estabelecida levando-se a área infinitesimal a zero. Neste processo, as forças $F_{n}$ e $F_{s}$ também tendem a zero, levando as relações $F_{n} / A$ e $F_{s} / A$ a limites diferentes de zero. Os valores limites da taxa $F_{n} / A$ e $F_{s} / A$, são chamados de tensão normal e tensão tangencial de um ponto. Matematicamente pode-se escrever:

$$
\lim _{A \rightarrow 0} \frac{F_{n}}{A}=\sigma \quad \quad \lim _{A \rightarrow 0} \frac{F_{s}}{A}=\tau
$$

Como pode ser visto na figura (2.1), a tensão depende também do plano onde 
está área está contida. Em relação às coordenadas cartesianas, podem ser escritas as seguintes componentes de tensões:

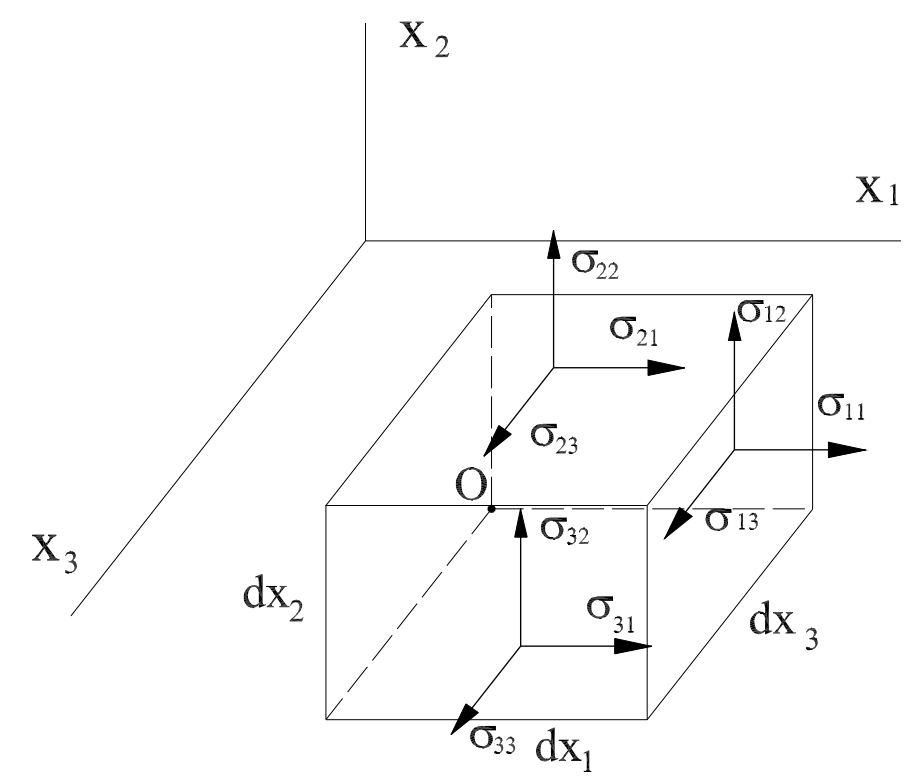

Fig. 2.2: Tensões nas faces normais aos eixos cartesianos

$$
\left[\begin{array}{lll}
\sigma_{11} & \sigma_{12} & \sigma_{13} \\
\sigma_{21} & \sigma_{22} & \sigma_{23} \\
\sigma_{31} & \sigma_{32} & \sigma_{33}
\end{array}\right]
$$

Na representação matricial do estado de tensões (2.2), o primeiro índice diz respeito ao plano onde está atuando a tensão, e o segundo índice se refere a direção da tensão. Aparentemente, nove componentes de tensões são necessárias para definir o estado de tensão de um ponto.

No elemento infinitesimal da figura (2.3), considerando-se a variação da tensão com apenas o primeiro termo da série de Taylor, e analisando apenas a variação das tensões na direção $x_{1}$, por exemplo, tem-se a seguinte equação de equilíbrio de forças nesta direção. 


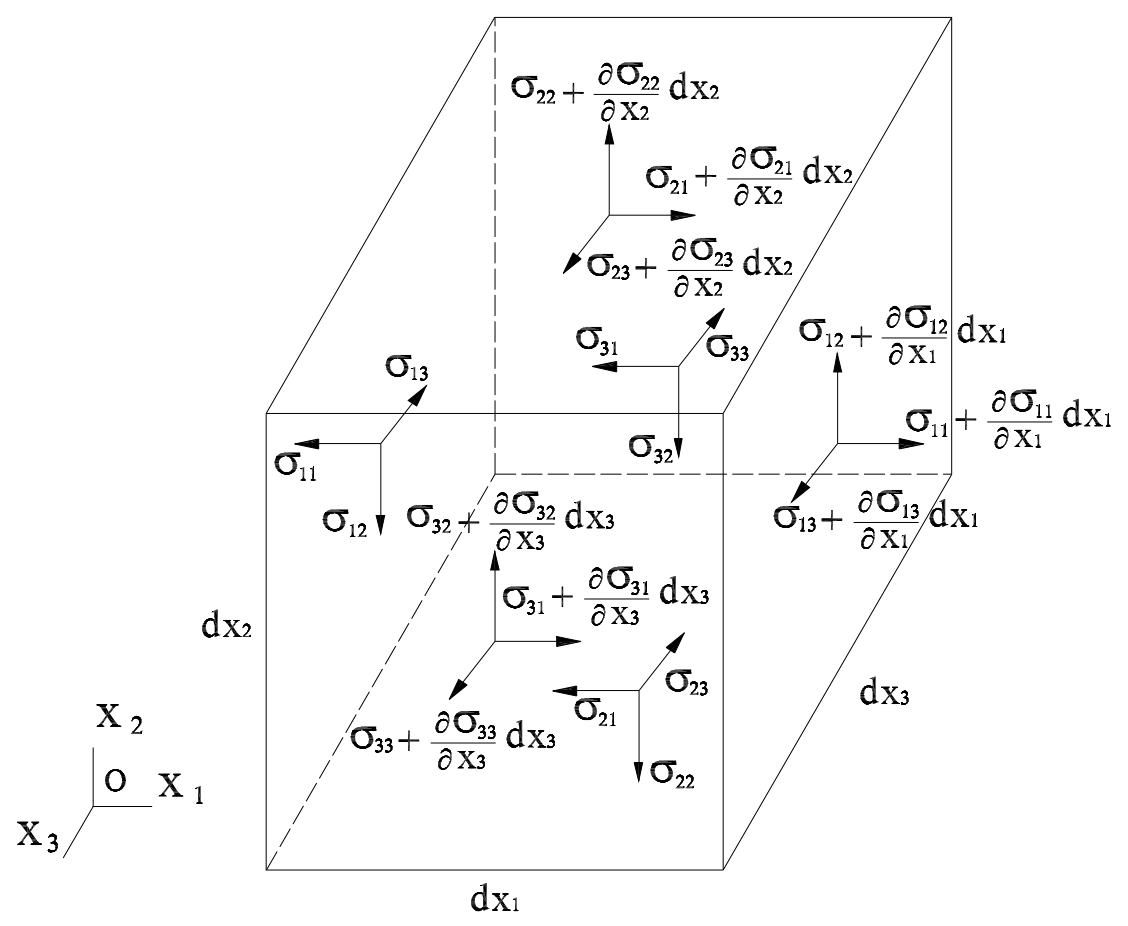

Fig. 2.3: Variação das tensões no elemento infinitesimal

$$
\begin{aligned}
& \Sigma F_{x_{1}}=-\sigma_{11} d x_{2} d x_{3}-\sigma_{21} d x_{1} d x_{2}-\sigma_{31} d x_{1} d x_{3}+\left(\sigma_{11}+\frac{\partial \sigma_{11}}{\partial x_{1}} d x_{1}\right) d x_{2} d x_{3}+ \\
& +\left(\sigma_{21}+\frac{\partial \sigma_{21}}{\partial x_{2}} d x_{2}\right) d x_{3} d x_{1}+\left(\sigma_{31}+\frac{\partial \sigma_{31}}{\partial x_{3}} d x_{3}\right) d x_{1} d x_{2}+f_{1} d x_{1} d x_{2} d x_{3}=0
\end{aligned}
$$

ou:

$$
\frac{\partial \sigma_{11}}{\partial x_{1}}+\frac{\partial \sigma_{21}}{\partial x_{2}}+\frac{\partial \sigma_{31}}{\partial x_{3}}+f_{1}=0
$$

sendo $f_{1}$, a força por unidade de volume atuando na direção $x_{1}$. Escrevendo (2.4) para todas as direções, em notação indicial.

$$
\sigma_{j i, j}+f_{i}=0
$$


onde os termos $f_{i}$ são as forças de volume nas direções dos eixos ortogonais cartesianos e $i, j=1,2,3$. Entretanto, considerando o elemento infinitesimal da figura (2.2), com as componentes de tensões representadas em todas as faces do elemento, e a ausência de momentos distribuídos, o somatório de momentos em torno do ponto $O$ deve ser nulo, para que o elemento esteja em equilíbrio estático.

$$
\begin{aligned}
& M_{x 1}=\left(\sigma_{23} d x_{1} d x_{3}\right) d x_{2}-\left(\sigma_{32} d x_{1} d x_{2}\right) d x_{3}=0 \\
& M_{x 2}=\left(\sigma_{31} d x_{1} d x_{2}\right) d x_{3}-\left(\sigma_{13} d x_{2} d x_{3}\right) d x_{1}=0 \\
& M_{x 3}=\left(\sigma_{12} d x_{2} d x_{3}\right) d x_{1}-\left(\sigma_{21} d x_{1} d x_{3}\right) d x_{2}=0
\end{aligned}
$$

Deduz-se da equação de equilíbrio (2.6), a seguinte relação de identidade de tensões:

$$
\sigma_{23}=\sigma_{32} \quad \sigma_{31}=\sigma_{13} \quad \sigma_{12}=\sigma_{21}
$$

portanto, são agora necessárias apenas 6 componentes de tensão, para definir o estado de tensão em um ponto.

Para um ponto situado na superfície de um corpo, como mostra a figura (2.4), pode-se escrever a equação de equilíbrio utilizando-se da fórmula de Cauchy:

$$
\begin{aligned}
& P_{x_{1}}=\eta_{1} \sigma_{11}+\eta_{2} \sigma_{12}+\eta_{3} \sigma_{13} \\
& P_{x_{2}}=\eta_{1} \sigma_{12}+\eta_{2} \sigma_{22}+\eta_{3} \sigma_{23} \\
& P_{x_{3}}=\eta_{1} \sigma_{13}+\eta_{2} \sigma_{23}+\eta_{3} \sigma_{33}
\end{aligned}
$$




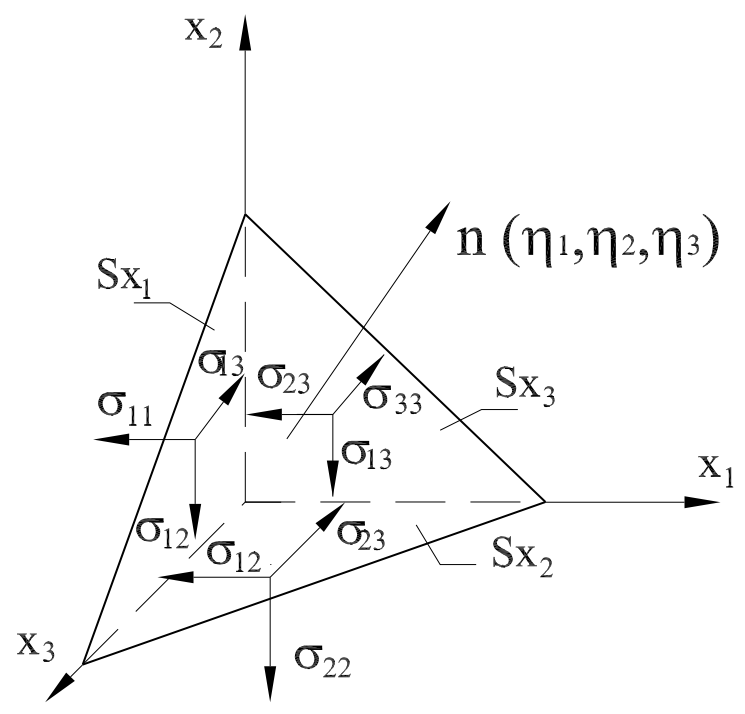

Fig. 2.4: Tensões atuantes nas faces do elemento de superfície definida pela normal $\mathbf{n}$

Onde $P_{x_{1}}, P_{x_{2}}$ e $P_{x_{3}}$ são as forças de superfície atuantes nas direções $x_{1}, x_{2}$, e $x_{3}$ e $\eta_{i}, i=1,2,3$, são os cossenos diretores da normal $n$ em relação aos eixos $x_{1}, x_{2}$ e $x_{3}$, respectivamente. Pode-se então escrever a equação (2.8) matricialmente:

$$
\left[\begin{array}{c}
P_{x_{1}} \\
P_{x_{2}} \\
P_{x_{3}}
\end{array}\right]=\left[\begin{array}{ccc}
\sigma_{11} & \sigma_{12} & \sigma_{13} \\
\sigma_{12} & \sigma_{22} & \sigma_{23} \\
\sigma_{13} & \sigma_{23} & \sigma_{33}
\end{array}\right] \cdot\left[\begin{array}{l}
\eta_{1} \\
\eta_{2} \\
\eta_{3}
\end{array}\right]
$$

A figura (2.5) mostra a relação dos eixos $x_{1}, x_{2}, x_{3}$ em relação ao eixo $\bar{x}_{1}$, dada pelos ângulos $\alpha_{11}, \alpha_{21}$ e $\alpha_{31}$. O primeiro índice do ângulo $\alpha$ diz respeito aos eixos cartesianos, e o segundo índice, ao sistema de eixos $\bar{x}_{1}, \bar{x}_{2}, \bar{x}_{3}$.

Analogamente, pode-se escrever a relação entre os eixos cartesianos e os eixos $\bar{x}_{2}$ e $\bar{x}_{3}$, através dos ângulos $\alpha_{12}, \alpha_{22}, \alpha_{32}$ e $\alpha_{13}, \alpha_{23}, \alpha_{33}$ respectivamente, podendo-se escrever matricialmente:

$$
\left[\begin{array}{l}
x_{1} \\
x_{2} \\
x_{3}
\end{array}\right]=\left[\begin{array}{lll}
\alpha_{11} & \alpha_{21} & \alpha_{31} \\
\alpha_{12} & \alpha_{22} & \alpha_{32} \\
\alpha_{13} & \alpha_{23} & \alpha_{33}
\end{array}\right]\left[\begin{array}{l}
\bar{x}_{1} \\
\bar{x}_{2} \\
\bar{x}_{3}
\end{array}\right] \quad\left[\begin{array}{l}
x_{1} \\
x_{2} \\
x_{3}
\end{array}\right]=[R]\left[\begin{array}{l}
\bar{x}_{1} \\
\bar{x}_{2} \\
\bar{x}_{3}
\end{array}\right]
$$




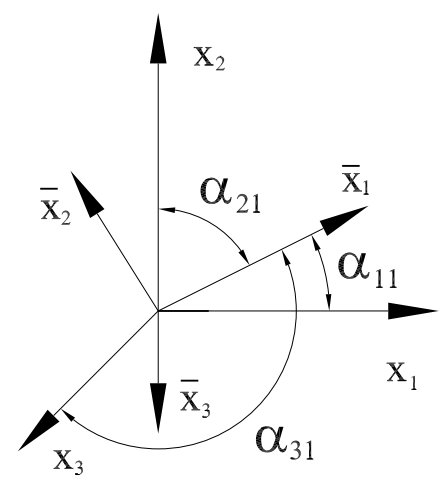

Fig. 2.5: Relação entre o sistema ortogonal $x_{1}, x_{2}, x_{3}$ e o eixo $\bar{x}_{1}$.

Utilizando-se de (2.10), podem-se encontrar as forças de superfície atuantes segundo sistema de eixos $\bar{x}_{1}, \bar{x}_{2}, \bar{x}_{3}$.

$$
\left[\begin{array}{l}
P_{x_{1}} \\
P_{x_{2}} \\
P_{x_{3}}
\end{array}\right]=\left[\begin{array}{lll}
\alpha_{11} & \alpha_{21} & \alpha_{31} \\
\alpha_{12} & \alpha_{22} & \alpha_{32} \\
\alpha_{13} & \alpha_{23} & \alpha_{33}
\end{array}\right]\left[\begin{array}{c}
P_{\bar{x}_{1}} \\
P_{\bar{x}_{2}} \\
P_{\bar{x}_{3}}
\end{array}\right]
$$

Pode-se também rotacionar as forças atuantes no sistema cartesiano para o sistema $\bar{x}_{1}, \bar{x}_{2}, \bar{x}_{3}$, pela relação:

$$
\left[\begin{array}{c}
P_{\bar{x}_{1}} \\
P_{\bar{x}_{2}} \\
P_{\bar{x}_{3}}
\end{array}\right]=\left[\begin{array}{lll}
\alpha_{11} & \alpha_{12} & \alpha_{13} \\
\alpha_{21} & \alpha_{22} & \alpha_{23} \\
\alpha_{31} & \alpha_{32} & \alpha_{33}
\end{array}\right]\left[\begin{array}{c}
P_{x_{1}} \\
P_{x_{2}} \\
P_{x_{3}}
\end{array}\right] \quad \text { ou } \quad\left[\begin{array}{c}
P_{\bar{x}_{1}} \\
P_{\bar{x}_{2}} \\
P_{\bar{x}_{3}}
\end{array}\right]=[R]^{T}\left[\begin{array}{l}
P_{x_{1}} \\
P_{x_{2}} \\
P_{x_{3}}
\end{array}\right]
$$

Considerando um plano perpendicular ao eixo $\bar{x}$, pode-se escrever para um ponto pertencente a esse plano, a seguinte equação de equilíbrio:

$$
\left[\begin{array}{l}
P_{\bar{x}_{1}} \\
P_{\bar{x}_{2}} \\
P_{\bar{x}_{3}}
\end{array}\right]=\left[\begin{array}{l}
\bar{\sigma}_{11} \\
\bar{\sigma}_{12} \\
\bar{\sigma}_{13}
\end{array}\right]
$$


Com isto, utilizando de (2.13) em (2.12), e escrevendo as forças de superfície da equação (2.12) segundo o teorema de Cauchy, obtém-se:

$$
\left[\begin{array}{l}
\bar{\sigma}_{11} \\
\bar{\sigma}_{12} \\
\bar{\sigma}_{13}
\end{array}\right]=\left[\begin{array}{lll}
\alpha_{11} & \alpha_{12} & \alpha_{13} \\
\alpha_{21} & \alpha_{22} & \alpha_{23} \\
\alpha_{31} & \alpha_{32} & \alpha_{33}
\end{array}\right]\left[\begin{array}{lll}
\sigma_{11} & \sigma_{12} & \sigma_{13} \\
\sigma_{12} & \sigma_{22} & \sigma_{23} \\
\sigma_{13} & \sigma_{23} & \sigma_{33}
\end{array}\right] \cdot\left[\begin{array}{l}
\alpha_{11} \\
\alpha_{12} \\
\alpha_{13}
\end{array}\right]
$$

Usando o mesmo procedimento para os eixos $\bar{x}_{2}$ e $\bar{x}_{3}$, tem-se de forma geral:

$$
\left[\begin{array}{ccc}
\bar{\sigma}_{11} & \bar{\sigma}_{12} & \bar{\sigma}_{13} \\
\bar{\sigma}_{12} & \bar{\sigma}_{22} & \bar{\sigma}_{23} \\
\bar{\sigma}_{13} & \bar{\sigma}_{12} & \bar{\sigma}_{33}
\end{array}\right]=\left[\begin{array}{lll}
\alpha_{11} & \alpha_{12} & \alpha_{13} \\
\alpha_{21} & \alpha_{22} & \alpha_{23} \\
\alpha_{31} & \alpha_{32} & \alpha_{33}
\end{array}\right]\left[\begin{array}{lll}
\sigma_{11} & \sigma_{12} & \sigma_{13} \\
\sigma_{12} & \sigma_{22} & \sigma_{23} \\
\sigma_{13} & \sigma_{23} & \sigma_{33}
\end{array}\right]\left[\begin{array}{lll}
\alpha_{11} & \alpha_{21} & \alpha_{31} \\
\alpha_{12} & \alpha_{22} & \alpha_{32} \\
\alpha_{13} & \alpha_{23} & \alpha_{33}
\end{array}\right]
$$

Escrevendo a equação (2.15) na forma indicial:

$$
\bar{\sigma}_{k m}=\alpha_{k j} \sigma_{j i} \alpha_{i m} \quad k, m, j, i=1,2,3
$$

Para um ponto $P$ em um meio contínuo, existem três planos perpendiculares entre si, nos quais as tensões cisalhantes $\sigma_{i j}(i \neq j)$ são nulas, onde só atuam tensões normais. Essas tensões são chamadas de tensões principais e, os eixos que as contém, de eixos principais de tensões.

Seja $\hat{\mathbf{n}}$ o vetor da direção dos eixos principais e $\lambda$ as tensões principais orientadas segundo a direção dos eixos principais. Considerando a nulidade das tensões tangenciais nesta direção, tem-se:

$$
\sigma_{i i}=\lambda \hat{\mathbf{n}}
$$


ou segundo a equação (2.13):

$$
n_{r} \sigma_{r s}=\lambda n_{s} \quad \text { onde: } r, s=1,2,3
$$

ou:

$$
\left(\sigma_{r s}-\lambda \delta_{r s}\right) n_{r}=0
$$

e, em notação matricial:

$$
n\left(\sigma_{r s}-\lambda I\right)=0
$$

onde I é a matriz identidade; a equação (2.19) é um conjunto de três equações algébricas homogêneas lineares, para as direções dos cossenos diretores $n_{1}, n_{2}$ e $n_{3}$, as quais ainda devem satisfazer a relação:

$$
n_{1}^{2}+n_{2}^{2}+n_{3}^{2}=1
$$

sendo as tensões principais multiplicadas pela matriz identidade $\boldsymbol{I}$, como se apresenta na equação (2.20), dadas por:

$$
\lambda \mathbf{I}=\left[\begin{array}{lll}
\lambda & 0 & 0 \\
0 & \lambda & 0 \\
0 & 0 & \lambda
\end{array}\right]
$$


Para satisfazer a equação (2.22), os três cossenos diretores não podem ser todos nulos. O sistema de equações lineares da equação (2.20), tem solução se o determinante dos coeficientes $\left(\sigma_{r s}-\lambda I\right)$ for igual a zero:

$$
\left|\sigma_{r s}-\lambda \mathbf{I}\right|=\left|\begin{array}{ccc}
\sigma_{11}-\lambda & \sigma_{12} & \sigma_{13} \\
\sigma_{12} & \sigma_{22}-\lambda & \sigma_{23} \\
\sigma_{13} & \sigma_{23} & \sigma_{33}-\lambda
\end{array}\right|=0
$$

Resulta uma equação cúbica contendos os termos invariantes, os quais tem este nome, pois possuem os mesmos valores, independentemente da orientação do sistema de eixos coordenados adotado:

$$
\lambda^{3}-I_{1} \lambda^{2}+I_{2} \lambda-I_{3}=0
$$

onde:

$$
\begin{array}{r}
I_{1}=\sigma_{11}+\sigma_{22}+\sigma_{33} \\
I_{2}=\left|\begin{array}{ll}
\sigma_{11} & \sigma_{12} \\
\sigma_{12} & \sigma_{22}
\end{array}\right|+\left|\begin{array}{ll}
\sigma_{11} & \sigma_{13} \\
\sigma_{13} & \sigma_{33}
\end{array}\right|+\left|\begin{array}{cc}
\sigma_{22} & \sigma_{23} \\
\sigma_{23} & \sigma_{33}
\end{array}\right| \\
I_{3}=\left|\begin{array}{ccc}
\sigma_{11} & \sigma_{12} & \sigma_{13} \\
\sigma_{12} & \sigma_{22} & \sigma_{23} \\
\sigma_{13} & \sigma_{23} & \sigma_{33}
\end{array}\right|
\end{array}
$$

As três raízes da equação (2.24) são as tensões principais normais, contidas nos eixos de mesma nomenclatura. Como as tensões principais são encontradas em função dos termos invariantes da equação (2.25), são também invariantes e independem da escolha do sistema de coordenadas. 


\subsubsection{Deformação}

Para alguns propósitos da mecânica clássica, um corpo pode ser admitido como rígido, isto é, não sofre modificações em sua geometria após ser solicitado por algum tipo de força. Fisicamente isto é impossível, pois todo sólido se deforma em maior ou menor grau.

Tal fenômeno pode ser tratado como pequenas deformações, onde as deformações são de tal ordem que o sólido pode ser analisado mecanicamente em sua posição inicial. Esta hipótese é considerada na maioria dos materiais empregados na Engenharia Civil, pois pela magnitude dos esforços e da resistência mecânica dos materiais, as deformações são muito pequenas.

Outro tipo de análise a ser feita é o caso das grandes deformações, onde a posição de equilíbrio do corpo deve ser considerada na posição atual do sólido, isto é, depois de sofrer as modificações impostas pelas forças aplicadas. Este é o caso da conformação mecânica dos metais e de materiais muito deformáveis, utilizados principalmente na indústria aeronáutica. Neste trabalho será tratada apenas a teoria das pequenas deformações.

\subsubsection{Pequenas deformações e rotações em duas dimensões}

A figura (2.6) mostra um elemento infinitesimal sujeito a deformações.
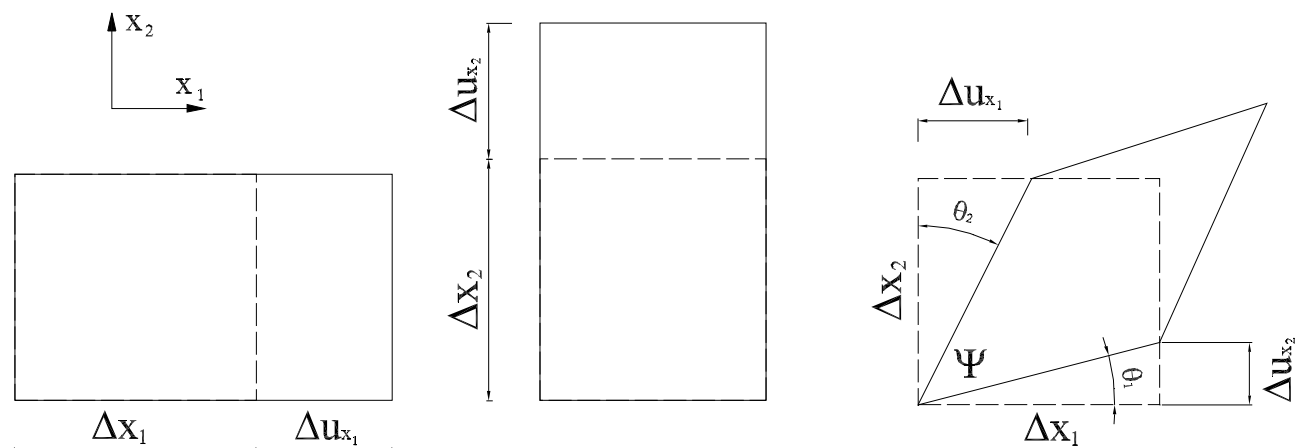

Fig. 2.6: Deformações axiais e angulares 


$$
\begin{array}{r}
\epsilon_{11} \approx \frac{\Delta u_{x_{1}}}{\Delta x_{1}} \\
\epsilon_{12}=\frac{\Delta u_{x_{2}}}{\Delta x_{2}}-\Psi=\theta_{2}+\theta_{1} \\
\epsilon_{12} \approx \frac{1}{2}\left(\frac{\Delta u_{x_{1}}}{\Delta x_{2}}+\frac{\Delta u_{x_{2}}}{\Delta x_{1}}\right)
\end{array}
$$

onde $\epsilon_{11}$ e $\epsilon_{22}$ são definidos como a taxa de variação do comprimento pelo comprimento original nas direções $x_{1}$ e $x_{2}$, respectivamente, e $\epsilon_{12}$ é definido como a metade do decréscimo do ângulo inicial, $\gamma_{12}$, formado pelos lados paralelos aos eixos $x_{1}$ e $x_{2}$ da figura (2.6).

Então, por definição, entende-se por deformação como o processo físico, onde há mudanças relativas de posição entre partículas dentro de um sólido, seja em uma direção linear ou angular. E com esta alteração, o sólido apresenta-se com uma forma geométrica diferente da que apresentava anteriormente ao processo de deformação.

Os sinais de aproximado se devem ao fato de se tratarem de pequenas deformações, e esses resultados se aproximam satisfatoriamente da realidade. Tomando o limite de $\Delta x_{1}$ e $\Delta x_{2}$ da equação (2.26) tendendo a zero, tem-se:

$$
\epsilon_{11}=\frac{\partial u_{x_{1}}}{\partial x_{1}}, \quad \epsilon_{22}=\frac{\partial u_{x_{2}}}{\partial x_{2}}, \quad \epsilon_{12}=\frac{1}{2} \gamma_{12}=\frac{1}{2}\left(\frac{\partial u_{x_{1}}}{\partial x_{2}}+\frac{\partial u_{x_{2}}}{\partial x_{1}}\right)
$$

Ou seja, por definição os termos $\epsilon_{11}, \epsilon_{22}$ e $\epsilon_{12}$ são as taxas de deslocamentos relativos longitudinais nas direções $x_{1}, x_{2}$ e deslocamentos angulares entre os pontos de um sólido, respectivamente.

Deve-se salientar que, nos deslocamentos de corpo rígido que os corpos possam apresentar, não há o processo de deformação, pois não há deslocamentos relativos entre as partículas. Este é o caso da rotação de corpo rígido, figura (2.7), onde tem-se as equações de rotações: 

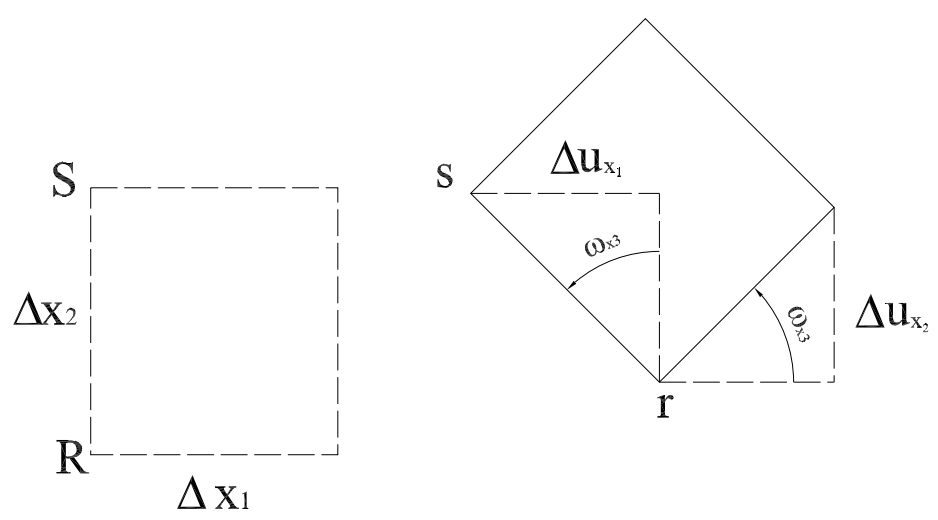

Fig. 2.7: Deslocamento e rotação rígida sobre o plano $x_{1}-x_{2}$

$$
\omega_{3}=\Omega_{12}=-\Omega_{21}=\frac{1}{2}\left(\frac{\partial u_{x_{2}}}{\partial x_{1}}-\frac{\partial u_{x_{1}}}{\partial x_{2}}\right)
$$

\subsubsection{Deslocamentos relativos em $2 D$}

Considerando-se dois pontos vizinhos $P$ e $Q$ em um plano de meio contínuo e, supondo que esses pontos sofram mudanças de posição para os pontos $p$ e $q$, respectivamente, como mostra a figura (2.8).

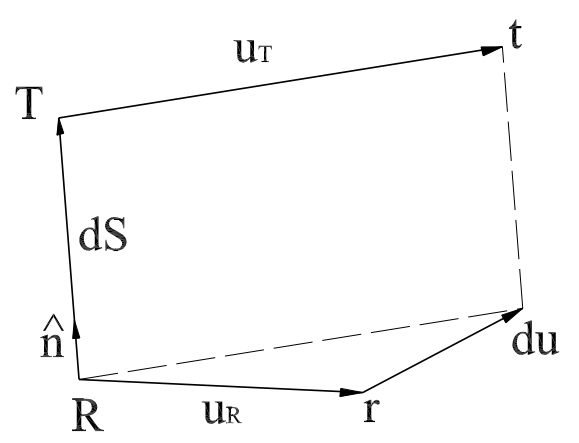

Fig. 2.8: Deslocamentos relativos entre os pontos $\mathrm{R}$ e $\mathrm{T}$

O termo $\boldsymbol{d} \boldsymbol{u}$ é a diferença entre os deslocamentos $\boldsymbol{u}_{\boldsymbol{T}}$ e $\boldsymbol{u}_{\boldsymbol{R}}$, enquanto que pode-se escrever a taxa da variação do deslocamento em relação ao comprimento 
inicial $d S$, como $\boldsymbol{d u} / \boldsymbol{d} \boldsymbol{S}$. As componentes retangulares das taxas de deslocamentos podem ser escritas por:

$$
\frac{d u_{x_{1}}}{d S}=\frac{\partial u_{x_{1}}}{\partial x_{1}} \frac{d x_{1}}{d S}+\frac{\partial u_{x_{1}}}{d x_{2}} \frac{d x_{2}}{d S}, \quad \frac{d u_{x_{2}}}{d S}=\frac{\partial u_{x_{2}}}{\partial x_{1}} \frac{d x_{1}}{d S}+\frac{\partial u_{x_{2}}}{d x_{2}} \frac{d x_{2}}{d S}
$$

ou matricialmente:

$$
\left[\begin{array}{l}
\frac{d u_{x_{1}}}{d S} \\
\frac{d u_{x_{2}}}{d S}
\end{array}\right]=\left[\begin{array}{ll}
\frac{\partial u_{x_{1}}}{\partial x_{1}} & \frac{\partial u_{x_{1}}}{\partial x_{2}} \\
\frac{\partial u_{x_{2}}}{\partial x_{1}} & \frac{\partial u_{x_{2}}}{\partial x_{2}}
\end{array}\right]\left[\begin{array}{l}
\frac{d x_{1}}{d S} \\
\frac{d x_{2}}{d S}
\end{array}\right]
$$

Os termos da matriz de deslocamentos relativos podem ser separados em duas parcelas, sendo a primeira relativa a deformações propriamente ditas e a segunda, referente a deslocamento de corpo rígido devido à rotação:

$$
\begin{aligned}
{\left[\begin{array}{ll}
\frac{\partial u_{x_{1}}}{\partial x_{1}} & \frac{\partial u_{x_{1}}}{\partial x_{2}} \\
\frac{\partial u_{x_{2}}}{\partial x_{1}} & \frac{\partial u_{x_{2}}}{\partial x_{2}}
\end{array}\right]=} & {\left[\begin{array}{cc}
\frac{\partial u_{x_{1}}}{\partial x_{1}} & \frac{1}{2}\left(\frac{\partial u_{x_{1}}}{\partial x_{2}}+\frac{\partial u_{x_{2}}}{\partial x_{1}}\right) \\
\frac{1}{2}\left(\frac{\partial u_{x_{2}}}{\partial x_{1}}+\frac{\partial u_{x_{1}}}{\partial x_{2}}\right) & \frac{\partial u_{x_{2}}}{\partial x_{2}}
\end{array}\right]+} \\
& +\left[\begin{array}{cc}
0 & \frac{1}{2}\left(\frac{\partial u_{x_{1}}}{\partial x_{2}}-\frac{\partial u_{x_{2}}}{\partial x_{1}}\right) \\
\frac{1}{2}\left(\frac{\partial u_{x_{2}}}{\partial x_{1}}-\frac{\partial u_{x_{1}}}{\partial x_{2}}\right) & 0
\end{array}\right]
\end{aligned}
$$

onde:

$$
\Omega_{12}=\frac{1}{2}\left(\frac{\partial u_{x_{1}}}{\partial x_{2}}-\frac{\partial u_{x_{2}}}{\partial x_{1}}\right), \quad \Omega_{21}=-\Omega_{12}=\frac{1}{2}\left(\frac{\partial u_{x_{2}}}{\partial x_{1}}-\frac{\partial u_{x_{1}}}{\partial x_{2}}\right)
$$




$$
\epsilon_{12}=\frac{1}{2}\left(\frac{\partial u_{x_{1}}}{\partial x_{2}}+\frac{\partial u_{x_{2}}}{\partial x_{1}}\right), \quad \epsilon_{21}=\epsilon_{12}=\frac{1}{2}\left(\frac{\partial u_{x_{2}}}{\partial x_{1}}+\frac{\partial u_{x_{1}}}{\partial x_{2}}\right)
$$

A equação matricial de deformação de um sólido, $\boldsymbol{J}_{\boldsymbol{u}}$, pode ser escrita como:

$$
J_{u}=E+\Omega
$$

sendo $\boldsymbol{E}$ a matriz de deformação e $\boldsymbol{\Omega}$ a matriz de rotação:

$$
\mathbf{E}=\left[\begin{array}{cc}
\epsilon_{11} & \epsilon_{12} \\
\epsilon_{21} & \epsilon_{22}
\end{array}\right], \quad \quad \boldsymbol{\Omega}=\left[\begin{array}{cc}
0 & \Omega_{12} \\
\Omega_{21} & 0
\end{array}\right]
$$

Para o caso de análise em três dimensões tem-se:

$$
\boldsymbol{E}=\left[\begin{array}{ccc}
\frac{\partial u_{x_{1}}}{\partial x_{1}} & \frac{1}{2}\left(\frac{\partial u_{x_{1}}}{\partial x_{2}}+\frac{\partial u_{x_{2}}}{\partial x_{1}}\right) & \frac{1}{2}\left(\frac{\partial u_{x_{1}}}{\partial x_{3}}+\frac{\partial u_{x_{3}}}{\partial x_{3}}\right) \\
\frac{1}{2}\left(\frac{\partial u_{x_{2}}}{\partial x_{1}}+\frac{\partial u_{x_{1}}}{\partial x_{2}}\right) & \frac{\partial u_{x_{2}}}{\partial x_{2}} & \frac{1}{2}\left(\frac{\partial u_{x_{2}}}{\partial x_{3}}+\frac{\partial u_{x_{3}}}{\partial x_{2}}\right) \\
\frac{1}{2}\left(\frac{\partial u_{x_{3}}}{\partial x_{1}}+\frac{\partial u_{x_{1}}}{\partial x_{3}}\right) & \frac{1}{2}\left(\frac{\partial u_{x_{3}}}{\partial x_{2}}+\frac{\partial u_{x_{2}}}{\partial x_{3}}\right) & \frac{\partial u_{x_{3}}}{\partial x_{3}}
\end{array}\right]
$$

e a matriz de rotação de corpo rígido:

$$
\boldsymbol{\Omega}=\left[\begin{array}{ccc}
0 & \frac{1}{2}\left(\frac{\partial u_{x_{1}}}{\partial x_{2}}-\frac{\partial u_{x_{2}}}{\partial x_{1}}\right) & \frac{1}{2}\left(\frac{\partial u_{x_{1}}}{\partial x_{3}}-\frac{\partial u_{x_{3}}}{\partial x_{1}}\right) \\
\frac{1}{2}\left(\frac{\partial u_{x_{2}}}{\partial x_{1}}-\frac{\partial u_{x_{1}}}{\partial x_{2}}\right) & 0 & \frac{1}{2}\left(\frac{\partial u_{x_{2}}}{\partial x_{3}}-\frac{\partial u_{x_{3}}}{\partial x_{2}}\right) \\
\frac{1}{2}\left(\frac{\partial u_{x_{3}}}{\partial x_{1}}-\frac{\partial u_{x_{1}}}{\partial x_{3}}\right) & \frac{1}{2}\left(\frac{\partial u_{x_{3}}}{\partial x_{2}}-\frac{\partial u_{x_{2}}}{\partial x_{3}}\right) & 0
\end{array}\right]
$$


Com os tensores escritos na forma indicial:

$$
\epsilon_{i j}=\frac{1}{2}\left(\frac{\partial u_{i}}{\partial x_{j}}+\frac{\partial u_{j}}{\partial x_{i}}\right) \quad \Omega_{i j}=\frac{1}{2}\left(\frac{\partial u_{i}}{\partial x_{j}}-\frac{\partial u_{j}}{\partial x_{i}}\right)
$$

sendo $i, j=1,2$ para análise bidimensional e $i, j=1,2,3$ para tridimensional.

\subsubsection{Deformações principais e mudança de coordenadas}

Comportamento análogo ao caso das tensões se apresenta no caso das deformações principais, isto é, apenas deformações axiais ocorrem nas direções principais, e que por isso são chamadas de deformações principais. Com isso para se encontrar as deformações principais deve-se, como na equação (2.23):

$$
\lambda \mathbf{I}=\left|\begin{array}{ccc}
\epsilon_{11}-\lambda & \epsilon_{12} & \epsilon_{13} \\
\epsilon_{12} & \epsilon_{22}-\lambda & \epsilon_{23} \\
\epsilon_{13} & \epsilon_{23} & \epsilon_{33}-\lambda
\end{array}\right|=0
$$

que é uma equação cúbica:

$$
\lambda^{3}-I_{1} \lambda^{2}+I_{2} \lambda-I_{3}=0
$$

contendo os invariantes:

$$
\begin{gathered}
I_{1}=\epsilon_{11}+\epsilon_{22}+\epsilon_{33} \\
I_{2}=\left|\begin{array}{ll}
\epsilon_{11} & \epsilon_{12} \\
\epsilon_{12} & \epsilon_{22}
\end{array}\right|+\left|\begin{array}{ll}
\epsilon_{11} & \epsilon_{13} \\
\epsilon_{13} & \epsilon_{33}
\end{array}\right|+\left|\begin{array}{cc}
\epsilon_{22} & \epsilon_{23} \\
\epsilon_{23} & \epsilon_{33}
\end{array}\right|
\end{gathered}
$$




$$
I_{3}=\left|\begin{array}{ccc}
\epsilon_{11} & \epsilon_{12} & \epsilon_{13} \\
\epsilon_{12} & \epsilon_{22} & \epsilon_{23} \\
\epsilon_{13} & \epsilon_{23} & \epsilon_{33}
\end{array}\right|
$$

sendo as raízes da equação (2.39) as deformações principais.

No caso de mudança de coordenadas, as deformações variam segundo as relações entre os ângulos da direção original com a nova direção, como no caso das tensões. Pode-se então escrever a equação de transformação de deformações entre dois sistemas de eixos ortogonais, como na equação (2.16):

$$
\bar{\epsilon}_{k m}=\alpha_{k j} \epsilon_{j i} \alpha_{i m} \quad k, m, j, i=1,2,3
$$

onde $\bar{\epsilon}_{k m}$, são as deformações no sistema rotacionado, o qual se relaciona com o sistema inicial pelos ângulos $\alpha_{k j}$.

\subsubsection{Equações Constitutivas}

Considerando-se o caso de pequenas deformações e também um comportamento puramente elástico do sólido, onde não haja dissipação de energia num processo de deformação, tem-se que a energia das forças externas é absorvida integralmente no sólido, pelo processo de deformação. Com isto, tem-se a relação entre tensão e deformação e a energia acumulada no corpo em um processo de deformação:

$$
\begin{array}{cc}
\sigma_{i j}=\frac{\partial U}{\partial \epsilon_{i j}} & \text { para } i=j \\
\sigma_{i j}=\frac{\partial U}{\partial 2 \epsilon_{i j}} & \text { para } i \neq j
\end{array}
$$

com $i, j=1,2,3$. A função $U$ representa a energia potencial por unidade de volume acumulada no corpo pela deformação, que é também chamada de função de densi- 
dade de energia de deformação. Adotando-se uma relação linear entre deformação e tensão, pode-se escrever:

$$
\boldsymbol{\sigma}_{\boldsymbol{m}}=C_{m n} \boldsymbol{\epsilon}_{\boldsymbol{n}} \quad m, n=1,2, \cdots 6
$$

onde:

$$
\begin{aligned}
& \sigma_{1}=\sigma_{11} \quad \sigma_{2}=\sigma_{22} \quad \sigma_{3}=\sigma_{33} \quad \sigma_{4}=\sigma_{23} \quad \sigma_{5}=\sigma_{13} \quad \sigma_{6}=\sigma_{12} \\
& \epsilon_{1}=\epsilon_{11} \quad \epsilon_{2}=\epsilon_{22} \quad \epsilon_{3}=\epsilon_{33} \quad \epsilon_{4}=2 \epsilon_{23} \quad \epsilon_{5}=2 \epsilon_{13} \quad \epsilon_{6}=2 \epsilon_{12}
\end{aligned}
$$

Os termos $C_{m n}$ são chamados de módulos elásticos e são independentes. Para o caso anisotrópico, o número de constantes $C_{m n}$ é de 36 , podendo cair para 21 considerando-se a equação (2.42). As constantes vão caindo se for considerado ainda o corpo como ortotrópico, e cai para 2 constantes se o sólido for considerado como isotrópico, isto é, possuir o mesmo comportamento em todas as direções.

\subsubsection{Lei de Hooke generalizada - Análise Isotrópica}

No caso isotrópico, pode-se considerar a relação tensão-deformação com apenas dois módulos elásticos, sendo um relacionado à direção normal à superfície em análise e outro referente à direção tangencial. Na direção normal, tem-se o módulo de elasticidade longitudinal $E$, que relaciona a tensão numa direção normal à superfície com a deformação sofrida nesta direção, e o módulo de elasticidade transversal $G$, que relaciona a tensão cisalhante na direção tangencial à superfície com a deformação angular sofrida nesta superfície. Nota-se também que no caso das tensões normais, ao mesmo tempo em que o sólido sofre deformação linear na direção da tensão, sofre também deformações lineares em outras direções, e considerando-se estas deformações, adotou-se o coeficiente de Poisson $\nu$. Deve-se salientar que estas propriedades são intrínsecas a cada tipo de material, e sua determinação é feita por processos experimentais. 
Com estas hipóteses, pode-se escrever:

$$
\begin{array}{r}
\epsilon_{11}=\frac{1}{E}\left[(1+\nu) \sigma_{11}-\nu I_{1}\right] \\
\epsilon_{22}=\frac{1}{E}\left[(1+\nu) \sigma_{22}-\nu I_{1}\right] \\
\epsilon_{33}=\frac{1}{E}\left[(1+\nu) \sigma_{33}-\nu I_{1}\right] \\
\epsilon_{12}=\frac{1}{2 G} \sigma_{12} \\
\epsilon_{13}=\frac{1}{2 G} \sigma_{13} \\
\epsilon_{23}=\frac{1}{2 G} \sigma_{23}
\end{array}
$$

na forma inversa de (2.45):

$$
\begin{array}{r}
\sigma_{11}=\frac{E}{(1+\nu)(1-2 \nu)}\left[(1-2 \nu) \epsilon_{11}+\nu I_{1}\right] \\
\sigma_{22}=\frac{E}{(1+\nu)(1-2 \nu)}\left[(1-2 \nu) \epsilon_{22}+\nu I_{1}\right] \\
\sigma_{33}=\frac{E}{(1+\nu)(1-2 \nu)}\left[(1-2 \nu) \epsilon_{33}+\nu I_{1}\right] \\
\sigma_{12}=2 G \epsilon_{12} \\
\sigma_{13}=2 G \epsilon_{13} \\
\sigma_{23}=2 G \epsilon_{23}
\end{array}
$$

em notação indicial, pode-se escrever as equações (2.45) e (2.46):

$$
\epsilon_{i j}=\frac{1+\nu}{E} \sigma_{i j}-\frac{\nu}{E} I_{1} \delta_{i j}
$$




$$
\sigma_{i j}=\frac{E}{1+\nu} \epsilon_{i j}+\frac{\nu E}{(1+\nu)(1-2 \nu)} I_{1} \delta_{i j}
$$

com $i, j=1,2,3$. Podendo-se ainda escrever as relações:

$$
\lambda=\frac{2 G \nu}{(1-2 \nu)} \quad G=\frac{E}{2(1+\nu)}
$$

onde $\lambda$ e $G$ são conhecidos como constantes elásticas de Lamé. Com (2.49) pode-se escrever a equação (2.48) na forma:

$$
\sigma_{i j}=2 G \epsilon_{i j}+\lambda \delta_{i j} e
$$

onde $\delta_{\alpha \beta}$, é o delta de Kronecker, que apresenta valor nulo para $\alpha \neq \beta$ e valor unitário para $\alpha=\beta$ e $e=\epsilon_{11}+\epsilon_{22}+\epsilon_{33}=I_{1}$ representa o vetor de deformação cúbica.

\subsubsection{Equação de Navier}

Como foi visto nos ítens anteriores, o problema elástico é constituído por 15 incógnitas e para isto são escritas 15 equações:

$$
\begin{aligned}
\epsilon_{i j} & =\frac{1}{2}\left(u_{i, j}+u_{j, i}\right) & \text { relação deformação-deslocamento } \\
\sigma_{j i, j}+f_{i} & =0 & \text { equação de equilíbrio } \\
\sigma_{i j} & =\lambda \delta_{i j} \epsilon_{k k}+2 G \epsilon_{i j} & \text { relação tensão-deformação }
\end{aligned}
$$


Substituindo-se a equação (2.53), na expressão (2.52) e esta na equação (2.51), tem-se uma única equação que leva em conta as relações tensão-deformação, as relações de equilíbrio e a relação defomação-deslocamento.

$$
G u_{i, j j}+(G+\lambda) u_{j, j i}+f_{i}=0
$$

ou vetorialmente:

$$
G \nabla^{2} \boldsymbol{u}+(G+\lambda) \nabla(\nabla \cdot \boldsymbol{u})+\boldsymbol{f}=\mathbf{0}
$$

A expressão (2.55) é chamada de Equação de Navier, e transforma as 15 equações da elasticidade em 3 equações diferenciais de segunda ordem, havendo 3 incógnitas desconhecidas que são os deslocamentos $u_{i}$ onde $i=1,2,3$ e o vetor $\boldsymbol{f}$ são as forças de volume atuantes no sólido.

\subsubsection{Elasticidade Plana - 2D}

Existem alguns casos onde as componentes de tensão e deformação em certa direção, possuem valores desprezíveis se comparadas com valores em outras direções. Neste caso, podem-se desprezar as variáveis nesta direção, para que o sistema de equações de equilíbrio se tornem mais estáveis. Neste trabalho serão vistos o Estado Plano de Deformação e o Estado Plano de Tensões, onde a análise 3D será transformada em 2D.

\subsubsection{Estado Plano de Deformação}

Considere-se a figura (2.9), tendo o sólido a dimensão na direção $x_{3}$ muito maior que nas outras duas direções e solicitado por ações nas direções $x_{1}$ e $x_{2}$, que sejam constantes em relação ao eixo $x_{3}$. Retirando-se uma faixa de espessura unitária da estrutura da figura (2.9), pode-se dizer que na direção do eixo $x_{3}$ não há 


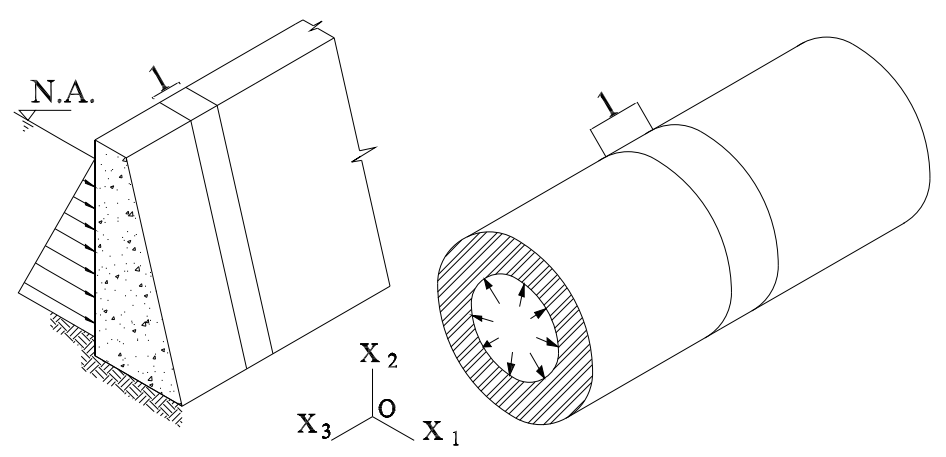

Fig. 2.9: Exemplos de Estado Plano de Deformação

deslocamentos, pois esta fatia do sólido está fortemente contida na estrutura nesta direção. Com isto, podem-se escrever as seguintes equações de deslocamentos:

$$
\begin{array}{r}
u_{i}=u_{i}\left(x_{1}, x_{2}\right) ; i=1,2 \\
u_{3}=0
\end{array}
$$

Substituindo-se a equação (2.56), na equação (2.37) da elasticidade 3D, tem-se:

$$
\begin{aligned}
\epsilon_{11} & =u_{1,1} & \epsilon_{22} & =u_{2,2} \\
\epsilon_{12} & =\frac{1}{2}\left(u_{1,2}+u_{2,1}\right) & \epsilon_{23} & =\frac{1}{2}\left(u_{2,3}+u_{3,2}\right)=0 \\
\epsilon_{13} & =\frac{1}{2}\left(u_{1,3}+u_{3,1}\right)=0 & \epsilon_{33} & =u_{3,3}=0
\end{aligned}
$$

ou indicialmente:

$$
\begin{array}{r}
\epsilon_{i j}=\frac{1}{2}\left(u_{i, j}+u_{j, i}\right) ; \quad i, j=1,2 \\
\epsilon_{i 3}=0
\end{array}
$$


Substituindo-se a relação da equação (2.58) na relação constitutiva 3D da equação (2.50), tem-se:

$$
\begin{array}{ll}
\sigma_{11}=2 G \epsilon_{11}+\lambda\left(\epsilon_{11}+\epsilon_{22}\right) & \sigma_{12}=2 G \epsilon_{12} \\
\sigma_{22}=2 G \epsilon_{22}+\lambda\left(\epsilon_{11}+\epsilon_{22}\right) & \sigma_{23}=0 \\
\sigma_{33}=\lambda\left(\epsilon_{11}+\epsilon_{22}\right) & \sigma_{13}=0
\end{array}
$$

ou indicialmente:

$$
\begin{array}{r}
\sigma_{i j}=2 G \epsilon_{i j}+\lambda \delta_{i j} \epsilon_{k k} ; \quad i, j, k=1,2 \\
\sigma_{i 3}=0 \\
\sigma_{33}=\lambda \epsilon_{k k}
\end{array}
$$

Nota-se, na expressão (2.59), que a tensão normal na direção $x_{3}$ é dependente apenas das deformações lineares nas outras direções. Substituindo a equação (2.60), nas equações de equilíbrio 3D (2.53), tem-se:

$$
\begin{aligned}
& \sigma_{11,1}+\sigma_{12,2}+b_{1}=0 \\
& \sigma_{12,1}+\sigma_{22,2}+b_{2}=0
\end{aligned}
$$

podendo ser escrito indicialmente:

$$
\sigma_{j i, j}+f_{i}=0 ; \quad i, j=1,2
$$


A equação de Navier para o estado plano de deformação pode ser escrita como:

$$
G u_{i, j j}+(G+\lambda) u_{j, j i}+f_{i}=0 ; \quad i, j, k=1,2
$$

\subsubsection{Estado Plano de Tensão}

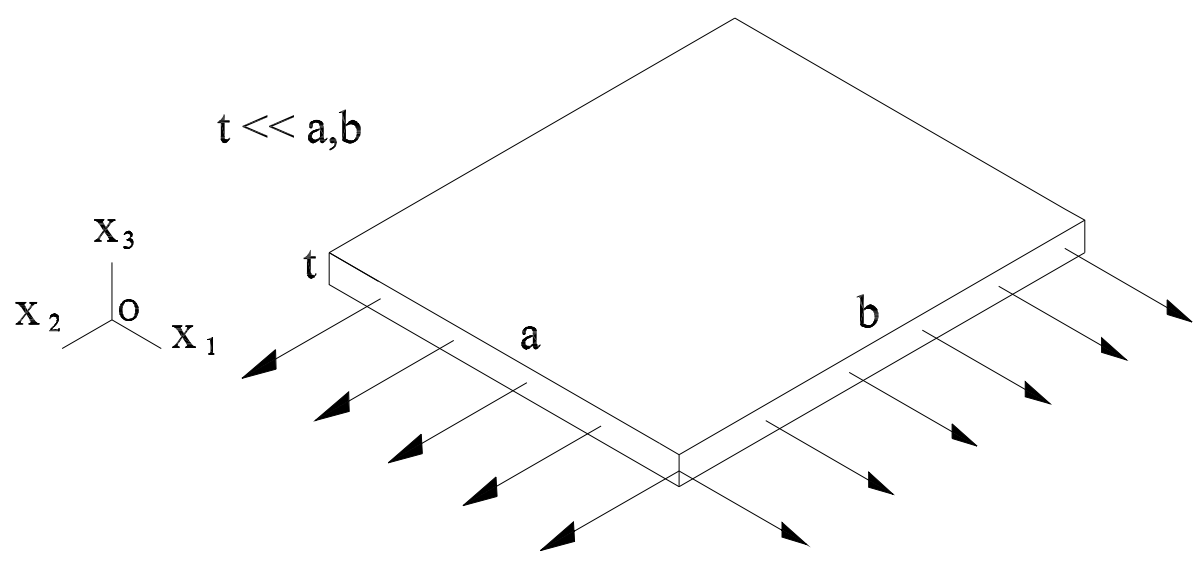

Fig. 2.10: Exemplo de Estado Plano de Tensão

Considere-se um sólido em que uma dimensão, denominada de espessura, é muito menor que as outras duas, figura (2.10), e que este corpo seja carregado com forças distribuídas normais uniformes a esta espessura. Admite-se que na direção $x_{3}$ da figura (2.10), as tensões sejam nulas, tem-se então:

$$
\sigma_{i 3}=0
$$

Admitindo a hipótese da equação (2.64), e utilizando-se da equação (2.50), tem-se: 


$$
\begin{array}{r}
\sigma_{33}=0=\lambda\left(\epsilon_{11}+\epsilon_{22}+\epsilon_{33}\right)+2 G \epsilon_{33} \\
\epsilon_{33}=-\frac{\lambda}{(\lambda+2 G)}\left(\epsilon_{11}+\epsilon_{22}\right)=-\frac{\lambda}{(\lambda+2 G)} e_{k k} ; \quad k=1,2
\end{array}
$$

Na expressão (2.65), observa-se que existe deformação axial na direção $x_{3}$, figura (2.10), e que é dependente apenas das variáveis dos outros eixos ortogonais. Escrevendo-se a equação (2.50), para os outros termos das tensões normais e tangenciais com $i, j=1,2$ tem-se a seguinte relação:

$$
\sigma_{i j}=\delta_{i j} \frac{2 \lambda G}{\lambda+2 G} \epsilon_{k k}+2 G \epsilon_{i j} ; \quad i, j, k=1,2
$$

A equação de Navier pode ser escrita com a expressão (2.66) como:

$$
G\left(\frac{3 \lambda+2 G}{\lambda+2 G}\right) u_{k, k i}+G u_{i, j j}+f_{i}=0 ; \quad i, j, k=1,2
$$




\section{MÉTODO DOS ELEMENTOS DE CONTORNO}

\subsection{Introdução}

Neste capítulo serão discutidas as particularidades do método numérico adotado, o Método dos Elementos de Contorno. Primeiramente serão mostradas as bases do método que são as soluções fundamentais, onde será discutida a origem de tais soluções.

Num segundo momento, será mostrado o próprio desenvolvimento do método, as equações de deslocamentos, de forças de superfície e de tensão no sólido, as técnicas de aproximação das variáveis no contorno e a montagem de equações algébricas, que podem ser resolvidas por métodos de resolução de sistemas lineares.

Por último se discutirá os métodos de integração das equações do método, onde foram adotadas integrais analíticas para todos os elementos e não só nos elementos singulares. Também será vista algumas técnicas de divisão dos elementos, que visam resolver problemas de quase singularidade, ou seja, em situações onde o ponto fonte está muito próximo do elemento integrado. As técnicas discutidas neste trabalho são os de subelementação, com divisão constante do elemento, e de divisão progressiva, onde a divisão do elemento se acentua nas proximidades do ponto fonte.

\subsection{Vetor de Galerkin}

Em 1930 Galerkin publicou um artigo, onde introduzia uma função que substituía os deslocamentos por funções derivadas de segunda ordem. Tal função era um vetor e, por isso, ficou conhecida como vetor de Galerkin. Nessa expressão os deslocamentos $u_{i}$ são substituídos pela expressão:

$$
2 G u_{i}=c g_{i, j j}-g_{j, j i}
$$


onde $c$ é uma constante, $g$ o próprio vetor de Galerkin e $G$ o módulo de elasticidade transversal. Substituindo-se a expressão (3.1) na equação de Navier (2.54), lembrando-se que os termos $\lambda$ e $(\lambda+G)$, podem ser escritos por:

$$
\lambda=\frac{2 \nu G}{(1-2 \nu)} \quad(\lambda+G)=\frac{G}{(1-2 \nu)}
$$

tem-se a seguinte expressão da equação de Navier escrita com os termos do vetor de Galerkin:

$$
\left(\frac{c}{2} g_{i, k k j j}-\frac{1}{2} g_{k, k i j j}\right)+\frac{1}{(1-2 \nu)}\left(\frac{c}{2} g_{j, k k j i}-\frac{1}{2} g_{k, k j j i}\right)+f_{i}=0
$$

Os termos derivativos $g_{k, k i j j}, g_{j, k k j i}$ e $g_{k, k j j i}$ são iguais, podendo-se então simplificar a expressão (3.3) escrevendo apenas a expressão:

$$
\frac{c}{2} g_{i, k k j j}+\left(-\frac{1}{2}+\frac{c}{2(1-2 \nu)}-\frac{1}{2(1-2 \nu)}\right) g_{k, k j j i}+f_{i}=0
$$

Uma solução particular para se encontrar a constante $c$ do vetor de Galerkin, seria de se fazer o termo $g_{k, k j j i}$ desaparecer, pois para o caso da inexistência de forças de volume, a expressão (3.4) se tornaria uma operador biarmônico ou uma equação de Laplace, $\nabla^{2}\left(\nabla^{2} \boldsymbol{g}\right)=\mathbf{0}$, então:

$$
-\frac{1}{2}+\frac{c}{2(1-2 \nu)}-\frac{1}{2(1-2 \nu)}=0 \Rightarrow c=2(1-\nu)
$$

O vetor de Galerkin (3.1), pode então ser escrito como: 


$$
2 \mu u_{i}=2(1-\nu) g_{i, j j}-g_{j, j i}
$$

onde a equação (3.4), fica escrita vetorialmente como:

$$
\nabla^{2}\left(\nabla^{2} \boldsymbol{g}\right)+\frac{1}{(1-\nu)} \boldsymbol{f}=\mathbf{0}
$$

com isto a equação (2.53), que se refere a relação tensão-deformação, pode ser expressa em função do vetor de Galerkin como:

$$
\sigma_{i j}=(1-\nu)\left[g_{i, k k j}+g_{j, k k i}\right]-g_{k, k i j}+\nu \delta_{i j} g_{l, l k k}
$$

onde, para o caso $2 \mathrm{D}, i, j, k, l=1,2$.

As componentes de deslocamento e das equações de equilíbrio podem então serem escritas com os termos do vetor de Galerkin.

$$
(1-\nu) g_{i, j j k k}+f_{i}=0
$$

onde $i, j, k=1,2$.

\subsection{Problemas de Kelvin em Elasticidade Plana}

O problema de Kelvin é se conhecer a resposta em um meio elástico infinito, neste caso em elasticidade plana, causada por uma força concentrada.

Seja um corpo sujeito a uma força pontual $P$, atuando em um corpo cilíndrico de raio $R$. Em se tratando de elasticidade plana, a força $P$ se torna uma carga 
distribuída constante ao longo da espessura do sólido, ou seja, uma carga distribuída por unidade da espessura do sólido. A geometria do sólido pode ser qualquer, mas neste caso foi utilizado um corpo cilíndrico, por ser extremamente simples se encontrar a normal a superfície $\left(n_{i}=x_{i} / R\right)$ no contorno deste sólido.

As condições de equilíbrio estático requerem que as reações de forças de superfície atuantes no corpo estejam em equilíbrio com a força aplicada $P$, como mostra a figura (3.1).

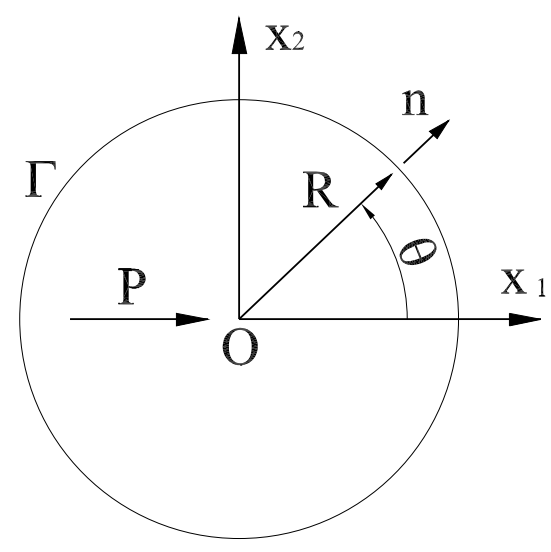

Fig. 3.1: Força concentrata em região plana cilíndrica

para uma força atuando na direção $x_{1}$, tem-se a seguinte equação de equilíbrio.

$$
\Sigma F_{1}=0=P+\int_{\Gamma} t_{1} d \Gamma=P+\int_{\Gamma} \sigma_{j 1} n_{j} d \Gamma=\int_{\Gamma} \sigma_{j 1} \frac{x_{j}}{R} d \Gamma+P
$$

onde $t_{1}$ é a reação, em termos de forças de superfície, distribuída em todo o contorno $\Gamma$ do corpo cilíndrico, à força $P$. O termo $\sigma_{j 1} n_{j}$ é equivalente a força de superfície no contorno $\Gamma$ segundo a relação de Cauchy sendo $n_{j}$ a normal ao contorno $\Gamma$.

Substituindo-se o termo de tensão $\sigma_{j 1}$ da equação (3.10)por (3.8) tem-se o termo (3.10) escrito em função do vetor de Galerkin:

$$
P+\int_{\Gamma} \frac{x_{j}}{R}\left\{(1-\nu)\left[g_{1, k k j}+g_{j, k k 1}\right]-g_{k, k 1 j}+\nu \delta_{1 j} g_{1,1 k k}\right\} d \Gamma=0
$$


Para integrar os termos da equação (3.11), o elemento infinitesimal do contorno $d \Gamma$ pode ser escrito por coordenadas cilíndricas $R d \theta$. Para se solucionar a equação (3.11), é preciso adotar soluções particulares para $g_{i}$, onde $i=1,2$. Adotando-se as seguintes soluções particulares:

$$
g_{1}=b R^{2} \ln R ; \quad g_{2}=0
$$

substituindo-se tais soluções nas expressões do vetor de Galerkin e derivando os termos, tem-se:

$$
\begin{array}{r}
\frac{1}{b} g_{1, i}=x_{i}(2 \ln R+1) \\
\frac{1}{b} g_{1, i j}=\delta_{i j}(2 \ln R+1)+2 R^{-2} x_{i} x_{j} \\
\frac{1}{b} g_{1, i j k}=2 R^{-2}\left\{x_{i} \delta_{j k}+x_{j} \delta_{i j}-2 R^{-2} x_{i} x_{j} x_{k}\right\} \\
\frac{1}{b} g_{1, i i j}=4 R^{-2} x_{j}
\end{array}
$$

Introduzindo as equações (3.15) e (3.16) na equação (3.11), tem-se:

$$
-P=b \int_{\Gamma}\{4(1-\nu)-2\} R^{-1} d \Gamma+b \int_{\Gamma}\{4(1-\nu)+4 \nu\} x_{1}^{2} R^{-3} d \Gamma
$$

Como na equação (3.17), os termos das integrais estão escritas com a variável do raio $R$, então, por facilidade de integração, tal expressão pode ser escrita em coordenadas cilíndricas, onde o domínio de integração varia de 0 a $2 \pi$, tendo-se o ângulo: 


$$
\frac{x_{1}}{R}=\cos \theta
$$

Substituindo-se os limites de integração e o ângulo da equação (3.18) na equação (3.17), e integrando-se em todo o contorno da figura (3.1),tem-se:

$$
-P=\pi b\{4(1-2 \nu)+4\}=8 \pi b(1-\nu)
$$

podendo-se então encontrar o valor da constante $b$ da equação (3.12):

$$
b=\frac{-P}{8 \pi(1-\nu)}
$$

A constante $b$, da equação (3.20), satisfaz as equações de equilíbrio estático do cilindro, independentemente das suas dimensões, e as equações de Navier da elasticidade plana, ao mesmo tempo.

\subsubsection{Equação fundamental de deslocamento}

Considerando-se a figura (3.1), a força pontual na direção $x_{1}$ pode ser aplicada sob a forma de Delta de Dirac, isto é, o carregamento neste caso só tem valor na direção $x_{1}$ e no ponto de aplicação da força. A equação de deslocamento de Kelvin, utilizando as funções do vetor de Galerkin e a função de Delta de Dirac, pode ser escrita como:

$$
2 G u_{i}=2(1-\nu) g_{i, j j}-g_{j, j i}=2(1-\nu) \delta_{i 1} g_{1, j j}-g_{1,1 i}
$$


A função de Delta de Dirac, é matematicamente equivalente ao efeito de uma força pontual aplicada em um ponto $\xi$. A propriedade da função Delta de Dirac é que, para qualquer ponto em $x$, a função é zero e na vizinhança do ponto $\xi$, num valor limite onde $\epsilon \rightarrow 0$, o valor tende abruptamente ao infinito, como mostra a equação (3.22) e a figura (3.2)

$$
\int_{-\infty}^{\infty} \delta(x, \xi) d x=1(\xi)
$$

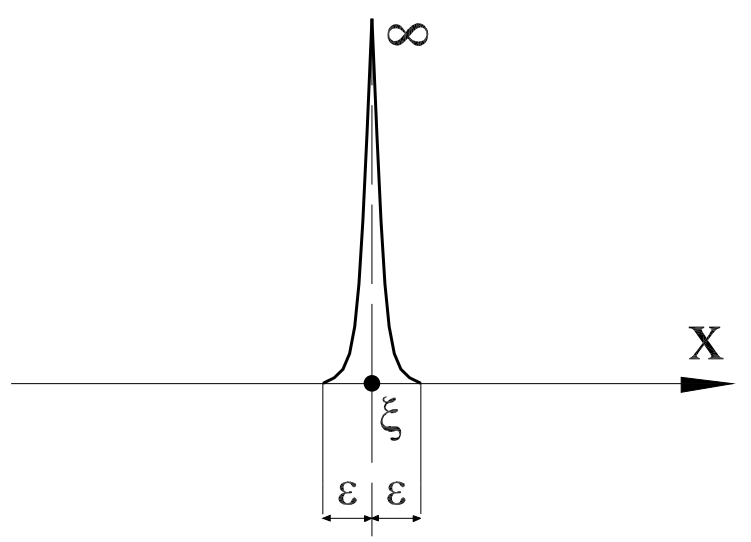

Fig. 3.2: Função Delta de Dirac

Em se tratando de elasticidade plana, deseja-se saber também a resposta em termos de deslocamentos de uma ação aplicada na direção $x_{2}$. Basta então escrever uma equação genérica para todas as direções, bastando substituir o índice da direção 1 por um índice genérico $k$ na equação (3.21).

$$
2 G u_{i k}=2(1-\nu) \delta_{i k} g_{k, j j}-g_{k, k i}
$$

Com a introdução do índice $k$ na equação (3.23), pode-se interpretar fisicamente tal equação, como sendo a resposta em termo de deslocamentos na direção $i$, causado por uma força unitária aplicada na direção $k$. 
Com os termos do vetor de Galerkin da equação (3.23), modificados pela introdução do índice $k$, tem-se na equação (3.14):

$$
\begin{array}{r}
\left.g_{k, j j}=\delta_{j j}(2 \ln R+1)+2 R^{-2} x_{j} x_{j}=2(2 \ln R+1)+2 R^{-2} R^{2}=4 \ln R+43.24\right) \\
g_{k, k i}=\delta_{k i}(2 \ln R+1)+2 R^{-2} x_{k} x_{i}=\delta_{k i}(2 \ln R+1)+2 R,,_{k} R, i(3.25)
\end{array}
$$

onde os termos $R, i$, são os cossenos diretores e dependendo dos índices, os quais podem variar de 1 a 2 , são expressos por:

$$
R,_{1}=\cos (\theta) \quad R, 2_{2}=\operatorname{sen}(\theta)
$$

Substituindo-se as equações (3.24) e (3.25) na equação (3.23), tem-se:

$$
\begin{array}{r}
u_{i k}=\frac{b}{2 G}\left\{2(1-\nu) \delta_{i k}(4 \ln R+4)+\delta_{k i}(\ln R+1)-2 R,_{k} R,_{i}\right\} \\
u_{i k}=\frac{-2 P}{2 G 8 \pi(1-\nu)}\left\{\delta_{i k}[4(1-\nu)-1] \ln R+\delta_{i k}\left[4-\frac{1}{2}\right]-R_{, k} R,,_{i}\right\} \\
u_{i k}=\frac{-P}{8 G \pi(1-\nu)}\left\{\left[(3-4 \nu) \ln R-\frac{7}{2}\right] \delta_{i k}+R_{, i} R,,_{k}\right\}
\end{array}
$$

frequentemente, em muitas referências, o termo 7/2 é omitido, (Kane, 1994).

\subsubsection{Equação Fundamental de Tensão}

A equação (3.8), escrita em função do vetor de Galerkin para problemas de elasticidade plana, pode ser escrita em função de força pontual aplicada na direção 1, utilizando-se a função de delta de Dirac:

$$
\sigma_{i j}=b\left\{(1-\nu)\left[\delta_{i 1} g_{1,11 j}+\delta_{j 1} g_{1,11 i}\right]-g_{1,1 i j}+\nu \delta_{i j} g_{1, k 11}\right\}
$$


Trocando-se o índice 1 pelo índice livre $k$, pode-se escrever as tensões $\sigma_{i j}$ nas direções $i, j=1,2$, causadas por uma força pontual aplicada na direção $k$, onde $k$ pode assumir as direções 1 e 2 .

$$
\sigma_{i j k}=b\left\{(1-\nu)\left[\delta_{i k} g_{k}, k k j+\delta_{j k} g_{k, k k i}\right]-g_{k, k i j}+\nu \delta_{i j} g_{k, k k k}\right\}
$$

Substituindo-se as equações (3.15) e (3.16) na equação (3.29), tem-se:

$$
\sigma_{i j k}=\frac{-P(1-2 \nu)}{4 \pi(1-\nu) R}\left\{R,,_{i} \delta_{j k}+R,,_{j} \delta_{k i}-R,,_{k} \delta_{i j}+\frac{2}{(1-2 \nu)} R,_{i} R,,_{j} R,,_{k}\right\}
$$

\subsubsection{Equação Fundamental de Forças de Superfície}

Utilizando-se a expressão (3.30), pode-se obter o vetor das forças de superfície bastando utilizar as relações de Cauchy.

$$
\begin{array}{r}
p_{i j}=\sigma_{i j k} n_{j}=\frac{-P(1-2 \nu)}{4 \pi(1-\nu) R}\left\{R,{ }_{i} \delta_{j k} n_{j}+R,{ }_{j} \delta_{k i} n_{j}-R,{ }_{k} \delta_{i j} n_{j}+\right. \\
\left.+\frac{2}{(1-2 \nu)} R,{ }_{i} R,{ }_{j} R,,_{k} n_{j}\right\} \\
p_{i j}=\frac{-P}{4 \pi(1-\nu) R}\left\{(1-2 \nu)\left[R,{ }_{i} n_{j}-R,{ }_{j} n_{i}\right]+R,{ }_{j} n_{j}\left[(1-2 \nu) \delta_{j i}+2 R,{ }_{i} R,,_{j}\right]\right\}
\end{array}
$$

onde $i, j=1,2$ e o termo $n$ é a normal à superfície onde está atuando a força de superfície (3.31). 


\subsection{Formulação Direta de Elemento de Contorno}

A formulação direta do Método dos Elementos de Contorno é baseada no teorema da reciprocidade dos trabalhos virtuais, onde as forças e deslocamentos em um sistema real de um corpo delimitado por um contorno $\Gamma$ e possuidor de um área $\Omega$, estão em equilíbrio com as forças e deslocamentos aplicados em um corpo com sistema de coordenadas * o qual tende ao infinito, como mostra a figura (3.3). Considera-se neste caso o material elástico linear, isotrópico, e sendo o problema de elasticidade plana.

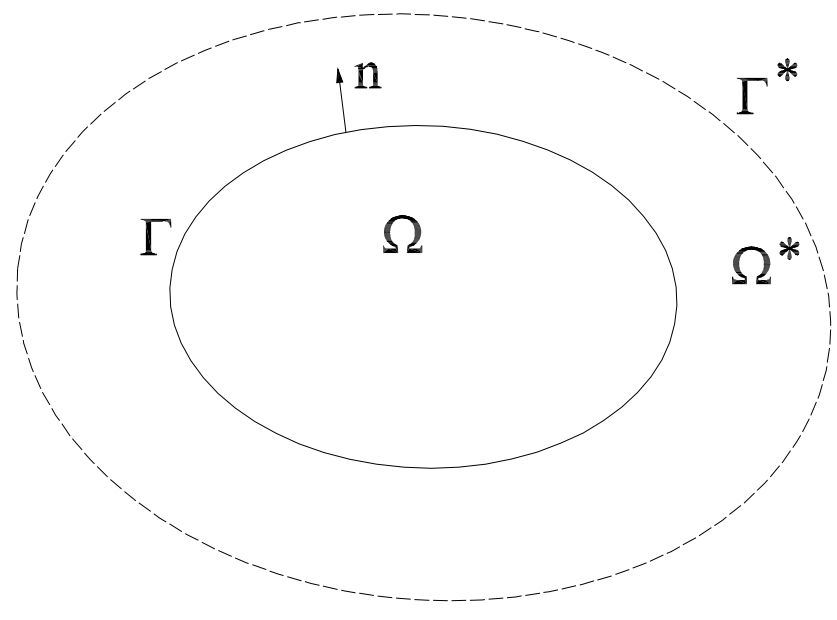

Fig. 3.3: Sólido de área $\Omega$ e contorno $\Gamma$ contido num sólido de dimensões infinitas *

A relação de trabalhos virtuais de forças aplicadas em um sistema real, com resposta em termos de forças e deslocamentos em um sistema de coordenadas infinitas * e vice versa, pode ser escrito por:

$$
\int_{\Gamma} p_{i}^{*} u_{i}(x) d \Gamma(x)+\int_{\Omega} \psi_{i}^{*} u_{i}(z) d \Omega(z)=\int_{\Gamma} p_{i}(x) u_{i}^{*} d \Gamma(x)+\int_{\Omega} \psi_{i}(z) u_{i}^{*}(z) d \Omega(z)
$$

onde $x$ é um ponto no contorno $\Gamma$ e $z$ é um ponto no domínio $\Omega$. Os termos $u_{i}$ e $t_{i}$ são os deslocamentos e forças de superfície no contorno $\Gamma$, respectivamente. Os termos escritos com o índice $*$ se referem ao domínio de contorno infinito $\Gamma^{*}$. Os 
termos $\psi$ se referem às forças de volume atuando tanto no domínio real quanto no infinito $\Omega^{*}$.

Substituindo-se os termos das coordenadas $*$, por termos escritos em função de solução fundamental para deslocamentos, forças de superfície ou forças de volume para um ponto $x$ ou $z$ do contorno ou do domínio do sólido, respectivamente, sendo causadas pela aplicação de uma força pontual unitária no ponto $\xi$, tem-se:

$$
\begin{aligned}
\int_{\Gamma} H_{i j}^{*}(x, \xi) u_{i}(x) d \Gamma(x)+\int_{\Omega} \delta_{i j} \delta(z, \xi) u_{i}(z) d \Omega(z)= & \int_{\Gamma} p_{i}(x) G_{i j}^{*}(x, \xi) d \Gamma(x)+ \\
& +\int_{\Omega} \psi_{i}(z) G_{i j}^{*}(z, \xi) d \Omega(z)
\end{aligned}
$$

onde o termo $\delta_{i j} \delta(z, \xi)$, no segundo termo a esquerda da expressão (3.33), que é uma função de Delta de Dirac, substitui a equação de solução fundamental $\psi_{i j}^{*}$, a qual corresponde respostas em termos de forças de volume na direção $i$, causadas por uma força unitária aplicada na direção $j$. Com a utilização desta função a resposta, como mostra a expressão (4.6), vai ser unitária na direção $i$. Com isto, a integral do segundo termo à esquerda da equação (3.33), pode ser escrita por:

$$
\int_{\Omega} \delta_{i j} \delta(z, \xi) u_{i}(z) d \Omega(z)=\int_{\Omega} u_{j}(z) \delta(z, \xi) d \Omega(z)=c u_{j}(\xi)
$$

onde $c=1$, para pontos dentro do domínio $\Omega$ e $c=0$, para os ponto fora do contorno $\Gamma$. Então substituindo a expressão (3.34) no segundo termo da equação (3.33), pode-se obter:

$$
u_{i}(\xi)=\int_{\Gamma}\left[G_{i j}^{*}(x, \xi) p_{j}(x)-H_{i j}^{*}(x, \xi) u_{j}(x)\right] d \Gamma(x)+\int_{\Omega} G_{i j}^{*}(z, \xi) \psi_{j}(z) d \Omega(z)
$$

A equação (3.35) é a equação para deslocamento, nas direções de $i=1,2$ do ponto fonte $\xi$, o qual é um ponto interno ao domínio $\Omega$. Tal ponto se relaciona com 
deslocamentos, forças de superfície e forças de domínio, as quais estão escritas nos pontos campo do contorno $x$, ou do domínio $z$, na direção de $j=1$, 2, e também com a soluções fundamentais $H_{i j}^{*}$ e $G_{i j}^{*}$ de forças de superfície e de deslocamentos, respectivamente, também escritas para pontos do contorno. Esta equação é conhecida como identidade de Somigliana para deslocamentos.

Escrevendo-se a equação (3.35) para pontos do contorno, ocorrerão problemas de singularidade nas integrais da referida equação. Quando o ponto fonte coincidir com o ponto campo, o raio tenderá a zero $\xi \rightarrow \xi_{0}$. As soluções fundamentais $H_{i j}^{*}$ e $G_{i j}^{*}$ possuem termos os quais podem causar problemas de integração, que são $\ln R$ e $1 / R$, respectivamente.

Seja o domínio com contorno $\Gamma$, seccionado e acrescentado de um domínio semi-circular $\Gamma_{\epsilon}$, o qual possui um raio $R$ que tende a zero, como mostra a figura $(3.4)$.

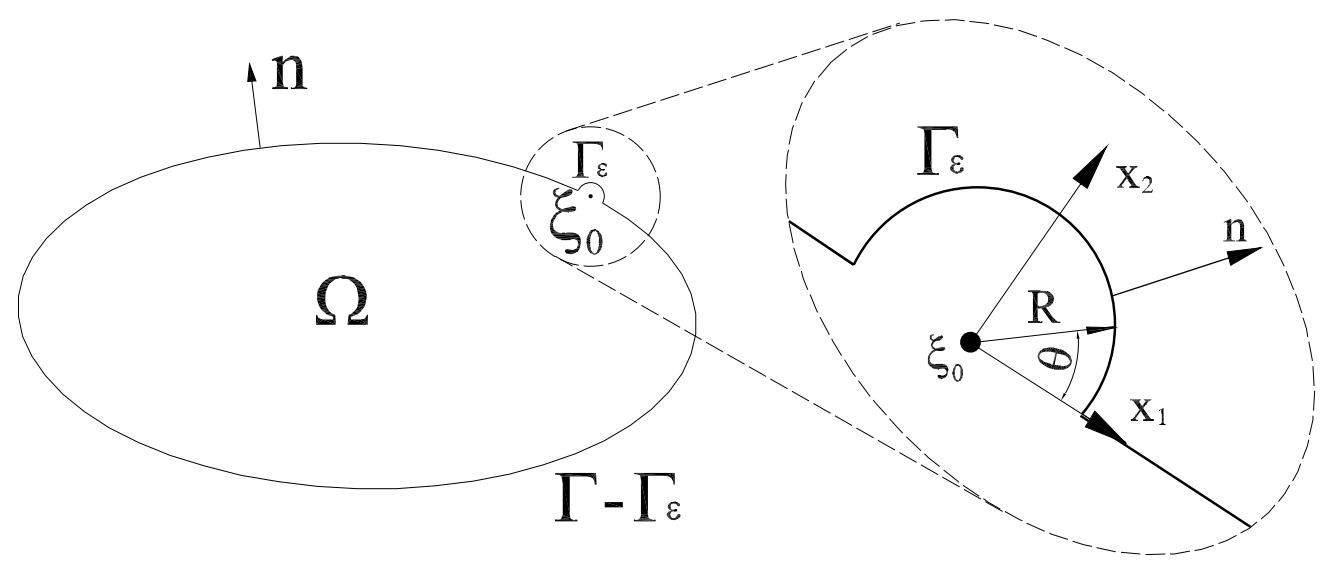

Fig. 3.4: Contorno adicional com raio tendendo a zero

Escrevendo as equações das integrais de contorno, primeiramente para os termos de contorno contendo as soluções fundamentais de deslocamentos, tem-se:

$$
I_{i k}^{*}=\int_{\Gamma} G_{i k}^{*} p_{i} d \Gamma=\lim _{R \rightarrow 0}\left\{\int_{\Gamma-\Gamma_{\epsilon}} G_{i k}^{*} p_{i} d \Gamma+\int_{\Gamma_{\epsilon}} G_{i k}^{*} p_{i} d \Gamma\right\}=I_{i k}^{(1) *}+I_{i k}^{(2) *}
$$

O primeiro termo da equação (3.36) $I_{k}^{(1) *}$ não possui singularidade e pode ser 
integrado normalmente. No segundo termo, integra-se segundo o eixo local, como mostra a figura (3.4).

$$
I_{i k}^{(2) *}=\int_{\Gamma_{\epsilon}}-\frac{1}{8 G \pi(1-\nu)}\left\{[(3-4 \nu) \ln R] \delta_{i k}+R,,_{i} R, k\right\} d \Gamma_{\epsilon}
$$

Escrevendo-se os termos da equação (3.37) em coordenadas cilíndricas, tem-se os seguintes termos:

$$
R,_{1}=\cos \theta ; \quad R,_{2}=\operatorname{sen} \theta ; \quad d \Gamma_{\epsilon}=R d \theta
$$

lembrando-se que neste caso, para contornos suaves, o limite de integração, que é o ângulo $\theta$, varia de $\pi$ a 0 . Pode-se então integrar a equação (3.37) em coordenadas cilíndricas.

$$
I_{i j}^{(2) *}=\lim _{R \rightarrow 0}\left\{\int_{0}^{\pi}-\frac{1}{8 G \pi(1-\nu)}\left\{[(3-4 \nu) \ln R]+R,,_{i} R, k\right\} R d \theta\right\}=0
$$

Para os termos da solução fundamental de forças de superfície, tem-se a seguinte expressão para o caso do ponto fonte coincidir com o ponto campo.

$$
J_{i k}^{*}=\int_{\Gamma} H_{i k}^{*} u_{i} d \Gamma=\lim _{R \rightarrow 0}\left\{\int_{\Gamma-\Gamma_{\epsilon}} H_{i k}^{*} u_{i} d \Gamma+\int_{\Gamma_{\epsilon}} H_{i k}^{*} u_{i} d \Gamma\right\}=J_{i k}^{(1) *}+J_{i k}^{(2) *}
$$

O termo $J_{i k}^{(1) *}$ não possui singularidade e é integrado normalmente. Considerando os limites de integração para o caso de contornos suaves e, escrevendo todos os termos da equação fundamental $H_{i k}^{*}$ em coordenadas cilíndricas, tem-se para cada termo da solução fundamental $J_{i k}^{(2) *} \operatorname{com} i, j=1,2$ : 


$$
\begin{array}{r}
J_{11}^{(2) *}=\lim _{R \rightarrow 0}\left\{\int_{0}^{\pi} \frac{c_{1}}{R}\left\{(1-2 \nu)\left[\cos ^{2} \theta-\cos ^{2} \theta\right]+\left[(1-2 \nu)+2 \cos ^{2} \theta\right]\right\} R d \theta\right\} \\
=\lim _{R \rightarrow 0}\left\{\left.\frac{-1}{4 \pi(1-\nu)}\left\{\theta(1-2 \nu)+2\left[\frac{\theta}{2}+\frac{\operatorname{sen} 2 \theta}{4}\right]\right\}\right|_{0} ^{\pi}\right\}=-\frac{1}{2} \\
J_{12}^{(2) *}=J_{21}^{(2) *}=\lim _{R \rightarrow 0}\left\{\int_{0}^{\pi} \frac{c_{1}}{R}\{(1-2 \nu)[\cos \theta \operatorname{sen} \theta-\operatorname{sen} \theta \cos \theta]+\right. \\
+2 \cos \theta \operatorname{sen} \theta\} R d \theta\}=\lim _{R \rightarrow 0}\left\{\left.\frac{-1}{4 \pi(1-\nu)} \operatorname{sen}^{2} \theta\right|_{0} ^{\pi}\right\}=0 \\
J_{22}^{(2) *}=\lim _{R \rightarrow 0}\left\{\int_{0}^{\pi} \frac{c_{1}}{R}\left\{(1-2 \nu)\left[\operatorname{sen}^{2} \theta-\operatorname{sen}^{2} \theta\right]+\left[(1-2 \nu)+2 \operatorname{sen}^{2} \theta\right]\right\} R d \theta\right\} \\
=\lim _{R \rightarrow 0}\left\{\left.\frac{-1}{4 \pi(1-\nu)}\left\{\theta(1-2 \nu)+2\left[\frac{\theta}{2}-\frac{\operatorname{sen} 2 \theta}{4}\right]\right\}\right|_{0} ^{\pi}\right\}=-\frac{1}{2}
\end{array}
$$

onde:

$$
c_{1}=\frac{-1}{4 \pi(1-\nu)}
$$

Assim a equação para deslocamentos para pontos do contorno, considerando a posição do ponto fonte em contornos suaves, pode ser escrita como:

$$
c_{i j} u_{i}=\int_{\Gamma}\left[G_{i j}^{*} p_{i}-H_{i j}^{*} u_{i}\right] d \Gamma+\int_{\Omega} G_{i j}^{*} \psi_{i} d \Omega
$$

sendo $c_{i j}$, escrito matricialmente conforme equações (3.41):

$$
c_{i j}=-\left[\begin{array}{cc}
1 / 2 & 0 \\
0 & 1 / 2
\end{array}\right]
$$


A equação (3.44) somente é válida para contornos suaves, mas em contornos onde aparecem cantos, deve-se adotar novos limites de integração, como mostra a figura (3.5).

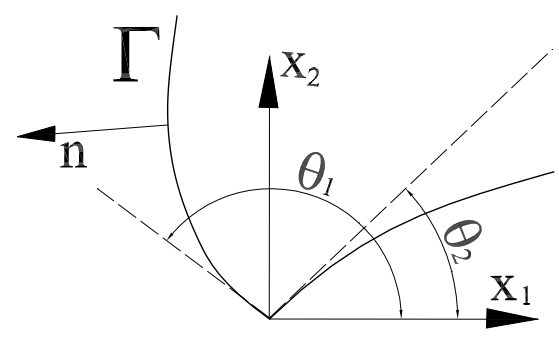

Fig. 3.5: Geometria de um contorno de canto anguloso

Sendo assim, com o novo limite de integração, o ângulo $\theta$ vai variar de $\theta_{1}$ até $\theta_{2}$. Introduzindo-se esses limites nas equações (3.41), tem-se:

$$
\begin{array}{r}
J_{11}^{(2) *}=\lim _{R \rightarrow 0}\left\{\int_{\theta_{1}}^{\theta_{2}} \frac{c_{1}}{R}\left\{(1-2 \nu)\left[\cos ^{2} \theta-\cos ^{2} \theta\right]+\left[(1-2 \nu)+2 \cos ^{2} \theta\right]\right\} R d \theta\right\} \\
=\lim _{R \rightarrow 0}\left\{\left.c_{1}\left\{\theta(1-2 \nu)+2\left[\frac{\theta}{2}+\frac{\operatorname{sen} 2 \theta}{4}\right]\right\}\right|_{\theta_{1}} ^{\theta_{2}}\right\}=2 c_{1}(1-\nu)\left(\theta_{2}-\theta_{1}\right)+ \\
+\frac{\operatorname{sen} 2 \theta_{2}-\operatorname{sen} 2 \theta_{1}}{2} \\
J_{12}^{(2) *}=J_{21}^{(2) *}=\lim _{R \rightarrow 0}\left\{\int_{\theta_{1}}^{\theta_{2}} \frac{c_{1}}{R}\{(1-2 \nu)[\cos \theta \operatorname{sen} \theta-\operatorname{sen} \theta \cos \theta]+\right. \\
J_{22}^{(2) *}=\lim _{R \rightarrow 0}\left\{\int_{\theta_{1}}^{\theta_{2}} \frac{c_{1}}{R}\left\{(1-2 \nu)\left[\operatorname{sen}^{2} \theta-\operatorname{sen}^{2} \theta\right]+[(1-2 \nu)+2 \cos \theta \operatorname{sen} \theta\} R d \theta\right\}\right. \\
=\lim _{R \rightarrow 0}\left\{\left.c_{1}\left\{\theta(1-2 \nu)+2\left[\frac{\theta}{2}-\frac{\operatorname{sen}^{2} \theta}{4}\right]\right\}\right|_{\theta_{1}} ^{\theta_{2}}\right\}=2 c_{1}(1-\nu)\left(\theta_{2}-\theta_{1}\right)+
\end{array}
$$


$+\frac{\operatorname{sen} 2 \theta_{2}-\operatorname{sen} 2 \theta_{1}}{2}$

onde $\left.c_{1}=-1 /(4 \pi-\nu)\right)$; matricialmente os termos $c_{i j}$ descritos nas equações (3.43) podem ser escrito como:

$$
c_{i j}=\left[\begin{array}{cc}
2(1-\nu)\left(\theta_{2}-\theta_{1}\right) \frac{\operatorname{sen} 2 \theta_{2}-\operatorname{sen} 2 \theta_{1}}{2} & \left(\operatorname{sen}^{2} \theta_{2}-\operatorname{sen}^{2} \theta_{1}\right) \\
\left(\operatorname{sen}^{2} \theta_{2}-\operatorname{sen}^{2} \theta_{1}\right) & 2(1-\nu)\left(\theta_{2}-\theta_{1}\right) \frac{\operatorname{sen} 2 \theta_{2}-\operatorname{sen} 2 \theta_{1}}{2}
\end{array}\right]
$$

Utilizando a equação (3.35) e a relação de compatibilidade de deslocamentos, tem-se a equação de deformações para pontos internos em função das variáveis do contorno.

$$
\epsilon_{i j}=\int_{\Gamma}\left[B_{i j k}^{*}(x, \xi) p_{k}(x)-T_{i j k}^{*}(x, \xi) u_{k}(x)\right] d \Gamma
$$

onde, segundo a relação da compatibilidade de deslocamentos.

$$
\begin{aligned}
& B_{i j k}^{*}=\frac{1}{2}\left(\frac{\partial G_{k i}^{*}}{\partial \xi_{j}}+\frac{\partial G_{k j}^{*}}{\partial \xi_{i}}\right) \\
& T_{i j k}^{*}=\frac{1}{2}\left(\frac{\partial H_{k i}^{*}}{\partial \xi_{j}}+\frac{\partial H_{k j}^{*}}{\partial \xi_{i}}\right)
\end{aligned}
$$

onde pode-se escrever os termos:

$$
B_{i j k}^{*}=\frac{1}{8 \pi G(1-\nu) R^{2}}\left[(1-2 \nu)\left(\delta_{i k} R,,_{j}+\delta_{j k} R,,_{i}\right)+2 R,,_{i} R,,_{j} R,,_{k}-\delta_{i j} R, k\right]
$$




$$
\begin{array}{r}
T_{i j k}^{*}=\frac{-1}{8 \pi(1-\nu) R^{2}}\left\{(1-2 \nu)\left(\delta_{j k}-2 R_{j} R_{k}\right) n_{i}-\left[(1-2 \nu) \delta_{i k}+\right.\right. \\
\left.+2 \nu R,_{i} R,_{k}\right] n_{j}-\left[(1-2 \nu) \delta_{i j}+2 R,_{i} R,,_{j}\right] n_{k}-2 \frac{\partial R}{\partial n}\left[\nu \delta_{i j} R,_{k}+\right. \\
\left.\left.+\nu \delta_{i k} R,,_{j}+\delta_{j k} R,,_{i}-4 R,_{i} R,,_{j} R,,_{k}\right]\right\}
\end{array}
$$

Utilizando a relação tensão-deformação da Lei de Hooke na equação (3.47), tem-se:

$$
\sigma_{i j}=\int_{\Gamma}\left[D_{i j k}^{*}(x, \xi) p_{k}(x)-S_{i j k}^{*}(x, \xi) u_{i}(x)\right] d \Gamma
$$

onde:

$$
\begin{gathered}
D_{i j k}^{*}=\frac{1}{4 \pi(1-\nu) R}\left[(1-2 \nu)\left(\delta_{k i} R,,_{j}+\delta_{k j} R,_{i}-\delta_{i j} R,,_{k}\right)+2 R,,_{i} R,_{j} R,,_{k}\right] \\
S_{i j k}^{*}=\frac{G}{2 \pi(1-\nu) R^{2}}\left\{2 \frac { \partial R } { \partial n } \left[(1-2 \nu) \delta_{i j} R,,_{k}+\nu\left(\delta_{i k} R,,_{j}+\delta_{j k} R,,_{i}\right)+\right.\right. \\
\left.-4 R,,_{i} R,{ }_{j} R,_{k}\right]+2 \nu\left(n_{i} R,,_{j} R,,_{k}+n_{j} R,,_{i} R,,_{k}\right)+(1-2 \nu) \\
\left.\left(2 n_{k} R,,_{i} R,,_{j}+n_{j} \delta_{i k}+n_{i} \delta_{j k}\right)-(1-4 \nu) n_{k} \delta_{i j}\right\}
\end{gathered}
$$

A representação das integrais de tensão no contorno é possível, como demonstra (Guiggiani, 1994), bastando para isso calcular o limite para um ponto $\xi$ do contorno, fazendo-o tender a zero. No entanto, as equações de tensão para o contorno só podem ser escritas em contornos suaves, pois as derivadas primeiras dos deslocamentos devem ter continuidade. 


\subsection{Formulação algébrica das equações de contorno}

A equação (3.43) descreve o comportamento físico de um sólido 2D e dificilmente será resolvida analiticamente, devido a complexidade geométrica, de vinculações e solicitações que os sólidos estão submetidos. Com a ajuda de técnicas computacionais e de métodos numéricos, pode-se dividir o sólido em elementos (neste caso elementos de contorno), onde as variáveis são aproximadas por funções aproximadoras, as quais podem ser de ordem qualquer, como mostra a figura (3.6), entretanto, são escritas normalmente na forma polinomial. Reunindo tais integrações de cada elemento, pode-se escrever uma equação algébrica para todos os nós dos elementos que, separando os termos incógnitos e prescritos do contorno, se transforma em um sistema linear, sendo este resolvido pelos vários processos computacionais existentes.

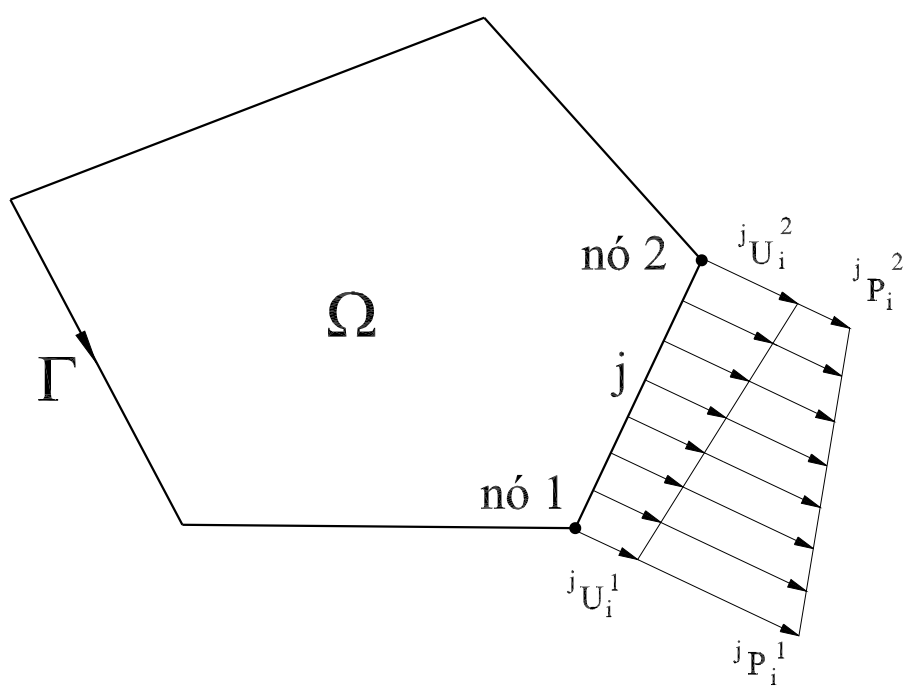

Fig. 3.6: Discretização do contorno - elementos retos e aproximação linear de $U$ e $P$

A expressão (3.43), pode ser escrita, desprezando-se as forças de domínio, para cada elemento de contorno como:

$$
c_{i j} U_{i}(d)=\sum_{E=1}^{N E} \int_{\Gamma_{E}}\left[G_{i j}^{*} \phi_{m}^{E}(t) P_{i}(x, \xi)-H_{i j}^{*} \phi_{m}^{E}(u) U_{i}(x, \xi)\right] d \Gamma_{E}
$$


onde $E$ se refere ao elemento, $N E$ é o número total de elementos de contorno da estrutura, $\phi_{m}^{E}$ é a função aproximadora que aproximará forças de superfície e deslocamentos sobre cada elemento e $\Gamma_{E}$ é o contorno do elemento.

A adoção de funções aproximadoras consiste em se utilizar uma função local parametrizada para cada elemento, que assuma valores unitários nos nós e valores maiores que 0 e menores que 1 no restante do elemento. É adotado um sistema de coordenadas onde o eixo local $\zeta$ coincide com o eixo do elemento. As funções aproximadoras são dadas por coordenadas normalizadas $\eta=\zeta / L$ para os nós do elemento, como mostra a figura (3.7):
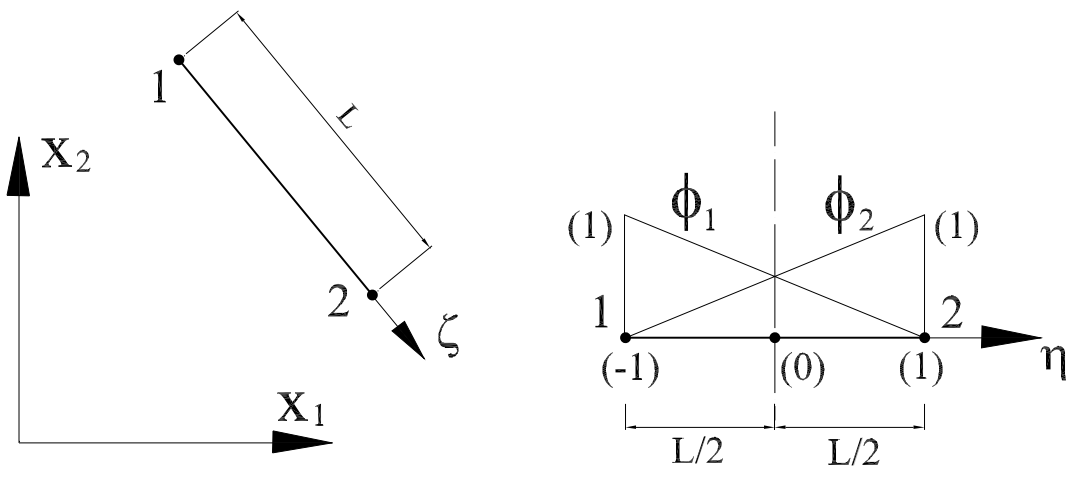

Fig. 3.7: Funções aproximadoras e coordenadas paramétricas

Nesse trabalho, foi adotado elemento com geometria linear para o contorno, com função aproximadora linear para forças de superfície e deslocamentos. Com isto, como mostra a figura (3.7), têm-se as seguintes funções aproximadoras para cada elemento:

$$
\begin{aligned}
\phi_{1} & =\frac{1}{2}(1+\eta) \\
\phi_{2} & =\frac{1}{2}(1-\eta)
\end{aligned}
$$

Escrevendo-se a equação (3.54) em uma forma matricial, tem-se: 


$$
H U-G P=0
$$

onde $\boldsymbol{H}$ é a matriz que contém todos os termos $H_{i j}^{*}$ integrados sobre o contorno, inclusive com as contribuições dos termos $c_{i j}$, e $\boldsymbol{G}$ é a matriz que contém todos os termos $G_{i j}^{*}$ integrados sobre contorno. $U$ e $P$ são os valores nodais no contorno de deslocamentos e forças de superfície, respectivamente. Considerando-se os valores prescritos no contorno, a equação (3.56) pode ser escrita na forma.

$$
A X=B
$$

onde a matriz $\boldsymbol{A}$ contém os termos de $\boldsymbol{H}$ e $\boldsymbol{G}$, correspondentes às grandezas incógnitas no contorno, e o termo $\boldsymbol{B}$ contém os termos de $\boldsymbol{H}$ e $\boldsymbol{G}$ de variáveis prescritas no contorno, tomando-se o cuidado para a mudança de sinais, onde há as trocas dos coeficientes de influênica das matrizes $\boldsymbol{H}$ e $\boldsymbol{G}$, respectivamente. Com a utilização de técnicas de solução de sistemas algébricos lineares, encontram-se as variáveis incógnitas $\boldsymbol{X}$.

Para se colocar os deslocamentos internos da estrutura, basta escrever a equação (3.54) para pontos internos, considerando para isto $c_{i j}=1$, e, utilizando-se dos valores incógnitos e prescritos da equação (3.57), obtém-se:

$$
\boldsymbol{u}=-\boldsymbol{H U}+\boldsymbol{G P}
$$

Para se conhecer as tensões em pontos do domínio, faz-se o somatório das integrais em cada elemento da equação (3.51), obtendo-se.

$$
\sigma_{i j}=\sum_{E=1}^{N E} \int_{\Gamma_{E}}\left[D_{i j k}^{*}(x, \xi) \phi_{m}^{E} p_{k}(x)-S_{i j k}^{*}(x, \xi) \phi_{m}^{E} u_{k}(x)\right] d \Gamma_{E}
$$


que pode ser escrita em forma matricial:

$$
\sigma=-S U+D P
$$

Como pode ser visto nas equações (3.58) e (3.60), as equações do domínio estão escritas em função das variáveis de contorno, não sendo necessário a resolução de sistemas lineares.

\subsection{Integrais Exata}

Neste trabalho foi adotada a integração analítica de todos os elementos de contorno. Na integração numérica utilizam-se pontos de Gauss intermediários, onde cada ponto tem uma função de peso equivalente e, quanto mais dividido o elemento, mais precisa se torna a integração. Por se tratar de um processo de aproximação, as integrais carregam erros e o tempo de processamento é aumentado em função da quantidade de pontos utilizados na integração.

Ao contrário do que ocorre nas integrais numéricas, na integração exata as expressões são integradas analiticamente, evitando-se assim erros de aproximação e diminuindo-se consideravelmente o tempo de processamento computacional.

Nesse trabalho não foram explicitadas as integrais analíticas singulares, ou seja, aquelas que o ponto fonte está no elemento integrado, pois essas expressões já foram largamente descritas em vários trabalhos (Wutzow, 2003). Nas integrais onde o ponto fonte não coincide com o elemento, devem-se considerar três posições do ponto fonte em relação ao elemento a ser integrado, como mostra a figura (3.8). Nas duas primeiras posições, o ponto fonte está alinhado com o elemento e, portanto, só há duas situações para este caso, ou o ponto fonte está à frente do elemento ou ele está atrás. No terceiro caso, o ponto fonte não está alinhado com o elemento.

A figura (3.9) mostra os parâmetros e os limites de integração das integrais analíticas, onde $\theta_{2}$ e $\theta_{1}$ são os limites de integração relativos aos nós 2 e 1 , respectivamente. A reta $a$ é a distância entre o ponto fonte $\xi$ e a reta que contém o elemento. 

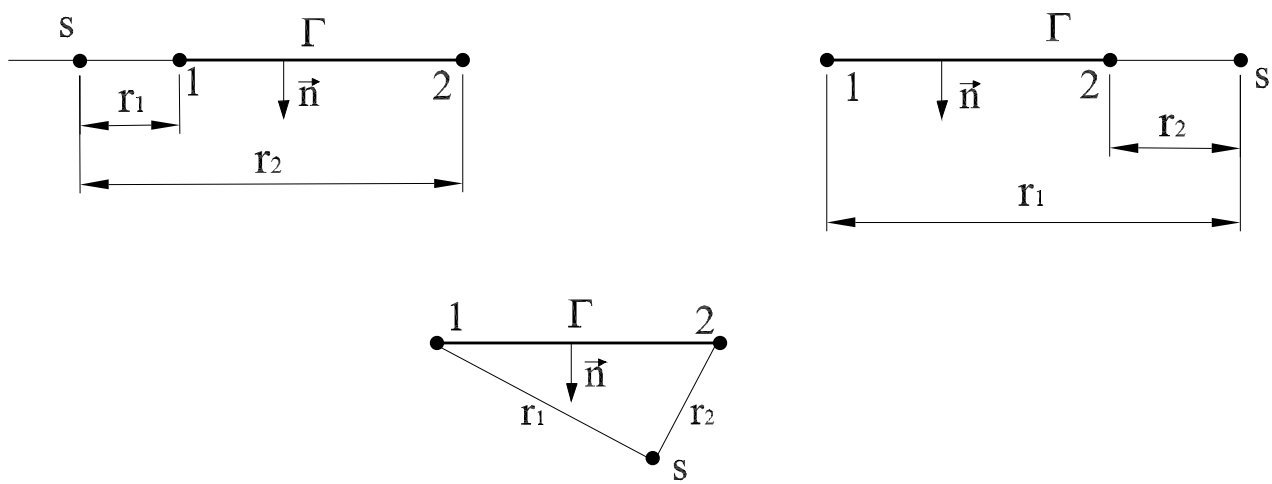

Fig. 3.8: Possíveis posições do ponto fonte em integração analítica não singular

O eixo $x-y$ é a coordenada local do elemento onde, neste trabalho, foi adotada a orientação do eixo $x$ coincidente com a normal do elemento, e o eixo $y$ coincidente com a direção do elemento.

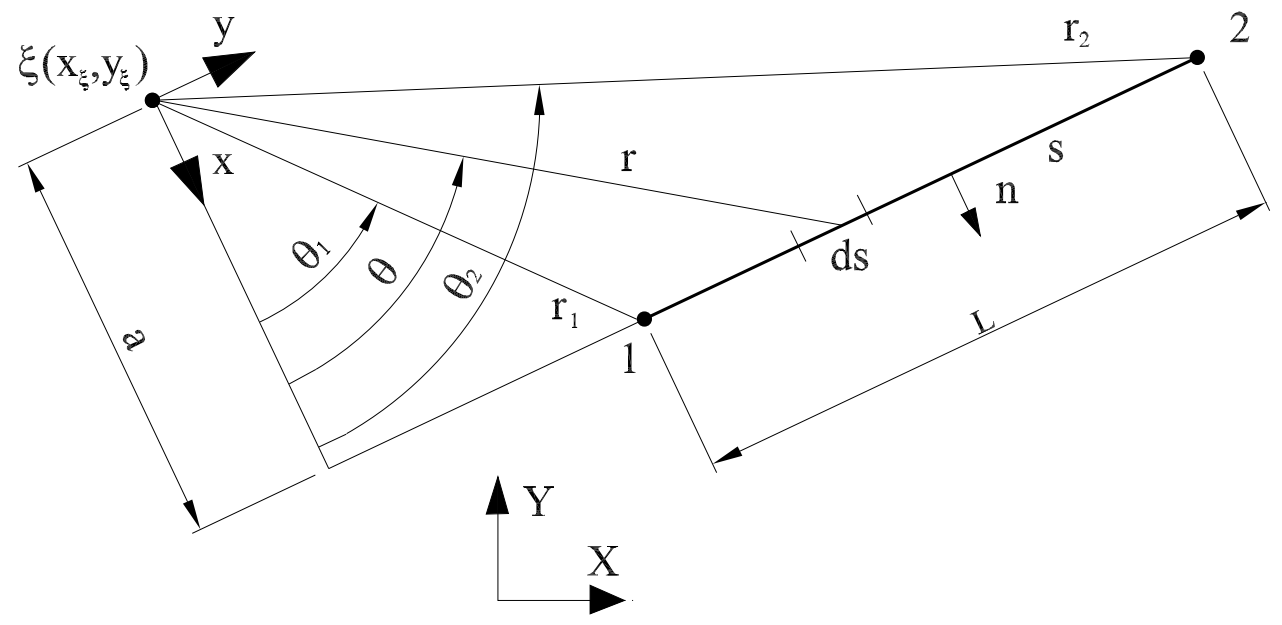

Fig. 3.9: Coordenadas locais para integração analítica com ponto fonte não alinhado com o elemento

A integração, neste caso, é feita seguindo a orientação do eixo de coordenadas locais do elemento. Pela figura (3.9), conclui-se que:

$$
\cos (\theta)=\frac{a}{r} \quad \text { ou seja } \quad r=\frac{a}{\cos (\theta)}
$$




$$
\begin{array}{r}
\operatorname{sen}(\theta)=\frac{y}{r} \\
\operatorname{tg}(\theta)=\frac{y-y_{\xi}}{a} \quad \text { ou seja } \quad y=a * \operatorname{tg}(\theta)+y_{\xi}
\end{array}
$$

sendo, o termo $y_{\xi}$, a posição no eixo $y$ em coordenadas locais do ponto fonte $\xi$, e o termo $y$, as ordenadas dos pontos do elemento em relação ao eixo local $y$.

A parte infinitesimal, do elemento a ser integrado, pode ser escrita em função do ângulo local $\theta$ e do valor fixo $a$, com isto, tem-se a parte infinitesimal $d s$ do elemento escrita em função do eixo local $y$ e do ângulo $\theta$ :

$$
d s=d y=a * \sec ^{2}(\theta) d \theta=\left(\frac{a}{\cos ^{2}(\theta)}\right) d \theta
$$

onde os termos das soluções fundamentais de deslocamentos, forças de superfície e tensões podem ser escritos em função das coordenadas locais:

$$
\begin{array}{rr}
r, 1=\cos (\theta) & r,_{2}=\operatorname{sen}(\theta) \\
n_{1}=\cos (n, x)=\cos \left(0^{\circ}\right)=1 & n_{2}=\cos (n, y)=\cos \left(90^{\circ}\right)=0 \\
r_{,_{n}}=\frac{\partial r}{\partial n}=\frac{\partial r}{\partial x} n_{x}+\frac{\partial r}{\partial y} n_{y}=r,{ }_{1} n_{1}+r,,_{2} n_{2}=\cos (\theta)
\end{array}
$$

Da mesma forma, as funções aproximadoras serão transformadas em coordenadas locais e serão integradas em $\theta$. Neste caso, ao contrário da integração numérica, nas funções aproximadoras não será usado um sistema de coordenadas paramétricas para os elementos, e a origem do sistema local de coordenadas é o nó 1 do elemento.

As funções aproximadoras podem ser escritas como:

$$
\phi_{1}=1-\frac{y_{p}}{L}=1-\left(\frac{y_{\xi}}{L}-a \frac{\operatorname{tg}(\theta)}{L}\right)
$$




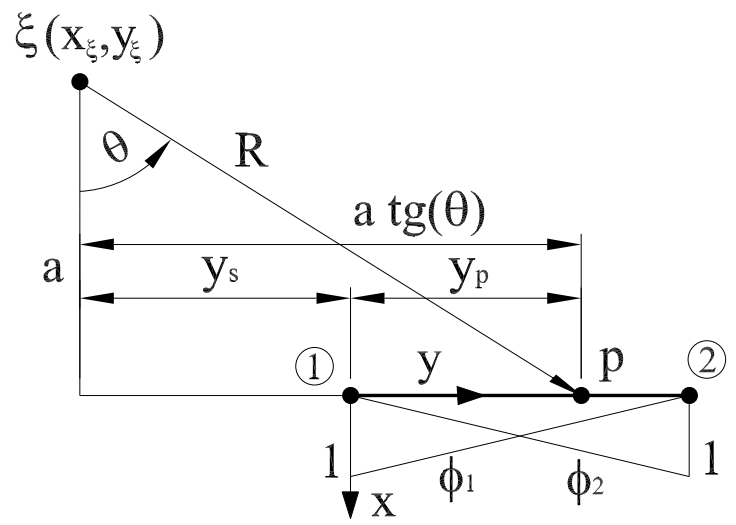

Fig. 3.10: Funções aproximadoras e variáveis locais para integração

$$
\phi_{2}=\frac{y_{p}}{L}=a \frac{\operatorname{tg}(\theta)}{L}-\frac{y_{\xi}}{L}
$$

onde $y_{p}$ é a coordenada local do ponto fonte $p$ e $L$ é o comprimento do elemento. Os valores de $y_{p}, y_{\xi}$ e $a \cdot \operatorname{tg}(\theta)$ devem ser tomados sempre com valores positivos para as equações das funções aproximadoras.

Com todos os termos das integrais das soluções fundamentais 'H', 'G','D' e 'S' escritas em função das coordenadas locais, segundo as expressões, basta integrar estes termos na variável $\theta$ entre os limites de integração entre $\theta_{2}$ do segundo nó do elemento e $\theta_{1}$ do nó inicial do elemento.

No caso do ponto fonte estar alinhado com o elemento, a integral foi feita pelo raio $d \Gamma=d r$, sendo todos os parâmetros escritos em função do eixo local da figura (3.11).

As funções aproximadoras, para o caso do ponto fonte estar alinhado atrás do elemento, podem ser escritas adimensionalmente com as coordenadas locais do elemento sob a forma:

$$
\begin{array}{r}
\phi_{1}=1-\frac{r_{p}}{L}=1-\frac{r}{L}+\frac{r_{\xi}}{L} \\
\phi_{2}=\frac{r_{p}}{L}=\frac{r}{L}-\frac{r_{\xi}}{L}
\end{array}
$$




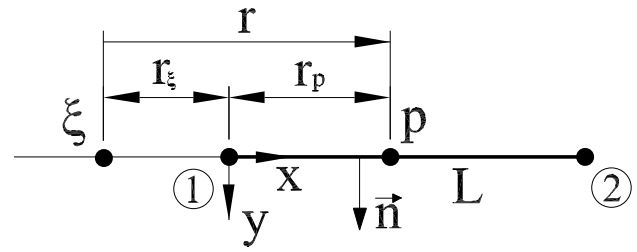

(a)

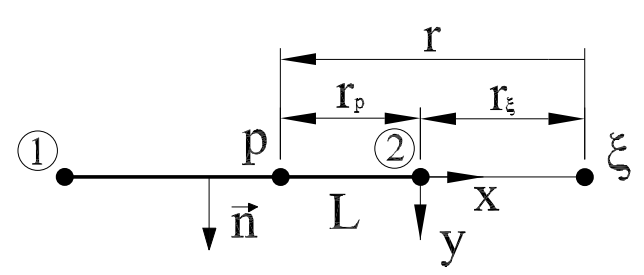

(b)

Fig. 3.11: Funções aproximadoras em elementos alinhados com o ponto fonte a) Ponto fonte atrás do elemento b) Ponto fonte à frente do elemento

Como no caso das equações (3.68) e (3.69), os valores de $r_{p}, r_{\xi}$ e $r$, são escritos sempre com valores positivos.

Por ser as equações fundamentais integradas em coordenadas locais, o ponto fonte e os nós do elemento dever ser escritos nas coordenadas locais do elemento. Após a integração analítica, o resultado deve ser escrito no sistema global para a montagem do sistema algébrico de equações de contorno.

As matrizes $\boldsymbol{H}_{\boldsymbol{i j}}$ e $\boldsymbol{G}_{\boldsymbol{i} \boldsymbol{j}}$, se relacionam com deslocamentos e forças de superfície na direção de $i$, respectivamente, causados por uma força unitária na direção $j$, por isso a matriz de rotação se relaciona com a posição das coordenadas globais com locais por uma matriz quadrada, como mostra a figura (3.12).

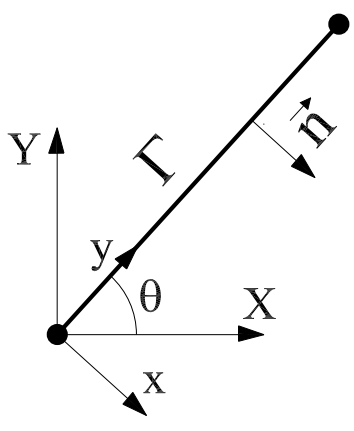

Fig. 3.12: Relação entre eixos globais e eixo local do elemento

onde a matriz de rotação pode ser escrita por: 


$$
[C]=\left[\begin{array}{cc}
\operatorname{sen}(\theta) & -\cos (\theta) \\
\cos (\theta) & \operatorname{sen}(\theta)
\end{array}\right]
$$

sendo a equação de $H$ e $G$ rotacionadas para a posição global:

$$
\begin{aligned}
{\left[H^{g}\right] } & =[C]^{T}\left[H^{l}\right][C] \\
{\left[G^{g}\right] } & =[C]^{T}\left[G^{l}\right][C]
\end{aligned}
$$

onde os índices $g$ e $l$ se referem as matrizes $\boldsymbol{H}$ e $\boldsymbol{G}$ nas coordenadas globais e locais, respectivamente, e o termo $[C]^{T}$ é a matriz transposta de $[C]$.

No caso das integrais $\boldsymbol{D}_{\boldsymbol{i j \boldsymbol { k }}}$ e $\boldsymbol{S}_{\boldsymbol{i j \boldsymbol { k }}}$, que relacionam deformação e tensão, respectivamente, segundo o índice $i j$ causados por uma força unitária aplicada na direção de $k$, a matriz de rotação de eixos deve ser escrita com a relação mostrada em (2.15), para multiplicar as tensões nas direções de $i j$, por uma matriz da equação (3.72) que multiplica os termos da carga unitária nas direções de $k$. Com isso e segundo a relação entre eixos da figura (3.12), tem-se a equação:

$$
\begin{aligned}
{\left[D^{g}\right] } & =[B]\left[D^{l}\right][C] \\
{\left[S^{g}\right] } & =[B]\left[S^{l}\right][C]
\end{aligned}
$$

a matriz de rotação $[B]$ é escrita como:

$$
[B]=\left[\begin{array}{ccc}
\operatorname{sen}(\theta)^{2} & 2 \operatorname{sen}(\theta) \cos (\theta) & \cos (\theta)^{2} \\
-\operatorname{sen}(\theta) \cos (\theta) & \operatorname{sen}(\theta)^{2} \cos (\theta)^{2} & \operatorname{sen}(\theta) \cos (\theta) \\
\cos (\theta)^{2} & -2 \cos (\theta) \operatorname{sen}(\theta) & \operatorname{sen}(\theta)^{2}
\end{array}\right]
$$




\subsection{Técnicas de Subelementação}

Uma das técnicas mais utilizadas, principalmente em integração numérica, é a técnica da integração por subelementos. Essa técnica deve ser utilizada quando o ponto fonte se encontra muito próximo do elemento a ser integrado, pois nessa posição haverá problemas de quase singularidade, ou seja, os núcleos das integrais passam a ter gradientes muito elevados e fazem a integral numérica divergir.

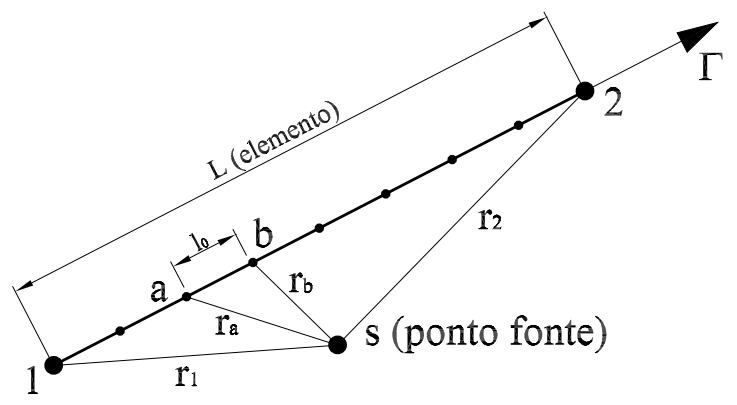

Fig. 3.13: Subelementação padronizada

Tal técnica consiste basicamente em dividir o elemento de integração em subelementos menores (de comprimento padronizado ou progressivo). Nos de tamanho padronizados, figura(3.13), os elementos são divididos em subelementos de tamanhos iguais e é feita a integração sobre cada subelemento e, em seguida, seus valores são interpolados para os valores dos nós da extremidade do elemento.

$\mathrm{Na}$ subelementação progressiva, os elementos são divididos em subelementos cujos tamanhos diminuem a medida em que os subelementos estejam mais próximos do ponto fonte. Isso faz com que diminuam os gradientes das funções em limites muito próximos ao ponto fonte.

A figura (3.15) mostra as coordenadas adimensionais de cada subelemento em relação as coordenadas adimensionais do elemento.

Seja a relação:

$$
\int_{\Gamma_{j}} f\left(\Gamma_{j}\right) \phi_{n} d \Gamma_{j}=\sum_{j=1}^{N S E} \int f\left(\Gamma_{j i}\right) \phi_{n} d \Gamma_{j i}
$$




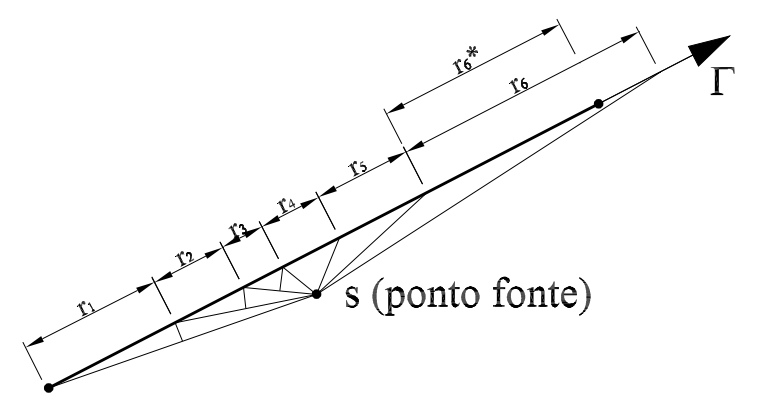

Fig. 3.14: Subelementação progressiva

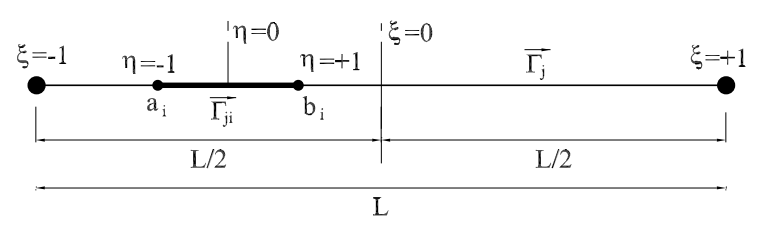

Fig. 3.15: Coordenadas adimensionais $\eta$ e $\xi$.

onde NSE é o número de subelementos por elemento. Transformando as coordenadas do elemento em adimensionais, tem-se:

$$
\begin{gathered}
\Gamma=a_{i} \rightarrow \eta=-1 \\
\Gamma=b_{i} \rightarrow \eta=1
\end{gathered}
$$

Logo:

$$
\int_{\Gamma_{j}} f\left(\Gamma_{j}\right) \phi_{\eta} d \Gamma_{j}=\sum_{i=1}^{N S E} \frac{L_{i}}{2} \int_{-1}^{1} f(\eta) \phi_{\eta} d \eta
$$

Pelo fato do intervalo de integração estar em função da coordenada adimensional $\eta[-1 ;+1]$ e, sendo a função de forma escrita em coordenada adimensional $\xi$, há a necessidade de se fazer a correlação entre as duas coordenadas adimensionais. 


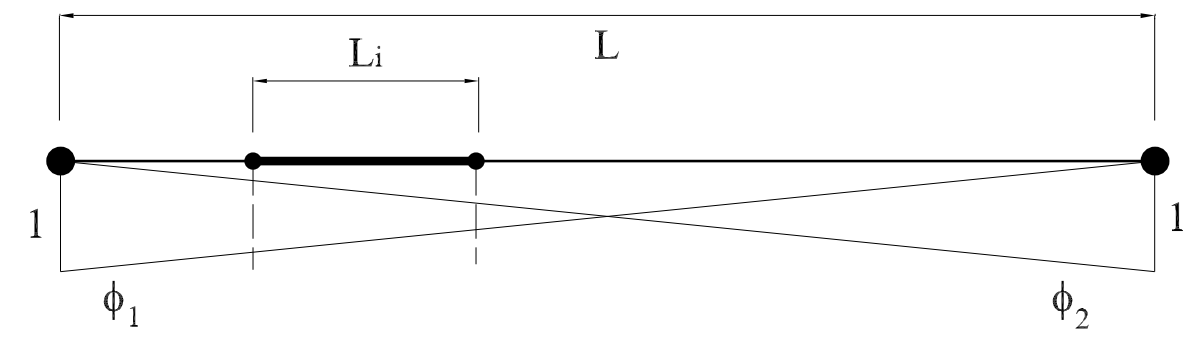

Fig. 3.16: Funções de forma do elemento.

Para se saber se é necessária a utilização ou não de subelementos, procede-se da forma mostrada na figura (3.17), onde se tem um elemento que será integrado numericamente, sendo o ponto $S$ próximo ao elemento.

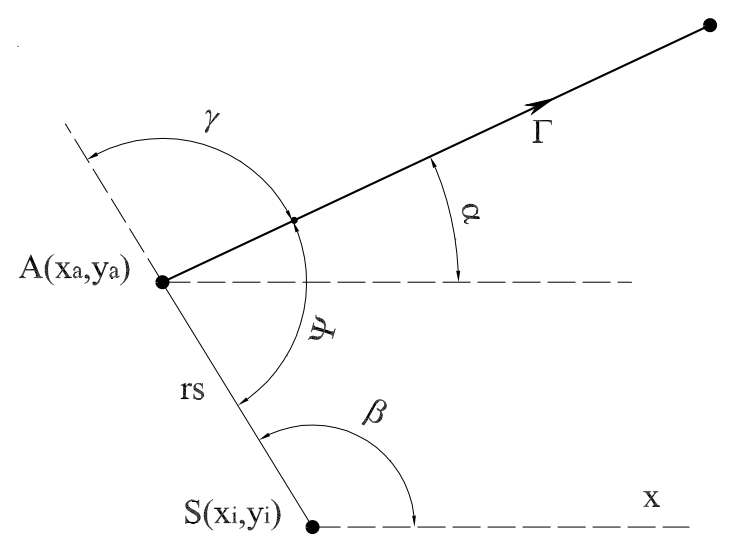

Fig. 3.17: Determinação dos ângulos para o sub-elemento.

Em um primeiro passo, calcula-se a distância $(r s)$ do ponto fonte ao início do elemento, dada por:

$$
r s=\sqrt{\left(x_{i}-x_{a}\right)^{2}+\left(y_{i}-y_{a}\right)^{2}}
$$

Calculam-se também o seno e o cosseno do ângulo formado por esta reta $(r s)$, com o eixo $x$ : 


$$
\begin{aligned}
& \cos (\beta)=\frac{x_{a}-x_{i}}{r s} \\
& \operatorname{sen}(\beta)=\frac{y_{a}-y_{i}}{r s}
\end{aligned}
$$

Pela figura (3.17), pode-se concluir que:

$$
\gamma=\beta-\alpha \Rightarrow \cos (\gamma)=\cos (\beta-\alpha)=\cos (\alpha) \cos (\beta)+\operatorname{sen}(\alpha) \operatorname{sen}(\beta)
$$

onde o ângulo $\Psi$ da figura (3.17) pode ser encontrado por:

$$
\Psi=\pi-\gamma
$$

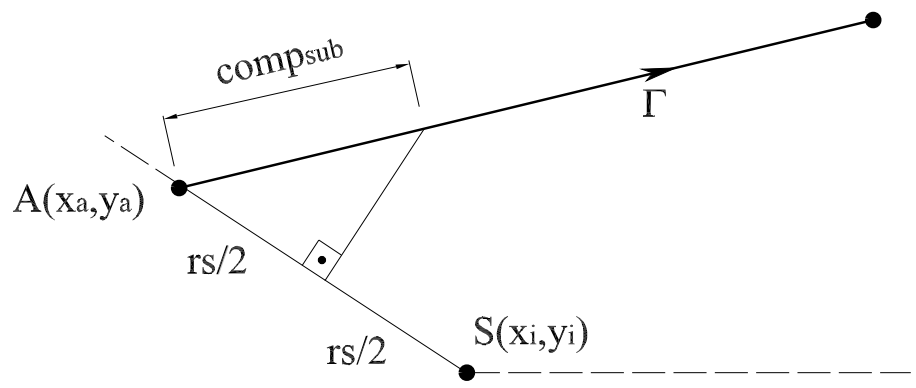

Fig. 3.18: Determinação do comprimento para o sub-elemento.

Se o ângulo $\Psi$ for menor que $60^{\circ}$, então o comprimento do sub-elemento será dado pela interseç̧ão da mediatriz da distância $r s$ com o elemento de integração, segundo a figura (3.18).

sendo o comprimento do sub-elemento $\left(c o m p_{s u b}\right)$, dado pela relação: 


$$
\cos (\Psi)=\frac{\frac{r s}{2}}{c o m p_{s u b}} \Rightarrow c o m p_{s u b}=\left|\frac{r s}{2 \cos (\gamma)}\right|
$$

Como $\Psi=\pi-\gamma, \log$ o $\cos (\Psi)=-\cos (\gamma)$. Quando o ângulo $\Psi$ for maior que $60^{\circ}$, então o comprimento do sub-elemento $\left(c\right.$ omp $\left.p_{\text {sub }}\right)$ será igual ao comprimento da distância $r s$, como mostra a figura (3.19)

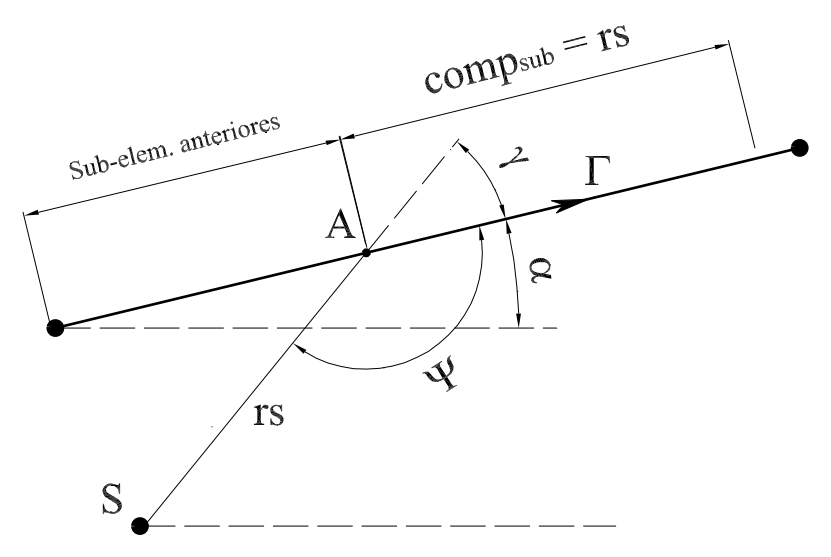

Fig. 3.19: Comprimento do sub-elemento para $\Psi$ maior que $60^{\circ}$

Repete-se este procedimento até o somatório dos subelementos ser maior ou igual ao elemento de integração. Caso o sub-elemento seja maior que o que resta para completar o elemento de integração, então este último sub-elemento terá comprimento igual ao que resta para completar o somatório, o que acontece com $r_{6}$ na figura (3.14).

Vale mencionar aqui que, a posição onde se pode utilizar a subelementação deve ser apenas quando o nó estiver muito próximo ao elemento e que, existem parâmetros que determinam a distância ideal para se utilizar tal técnica.

Para se ter maiores referências sobre a técnica de sub-elemetação, na EESC deve-se consultar (Chaves, 1997), onde pode ser visto com mais detalhes o desenvolvimento da técnica bem como de algumas análises de precisão. 


\section{ANÁLISE DE REGIÕES COM MULTIDOMÍNIOS VIA MEC}

\subsection{Introdução}

Neste capítulo, será vista a análise de sólidos que possuem mais de uma região com características mecânicas diferentes, utilizando-se o Método dos Elementos de Contorno. Será vista também a técnica de condensação de domínios, onde um domínio muito esbelto é transformado em uma sub-região linear, originando assim um sistema algébrico mais estável.

Uma primeira formulação, chamada de formulação singular, consiste em se eliminar as incógnitas de forças de superfície da interface entre os domínios diferentes, através das condições de equilíbrio desta região, onde somente haverá incógnitas de deslocamentos.

Na outra formulação, que é chamada de formulação hipersingular, as incógnitas de deslocamentos são retiradas do sistema algébrico, através da compatibilidade de deslocamentos entre os nós da interface, devendo apenas apresentar como incógnitas as forças de superfície da interface.

Para domínios muito esbeltos será adotada, neste capítulo, a técnica de condensação de domínios. Numa primeira hipótese, considera-se que o deslocamento é constante entre as faces da inclusão, sendo assim formulado um comportamento de fibra para este sub-domínio. Em outra análise, considera-se que os deslocamentos variem linearmente através da espessura da inclusão, tendo esta agora um comportamento de viga. Para esta última hipótese, devem-se escrever equações de rotação para os nós centrais das inclusões.

Por fim serão mostrados exemplos numéricos, comparando tais técnicas com técnicas convencionais de análise de regiões com multidomínios e também com a 
técnica de acoplamento MEC/MEF. Na análise de sub-regiões muito esbeltas, serão analisadas sub-regiões com rigidez maior que a do domínio principal e também de sub-regiões com rigidez quase nula, simulando o comportamento de uma fratura. Tais resultados serão comparados com soluções teóricas e com os resultados obtidos por outros métodos numéricos.

\subsection{Sub-regiões}

\subsubsection{Formulação Singular}

Considera-se a figura (4.1), onde o sólido é formado por duas sub-regiões, $\Omega_{1}$ e $\Omega_{2}$, com propriedades elásticas distintas.

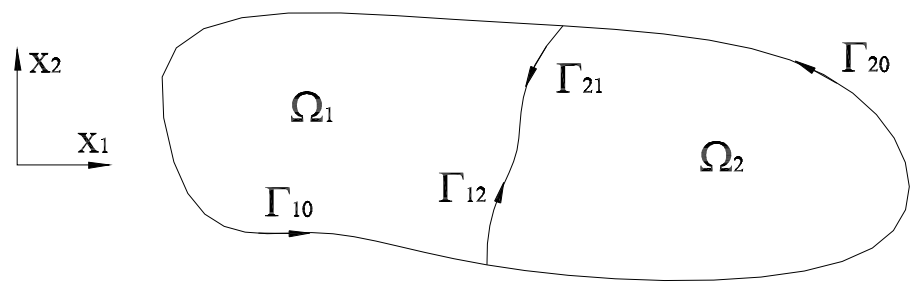

Fig. 4.1: Região com multidomínios

Os desenvolvimentos a serem apresentados são gerais, tendo sido tomado o caso particular de domínio bidimensional e com número limitado de sub-regiões apenas por simplicidade. Para a configuração adotada, cada uma das sub-regiões pode ser considerada isoladamente, levando às seguintes relações:

$$
\begin{aligned}
& \int_{\Omega_{1}} \sigma_{i j}^{1} \epsilon_{i j}^{1 *} d V=\int_{\Omega_{1}} \sigma_{i j}^{1 *} \epsilon_{i j}^{1} d V \\
& \int_{\Omega_{2}} \sigma_{i j}^{2} \epsilon_{i j}^{2 *} d V=\int_{\Omega_{2}} \sigma_{i j}^{2 *} \epsilon_{i j}^{2} d V
\end{aligned}
$$

onde os símbolos ' 1 *' e '2*', são usados para identificar soluções fundamentais definidas com as características das sub-regiões $\Omega_{1}$ e $\Omega_{2}$, respectivamente; $\sigma_{i j}$ e $\epsilon_{i j}$ representam os campos de tensões e deformações observados nessas sub-regiões. 
Fazendo-se as integrações por partes, em (4.1), obtêm-se as representações integrais em deslocamentos para pontos de colocação quaisquer, isto é, no interior de cada uma, em pontos não pertencentes ao domínio, no contorno ou sobre a interface.

$$
\begin{aligned}
& c_{i j} u_{j}^{1}=-\int_{\Gamma_{10}} H_{i j}^{1 *} u_{j}^{1} d \Gamma-\int_{\Gamma_{12}} H_{i j}^{1 *} u_{j}^{1} d \Gamma+\int_{\Gamma_{10}} G_{i j}^{1 *} p_{j}^{1} d \Gamma+\int_{\Gamma_{12}} G_{i j}^{1 *} p_{j}^{1} d \Gamma \\
& c_{i j} u_{j}^{2}=-\int_{\Gamma_{20}} H_{i j}^{2 *} u_{j}^{2} d \Gamma-\int_{\Gamma_{21}} H_{i j}^{2 *} u_{j}^{2} d \Gamma+\int_{\Gamma_{20}} G_{i j}^{2 *} p_{j}^{2} d \Gamma+\int_{\Gamma_{21}} G_{i j}^{2 *} p_{j}^{2} d \Gamma
\end{aligned}
$$

onde os termos $\Gamma_{10}$ e $\Gamma_{20}$ dizem respeito ao contorno apenas da sub-região 1 e 2 , respectivamente, e $\Gamma_{12}$ e $\Gamma_{21}$ se referem ao contorno da interface, sendo que a ordem do sub-índice indica o caminho da integração, como mostra a figura (4.1).

Multiplicando-se a expressão (4.2) por $\gamma_{1}$ e (4.3) por $\gamma_{2}$, sendo $\gamma_{i}$ o módulo de elasticidade transversal do material, e atentando-se para o equilíbrio de forças na interface $p_{j}^{1}=-p_{j}^{2}$, a compatibilidade de deslocamentos na mesma $u_{j}^{1}=u_{j}^{2}$, e substituindo-se o caminho de integração da equação (4.3) na região da interface pela mesma direção de integração da equação (4.2), ou seja $\Gamma_{12}$, tem-se:

$$
\begin{array}{r}
\gamma_{1} c_{i j} u_{j}^{1}=-\gamma_{1} \int_{\Gamma_{10}} H_{i j}^{1 *} u_{j}^{1} d \Gamma-\gamma_{1} \int_{\Gamma_{12}} H_{i j}^{1 *} u_{j}^{1} d \Gamma+ \\
+\int_{\Gamma_{10}} \bar{G}_{i j}^{1 *} p_{j}^{1} d \Gamma+\int_{\Gamma_{12}} \bar{G}_{i j}^{1 *} p_{j}^{1} d \Gamma \\
\gamma_{2} c_{i j} u_{j}^{1}=-\gamma_{2} \int_{\Gamma_{20}} H_{i j}^{2 *} u_{j}^{2} d \Gamma-\gamma_{2} \int_{\Gamma_{12}} H_{i j}^{2 *} u_{j}^{1} d \Gamma+ \\
+\int_{\Gamma_{20}} \bar{G}_{i j}^{2 *} p_{j}^{2} d \Gamma-\int_{\Gamma_{12}} \bar{G}_{i j}^{2 *} p_{j}^{1} d \Gamma
\end{array}
$$

onde os termos $\bar{G}_{i j}^{k *}$, com $k=1,2$, são as soluções fundamentais sem os módulos de elasticidade transversais $\gamma_{k}$, pois foram eliminados pela multiplicação dos mesmos módulos nas equações (4.4) e (4.5). 
Para os pontos fonte em qualquer posição, somando-se (4.4) e (4.5), obtém-se de uma forma geral:

$$
\frac{\gamma_{k}+\gamma_{l}}{\gamma_{k}} c_{i j} u_{j}^{k}=\sum_{k=1}^{N_{s}} \sum_{\substack{l=0 \\ k \neq l}}^{N_{s}} \frac{-\gamma_{k}+\gamma_{l}}{\gamma_{k}} \int_{\Gamma_{k l}} H_{i j}^{k *} u_{j}^{k} d \Gamma+\sum_{k=1}^{N_{s}} \int_{\Gamma_{k 0}} G_{i j}^{k *} p_{j}^{k} d \Gamma
$$

onde $k$ define as sub-regiões e $N_{s}$ é o número de sub-regiões. O índice $l$ diz respeito a sub-região de interface. Quando esse termo é zero, o ponto fonte pode estar em um contorno externo de uma sub-região ou internamente a essa mesma sub-região. Para valores de $l$ diferentes de zero, significa dizer que se está na interface com o índice $k$, onde os dois sub-índices indicam a posição desta interface.

O termo independente, $c_{i j}$, é dado pela mesma expressão já deduzida para o caso de problemas elásticos. No caso de pontos sobre a interface, o termo independente é obtido seguindo-se os mesmos passos utilizados para a dedução da Eq. (4.6). A dedução pode ser estendida para o caso geral elástico.

A partir da equação (4.6) podem-se obter as representações integrais de deformação e de tensão, derivando-se e utilizando-se a lei de Hooke. Assim, a representação integral das tensões em pontos internos fica dada por:

$$
\sigma_{i j}=\sum_{k=1}^{N_{s}} \sum_{\substack{l=0 \\ k \neq l}}^{N_{s}} \frac{\gamma_{k}-\gamma_{l}}{\gamma_{k}} \int_{\Gamma_{k}} S_{i j m}^{k *} u_{m} d \Gamma+\sum_{k=1}^{N_{s}} \int_{\Gamma} D_{i j m}^{k} p_{m}^{k} d_{\Gamma}
$$

Nesta formulação, o número de incógnitas na interface se reduz somente às incógnitas de deslocamento, diminuindo em duas vezes a quantidade de incógnitas da interface, em relação à análise tradicional de sub-região.

As integrais das equações (4.6) e (4.7), serão aproximados no contorno e nas interfaces das sub-regiões por elementos de contorno onde, os deslocamentos e forças de superfície, serão aproximados por funções aproximadoras lineares. Foi utilizada integração analítica para todos os elementos, que serão efetuadas em coordenadas 
locais do elemento a ser integrado, como mostra a figura (3.12), e posteriormente o resultado será rotacionado para o sistema global de coordenadas cartesianas.

Reunindo-se todas as contribuições das equações (4.6) de todos os elementos, pode-se escrever a equação matricial do método dos elementos de contorno:

$$
\left[\begin{array}{ll}
H_{c c} & H_{c i} \\
H_{i c} & H_{i i}
\end{array}\right]\left\{\begin{array}{l}
U_{c} \\
\boldsymbol{u}_{i}
\end{array}\right\}=\left[\begin{array}{l}
G_{c c} \\
G_{i c}
\end{array}\right]\left\{\boldsymbol{P}_{c}\right\}
$$

onde os índices $c$ e $i$ referem-se a pontos do contorno e interface, respectivamente.

\subsubsection{Formulação Hiper-singular}

Como pode ser visto na figura (4.1), pode-se escrever para o domínio $\Omega_{1}$ e $\Omega_{2}$, uma formulação que pode ser estendida a qualquer quantidade de subdomínios contidos na estrutura. Utilizando-se as equações de forças de superfície para os nós, tanto da interface quanto do contorno, têm-se para as duas sub-regiões.

$$
\begin{aligned}
& \frac{1}{2} p_{j}^{1}=-\int_{10} S_{i j l}^{1 *} n_{j} u_{l}^{1} d \Gamma-\int_{\Gamma_{12}} S_{i j l}^{1 *} n_{j} u_{l}^{1} d \Gamma+\int_{\Gamma_{10}} D_{i j l}^{1 *} n_{j} p_{l}^{1} d \Gamma+\int_{\Gamma_{12}} D_{i j l}^{1 *} n_{j} p_{l}^{1} d \Gamma \\
& \frac{1}{2} p_{j}^{2}=-\int_{20} S_{i j l}^{2 *} n_{j} u_{l}^{2} d \Gamma-\int_{\Gamma_{21}} S_{i j l}^{2 *} n_{j} u_{l}^{2} d \Gamma+\int_{\Gamma_{20}} D_{i j l}^{2 *} n_{j} p_{l}^{2} d \Gamma+\int_{\Gamma_{21}} D_{i j l}^{2 *} n_{j} p_{l}^{2} d \Gamma
\end{aligned}
$$

onde, $S_{i j l}^{*}$ e $D_{i j l}^{*}$ são as soluções fundamentais para tensão e, $n_{j}$ é a normal da interface entre domínios e também do contorno.

Multiplicando-se a expressão (4.9) por $1 / \gamma_{1}$ e (4.10) por $1 / \gamma_{2}$, levando-se em conta o equilíbrio de forças na interface $p_{j}^{1}=-p_{j}^{2}$, a compatibilidade de deslocamentos na mesma, $u_{j}^{1}=u_{j}^{2}$ e substituindo-se o caminho de integração da equação (4.10) na região da interface, pela mesma direção de integração da equação (4.9), ou seja $\Gamma_{12}$, tem-se: 


$$
\begin{aligned}
& \frac{1}{2} p_{j}^{1} \frac{1}{\gamma_{1}}=-\int_{10} \bar{S}_{i j l}^{1 *} n_{j} u_{l}^{1} d \Gamma-\int_{\Gamma_{12}} \bar{S}_{i j l}^{1 *} n_{j} u_{l}^{1} d \Gamma+ \\
&+ \frac{1}{\gamma_{1}} \int_{\Gamma_{10}} D_{i j l}^{1 *} n_{j} p_{l}^{1} d \Gamma+\frac{1}{\gamma_{1}} \int_{\Gamma_{12}} D_{i j l}^{1 *} n_{j} p_{l}^{1} d \Gamma \\
&-\frac{1}{2} p_{j}^{1} \frac{1}{\gamma_{2}}=-\int_{20} \bar{S}_{i j l}^{2 *} n_{j} u_{l}^{2} d \Gamma+\int_{\Gamma_{12}} \bar{S}_{i j l}^{2 *} n_{j} u_{l}^{2} d \Gamma+ \\
&+\frac{1}{\gamma_{2}} \int_{\Gamma_{20}} D_{i j l}^{2 *} n_{j} p_{l}^{2} d \Gamma+\frac{1}{\gamma_{2}} \int_{\Gamma_{12}} D_{i j l}^{2 *} n_{j} p_{l}^{1} d \Gamma
\end{aligned}
$$

onde os termos $\bar{S}_{i j l}^{k *}$, com $k=1,2$, são as soluções fundamentais sem os módulos de elasticidade transversal $\gamma_{k}$, pois foram eliminados pela multiplicação de $1 / \gamma_{k}$ nas equações (4.11) e (4.12).

Para os pontos fonte em qualquer posição, somando-se (4.11) e (4.12), obtémse:

$$
\frac{\gamma_{l}-\gamma_{m}}{2 \gamma_{m}} p_{j}^{m}=\sum_{m=1}^{N_{s}} \sum_{\substack{l=0 \\ m \neq l}}^{N_{s}} \frac{\gamma_{m}+\gamma_{l}}{\gamma_{m}} \int_{\Gamma_{m l}} D_{i j k}^{m *} n_{j} p_{j}^{m} d \Gamma+\sum_{m=1}^{N_{s}} \int_{\Gamma_{m 0}} S_{i j k}^{m *} n_{j} u_{j}^{m} d \Gamma
$$

No caso do ponto fonte estar localizado em um contorno de uma só sub-região, basta considerar o termo $\gamma_{l}$ igual a zero.

As integrais da equação (4.13) serão aproximadas no contorno e nas interfaces das sub-regiões por elementos de contorno com funções aproximadoras lineares. Foi utilizada integração analítica para todos os elementos, que serão efetuadas em coordenadas locais do elemento a ser integrado, como mostra a figura (3.12), e posteriormente o resultado será rotacionado para o sistema global de coordenadas cartesianas.

Reunindo-se todas as contribuições das equações (4.13), de todos os elementos, pode-se escrever a equação matricial do método dos elementos de contorno: 


$$
\left[\begin{array}{cc}
S_{c c} n & D_{c i} n \\
S_{i c} n & D_{i i} n
\end{array}\right]\left\{\begin{array}{l}
U_{c} \\
p_{i}
\end{array}\right\}=\left[\begin{array}{c}
D_{c c} n \\
D_{i c} n
\end{array}\right]\left\{P_{c}\right\}
$$

onde o vetor $\boldsymbol{n}$ é a normal de cada elemento integrado.

Podem-se ter quatro combinações das formulações singulares (4.6) e hipersingulares (4.13) em uma mesma análise. Pode ser utilizada a formulação singular no contorno e na interface, como mostra a expressão (4.8) e a hiper-singular também no contorno e na interface. Pode-se ter também uma combinação das duas formulações, como a formulação singular no contorno e a hiper-singular na interface e a formulação hiper-singular no contorno e a singular na interface.

\subsection{Técnicas de Condensação de Domínios}

Em sub-regiões muito delgadas, como as posições dos pontos das faces estão muito próximas e, esta distância é muito pequena se comparada com a dimensão do domínio principal, pode-se acarretar dependência linear no sistema algébrico de equações, comprometendo a precisão das respostas.

Para contornar este problema, adota-se a técnica de condensação de domínios, que consiste em se transformar um domínio 2D em um domínio linear. No centro deste domínio são introduzidos novos nós (pontos de colocação). Assim pode-se admitir uma aproximação dos deslocamentos e tensões na direção normal a subregião delgada.

\subsubsection{Transformação de Domínios 2D em Fibras}

No caso da condensação do subdomínio em fibra, os deslocamentos serão admitidos constantes ao longo da espessura $h$ da fibra, como mostra a figura (4.2). onde o nó $S$ é o nó central do subdomínio, e os nós $S_{d}$ e $S_{e}$ são os nós na face direita e esquerda do subdomínio, que terão os deslocamentos iguais ao do nó $S$. As rotações serão nulas ao longo da espessura $h$ do sub-domínio, pois não se terá a 


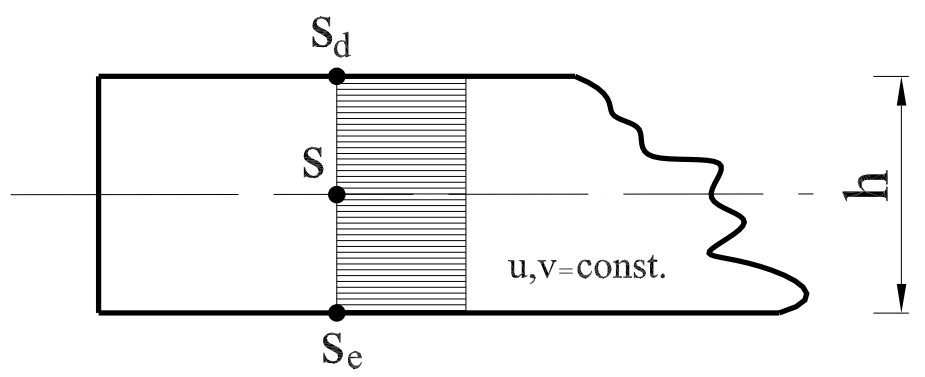

Fig. 4.2: Deslocamentos através da espessura da fibra

variação de deslocamentos através da espessura $h$ da fibra, tendo assim o sólido a rigidez a flexão zero.

A técnica utilizada consiste em escrever as equações de deslocamentos para os nós centrais da fibra $S$, como pode ser visto na figura (4.2). As integrais são definidas ao longo da interface, para os respectivos deslocamentos que são substituídos por $u_{k}(S)$, isto é, $u_{k}\left(S_{d}\right)=u_{k}\left(S_{e}\right)=u_{k}(S)$. Assim, integrando-se os elementos da interface com aproximação linear definida no eixo da sub-região fina, a matriz $H_{i j}^{*}(S)$, tem-se a contribuição dos nós da interface:

$$
H_{i j}^{*}(S)=H_{i j}^{*}\left(S_{d}\right)+H_{i j}^{*}\left(S_{e}\right)
$$

Tem-se então, a seguinte equação geral de deslocamentos para o sólido, com qualquer número de fibras $N_{f}$.

$$
c_{i j} u_{j}^{k}=\sum_{k=1}^{N_{f}} \sum_{\substack{l=0 \\ k \neq l}}^{N_{f}} \frac{-\gamma_{k}+\gamma_{l}}{\gamma_{k}} \int_{\Gamma_{k l}}\left[H_{i j}^{k *}\left(S_{e}\right)+H_{i j}^{k *}\left(S_{d}\right)\right] u_{j}^{k} d \Gamma+\sum_{k=1}^{N_{f}} \int_{\Gamma_{k 0}} G_{i j}^{k *} p_{j}^{k} d \Gamma
$$

Deve-se observar que, para os nós centrais $S$ da fibra, o valor de $c_{i j}$ é igual a $\delta_{i j}$, pois este nó é interno e pertence ao centro da fibra.

As integrais da equação (4.16), serão aproximadas no contorno e nas interfaces das sub-regiões, por elementos de contorno com funções aproximadoras lineares. 
Foi utilizada integração analítica para todos os elementos, que serão efetuadas em coordenadas locais do elemento, como mostra a figura (3.12), e posteriormente o resultado será rotacionado para o sistema global de coordenadas cartesianas.

Reunindo-se todas as contribuições das equações (3.9) de todos os elementos, pode-se escrever a equação matricial do método dos elementos de contorno:

$$
\left[\begin{array}{ll}
H_{c c} & H_{c e} \\
H_{e c} & H_{e e}
\end{array}\right]\left\{\begin{array}{l}
U_{c} \\
u_{e}
\end{array}\right\}=\left[\begin{array}{l}
G_{c c} \\
G_{e c}
\end{array}\right]\left\{P_{c}\right\}
$$

onde os índices $c$ e e referem-se a pontos do contorno e do nó central da interface, respectivamente. As matrizes $\boldsymbol{H}_{\boldsymbol{c}}$ e $\boldsymbol{H}_{\boldsymbol{e}}$ estão condensadas. Neste tipo de análise, as incógnitas da interface da fibra caem de quatro para duas por nó.

Vale lembrar que, nas integrais dos nós da interface foi utilizada a técnica singular, obtendo-se apenas como incógnitas os deslocamentos nesta região.

\subsubsection{Transformação de Domínios $2 D$ em Vigas}

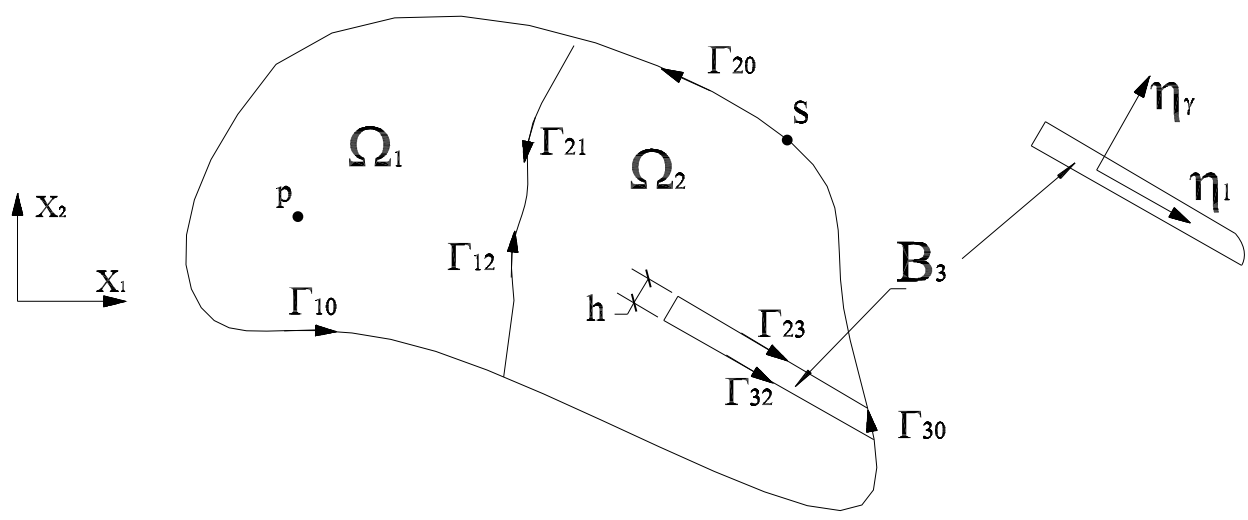

Fig. 4.3: Múltiplo domínio com inclusão de barra

Como pode ser visto no ítem anterior, a condensação de domínios muito estreitos com características de fibra impõem a este domínio uma rigidez à flexão zero, o que inviabiliza análises onde este esforço é preponderante. Nessa análise foi a adotada a hipótese de Bernoulli para viga, isto é, considera-se que o deslocamento varie 
linearmente através da espessura $h$ da inclusão, onde a seção deformada permanece reta.

Adotando-se o sistema de coordenadas locais $\left(\eta_{\gamma}, \eta_{l}\right)$ da figura (4.3) e de acordo com a figura (4.4), pode-se escrever os deslocamentos dos nós das interfaces como:

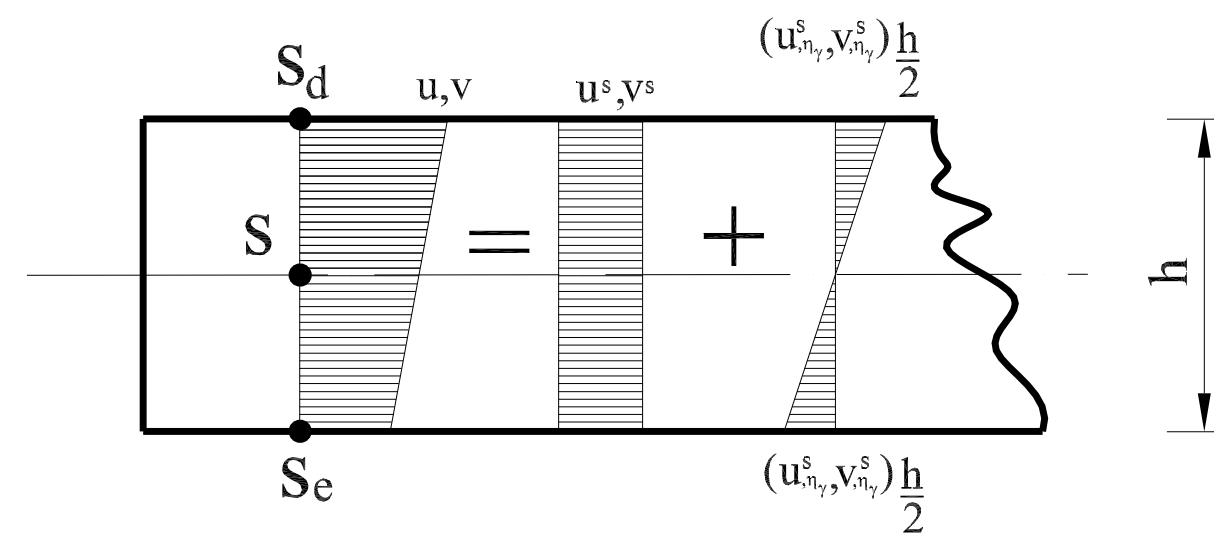

Fig. 4.4: Deslocamentos através da espessura da viga

$$
\begin{aligned}
& u_{k}^{d}=u_{k}^{s}+u_{k, \eta_{\gamma}}^{s} \frac{h}{2} \\
& u_{k}^{e}=u_{k}^{s}-u_{k, \eta_{\gamma}}^{s} \frac{h}{2}
\end{aligned}
$$

onde $u_{k, \eta_{\gamma}}^{s}$ representa a derivada do deslocamento em relação à coordenada local na direção $\eta_{\gamma}$ do eixo central da inclusão, e $u_{k}^{d}$ e $u_{k}^{e}$ são os deslocamentos dos nós da interface à direita e à esquerda do nó central.

Substituindo-se a Eq.(4.18) na Eq.(4.3) e extendendo-se a formulação para o caso de $N_{s}$ sub-regiões, tem-se:

$$
\begin{aligned}
c_{i j}^{s} u_{j}^{s}= & -\sum_{m=1}^{N_{s}} \frac{\gamma_{m}}{\gamma_{s}} \int_{\Gamma_{m 0}} H_{i j}^{*} u_{j}^{m} d \Gamma-\sum_{m=1}^{N_{\text {int }}} \frac{\gamma_{B}^{m}-\gamma_{A}^{m}}{\gamma_{s}} \int_{\Gamma_{m}} H_{i j}^{*} u_{j}^{m} d \Gamma_{m}-\sum_{m=1}^{N_{\text {int }}} \frac{\gamma_{B}^{m}-\gamma_{A}^{m}}{\gamma_{s}} \\
& \frac{h}{2} \int_{\Gamma_{m}} H_{i j}^{*} u_{j, \eta}^{m}(\vec{\eta} \cdot \vec{n}) d \Gamma_{m}-\sum_{m=1}^{N_{\text {extr }}} \frac{\gamma_{B}^{m}-\gamma_{A}^{m}}{\gamma_{s}} \int_{\Gamma_{m}} H_{i j}^{*}\left(u_{j}^{m}+u_{j, \eta}^{m} \eta\right) d \Gamma_{\text {extr }}+
\end{aligned}
$$




$$
+\int_{\Gamma} G_{i j}^{*} p_{j} d \Gamma
$$

onde $\Gamma_{\text {extr }}$ é o topo da interface da inclusão, $\gamma_{A}$ e $\gamma_{B}$ indicam os módulos de elasticidade transversais da viga e do sólido, $\eta_{s}$ e $n$ são as direções normais unitárias do centro da barra e da interface, respectivamente.

Com essa formulação, aparecem as taxas de variação de deslocamentos em relação à normal do centro da viga, podendo-se definir esta taxa como a equação de rotação no centro da barra, requerendo dessa forma que sejam escritas novas equações integrais para esta variável. Diferenciando-se a Eq.(4.19) com respeito ao eixo normal $\eta$ se obtém:

$$
\begin{array}{r}
u_{j, \eta}^{s}=-\sum_{m=1}^{N_{s}} \frac{\gamma_{m}}{\gamma_{s}} \int_{\Gamma_{m 0}} H_{i j, \eta}^{*} u_{j}^{m} d \Gamma_{m 0}-\sum_{m=1}^{N_{i n t}} \frac{\gamma_{B}^{m}-\gamma_{A}^{m}}{\gamma_{s}} \int_{\Gamma_{m}} H_{i j}^{*} u_{j, \eta}^{m} d \Gamma_{m}+ \\
-\sum_{m=1}^{N_{i n t}} \frac{\gamma_{B}^{m}-\gamma_{a}^{m}}{\gamma_{s}} \frac{h}{2} \int_{\Gamma_{m}} H_{i j, \eta}^{*} u_{j, \eta}^{m}(\vec{\eta} \cdot \vec{n}) d \Gamma_{m} \\
-\sum_{m=1}^{N_{e x t r}} \frac{\gamma_{B}^{m}-\gamma_{A}^{m}}{\gamma_{s}} \int_{\Gamma_{m}} H_{i j, \eta}^{*}\left(u_{j}^{m}+u_{j, \eta}^{m} \eta\right) d \Gamma_{e x t r}+\int_{\Gamma} G_{i j, \eta}^{*} p_{j} d \Gamma
\end{array}
$$

onde aparecem os termos:

$$
\begin{aligned}
& H_{i j, \eta}^{*}=-\frac{1}{r^{2}}\left\{(1-2 \nu) \delta_{i j}\left(\frac{\partial r}{\partial \eta} \frac{\partial r}{\partial n}-\frac{\partial \eta}{\partial n}\right)+2\left[\left(4 \frac{\partial r}{\partial \eta} r_{i} r_{j}-\eta_{i} r_{j}-\eta_{j} r_{i}\right)\right.\right. \\
& \left.\left.\frac{\partial r}{\partial n}-r_{i} r_{j} \frac{\partial \eta}{\partial n}\right]-(1-2 \nu)\left[\left(2 r_{i} \frac{\partial r}{\partial \eta}-\eta_{i}\right) n_{j}-\left(2 r_{j} \frac{\partial r}{\partial \eta}-\eta_{j}\right) n_{i}\right]\right\} \\
& G_{i j, \eta}^{*}=-(3-4 \nu) \delta_{i j} \frac{\partial r}{\partial \eta}+\eta_{i} r_{j}+\eta_{j} r_{i}-2 r_{i} r_{j} \frac{\partial r}{\partial \eta}
\end{aligned}
$$


As integrais das equações (4.19) e (4.20), serão aproximadas no contorno e nas interfaces das sub-regiões por elementos de contorno, com funções aproximadoras lineares. Foi utilizada integração analítica para todos os elementos, sendo que as mesmas serão efetuadas em coordenadas locais do elemento a ser integrado, como mostra a figura (3.12), e posteriormente o resultado será rotacionado para o sistema global de coordenadas cartesianas.

Reunindo-se todas as contribuições das equações (4.19) e (4.20), de todos os elementos, pode-se escrever a equação matricial do método dos elementos de contorno:

$$
\left[\begin{array}{lll}
H_{c c} & H_{c e} & H_{c r}^{\prime} \\
H_{e c} & H_{e e} & H_{e r}^{\prime} \\
H_{e c} & H_{e e} & H_{e r}^{\prime}
\end{array}\right]\left\{\begin{array}{l}
U_{c} \\
u_{e} \\
u_{r}^{\prime}
\end{array}\right\}=\left[\begin{array}{l}
G_{c c} \\
G_{e c} \\
G_{r c}^{\prime}
\end{array}\right]\left\{P_{c}\right\}
$$

onde os termos escritos com ', se referem às soluções fundamentais de rotação em relação ao eixo central da viga. Os termos escritos com o sub-índice $r$ se referem as incógnitas de rotação da viga.

Pode-se escrever a equação de tensão de pontos internos, bastando para isso derivar e utilizar a lei de Hooke na equação (4.19).

\subsection{Análise numérica}

\subsubsection{Estaca em Meio Contínuo}

A figura (4.5) mostra uma estaca imersa em um sólido que está representando um meio-contínuo.

Os módulos de elasticidade admitidos neste problema são $E_{s}=2,1 \times 10^{10} \mathrm{~N} / \mathrm{m}^{2}$ para o meio contínuo e $E_{p}=2,1 \times 10^{11} \mathrm{~N} / \mathrm{m}^{2}$ para a estaca, e o coeficiente de Poisson $\nu=0,2$ para as duas sub-regiões. Foi utilizada, na análise 2D, as hipóteses do Estado Plano de Deformações.

As condições de contorno definidas no problema são restrições a deslocamentos verticais e horizontais nas laterais e na base do meio-contínuo. A estaca será 


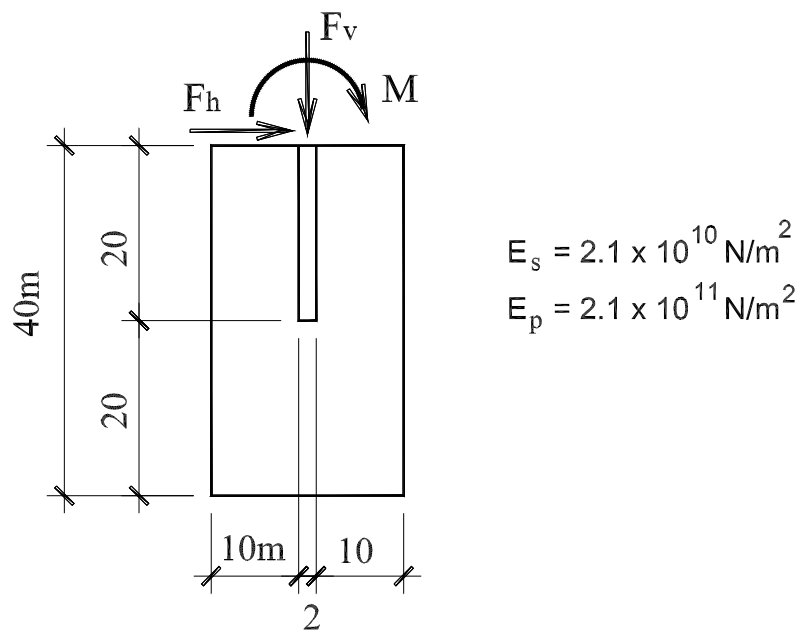

Fig. 4.5: Estaca em meio contínuo

carregada na sua extremidade superior com os seguintes carregamentos, os quais serão analisados separadamente: uma carga horizontal $F_{h}=40 k N$, carga vertical de $F_{v}=160 k N$ e momento $M=9,975 k N m$.

O problema será resolvido usando três modelos distintos. Nos dois primeiros serão utilizados sub-regiões 2D, isto é, barra e sólido serão discretizados com subregiões com aproximação linear de forças e deslocamentos no contorno e na interface.

No primeiro modelo serão empregadas apenas equações de deslocamentos no contorno e na interface (4.6). Portanto, tem-se apenas incógnitas de deslocamentos na interface. No segundo serão empregadas equações hipersingulares (4.13) na interface e singulares (4.6) no contorno, obtendo-se apenas incógnitas de força de superfície na interface.

Na terceira alternativa utilizou-se o modelo de viga obtido com a aproximação linear de deslocamentos na interface, portanto emprega-se a equação (4.19) e (4.20) para a viga, e a (4.6) para o contorno.

A figura (4.6), mostra a discretização adotada, bem como a notação adotada nos modelos analisados:

O contorno externo do solo é discretizado com 160 nós em cada face, enquanto que, nas laterais da interface, foram adotados 300 nós em cada face. No topo e na ponta da estaca foram adotados numa primeira análise, uma discretização mais pobre, de dois nós em cada face e, numa malha mais refinada, 40 nós em cada face. A 


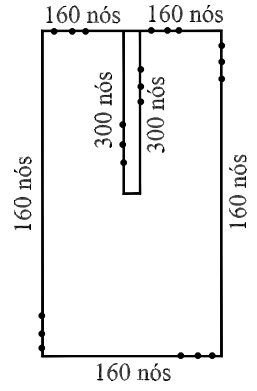

a)

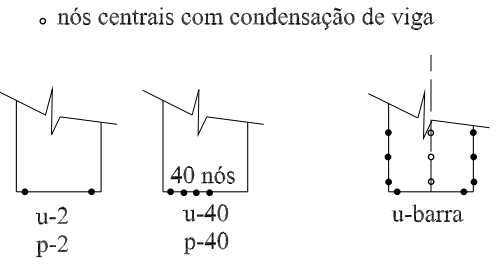

b) c)

Fig. 4.6: a) Discretização do meio contínuo b) Discretização da base da estaca c) Discretização da base da estaca e posição dos nós da formulação de barra.

formulação de barra admite apenas 2 nós no topo e na base da estaca. Os elementos utilizados possuem aproximação linear de geometria e também de deslocamentos e forças de superfície, enquanto que o comprimento dos elementos em cada face é igual. Os resultados são comparados com o modelo de combinação BEM/FEM (Coda ; Venturini, 1999), onde a estaca foi discretizada em 100 elementos finitos de barra, enquanto que a face externa do solo foi discretizado por 50 elementos de contorno com aproximação quadrática. A seguir, estão os resultados em termos de deslocamentos na linha central do fuste, e forças na interface solo/estaca.

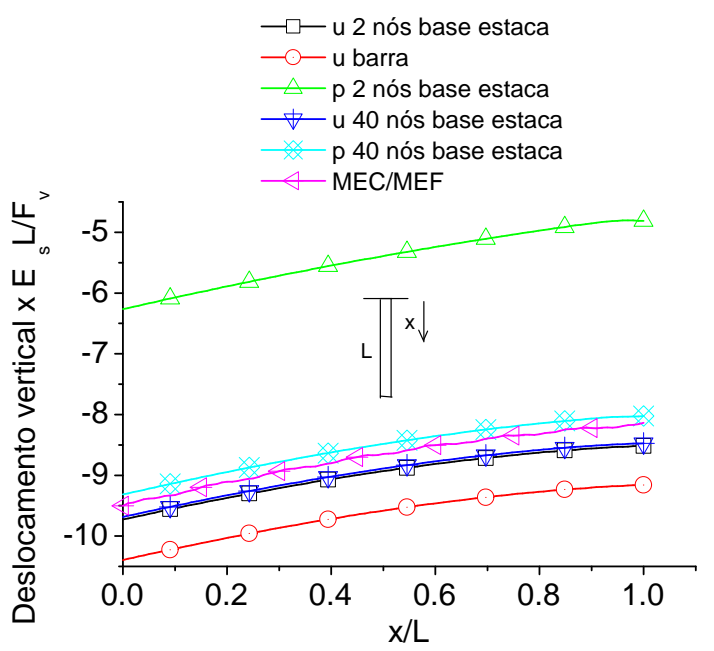

Fig. 4.7: Deslocamento vertical ao longo do fuste devido à força $F_{v}$ 


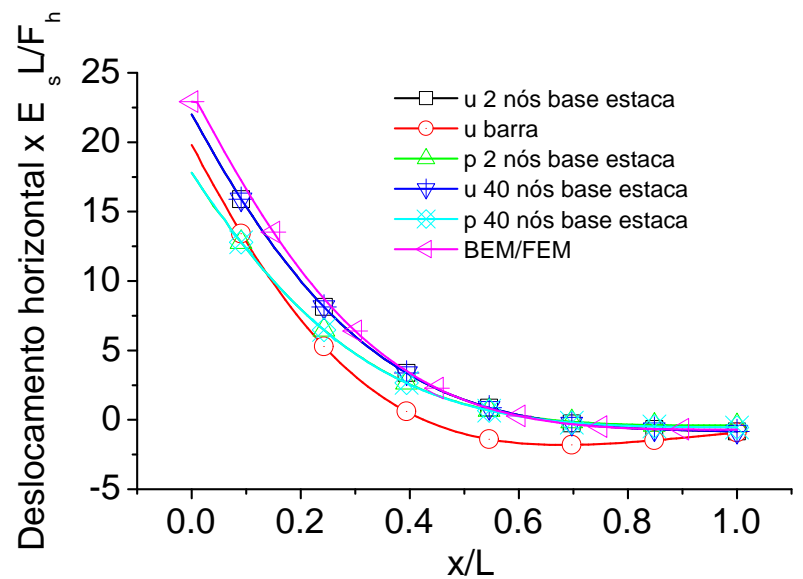

Fig. 4.8: Deslocamento horizontal ao longo do fuste devido à força $F_{h}$

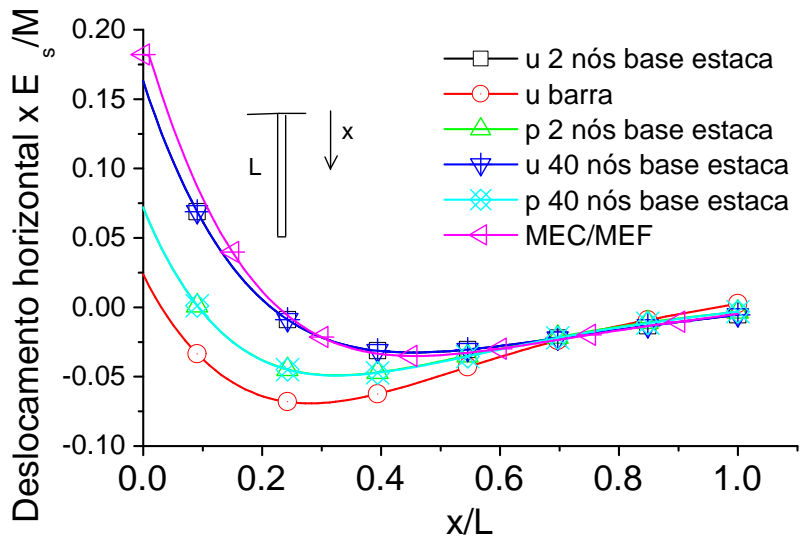

Fig. 4.9: Deslocamento horizontal ao longo do fuste devido ao momento $M$ 


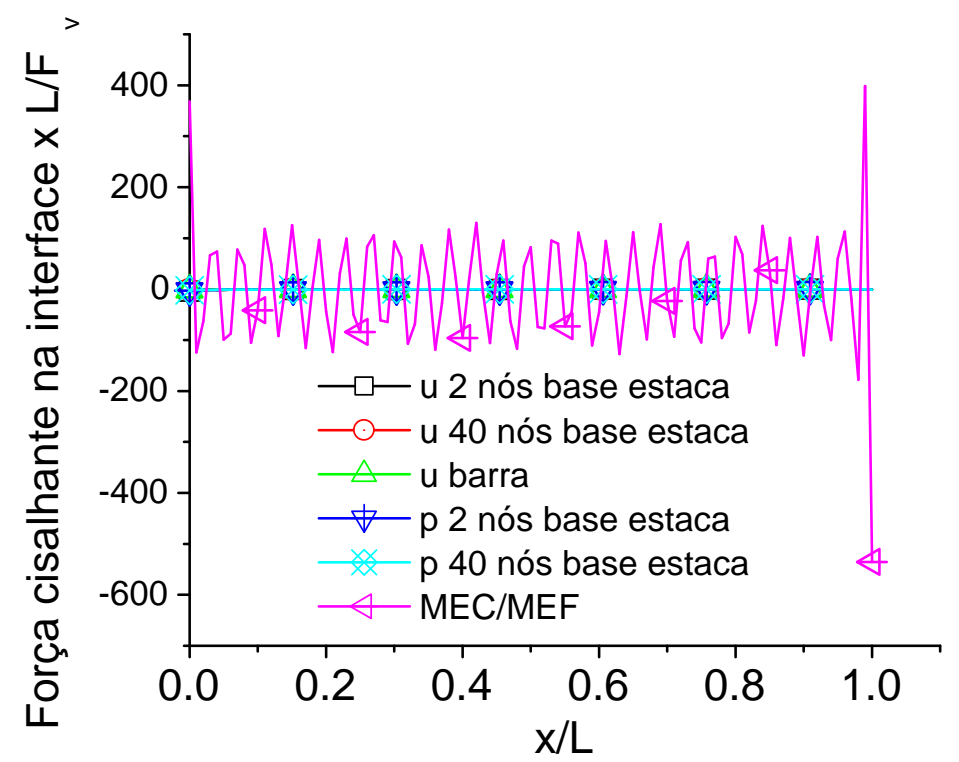

Fig. 4.10: Força tangencial à interface devida à força $F_{v}$

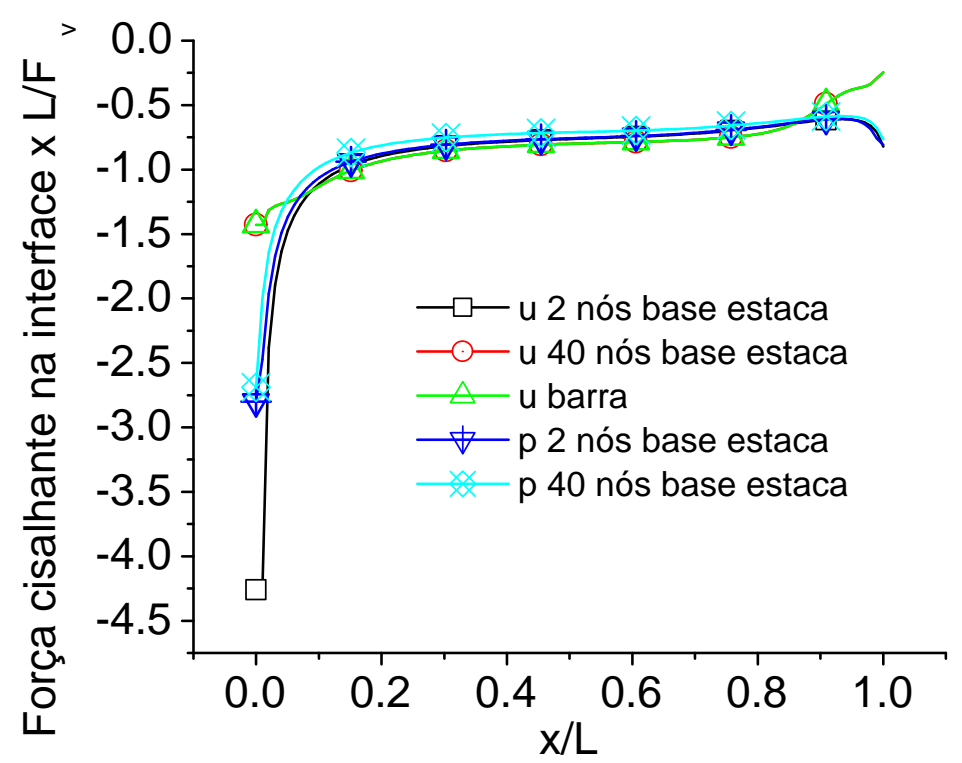

Fig. 4.11: Força tangencial à interface devida à força $F_{v}$, apenas MEC 


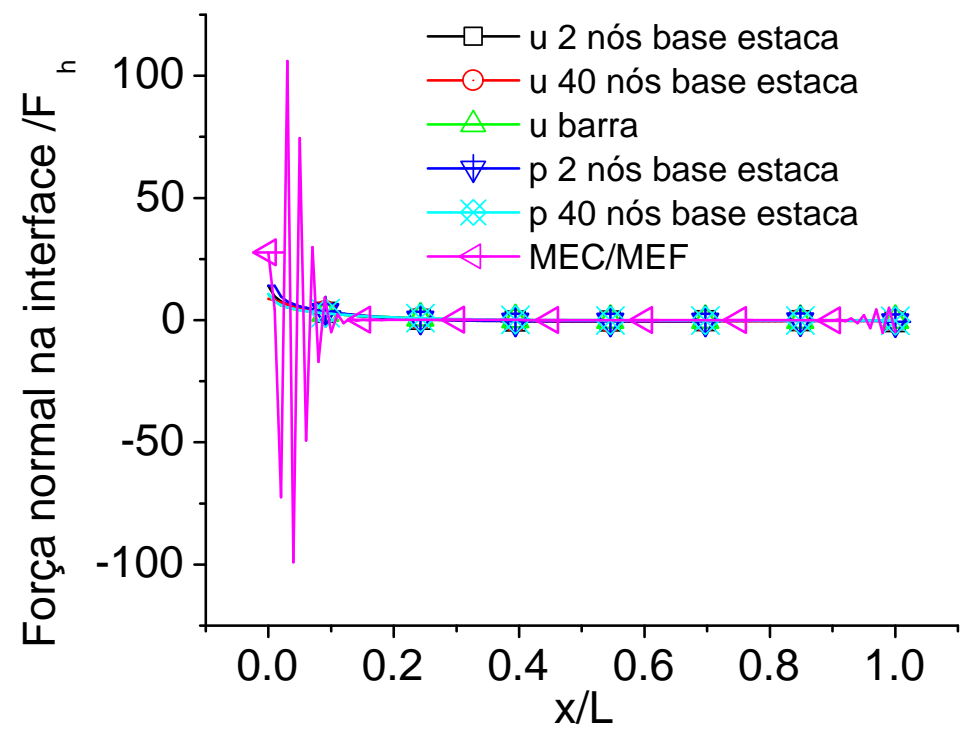

Fig. 4.12: Força normal à interface devida à força $F_{h}$

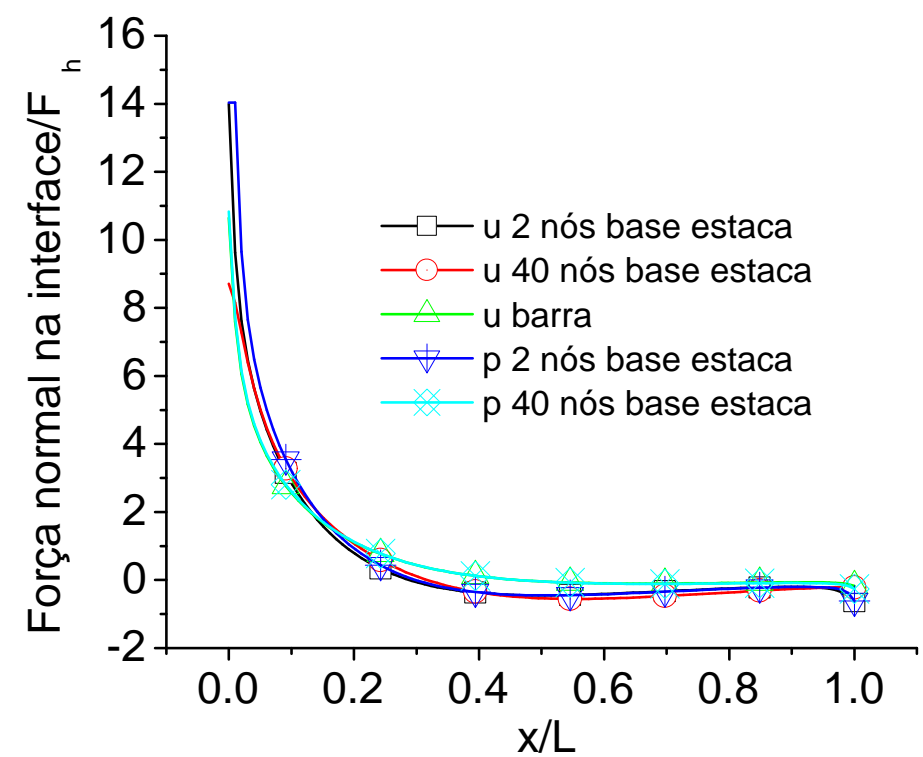

Fig. 4.13: Força normal à interface devida à força $F_{h}$, apenas MEC 


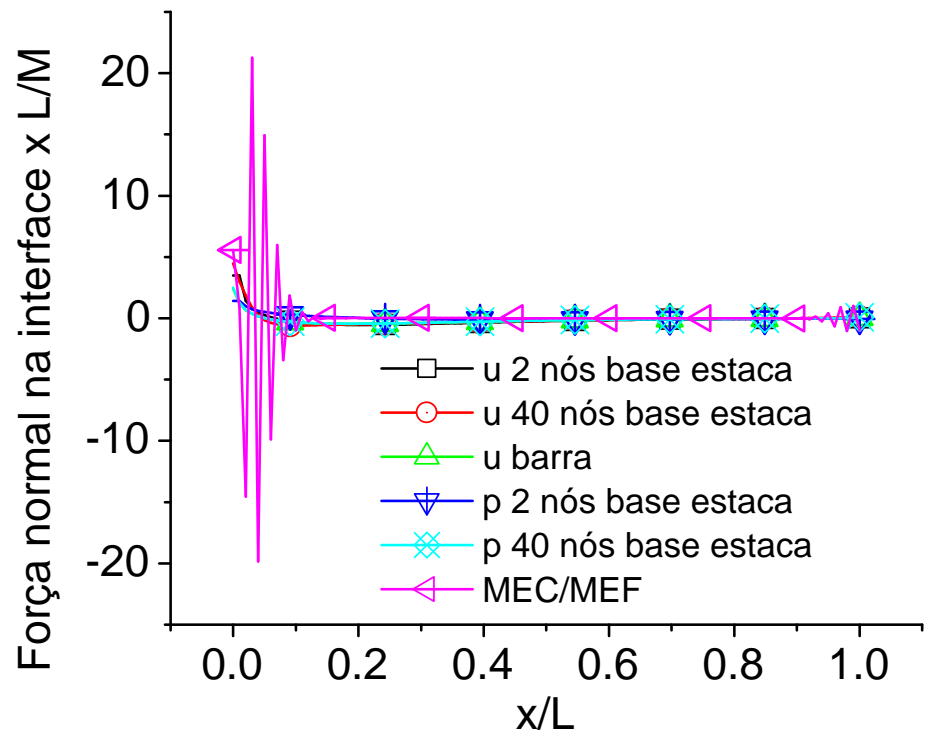

Fig. 4.14: Força normal à interface devida ao momento $M$

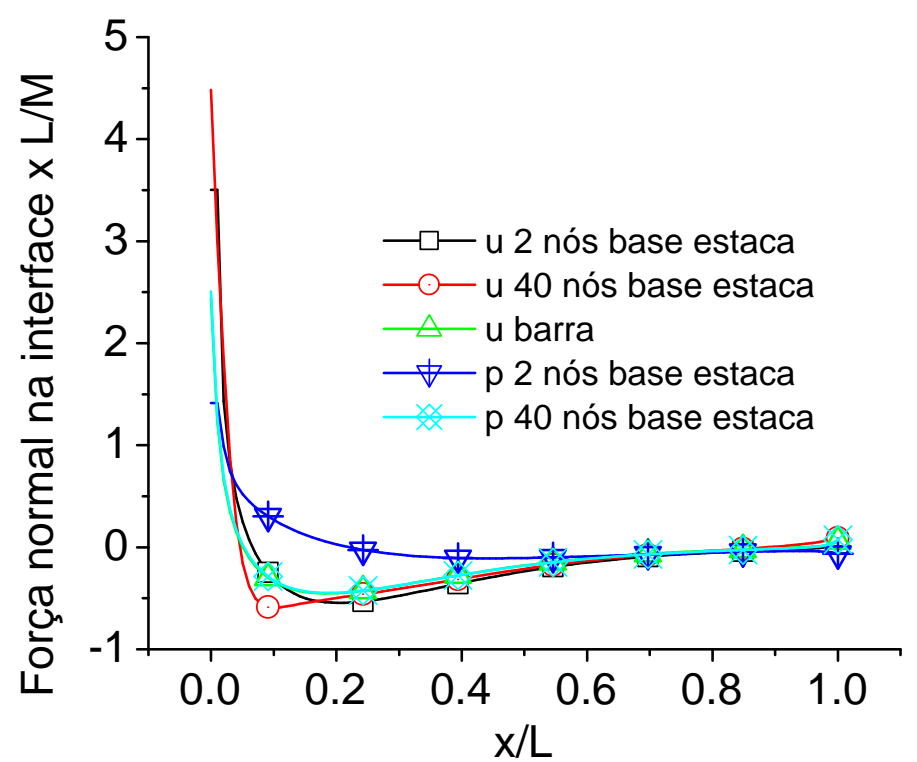

Fig. 4.15: Força normal à interface devida ao momento $M$, apenas MEC 
Os resultados deste exemplo no geral se apresentam com boa precisão, acompanhando o comportamento físico esperado do sólido em um meio contínuo. Nota-se neste caso que a formulação hipersingular apresenta os piores resultados, sendo que somente a partir de uma maior discretização da malha, os resultados acompanham os demais. Isso se deve ao fato da formulação apresentar fortes singularidades no contorno, principalmente na ponta da estaca, que é uma região onde há canto.

Os melhores resultados são obtidos pela formulação singular, pois nota-se que não há praticamente nenhuma diferença entre a malha mais pobre e a malha mais refinada. Conclui-se que a formulação singular é bem estável e apresenta matrizes bem condicionadas.

Na técnica de condensação de viga, os resultados destoaram dos demais, pois tal formulação leva em conta as hipóteses de Bernoulli, tornando o sólido mais rígido e, como o solo possui rigidez menor, há um maior deslocamento da estaca. Contribui para isso também o fato da espessura da inclusão não ser tão esbelta em relação ao domínio principal, o que potencializa o efeito das hipóteses de Bernoulli.

Foi notada na análise de tensões, utilizando-se o MEC/MEF, uma grande oscilação na região da cabeça da estaca. Isso se deve ao fato do elemento finito utilizado buscar o equilíbrio isoladamente. Mas utilizando-se técnicas de regularização, como mínimo quadrado, tal problema é facilmente contornado (Botta, 2003).

\subsubsection{Viga de concreto armado}

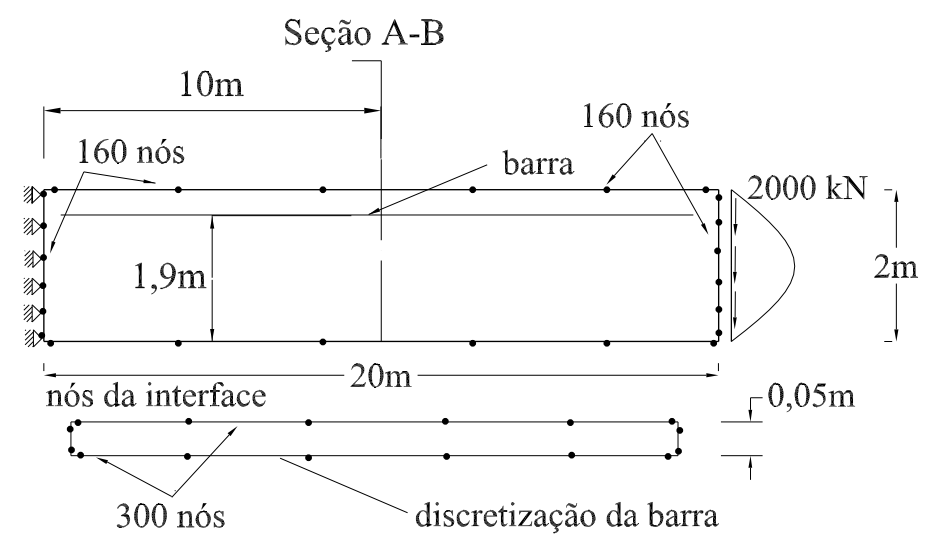

Fig. 4.16: Sólido com inclusão. Características geométricas e discretização 
A figura (4.16) mostra um sólido 2D, com uma inclusão simulando uma barra de material diferente no comportamento, de uma viga de concreto armado, com comportamento puramente elástico. A figura mostra ainda as características geométricas da estrutura, bem como a discretização adotada em elementos de contorno. A relação elástica entre a inclusão e o domínio principal é de $E_{i} / E_{s}=10$, e ambas possuem coeficiente de Poisson $\nu=0.25$, com as hipóteses do estado plano de tensão.

Serão utilizadas nesse exemplo: - a formulação singular no contorno e na interface, denominada de singular. - a formulação singular no contorno e hiper singular na interface, denominada de hiper singular. - a formulação de condensação de domínio em viga, denominada de u barra. As figuras (4.17) e (4.18) mostram o desenvolvimento da tensão normal e através da seção $A-B$ :

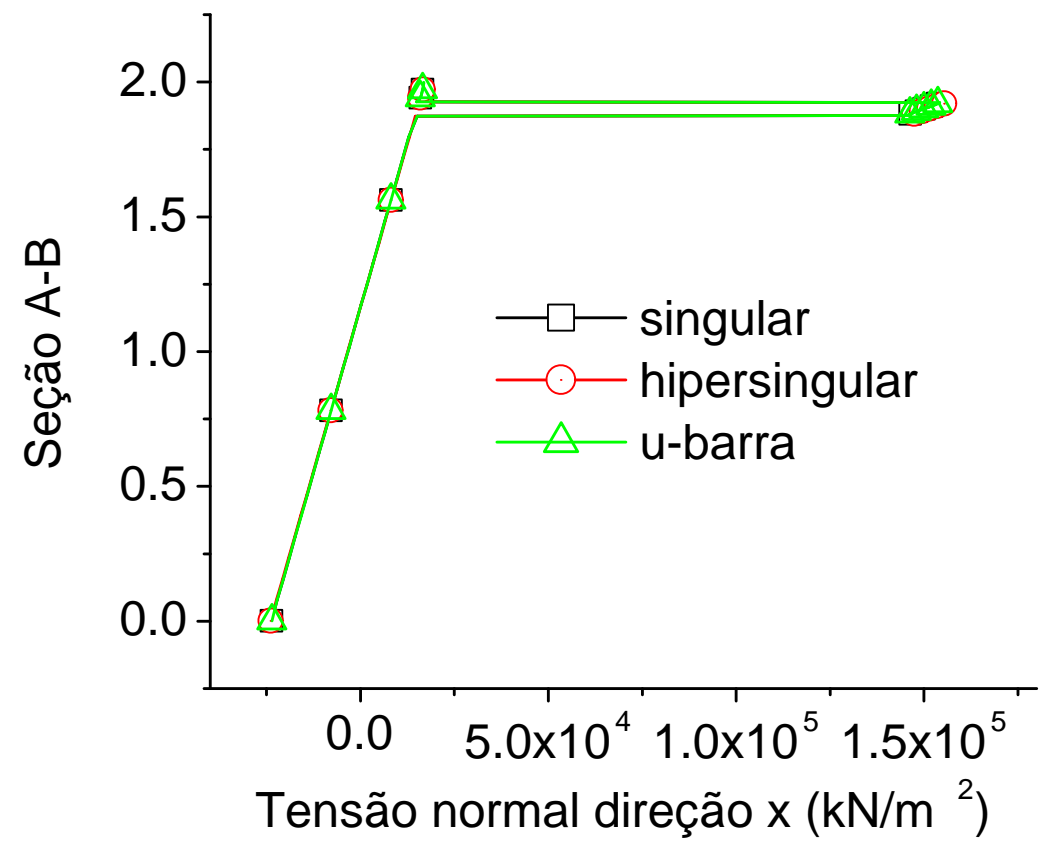

Fig. 4.17: Tensão normal atuante através da seção A-B

Tem-se a seguir, os resultados em termos de tensão cisalhante atuante através da seção A-B: 


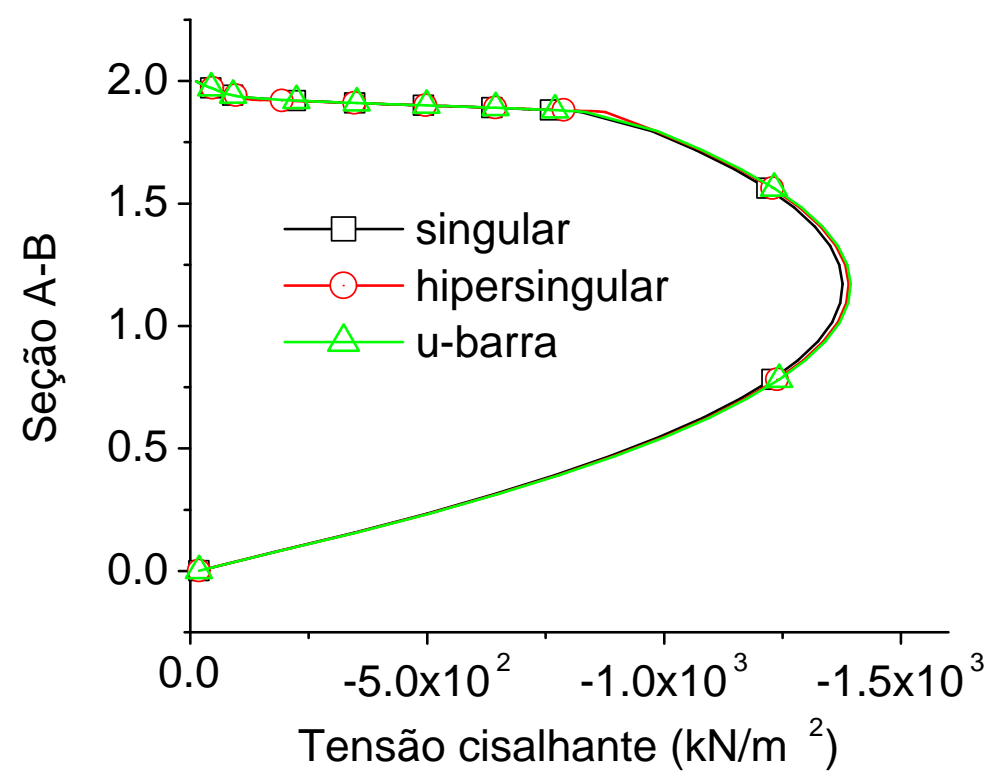

Fig. 4.18: Tensão cisalhante atuante através da seção A-B

A seguir, têm-se os resultados em termos de esforços na seção transversal, comparados com os resultados exatos da teoria de viga.

Tab. 4.1: Esforços atuantes através da seção transversal A-B

\begin{tabular}{|c|c|c|c|c|}
\hline Análise & $\begin{array}{c}\text { Esforço Cortante } \\
(k N)\end{array}$ & $\begin{array}{c}\text { Erro } \\
\%\end{array}$ & $\begin{array}{c}\text { Momento Fletor } \\
(k N m)\end{array}$ & $\begin{array}{c}\text { Erro } \\
\%\end{array}$ \\
\hline Teoria de viga & 2000.0000 & - & 20000.00 & - \\
\hline $\mathbf{u}$ & 1952.7484 & 2.3626 & 20123.9377 & 0.6197 \\
\hline $\mathbf{u}$ barra & 1951.7494 & 2.4125 & 20116.1347 & 0.5807 \\
\hline $\mathbf{p}$ & 1972.0286 & 1.3986 & 20303.8418 & 1.5192 \\
\hline
\end{tabular}

Este exemplo mostra a boa precisão das técnicas utilizadas, que são as formulações singulares, hipersingular e condensação em viga, que são comparados com resultados da teoria exata de vigas. Nota-se, que neste caso, como a inclusão é muito fina, de certa forma anula-se os efeitos das hipóteses de Bernoulli, e com isso os resultados da análise da condensação em viga se aproxima dos resultados da 
elasticidade plana.

\subsubsection{Fratura Elástica - Sólido com fraturamento}

O terceiro exemplo mostra a capacidade da formulação de inclusão por subdomínios para simular o comportamento de uma região sujeita ao fraturamento. Nesse caso, é adotado um módulo de elasticidade com valor muito próximo de zero para a inclusão, que por ser muito fina, se assemelha a uma fratura. Nota-se que esta formulação difere da formulação tradicional de fratura, pois aqui ela possui uma abertura inicial, sendo que, os resultados perto da ponta da fratura podem se apresentar diferentes dos obtidos pela solução teórica, que considera a fratura inicial sem a separação de suas faces.

A figura a seguir, mostra um sólido com uma fratura com inclinação $\alpha$, que será analisada para valores de inclinação variando de $\alpha=0$ até $\alpha=30^{\circ}$. As excentricidades $e_{x}$ e $e_{y}$ tem valores de $L / 4$ e a espessura adotada na região de fraturamento é de $w_{f}=4 L \times 10^{-3}$.

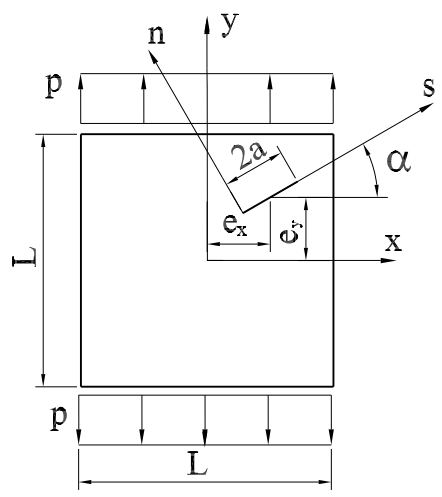

Fig. 4.19: Sólido com fratura

Os resultados em termos de tensão na região logo após a ponta da fratura, serão comparados com análises feitas com o Método de Colocação de Contorno (BCM) (Yuanhan, 1991). O contorno externo foi discretizado em 320 elementos com aproximação linear, enquanto que a fratura foi discretizada com 600 elementos. As figuras a seguir mostram as tensões normais e cisalhantes logo adiante à ponta da fratura. 


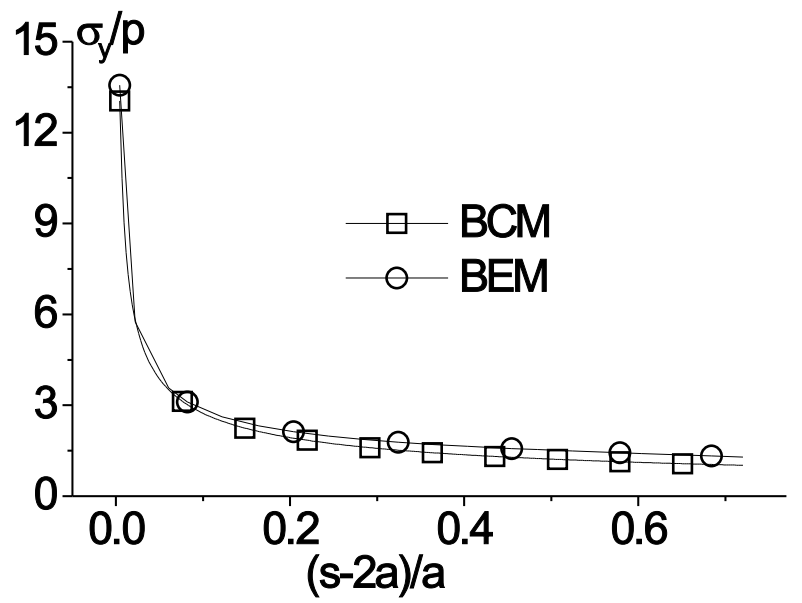

Fig. 4.20: Tensão $\sigma_{x}$ após a ponta da fratura para $\alpha=0^{\circ}$

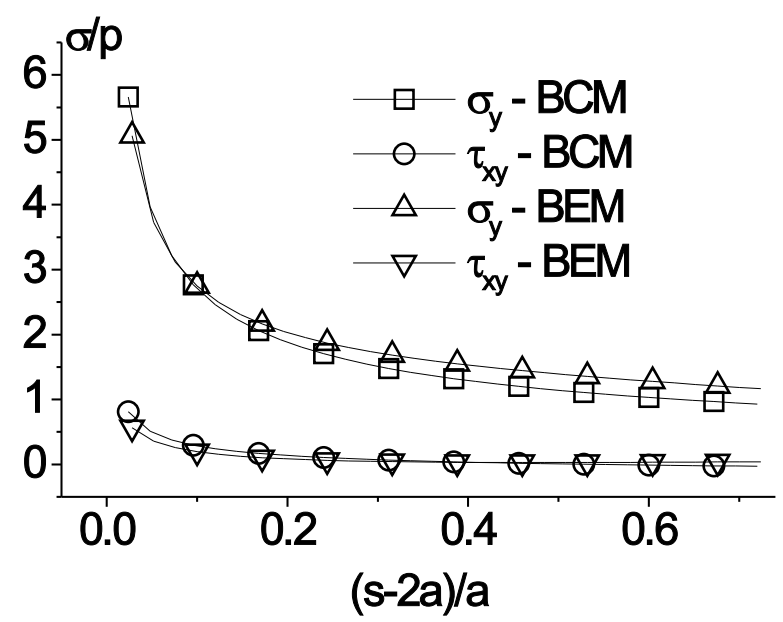

Fig. 4.21: Tensão $\sigma_{x}$ e $\tau_{x y}$ após a ponta da fratura para $\alpha=30^{\circ}$

Neste exemplo fica demonstrado a precisão das integrais analíticas para se discretizar domínios tão esbeltos. Os resultados na ponta da fratura foram satisfatórios, lembrando-se que neste caso a fratura é uma região discreta, ao contrário da análise feita pelo BMC, o qual utilizou uma linha de fratura. Fica demonstrado que a formulação pode analisar fratura nas mais diversas posições. 


\subsubsection{Domínio com inclusão de rigidez infinita}

O presente exemplo mostra um domínio retangular contendo uma inclusão com dimensões da mesma ordem de grandeza, como mostra a figura (4.22).

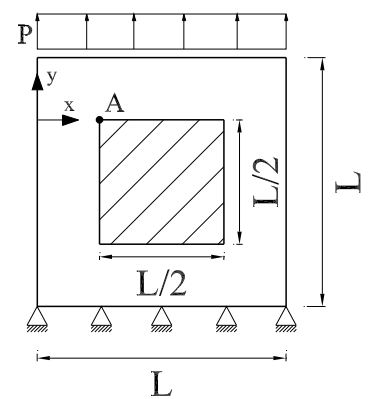

Fig. 4.22: Domínio quadrado com inclusão e condições de contorno

O objetivo deste exemplo é a análise das tensões atuantes nos pontos críticos da interface, ou seja, nos cantos, analisados neste exemplo no ponto $A$.

Foi utilizada, nesse exemplo, a formulação singular no contorno e na interface (4.6), pois a mesma elimina as equações de forças de superfície da interface do sistema de equações de equilíbrio. Os resultados obtidos pela formulação hipersingular (4.13), apresentam grandes oscilações nesta região para valores de rigidez da inclusão muito maiores que a do domínio principal. Isso se deve ao fato da formulação hipersingular apresentar, nas regiões perto dos cantos, valores muito altos, o que acarreta uma matriz algébrica mal condicionada.

As características de rigidez da inclusão, $E_{i}$, variam segundo a relação $E_{i}=$ $E \cdot 10^{n}$ onde $E$ é o módulo de elasticidade longitudinal do domínio principal. Foi iniciada uma análise com $n=2$, até um valor de $n$ onde se observe uma convergência e estabilidade das respostas. O coeficiente de Poisson adotado para ambos os domínios foi de $\nu=0,25$. Foi feita uma comparação utilizando-se o Método dos Elementos Finitos, utilizando o programa ANSYS (versão 5.7), onde os domínios foram discretizados em 6400 elementos retangulares (Shell 47) de elasticidade plana.

$\mathrm{Na}$ análise, utilizando-se o Método dos Elementos de Contorno, foram adotados 320 nós em todo o contorno do exemplo, divididos igualmente entre as faces. A figura (4.23) mostra os resultados de deslocamentos ao longo do eixo $x$, como mostra 
a figura (4.22), que atinge o canto entre os domínios no valor de $x / l=0,25$. Foi feita uma comparação entre a formulação singular deste capítulo com as respostas obtidas pelo programa ANSYS. Como pode ser visto, nesta análise, para $n=4$ os resultados se estabilizam e a inclusão já pode ser chamada de "corpo-rígido".

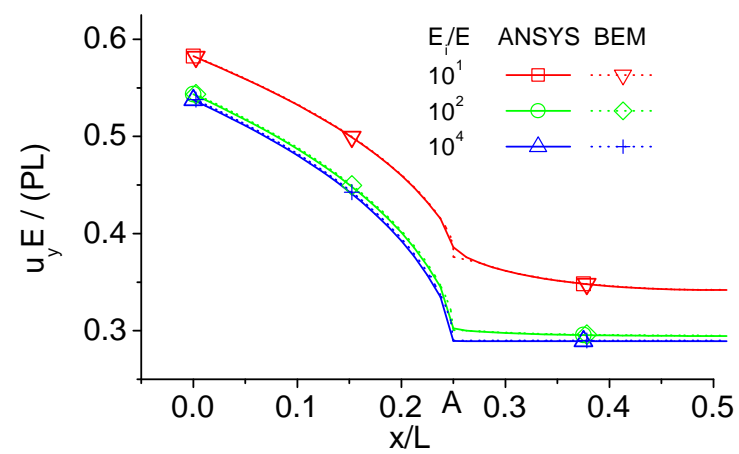

Fig. 4.23: Deslocamento vertical ao longo do eixo $x$ com diferentes módulos de rigidez longitudinal da inclusão

Uma segunda análise diz respeito ao desenvolvimento das tensões $\sigma_{y}$ ao longo do eixo $x$, figura (4.24), passando pelo ponto $A$ de canto entre os subdomínios. Observa-se, neste caso, um desenvolvimento suave das curvas exceto um valor acentuado no ponto A.

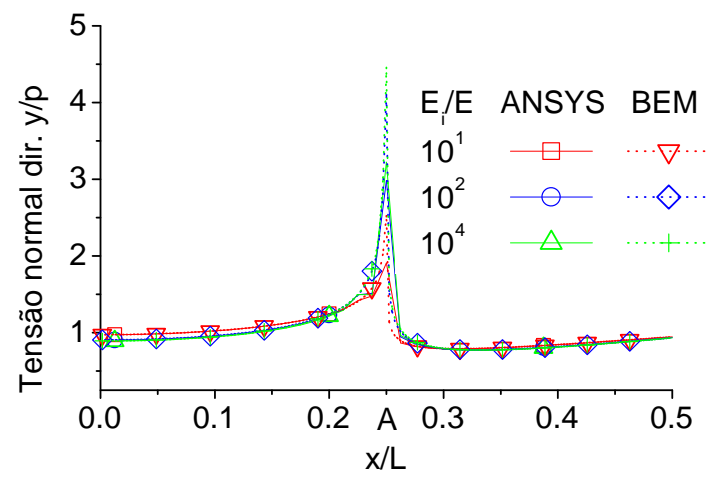

Fig. 4.24: $\sigma_{y} / p$ ao longo da abscissa $x$. 
Foram utilizados pontos de colocação no modelo de elementos de contorno a uma distância de $L / 2 \cdot 10^{-9}$, o que mostra a precisão das integrais analíticas utilizadas. Foi observada uma diferença entre os valores de $\sigma_{y}$ dos pontos mais próximos do canto de $19,8 \%, 32,4 \%$ e $34,5 \%$ para $E_{i} / E=10,10^{2}$ e $10^{4}$ respectivamente, utilizando-se o Método dos Elementos de Contorno, enquanto que na análise utilizando-se o programa ANSYS foi observada uma diferença de 76,6\% para $E_{1} / E=10^{4}$. No gráfico (4.25), é demonstrado o desenvolvimento das tensões $\sigma_{x}$ ao longo de pontos situados próximos a interface nas proximidades do canto $\mathrm{A}$ ,tanto do domínio principal quanto da inclusão.

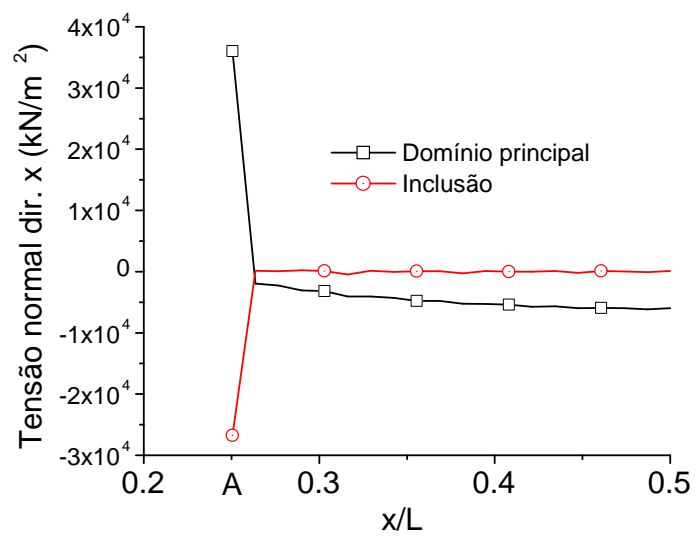

Fig. 4.25: Tensão $\sigma_{x}$ ao longo do domínio principal e da inclusão

Nesse exemplo fica comprovada a eficácia da formulação singular na análise de sólidos com inclusões com rigidezes consideradas infinitas. Foram feitas comparação com o programa ANSYS, que possui grande confiabilidade mundial, e os resultados se aproximaram bastante. $\mathrm{Na}$ análise de canto, a formulação singular apresentou melhores resultados no que se refere aos gradientes de tensão, que foram mais suaves que os apresentados pelo ANSYS. Na figura (4.25) ficou demonstrado que o desenvolvimento das tensões, tanto no domínio principal quanto na inclusão, tendem ao infinito, mas em sentido contrário. 


\subsubsection{Domínio enrijecido por fibras}

O exemplo seguinte consiste de um domínio retangular reforçado por quatro inclusões com características físicas de fibras, utilizando-se para isto a técnica de condensação de domínios, como mostra a figura (4.26).

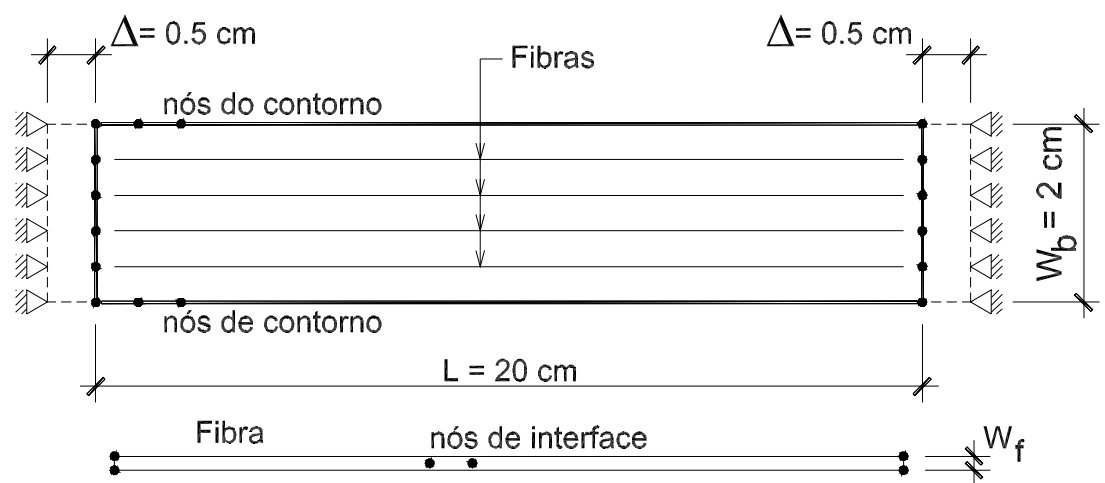

Fig. 4.26: Domínio com inclusão de fibras. Dimensões e discretização.

O módulo de elasticidade longitudinal adotado no domínio principal é $E_{1}=$ $2000 \mathrm{kN} / \mathrm{cm}^{2}$ sendo adotado o valor do coeficiente de Poisson, $\nu=0$, para ambos domínios. A rigidez do domínio principal é dada pela fórmula $E_{1} A_{1}=4000 k N$ onde $A_{1}$ é a área da seção transversal, enquanto que a rigidez das quatro fibras é dada por $4 A_{2} E_{2}$ onde $A_{2}$ é a área da seção transversal de cada fibra. Pode-se relacionar a rigidez das fibras com a rigidez do domínio retangular pela relação $4 A_{2} E_{2}=\alpha E_{1} A_{1}$. Neste exemplo foi adotado $\alpha=4$, ou seja, a rigidez das fibras é quatro vezes maior que a rigidez do sólido. A espessura das fibras é de $10^{-4} \mathrm{~cm}$, onde se tem a relação entre a espessura da fibra e a espessura da viga de $w_{f} / w_{b}=5 \times 10^{-5}$. Foi adotada uma malha de 25 nós no contorno em cada face e uma malha fina de 365 nós no centro de cada fibra. Para se comprovar a eficiência das formulações de condensação em fibra e das integrais analíticas, foi adotada a espessura das fibras variando de $10^{-1} \mathrm{~cm} \geq w_{f} \leq 10^{-4} \mathrm{~cm}$. O sólido, como mostra a figura (4.26), está sujeito a uma ação de deslocamentos lineares de $0,5 \mathrm{~cm}$

Os resultados são comparados com análise feita utilizando-se o modelo de combinação MEC/MEF (Coda ; Venturini, 1999), onde o sólido foi discretizado 
em elementos de contorno utilizando-se 48 nós com aproximação quadrática e 600 elementos finitos de barra na interface.

A figura (4.27) mostra a tensão normal às fibras na vizinhança da interface entre o domínio principal e a fibra, sendo Dist a distância do ponto fonte à interface da fibra. Tem-se para a relação Dist $/ w_{f} \leq 10^{-2}$, valores das integrais numéricas garantindo boa precisão, ao passo que para as integrais analíticas a mesma precisão é garantida para valores até $\operatorname{Dist} / w_{f} \leq 10^{-4}$.

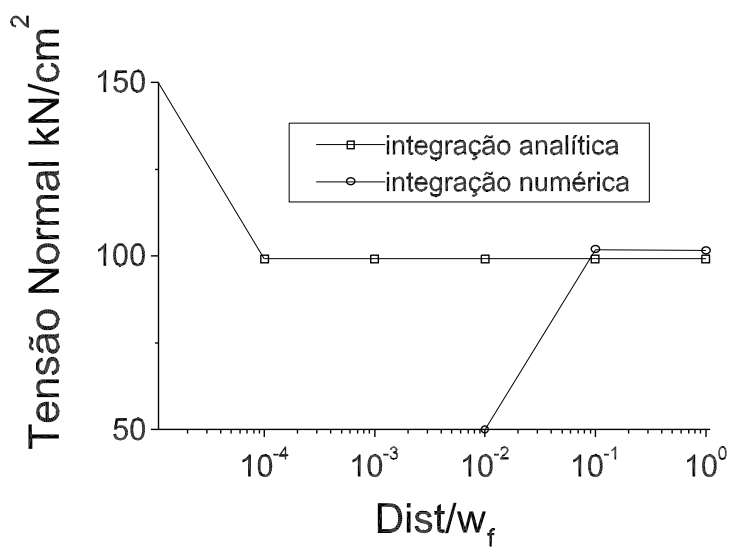

Fig. 4.27: Tensão normal nas proximidades da fibra.

A figura (4.28) mostra o deslocamento longitudinal no eixo central do domínio principal. Foi feita uma análise, considerando-se a relação espessura da fibra e espessura da barra, $w_{f} / w_{b}$, variando de $5 \times 10^{-2}$ a $5 \times 10^{-5}$, mas mantendo a mesma rigidez do conjunto, ou seja, aumentando-se proporcionalmente a rigidez das fibras.

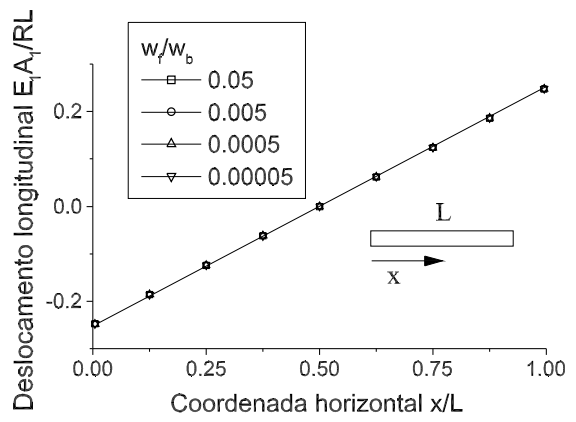

Fig. 4.28: Deslocamentos para vários valores de $w_{f} / w_{b}$ 
A figura (4.29) mostra a força tangencial à inteface da fibra na posição inferior da barra, utilizando-se as mesmas hipóteses da análise anterior.

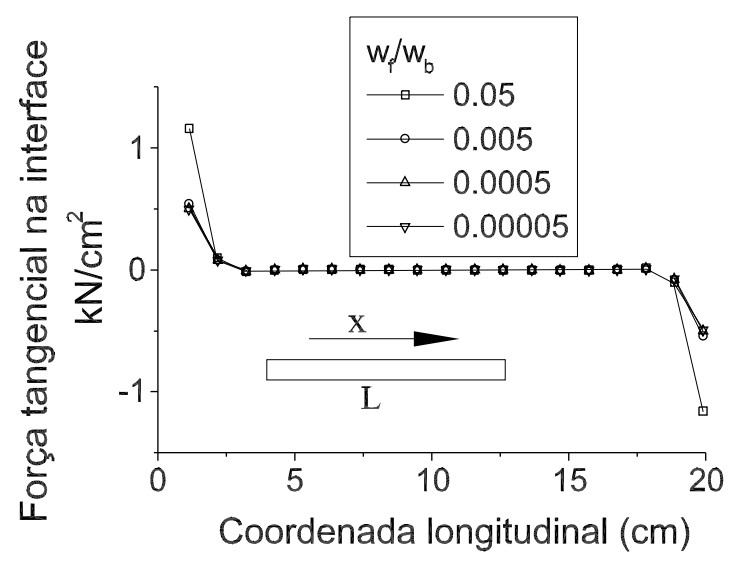

Fig. 4.29: Força tangencial na fibra inferior para vários valores de $w_{f} / w_{b}$

Como mostra a figura (4.30), foi feita uma comparação com a combinação $\mathrm{MEC} / \mathrm{MEF}$, onde analisa-se o deslocamento longitudinal ao longo do eixo central do domínio principal.

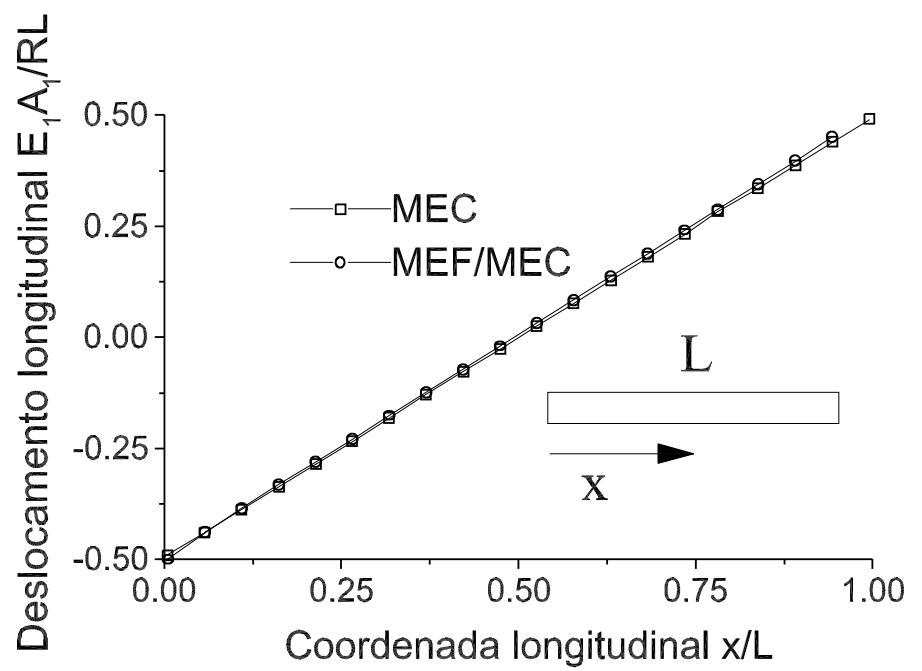

Fig. 4.30: Deslocamento longitudinal com $\alpha=1.0$ e $w_{f} / w_{b}=005$

Nos gráficos seguintes, são mostradas as variações da força tangencial ao longo 
da espessura da fibra inferior contida no domínio 2D, para $\alpha=1,0$ e para a rigidez do conjunto variando de $\alpha=1$ a $\alpha=4$.

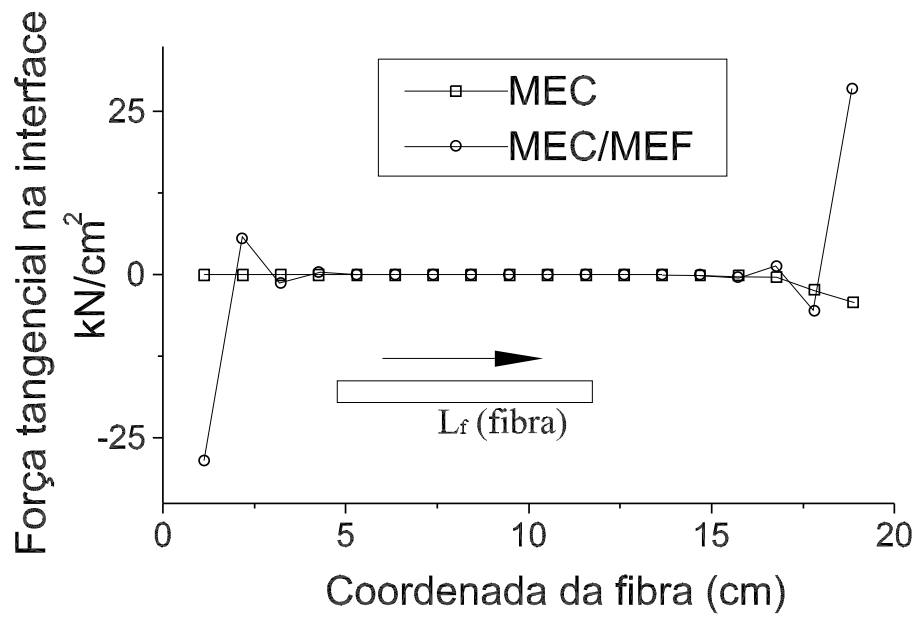

Fig. 4.31: Força tangencial na interface com $\alpha=1.0$ e $w_{f} / w_{b}=005$

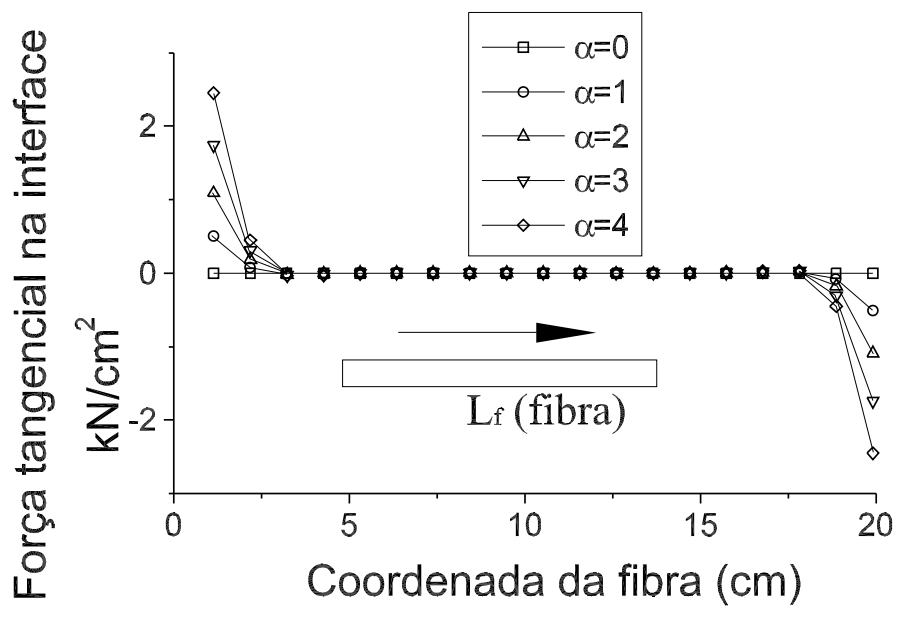

Fig. 4.32: Força tangencial na interface com várias rigidezes

Neste exemplo fica comprovada a eficácia da solução singular e da condensação em fibras para inclusões muito esbeltas, e também com as várias rigidezes das mesmas. Os resultados foram comparados com a técnica MEC/MEF, onde ambos convergiram, exceto nas regiões de ponta das inclusões, onde a análise MEC/MEF 
apresenta grandes oscilações nas análises de tensões nesta região. Ficou comprovado, como mostra a figura (4.27), a eficácia da integração analítica em comparação com o processo de integração numérica. 


\section{ANÁLISE NÃO-LINEAR}

\subsection{Introdução}

No presente capítulo, será apresentado a análise não-linear realizada neste trabalho. Inicialmente, será apresentada uma breve introdução da teoria que emprega campo inicial de tensões, indispensável na análise não-linear via Método dos Elementos de Contorno, que se adapta a uma gama muito grande de modelos constitutivos. Na mesma seção, discutem-se as equações algébricas e integrais de análise não-linear via Método dos Elementos de Contorno, bem como as técnicas de integração de domínio.

Posteriormente serão discutidas, com a utilização do campo das tensões iniciais, as equações algébricas de análise não-linear via Método dos Elementos de Contorno. Em seguida, será dada uma breve explanação sobre os modelos não lineares escolhidos, que são o modelo escalar de dano e o modelo de dano para o concreto, vistos com um breve desenvolvimento.

Por último, será visto o desenvolvimento das equações não-lineares de dano escalar e do concreto, bem como o desenvolvimento das equações de dano em domínio estreito, que se degenerará em uma fratura não-linear.

\subsection{Campo das tensões iniciais}

A presença de campos iniciais de tensões ou deformações no domínio de um sólido, é de suma importância para problemas onde tais variáveis se relacionam com não-linearidades dos materiais, tais como plasticidade, dano e efeitos viscosos. Os campos de deformação ou tensão inicial são, por sua própria natureza, ideais para a utilização de métodos iterativo-incrementais, para a resolução de problemas nãolineares. As componentes de deformação em um ponto $s$ do domínio são dadas 
por:

$$
\epsilon_{i j}(s)=\epsilon_{i j}^{e}(s)+\epsilon_{i j}^{0}(s)
$$

onde $\epsilon_{i j}^{e}(s)$, representa o componente do tensor de deformações elásticas e $\epsilon_{i j}^{0}(s)$, o componente do tensor de deformações iniciais. Da mesma forma, pode-se escrever para o campo das tensões:

$$
\sigma_{i j}(s)=\sigma_{i j}^{e}(s)-\sigma_{i j}^{0}(s)
$$

A figura (5.1) mostra as relações das equações (5.1) e (5.2).

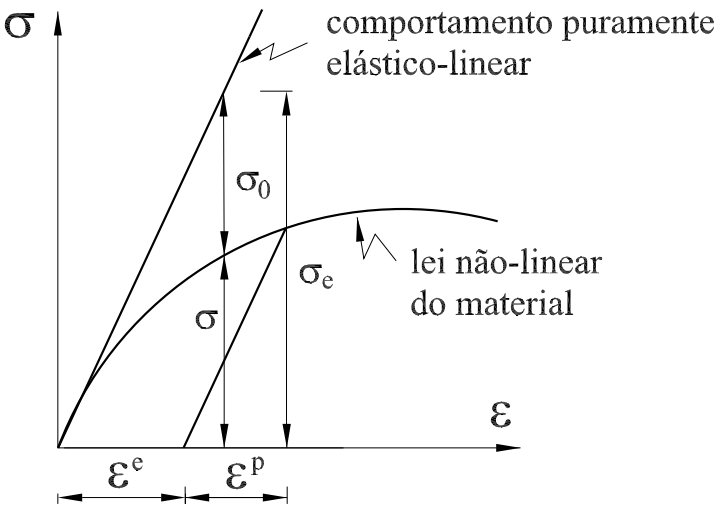

Fig. 5.1: Campo das tensões iniciais

As equações de equilíbrio sem forças volúmicas, podem ser escritas como:

$$
\frac{\partial \sigma_{i j}}{\partial x_{j}}=0 \quad \text { ou } \quad \frac{\partial \sigma_{i j}^{e}}{\partial x_{j}}-\frac{\partial \sigma_{i j}^{0}}{\partial x_{j}}=0
$$


O tensor $-\left(\partial \sigma^{0} / \partial x_{j}\right)$ é equivalente às forças de volume $\psi_{i}$. Introduzindo a força de volume $\psi_{i}$ na equação (5.3) tem-se:

$$
\frac{\partial \sigma_{i j}^{e}}{\partial x_{j}}-\frac{\partial \sigma_{i j}^{0}}{\partial x_{j}}+\psi_{i}=0
$$

Sobre o contorno, o vetor de deslocamentos $u_{i}$, é o mesmo para o caso elástico e para o caso das tensões reais que estão atuando no sólido, segundo modelo constitutivo. Porém, para as forças de superfície têm-se:

$$
p_{i}=\sigma_{i j} n_{j}=\left(\sigma_{i j}^{e}-\sigma_{i j}^{0}\right) n_{j}=p_{i}^{e}-p_{i}^{0}
$$

onde $p_{i}$, é a força de superfície 'verdadeira' que está atuando no contorno da estrutura, segundo alguma lei constitutiva não-linear, $p_{i}^{e}$ é a força de superfície totalmente elástica, $p_{i}^{0}$ a força de superfície inicial no contorno e $n_{j}$ é o vetor normal ao contorno.

Usando o teorema da divergência, o termo de integral de volume de (3.43) pode ser escrito como:

$$
\int_{\Omega} \psi_{i}(x) G_{i j}^{*}(x, \xi) d \Omega=\int_{\Omega} B_{i k j}^{*}(x, \xi) \sigma_{i k}^{0}(x) d \Omega-\int_{\Gamma} G_{i j}^{*}(x, \xi) p_{i}^{0}(x) d \Gamma
$$

onde $B_{i k j}^{*}(x, \xi)=\partial G_{i j}^{*}(x, \xi) / \partial x_{k}$.

Substituindo-se na equação (3.43) o termo de domínio de (5.6), lembrando que nesta equação $p_{i}=p_{i}^{e}$, e utilizando a relação da equação (5.5) para eliminar o termo $p_{i}^{0}$, obtém-se:

$$
c_{i j} u_{i}(\xi)=\int_{\Gamma}\left[G_{i j}^{*}(x, \xi) p_{j}(x)-H_{i j}^{*}(x, \xi) u_{j}(x)\right] d \Gamma+\int_{\Omega} B_{i k j}^{*}(x, \xi) \sigma_{i k}^{0}(x) d \Omega(5.7)
$$


O núcleo $B_{i k j}^{*}$ da integral de volume da equação (5.7), pode ser escrito como:

$$
B_{i k j}^{*}(x, \xi)=\frac{-1}{8 \pi(1-\nu) G r}\left[(1-2 \nu)\left(r_{, j} \delta_{i k}+r_{, k} \delta_{i j}\right)-r_{, i} \delta_{k j}+2 r_{, i} r_{, j} r_{, k}\right]
$$

Derivando-se a equação (5.8) e aplicando-se a relação constitutiva elástica, obtém-se:

$$
\begin{array}{r}
\sigma_{j k}(\xi)=\int_{\Gamma}\left[S_{i j k}^{*}(x, \xi) p_{i}(x)-D_{i j k}^{*}(x, \xi) u_{i}(x)\right] d \Gamma+ \\
\int_{\Omega} E_{i p j k}^{*}(x, \xi) \sigma_{i p}^{0}(x) d \Omega-\frac{1}{8(1-\nu)}\left[2 \sigma_{j k}^{0}(s)+(1-4 \nu) \sigma_{m m}^{0} \delta_{j k}\right]
\end{array}
$$

sendo:

$$
\begin{array}{r}
E_{i p j k}^{*}(x, \xi)=\frac{1}{4 \pi(1-\nu) r^{2}}\left[(1-2 \nu)\left(\delta_{i j} \delta_{k p}+\delta_{i k} \delta_{j p}\right)-\delta_{i p} \delta_{j k}+2 \nu\right. \\
\left(\delta_{i j} r_{, k} r_{, p}+\delta_{i k} r_{, j} r_{p}+\delta_{j p} r_{, i} r_{, k}+\delta_{k p} r_{, i} r_{, j}\right)+2\left(\delta_{j k} r_{, i} r_{, p}+\right. \\
\left.\left.+\delta_{i p} r_{, j} r_{, k}\right)-8 r_{, i} r_{, p} r_{, j} r_{, k}\right]
\end{array}
$$

O último termo da equação (5.9) é o conhecido termo livre. Ele surge da derivação do núcleo da integral com o termo de tensão inicial da identidade Somagliana. Neste trabalho, para evitar se escrever as equações de tensões para o contorno, pois há fortes singularidades com estas integrais nesta região, optou-se pela utilização de nós duplos nos vértices das células que pertencem ao contorno, onde tais nós são deslocados para o domínio. 


\subsubsection{Discretização das Integrais de Domínio - Elementos com aproximação linear}

A consideração de campos iniciais de tensão para tratar os problemas nãolineares, introduz as integrais de domínio nas equações do método dos elementos de contorno para problemas de elasticidade plana. Neste trabalho, o domínio foi discretizado em células triangulares, onde as deformações e tensões serão aproximados para os vértices das células por funções aproximadoras com variações lineares, e depois somadas as contribuições de cada célula as quais o nó pertence.

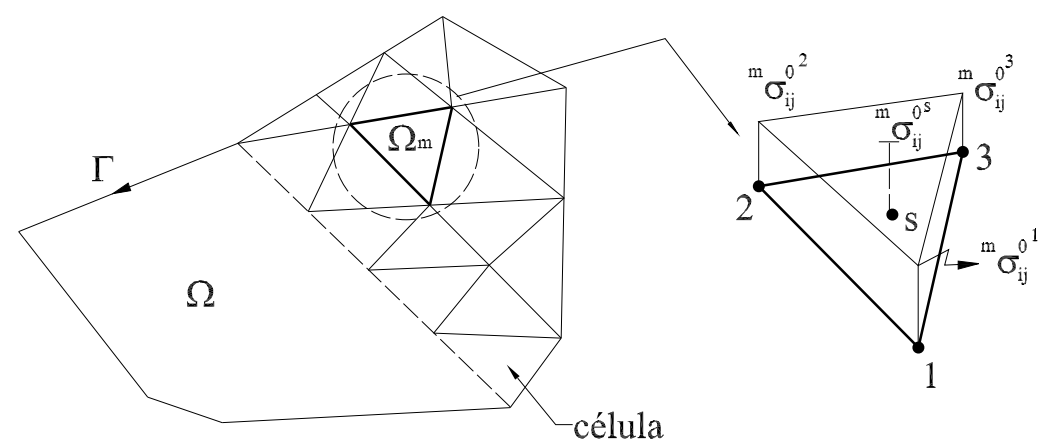

Fig. 5.2: Discretização do domínio em células triangulares

As variáveis de domínio neste caso são as tensões iniciais. O domínio $\Omega$ é discretizado em $N_{c}$ células onde $\Omega=\bigcup_{m+1}^{N_{c}} \Omega_{m}$. As funções aproximadoras de deformações são da forma polinomial e contribuem com cada nó na seguinte forma.

$$
{ }^{m} \sigma_{i j}^{0}=\phi_{k}(\xi)^{m} \sigma_{i j}^{0^{m}}
$$

onde $m$, representa cada célula $\Omega_{m}$ e o índice $k$, os nós da célula. ${ }^{m} \sigma_{0^{k}}^{m}$ representa a variável nodal da componente $i j$ do tensor de tensões iniciais da célula $m$ para o nó $k$. O termo de domínio da equação (5.7), pode ser escrito como uma contribuição das integrais de cada célula aproximada: 


$$
\int_{\Omega} B_{i k j}^{*}(x, \xi) \sigma_{k j}^{0} d \Omega=\sum_{1}^{N_{c}} \int_{\Omega_{m}} B_{i k j}^{*}(x, \xi)^{m} \phi_{l}(\xi) \sigma_{k j}^{0} d \Omega_{m}
$$

A função aproximadora é escrita em forma de coordenadas cilíndricas (Botta, 2003).

$$
\phi_{i}(r, \theta)=\frac{a_{i}^{0}}{2 A}+\omega_{i}[x(s)+r \cdot \cos (\theta)]+\eta_{i}[y(s)+r \cdot \operatorname{sen}(\theta)]
$$

onde $A$ é a área do elemento triangular, cujos vértices coincidem com os nós de tensão, $x(x)$ e $y(s)$ são as coordenadas cartesianas do ponto fonte $s$ em relação a um sistema $(x, y)$ qualquer adotado. Os outros coeficientes da equação (5.13) são dados por:

$$
a_{i}^{0}=x_{j} y_{k}-y_{j} x_{k} \quad \omega_{i}=y_{j}-y_{k} \quad \eta_{i}=x_{k}-x_{j}
$$

para $i, j$ e $k$ variando ciclicamente de 1 a 3 . O índice subscrito na equação (5.14) representa os nós nas células com coordenadas $\left(x_{m}, y_{m}\right)$.

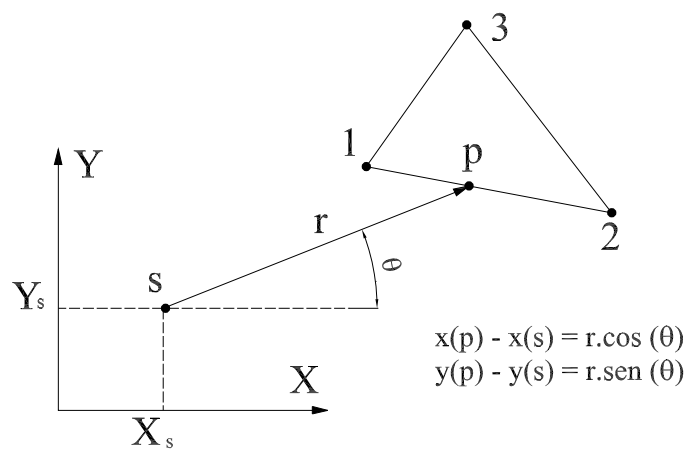

Fig. 5.3: Coordenadas cilíndricas $r$ e $\theta$ 
Na figura (5.3), são mostrados o raio e o ângulo $\theta$ da equação (5.13), bem como todas as variáveis da função de forma.

A técnica utilizada, consistiu em se fazer primeiramente, uma integração analítica na equação (5.12) em relação à $r$. Em seguida, substitui-se o valor de $r$ de acordo com a figura (5.4) e da relação (5.17), onde 'a' é a menor distância entre o ponto fonte e a aresta da célula que está sendo integrada.

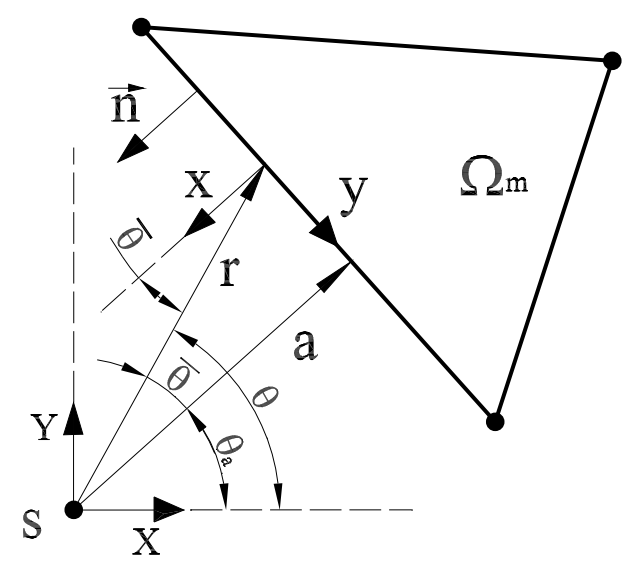

Fig. 5.4: Relação entre o eixo global e o eixo local da aresta da célula

Posteriormente, integra-se também analiticamente em relação a $\theta$ e, diferentemente do caso das integrais de contorno, as integrais precisam ser resolvidas em coordenadas globais, com isso tem-se, como mostra a figura (5.4), a relação entre o ângulo $\bar{\theta}$, que é o ângulo entre o raio $r$ e a reta $a$, e o ângulo $\theta$ global dado por:

$$
\begin{array}{r}
\theta=\bar{\theta}+\theta_{a} \\
d \theta=d \bar{\theta}
\end{array}
$$

O raio de integração entre o ponto fonte e o elemento pode ser escrito por:

$$
r=\frac{a}{\cos (\bar{\theta})}
$$




$$
d r=\frac{a * \operatorname{sen}(\bar{\theta})}{\cos (\bar{\theta})^{2}} d \bar{\theta}
$$

e o elemento infinitesimal de área $d \Omega$, da equação (5.9) a ser integrada em cada célula, pode ser substituído pela relação:

$$
d \Omega=r d r d \theta
$$

As relações determinadas pelas equações $(5.15),(5.16),(5.17),(5.18)$ e (5.19), são substituídas nas equações integrais de domínio de deformação (5.8). Faz-se uma integração analítica, primeiramente em relação ao raio $r$ e, posteriormente, substituem-se os termos do raio com a relação (5.17), integrando-se analiticamente em relação a variável $\bar{\theta}$.

Com relação às equações de tensão, têm-se problemas de singularidade quando o ponto fonte pertencer ao contorno. Para evitar tal problema, os nós das células pertencentes ao contorno são afastados para dentro da célula, como mostra a figura $(5.5)$.

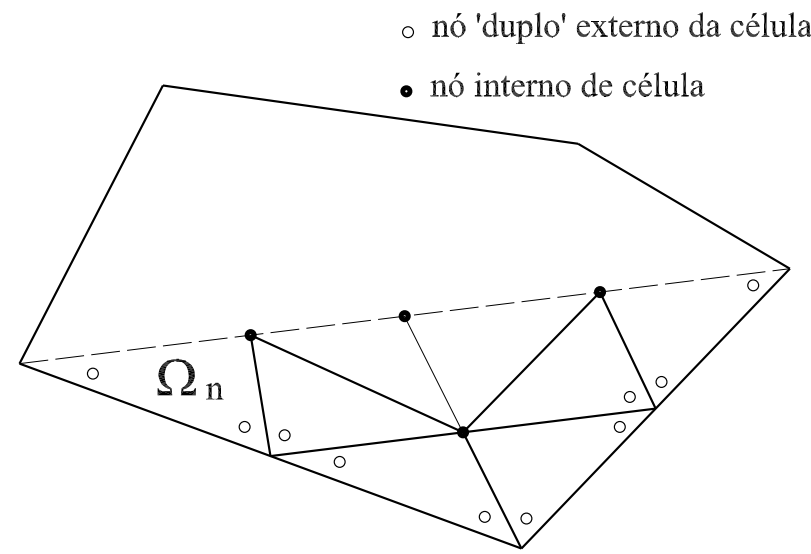

Fig. 5.5: Nós internos e externos ao contorno da célula

Outro problema referente à equação de tensão no domínio, equação (5.10), é sua impossibilidade de ser integrada analiticamente. Para contornar este problema, 
foi adotada a integração em elemento de linha na célula, como será demonstrado a seguir.

\subsubsection{Integração em linha das integrais de domínio}

A solução fundamental $E_{i p j k}$ da equação (5.10) possui restrições para integração analítica. Utilizando-se a relação de compatibilidade de deslocamentos, pode-se escrever o último termo da equação (5.7) por:

$$
c_{i j} u_{i}(\xi)=\cdots+\int_{\Omega} \frac{\partial G_{i j}^{*}}{\partial x_{k}}(x, \xi) \sigma_{i k}^{0}(x) d \Omega
$$

integrando por partes o último termo de (5.7), já substituído pelo termo de (5.19), tem-se:

$$
c_{i j} u_{i}(\xi)=\cdots+\int_{\Gamma} G_{i j}^{*}(x, \xi) \sigma_{j k}^{0} n_{k}(x) d \Gamma-\int_{\Omega} G_{i j}^{*}(x, \xi) \sigma_{j k, k}^{0}(x) d \Omega
$$

onde $n$ é a normal do elemento a ser integrado. O termo $\sigma_{j k, k}^{0}$, integrado sobre o domínio, pode ser entendido como um carregamento de domínio, podendo então ser escrito apenas derivando a função aproximadora:

$$
\sigma_{l k, k}=\frac{\partial \phi_{m}}{\partial x_{k}} \sigma_{l k}
$$

onde o termo $\phi$ é a função aproximadora para um elemento $m$ qualquer.

Aplicando-se a lei de Hooke sobre a equação (5.20) e, utilizando-se a expressão (5.21), têm-se os termos de tensão:

$$
\sigma_{i j}(\xi)=\cdots+\int_{\Gamma} D_{i j k}^{*}(x, \xi) \sigma_{j k}^{0} n_{k}(x) d \Gamma-\int_{\Omega} D_{i j k}^{*}(x, \xi) \frac{\partial \phi_{m}}{\partial x_{k}} \sigma_{j k}^{0}(x) d \Omega
$$


O primeiro termo da equação (5.22) será integrado no contorno, como na equação (3.52). Através da relação $d \Omega=r d r d \theta$ e derivando-se a equação (5.13), pode-se integrar o segundo termo de (5.22), primeiramente em relação a $r$ e, posteriormente, em relação a $\theta$, sendo essas duas integrais feitas analiticamente. Matricialmente, o termo $E_{i j k l}$ pode agora ser escrito para um nó $k$ de uma célula $m$ qualquer, como:

$$
\begin{gathered}
\boldsymbol{E}^{*}=\left\{\left[\begin{array}{cc}
D_{111}^{*} & D_{112}^{*} \\
D_{121}^{*} & D_{122}^{*} \\
D_{221}^{*} & D_{222}^{*}
\end{array}\right]\left[\begin{array}{ccc}
n_{1} & n_{2} & 0 \\
0 & n_{1} & n_{2}
\end{array}\right]+\right. \\
\left.-\left[\begin{array}{cc}
D_{111}^{\prime *} & D_{112}^{\prime *} \\
D_{121}^{\prime *} & D_{122}^{\prime *} \\
D_{221}^{\prime *} & D_{222}^{\prime *}
\end{array}\right]\left[\begin{array}{ccc}
\phi_{m, x} & \phi_{m, y} & 0 \\
0 & \phi_{m, x} & \phi_{m, y}
\end{array}\right]\right\}\left\{\begin{array}{c}
\sigma_{11_{m}}^{k} \\
\sigma_{12_{m}}^{k} \\
\sigma_{22_{m}}^{k}
\end{array}\right\}
\end{gathered}
$$

sendo o termo $D^{\prime *}$ da solução fundamental integrada no domínio da célula, como mostra a equação (5.22). O termo livre da expressão (5.9), é suprimido pelo desenvolvimento da expressão (5.22), sendo que o termo livre agora tem valor unitário no ponto fonte apenas.

\subsubsection{Discretização das Integrais de Domínio - Elementos com aproximação constante}

Para o caso de análise de uma sub-região muito 'fina', que se degenerará segundo leis não lineares da mecânica do dano dando origem à fratura, optou-se, por uma aproximação constante das células internas da sub-região. Como mostra a figura (5.6).

Neste caso, a função aproximadora da equação (5.13), terá valor unitário e pode ser escrita por:

$$
\phi_{i}(\text { const })=1
$$




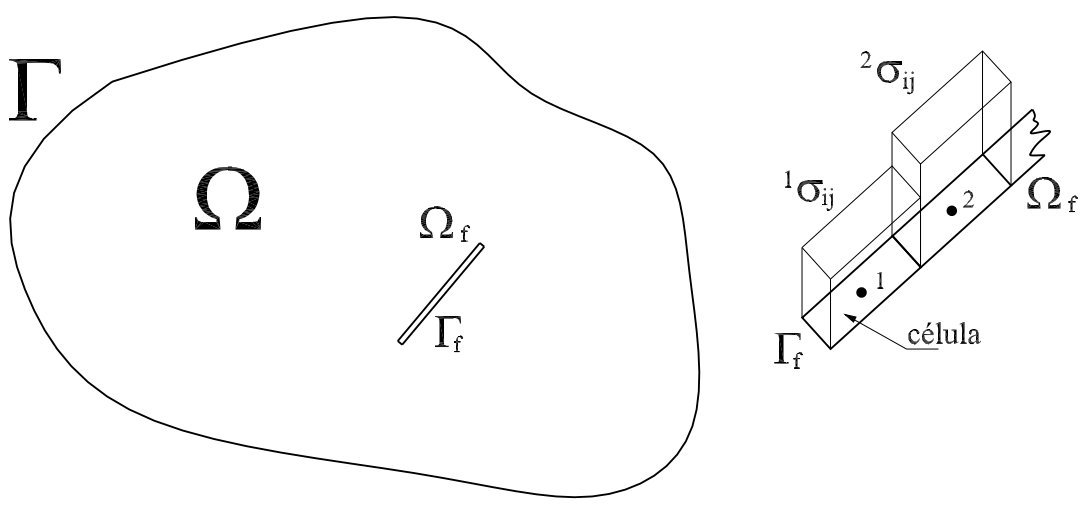

Fig. 5.6: Elemento de domínio com aproximação constante

Com isto, somente o termo $B_{i j k}^{*}$ será integrado normalmente na célula, enquanto que, o termo da solução fundamental de tensão dada pela equação (5.23), será escrito como:

$$
\boldsymbol{E}^{*}=\left[\begin{array}{cc}
D_{111}^{*} & D_{112}^{*} \\
D_{121}^{*} & D_{122}^{*} \\
D_{221}^{*} & D_{222}^{*}
\end{array}\right]\left[\begin{array}{ccc}
n_{1} & n_{2} & 0 \\
0 & n_{1} & n_{2}
\end{array}\right]\left\{\begin{array}{c}
\sigma_{11_{m}}^{k} \\
\sigma_{12_{m}}^{k} \\
\sigma_{22_{m}}^{k}
\end{array}\right\}
$$

pois como a função aproximadora é uma constante, o termo derivado $\phi_{m, k}$ será nulo.

\subsection{Análise não-linear via Método dos Elementos de Contorno}

$\mathrm{Na}$ análise de problemas físicos não-lineares, utilizando-se o método dos elementos de contorno, pode-se utilizar a introdução de tensões iniciais aplicadas no domínio. A relação constitutiva do modelo não linear está ligada à definição da tensão inicial, sendo esta definida como a diferença entre a tensão que segue a lei elástica e a tensão dita como verdadeira, que nada mais é do que a tensão real segundo a lei constitutiva não linear do material. 
Escrevendo as equações matriciais de deslocamentos (3.56) e tensões (3.60), introduzindo o campo das tensões iniciais, tem-se:

$$
\begin{array}{r}
{[\boldsymbol{H}]\{\boldsymbol{U}\}=[\boldsymbol{G}]\{\boldsymbol{P}\}+[\boldsymbol{Q}]\left\{\boldsymbol{\sigma}_{\mathbf{0}}\right\}} \\
\{\boldsymbol{\sigma}\}=-[\boldsymbol{S}]\{\boldsymbol{U}\}+[\boldsymbol{D}]\{\boldsymbol{P}\}+\left[\boldsymbol{Q}^{\prime}\right]\left\{\boldsymbol{\sigma}_{\mathbf{0}}\right\}
\end{array}
$$

onde $\boldsymbol{Q}$ e $\boldsymbol{Q}^{\prime}$ são as matrizes de influência das soluções fundamentais (5.8) e (5.23) Separando as forças e deslocamentos prescritos, tem-se, a partir da equação $(5.26)$ :

$$
[\boldsymbol{A}]\{\boldsymbol{X}\}=[\boldsymbol{B}]\{\boldsymbol{Y}\}+[\boldsymbol{Q}]\left\{\boldsymbol{\sigma}_{0}\right\}
$$

onde na matriz $[\boldsymbol{A}]$ tem-se os termos da matriz $[\boldsymbol{H}]$ e $[\boldsymbol{G}]$, que multiplicam termos incógnitos de deslocamentos e forças de superfície, respectivamente, enquanto a matriz $[\boldsymbol{B}]$ tem influência sobre os valores prescritos de contorno, os quais são representados pelo vetor $\{\boldsymbol{Y}\}$. Multiplicando-se os termos prescritos de (5.28),tem-se:

$$
[\boldsymbol{A}]\{\boldsymbol{X}\}=\{\boldsymbol{F}\}+[\boldsymbol{Q}]\left\{\boldsymbol{\sigma}_{\mathbf{0}}\right\}
$$

Separando-se, também na equação (5.27), os termos incógnitos e prescritos do contorno, juntamente com os respectivos termos das matrizes de influência, tem-se:

$$
\{\boldsymbol{\sigma}\}=-\left[\boldsymbol{A}^{\prime}\right]\{\boldsymbol{X}\}+\left\{\boldsymbol{F}^{\prime}\right\}+\left[\boldsymbol{Q}^{\prime}\right]\left\{\boldsymbol{\sigma}_{\mathbf{0}}\right\}
$$

onde $\left[\boldsymbol{A}^{\prime}\right]$ e $\left\{\boldsymbol{F}^{\prime}\right\}$ tem o mesmo significado de $[\boldsymbol{A}]$ e $\{\boldsymbol{F}\}$, respectivamente. Isolandose o termo incógnito da equação (5.29), tem-se: 


$$
\{\boldsymbol{X}\}=[\boldsymbol{A}]^{-1}\left\{\{\boldsymbol{F}\}+[\boldsymbol{Q}]\left\{\boldsymbol{\sigma}_{\mathbf{0}}\right\}\right\}
$$

A equação (5.31), pode ser escrita como:

$$
\{\boldsymbol{X}\}=\{\boldsymbol{M}\}+[\boldsymbol{R}]\left\{\boldsymbol{\sigma}_{\mathbf{0}}\right\}
$$

onde:

$$
\begin{array}{r}
\{\boldsymbol{M}\}=[\boldsymbol{A}]^{-1}\{\boldsymbol{F}\} \\
{[\boldsymbol{R}]=[\boldsymbol{A}]^{-1}[\boldsymbol{Q}]}
\end{array}
$$

Substituindo-se (5.32) em (5.30), tem-se:

$$
\{\boldsymbol{\sigma}\}=\{\boldsymbol{N}\}+[\boldsymbol{S}]\left\{\boldsymbol{\sigma}_{\mathbf{0}}\right\}
$$

sendo que os termos $\boldsymbol{N}$ e $\boldsymbol{S}$, descritos na equação (5.34), são representados por:

$$
\begin{array}{r}
\{\boldsymbol{N}\}=-\left[\boldsymbol{A}^{\prime}\right][\boldsymbol{M}]+\left\{\boldsymbol{F}^{\prime}\right\}=-\left[\boldsymbol{A}^{\prime}\right][\boldsymbol{A}]^{-1}\{\boldsymbol{F}\}+\left\{\boldsymbol{F}^{\prime}\right\} \\
{[\boldsymbol{S}]=-\left[\boldsymbol{A}^{\prime}\right][\boldsymbol{R}]+\left[\boldsymbol{Q}^{\prime}\right]=-\left[\boldsymbol{A}^{\prime}\right][\boldsymbol{A}]^{-1}[\boldsymbol{Q}]+\left[\boldsymbol{Q}^{\prime}\right]}
\end{array}
$$

Adicionando o campo de tensão inicial nos dois lados da equação (5.34) tem-se: 


$$
\left\{\boldsymbol{\sigma}^{e}\right\}=\{\boldsymbol{N}\}+[\overline{\boldsymbol{S}}]\left\{\boldsymbol{\sigma}_{\mathbf{0}}\right\}
$$

sendo que $[\overline{\boldsymbol{S}}]=[\boldsymbol{S}]+[\boldsymbol{I}]$, onde $[\boldsymbol{I}]$ é a matriz identidade. O termo $\left\{\boldsymbol{\sigma}^{e}\right\}=$ $\{\boldsymbol{\sigma}\}+\left\{\boldsymbol{\sigma}_{\mathbf{0}}\right\}$, segundo figura (5.1), é a tensão elástica, isto é, é igual à soma da tensão que realmente atua na estrutura segundo uma lei constitutiva, mais o acréscimo de uma tensão inicial, a qual pode ser introduzida inicialmente como parte de um processo iterativo incremental utilizado para se resolverem problemas não lineares. A equação (5.37), pode ser escrita em termos de deformações, segundo a lei constitutiva linear por:

$$
\{\boldsymbol{Y}(\boldsymbol{\epsilon})\}=[\boldsymbol{E}]\{\boldsymbol{\epsilon}\}+\{\boldsymbol{N}\}+[\overline{\boldsymbol{S}}]\{[\boldsymbol{E}]\{\boldsymbol{\epsilon}\}-\{\boldsymbol{\sigma}(\boldsymbol{\epsilon})\}\}=\mathbf{0}
$$

onde $[\boldsymbol{E}]$ é a matriz com os módulos elásticos de rigidez, e $\{\boldsymbol{\sigma}(\boldsymbol{\epsilon})\}$ é o vetor com as tensões decorrentes do modelo constitutivo não-linear do material. A equação de equilíbrio não-linear $\{\boldsymbol{Y}(\boldsymbol{\epsilon})\}=\mathbf{0}$, é geral para qualquer lei constitutiva não linear, tornando tal formulação, única e aplicável a uma vasta gama de modelos não lineares. No ítem seguinte, será apresentado o modelo não linear de dano escalar, no qual será discutido o procedimento incremental-iterativo para a resolução do sistema não-linear de (5.38).

\subsection{Mecânica do Dano}

Um dos comportamentos não-lineares que as estruturas granulares, como o concreto, apresentam de mais significativo, se refere ao processo de danificação. Esse processo consiste de microdefeitos constituídos por inclusões ou mesmo vazios dentro de um sólido, os quais, por suas características, favorecem o aparecimento de concentração de tensões. 
O dano pode progredir, seja por solicitação mecânica ou por condições ambientais, dando origem a rompimentos entre partículas, que afetam as características mecânicas do sólido analisado segundo a Mecânica do Contínuo.

A mecânica do dano consiste em se analisar o aparecimento e a progressão desses microdefeitos, que estão difusos em regiões de um sólido contínuo, onde as características elásticas do sólido serão penalizadas de acordo com a evolução deste dano.

Com o incremento da propagação do dano, o mesmo vai se intensificando em uma região cada vez mais estreita, podendo dar origem a uma fratura discreta.

A figura (5.7) mostra a relação tensão $\times$ deformação para o caso de dano escalar. Nota-se que o material possui um comportamento elástico até o ponto $\sigma_{e}$ com $D=0$ e depois vai perdendo sua rigidez até possuir tensão nula $D=1$. A tensão máxima é $\sigma_{m}$.

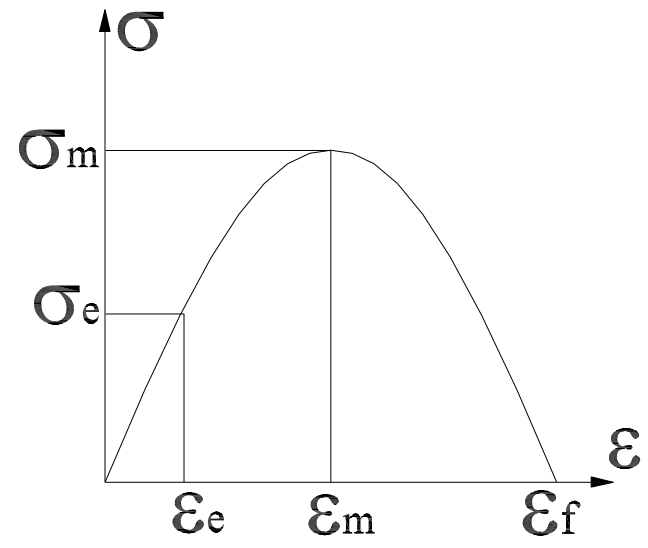

Fig. 5.7: Relação tensão x deformação - Modelo de Dano

\subsubsection{Dano Simples}

O modelo de dano isotrópico, isto é, que se propaga igualmente em todas as direções, é definido pelo conjunto de equações.

$$
\Psi=\frac{1}{2} E(1-D) \epsilon^{2}
$$




$$
\begin{array}{r}
Y=-\frac{\partial Y}{\partial D}=\frac{1}{2} E \epsilon^{2} \\
\sigma=\frac{\partial Y}{\partial \epsilon}=(1-D) E \epsilon \\
\dot{D}=\dot{\gamma} \frac{\partial f(Y, D)}{\partial Y} \\
f(Y, D)=Y-Y_{0}-M D \\
f \leq 0, \quad f \geq 0, \quad f \dot{\gamma}=0
\end{array}
$$

onde $\Psi$ é a energia livre de Helmoltz, $D$ é a única variável escalar de dano, que tem o valor de 0 no início, isto é, o material está íntegro no início indo até o valor de 1 quando o material está totalmente deteriorado, $Y$ é a tensão termodinâmica conjugada à $D$. O termo $f$ é a função de carregamento e, juntamente com o multiplicador $\dot{\gamma}$, satisfazem as condições de Kuhn-Tucker, expressa em (5.44). $M$ e $Y_{0}$ são parâmetros constantes do material.

Todas as variáveis são escalares por ser o problema unidimensional; pela condição de consistência $\dot{f}=0$, podem ser escritas as relações:

$$
\begin{array}{r}
\dot{f}(Y, D)=\frac{\partial f}{\partial Y} \dot{Y}+\frac{\partial f}{\partial D} \dot{D}=0 \\
\dot{D}=\frac{\dot{Y}}{M}=\frac{E \epsilon}{M} \dot{\epsilon}
\end{array}
$$

A relação constitutiva é dada por:

$$
\left\{\begin{array}{cc}
\dot{\sigma}=(1-D) E \dot{\epsilon} & \text { na parte elástica } \\
\dot{\sigma}=(1-D) E \dot{\epsilon}-\dot{D} E \epsilon=\left[(1-D) E-\frac{(E \epsilon)^{2}}{M}\right] & \text { na parte com } \dot{D}>0
\end{array}\right.
$$

Para o caso bidimensional, têm-se as relações constitutivas, com os tensores de tensão derivando da energia livre de Helmoltz. 


$$
\rho \Psi=\rho \Psi\left(\epsilon_{i j}, D\right)=\frac{1}{2}(1-D) E_{i j k l} \epsilon_{i j} \epsilon_{k l}
$$

onde $\rho$ é a densidade de massa, $E_{i j k l}$ as componentes $i j k l$ do tensor constitutivo elástico de quarta ordem do material íntegro e $\epsilon_{i j}$ as componentes $i j$ do tensor de deformações.

O tensor de tensões é dado pelas componentes:

$$
\sigma_{i j}=\rho \frac{\partial \Psi}{\partial \epsilon_{i j}}=\frac{1}{2}(1-D) E_{i j k l} \epsilon_{k l}
$$

e a força termodinâmica $Y$ associada à $D$ é dada por:

$$
Y=-\rho \frac{\partial \Psi}{\partial D}=\frac{1}{2} E_{i j k l} \epsilon_{i j}^{e} \epsilon_{k l}^{e}
$$

A função de carregamento $f(Y, D)$ é definida por:

$$
f(Y, D)=Y-k(D)
$$

onde $k(D)$ mede o maior valor atingido até então por $Y$. Neste modelo, $k(D)$ é linear com $D$, tomando a forma de :

$$
k(D)=Y_{0}+M D
$$

sendo $Y_{0}$ e $M$ constantes do material. 
O início do processo de danificação e sua propagação ocorrem de acordo com o critério de danificação:

$$
\dot{D}=\dot{\lambda} \frac{\partial f(Y, D)}{\partial Y}
$$

o multiplicador $\dot{\lambda}$ deve satisfazer as condições de Kuhn-Tucker:

$$
\dot{\lambda} \geq 0, \quad f \leq 0, \quad \dot{\lambda} f=0
$$

O multiplicador $\dot{\lambda}$ é calculado da condição de consistência e, quando positivo, é dado por:

$$
\dot{f}(Y, D)=\frac{\partial f}{\partial Y} \dot{Y}+\frac{\partial f}{\partial D} \dot{D}
$$

Com as relações $(5.55),(5.51)$ e (5.52) tem-se:

$$
\dot{\lambda}=\dot{D}=\left(\frac{\dot{Y}}{M}\right)
$$

\subsubsection{Processo não-linear incremental - Dano Simples}

Como em todo processo de resolução de problemas não-lineares, aplicar-se-á o procedimento de passo finito para linearizar o problema em questão. A solução passa pela discretização do problema no campo temporal e espacial. Na primeira, resulta na discretização de intervalos de tempo $\Delta_{t}=t_{n+1}-t_{n}$, ou passos de carga, sendo $t_{n}$ e $t_{n+1}$ dois instantes consecutivos de tempo. No intervalo $\Delta_{t}$, o problema é 
não linear e deve ser resolvido por tentativas lineares, sendo que, o resultado dever ser corrigido pelas leis constitutivas não lineares do material.

O problema, em certo instante de tempo $\Delta_{t}$, consiste em manter o equilíbrio estático do sólido, dadas a várias condições de contorno impostas, tais como deslocamentos, forças de superfície e de volume, garantindo também a validade das equações de compatibilidade, como mostram as equações:

$$
\begin{array}{rrr}
\operatorname{div} \sigma_{i+1}+b_{n+1}=0 & \text { em } & \Omega \\
\frac{1}{2}\left[\operatorname{grad} u_{n+1}+\operatorname{grad}^{T} u_{n+1}\right]=\epsilon_{n+1}=0 & \text { em } & \Omega \\
m \cdot \sigma_{n+1}=p_{n+1} & \text { em } & \Gamma_{b} \\
u_{n+1}=U_{n+1} & \text { em } & \Gamma_{a}
\end{array}
$$

onde tem-se os incrementos para cada passo de carga de $\Delta b_{n}=b_{n+1}-b_{n}, \Delta p_{n}=$ $p_{n+1}-p_{n}$ e $\Delta U_{n}=U_{n+1}-U_{n}$ para os valores de deslocamentos e forças de superfície prescritos nos contornos $\Gamma_{a}$ e $\Gamma_{b}$, respectivamente. Sendo $\sigma_{n+1}$ e $u_{n+1}$, os tensores de tensão e de deslocamentos dos pontos de domínio do sólido. Às condições de equilíbrio e de compatibilidade (5.57)-(5.60), acrescentam-se a equação da lei constitutiva não linear de dano local, juntamente com as condições de Kuhn-Tucker.

A discretização espacial é feita via aproximação das variáveis de contorno e de domínio, resultando no sistema algébrico do método dos elementos de contorno. Utilizando-se a equação (5.38), agora escrita em passo de carga, para o incremento $n$, tem-se:

$$
\left\{Y\left(\Delta \epsilon_{n}\right)\right\}=[E]\left\{\Delta \epsilon_{n}\right\}+\left\{\Delta N_{n}\right\}+[\bar{S}]\left\{[E]\left\{\Delta \epsilon_{n}\right\}-\left\{\Delta \sigma_{n}\left(\Delta \epsilon_{n}\right)\right\}\right\}=0(5.61)
$$

a equação (5.61) respeita as condições de (5.57)-(5.60) e está escrita em função de variáveis incrementais $\boldsymbol{\Delta} \boldsymbol{\epsilon}_{\boldsymbol{n}}$. Utilizando-se um processo iterativo, tipo NewtonRaphson com fases de previsão e correção, tem-se para cada iteração: 


$$
\left\{\Delta \epsilon_{n}^{i+1}\right\}=\left\{\Delta \epsilon_{n}^{i}\right\}+\left\{\delta \Delta \epsilon_{n}^{i+1}\right\}
$$

onde $i$ indica cada iteração. Determina-se o valor de $\left\{\delta \Delta \epsilon_{n}^{i+1}\right\}$ para cada iteração e truncando-se a série de Taylor no primeiro termo da expressão de $Y\left(\left\{\Delta \epsilon_{n}^{i}\right\}\right)$ em (5.61), tem-se:

$$
Y\left(\left\{\Delta \epsilon_{n}^{i}\right\}\right)=\frac{\partial\left\{Y\left(\left\{\Delta \epsilon_{n}^{i}\right\}\right)\right\}}{\partial\left\{\Delta \epsilon_{n}^{i}\right\}} \cdot\left\{\delta \Delta \epsilon_{n}^{i}\right\}+\cdots=0
$$

utilizando-se o primeiro termo da equação (5.63) e derivando-se a equação (5.61) em relação a $\Delta \epsilon_{n}^{i}$, tem-se:

$$
\left\{Y\left(\left\{\Delta \epsilon_{n}^{i}\right\}\right)\right\}=\left\{[E]-[\bar{S}]\left([E]-\left[\frac{\partial \Delta \sigma_{n}^{i}}{\partial \Delta \epsilon_{n}^{i}}\right]\right)\right\} \cdot\left\{\delta \Delta \epsilon_{n}^{i}\right\}
$$

onde o termo que multiplica $\left\{\delta \Delta \epsilon_{n}^{i}\right\}$ é conhecido como operador tangente consistente algorítmico, diferindo do operador tangente contínuo pela variável em relação à qual se calcula a derivada da equação de equilíbrio.

Sendo as equações resolvida por incrementos, nada mais natural que se tomarem as derivadas em relação as variáveis incrementais, daí o operador ser consistente com o algorítimo do problema incremental. O operador consistente tende ao operador contínuo quando $\Delta \epsilon$ tende a zero, ou seja:

$$
\begin{array}{r}
C^{t}=\frac{\partial \sigma}{\partial \epsilon} \\
C^{t C}=\frac{\partial \Delta \sigma}{\partial \Delta \epsilon} \\
C^{t C}=C^{t} \text { para } \Delta \epsilon \rightarrow 0
\end{array}
$$


sendo $\boldsymbol{C}^{\boldsymbol{t}}$ e $\boldsymbol{C}^{\boldsymbol{t} \boldsymbol{C}}$ os operadores tangente contínuo e consistente, respectivamente.

Na expressão (5.64), surge a derivada dos incrementos de tensões em relação ao incremento de deformações e, para cada lei constitutiva não linear, tal operação deve ser explicitada. Considerando-se o modelo de dano local simples, tal relação constitutiva pode ser escrita como.

$$
\dot{\boldsymbol{\sigma}}=(1-D) \boldsymbol{E} \dot{\boldsymbol{\epsilon}}-\boldsymbol{E} \boldsymbol{\epsilon} \dot{D}
$$

onde $\boldsymbol{E}$ é o tensor constitutivo elástico de quarta ordem, $\dot{\boldsymbol{\sigma}}, \dot{\boldsymbol{\epsilon}}$ e $\dot{D}$ são as taxas dos tensores de tensões, de deformações e da variável de dano escalar, respectivamente.

Em variáveis incrementais, a expressão (5.66) pode ser escrita como:

$$
\boldsymbol{\Delta} \boldsymbol{\sigma}_{\boldsymbol{n}}=\left(1-D_{n}-\Delta D_{n}\right) \boldsymbol{E}: \Delta \boldsymbol{\epsilon}_{\boldsymbol{n}}-\Delta D_{n} \boldsymbol{E}: \boldsymbol{\epsilon}_{\boldsymbol{n}}
$$

a matriz tangente consistente é dada pela derivada.

$$
\left[\frac{\partial \Delta \sigma_{n}}{\partial \Delta \epsilon_{n}}\right]=\left(1-D_{n}-\Delta D_{n}\right)[E]-\left\{E:\left(\epsilon_{n}+\Delta \epsilon_{n}\right)\right\} \otimes\left\{\frac{\partial \Delta D_{n}}{\partial \Delta \epsilon_{n}}\right\}
$$

sendo $D_{n}$ e $\epsilon_{n}$ conhecidos no tempo $t_{n}$ e $\Delta D_{n}$ e $\Delta \epsilon_{n}$ as variáveis incrementais.

Da condição de consistência do modelo, $\dot{f} \dot{D}=0$, para que haja incremento do dano, ou seja, $\dot{D}>0$, deve-se obedecer a condição $\dot{f}=0$, sendo então:

$$
\begin{aligned}
f & =Y-Y_{0}-M D \\
\Delta f_{n} & =\Delta Y_{n}-M \Delta D_{n}
\end{aligned}
$$




$$
\begin{array}{r}
\dot{f}=\frac{\partial f}{\partial Y} \frac{\partial Y}{\partial \epsilon} \dot{\epsilon}+\frac{\partial f}{\partial D} \dot{D}=0 \\
\Delta f_{n}=\frac{\partial \Delta f_{n}}{\partial \Delta Y_{n}} \frac{\partial \Delta Y_{n}}{\partial \Delta \epsilon_{n}} \Delta \epsilon_{n}+\frac{\partial \Delta f_{n}}{\partial \Delta D_{n}} \Delta D_{n}=0
\end{array}
$$

utilizando-se a última expressão de (5.69), pode-se escrever:

$$
\Delta D_{n}=-\frac{\left[\frac{\partial \Delta f_{n}}{\partial \Delta Y_{n}} \frac{\partial \Delta Y_{n}}{\partial \Delta \epsilon_{n}}\right]}{\frac{\partial \Delta f_{n}}{\partial \Delta D_{n}}} \Delta \epsilon_{n}=\frac{1 \frac{\partial \Delta Y_{n}}{\partial \Delta \epsilon_{n}}}{M} \Delta \epsilon_{n}
$$

Segundo o modelo de dano simples, pode-se escrever as equações matriciais para esse modelo:

$$
\begin{array}{r}
Y=\frac{1}{2}\{\epsilon\}:[E]:\{\epsilon\} \\
\Delta Y_{n}=\left\{\epsilon_{n}\right\}:[E]:\left\{\Delta \epsilon_{n}\right\}+\frac{1}{2}\left\{\Delta \epsilon_{n}\right\}:[E]:\left\{\Delta \epsilon_{n}\right\} \\
\left\{\frac{\partial \Delta Y_{n}}{\partial \Delta \epsilon_{n}}\right\}=[E]:\left(\left\{\epsilon_{n}\right\}+\left\{\Delta \epsilon_{n}\right\}\right)
\end{array}
$$

Substituindo-se a expressão (5.71) na equação (5.70), tem-se na derivada do segundo termo de (5.68), a expressão:

$$
\left[\frac{\partial \Delta D_{n}}{\partial \Delta \epsilon_{n}}\right]=\frac{1}{M}[E]:\left(\left\{\epsilon_{n}\right\}+2\left\{\Delta \epsilon_{n}\right\}\right)
$$

Introduzindo-se o termo (5.72) na equação (5.68), tem-se o operador tangente consistente: 


$$
\left[\frac{\partial \Delta \sigma_{n}}{\partial \Delta \epsilon_{n}}\right]=\left(1-D_{n}-\Delta D_{n}\right)[E]-\frac{1}{M}\left\{E:\left(\epsilon_{n}+\Delta \epsilon_{n}\right)\right\} \otimes\left\{E:\left(\epsilon_{n}+2 \Delta \epsilon_{n}\right)\right\}
$$

sendo o produto tensorial representado pelo símbolo " $\otimes$ ". No limite, para $\Delta \epsilon_{n} \rightarrow 0$ a expressão (5.73) se transforma no operador tangente contínuo.

$$
\left[\frac{\partial \sigma}{\partial \epsilon}\right]=(1-D)[E]=-\frac{1}{M}\{E:(\epsilon)\} \otimes\{E:(\epsilon)\}
$$

\subsubsection{Modelo de Dano do Concreto}

O modelo de dano do concreto proposto neste trabalho é o modelo de (Comi ; Perego, 2000).Trata-se de um modelo com variáveis de dano para o caso de solicitações de tração $D_{t}$ e para solicitações de compressão $D_{c}$, sendo que essas variáveis variam de 1 até 0 . Em vista da não simetria do comportamento à tração e a compressão do concreto, definem-se duas funções distintas de carregamento, sendo $f_{t}$ para o caso de tração e $f_{c}$ para compressão. A energia livre $\Psi$, função das variáveis do estado, é dada por:

$$
\Psi\left(\epsilon, D_{t}, D_{c}\right)=\frac{1}{2}\left\{2 \mu_{0}\left(1-D_{t}\right)\left(1-D_{c}\right) \boldsymbol{e}: \boldsymbol{e}+K_{0}\left(\operatorname{tr} \epsilon^{+}\right)^{2}+K_{0}\left(\operatorname{tr} \epsilon^{-}\right)^{2}\right\} 5 .
$$

onde $\boldsymbol{\epsilon}$ é o tensor de deformações totais, $\boldsymbol{e}$ é a parte desviadora do tensor de deformações e $\mu_{0}$ e $K_{0}$ são respectivamente, o módulo de cisalhamento e o módulo de compressão volúmica (bulk elastic modulus), o qual é dado pela relação $K_{0}=2 G(1+\mu) /(3-2 \mu)$. Os valores de $\operatorname{tr} \epsilon^{+}$e $\operatorname{tr} \epsilon^{-}$se referem ao traço do tensor de deformações para o caso da deformação ser proveniente de tração ou compressão, respectivamente, e são dados por:

$$
\operatorname{tr} \epsilon^{+}=\langle\operatorname{tr} \epsilon\rangle
$$


$\operatorname{tr} \epsilon^{-}=-\langle-\operatorname{tr} \epsilon\rangle$

O tensor de tensão é obtido através de:

$$
\boldsymbol{\sigma}=\frac{\partial \Psi}{\partial \boldsymbol{\epsilon}}=2 \mu \boldsymbol{e}+\left(K_{+} \operatorname{tr} \epsilon^{+}\right) \boldsymbol{I}+\left(K_{-} \operatorname{tr} \epsilon^{-}\right) \boldsymbol{I}
$$

onde $\mu=\mu_{0}\left(1-D_{t}\right)\left(1-D_{c}\right)$, é o módulo de cisalhamento secante do material com dano e, $K_{+}=K_{0}\left(1-D_{t}\right)$ o módulo de compressão volúmica quando $\operatorname{tr} \epsilon^{+} \geq 0$, e $K_{-}=K_{0}\left(1-D_{c}\right)$ quando $\operatorname{tr} \epsilon^{-}<0$. $\boldsymbol{I}$ é o tensor identidade de segunda ordem. As funções de carregamento $f_{t}$ e $f_{c}$ podem ser escritas por:

$$
\begin{array}{r}
f_{t}=J_{2}-a_{t} I_{1}^{2}+b_{t} r_{t}\left(D_{t}\right) I_{1}-k_{t} r_{t}^{2}\left(D_{t}\right)\left(1-\alpha D_{c}\right) \\
f_{c}=J_{2}-a_{c} I_{1}^{2}+b_{c} r_{c}\left(D_{c}\right) I_{1}-k_{c} r_{c}^{2}\left(D_{c}\right)
\end{array}
$$

$$
r_{i}\left(D_{i}\right)=\left\{\begin{array}{cc}
1-\frac{1-\left(\frac{\sigma_{c}}{\sigma_{0}}\right)}{D_{0_{i}}^{2}}\left(D_{0_{i}}-D_{i}\right)^{2} & \text { para } D_{i}>D_{0_{i}} \\
{\left[1-\left(\frac{D_{i}-D_{0_{i}}}{1-D_{0_{i}}}\right)^{c_{i}}\right]^{0.75}} & \text { para } D_{i} \geq D_{0_{i}}
\end{array}\right.
$$

onde $J_{2}=\frac{1}{2} s: s$ é o segundo invariante do tensor $s$ da parte desviadora do tensor de tensões, $I_{1}=\operatorname{tr} \sigma$ é o primeiro invariante de $\sigma$, enquanto que, $a_{t}, b_{t}, k_{t}, a_{c}, b_{c}$, $k_{c}$ e $\alpha$ são parâmetros não negativos do material. Os termos $r_{t}\left(D_{t}\right)$ e $r_{c}\left(D_{c}\right)$ representam o trecho com endurecimento (hardening) que está expandindo e o trecho com amolecimento (softening) que está retraindo, como mostra a figura $(5.8) ; D_{0_{t}}$ e $D_{0_{c}}$ são os valores da variável de dano à tração e à compressão associados, respectivamente, aos valores $\sigma_{0_{t}}$ e $\sigma_{0_{c}}$ das tensões de pico, ou máximas, obtidos das 
curvas tensão-deformação em tração e compressão simples; $\sigma_{e_{t}}$ e $\sigma_{e_{c}}$ são as tensões do limite elásticos das mesmas curvas e $c_{t}>1$ e $c_{c}>1$ são parâmetros definindo as inclinações dos trechos com "softening". O termo $\left(1-\alpha D_{c}\right)$, da equação (5.49), permite considerar o decréscimo da capacidade de carga em tração pela existência anterior de dano à compressão.

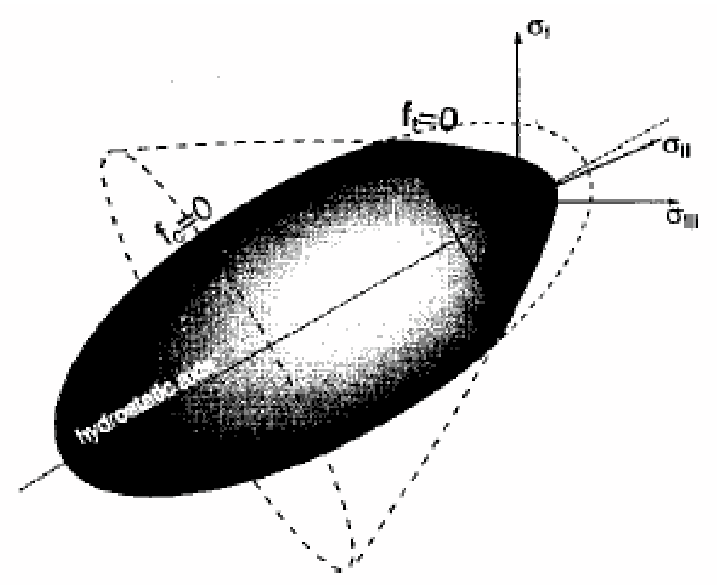

Fig. 5.8: Funções $f_{t}$ e $f_{c}$ definidas no espaço de tensões (Haigh-Westergaard). Figura retirada de $\operatorname{Comi}(2001)$

A figura (5.8) mostra as funções $f_{t}$ e $f_{c}$ onde a função $f_{t}$ é definida por um hiperbolóide e a função $f_{c}$ define uma elipsóide no mesmo espaço. O domínio elástico está dentro desse volume, podendo se expandir na fase de endurecimento e depois pode se retrair em função do dano por amolecimento. As condições de Kuhn-Tucker para a evolução do dano são dadas por:

$$
\begin{array}{lll}
f_{t} \leq 0, & \dot{D}_{t} \geq 0, & f_{t} \dot{D}_{t}=0 \\
f_{c} \leq 0, & \dot{D}_{c} \geq 0, & f_{c} \dot{D}_{c}=0
\end{array}
$$

As equações de $f_{t}$ e $f_{c}$ neste trabalho serão escritas em função da deformação:

$$
f_{t}\left(\epsilon, D_{t}, D_{c}\right)=4 \mu^{2} J_{\epsilon}-a_{t}\left(K_{+} \operatorname{tr}^{+} \epsilon+K_{-} \operatorname{tr}^{-} \epsilon\right)^{2}+
$$




$$
\begin{array}{r}
b_{t} r_{t}\left(D_{t}\right)\left(K_{+} \operatorname{tr}^{+} \epsilon+K_{-} \operatorname{tr}^{-} \epsilon\right)-k_{t} r_{t}^{2}\left(D_{t}\right)\left(1-\alpha D_{c}\right) \\
f_{c}\left(\epsilon, D_{t}, D_{c}\right)=4 \mu^{2} J_{\epsilon}-a_{c}\left(K_{+} \operatorname{tr}^{+} \epsilon+K_{-} \operatorname{tr}^{-} \epsilon\right)^{2}+ \\
b_{c} r_{c}\left(D_{c}\right)\left(K_{+} \operatorname{tr}^{+} \epsilon+K_{-} \operatorname{tr}^{-} \epsilon\right)-k_{c} r_{c}^{2}\left(D_{c}\right)
\end{array}
$$

\subsubsection{Processo não-linear incremental - Dano do Concreto}

Para o modelo de dano de concreto, tem-se, considerando-se as condições de Kuhn-Tucker:

$$
\begin{array}{r}
\boldsymbol{\sigma}_{\boldsymbol{n}+\mathbf{1}}=2 \mu_{0}\left(1-D_{t_{n+1}}\right)\left(1-D_{t_{n+1}}\right) \boldsymbol{e}_{\boldsymbol{n}+\mathbf{1}}+ \\
+K_{0}\left(1-D_{t_{n+1}}\right) \operatorname{tr}^{+} \epsilon_{n+1} \boldsymbol{I}+K_{0}\left(1-D_{c_{n+1}}\right) \operatorname{tr}^{-} \epsilon_{n+1} \boldsymbol{I} \text { em S(5.83) } \\
F_{t_{n+1}}=F_{t}\left(\epsilon_{n+1}, D_{t_{n+1}}, D_{c_{n+1}}\right) \leq 0 ; \Delta D_{t_{n}} \geq 0 ; \quad\left(F_{t_{n+1}} \Delta D_{t_{n}}\right)=0 \text { em S(5.84) } \\
F_{c_{n+1}}=F_{c}\left(\epsilon_{n+1}, D_{t_{n+1}}, D_{c_{n+1}}\right) \leq 0 ; \Delta D_{c_{n}} \geq 0 ; \quad\left(F_{c_{n+1}} \Delta D_{c_{n}}\right)=0 \text { em S(5.85) }
\end{array}
$$

onde $\Delta$ representa o incremento no passo de carga. Neste caso o tensor de incremento de tensão é dado por:

$$
\Delta \boldsymbol{\sigma}_{\boldsymbol{n}}=\tilde{\boldsymbol{E}}: \boldsymbol{\Delta} \boldsymbol{\epsilon}_{\boldsymbol{n}}-\boldsymbol{A} \Delta D_{t_{n}}-\boldsymbol{B} \Delta D_{c_{n}}
$$

onde:

$$
\begin{array}{r}
\tilde{\boldsymbol{E}}=2 \mu \boldsymbol{I} \otimes \boldsymbol{I}+\left[K_{+} H(\operatorname{tr} \epsilon)+K_{-} H(-\operatorname{tr} \epsilon)-\frac{2 \mu}{3}\right] \boldsymbol{I} \otimes \boldsymbol{I} \\
\boldsymbol{A}=2 \mu_{0}\left(1-D_{c}\right) \boldsymbol{e}+K_{0} \operatorname{tr}^{+} \epsilon \boldsymbol{I} \\
\boldsymbol{B}=2 \mu_{0}\left(1-D_{t}\right) \boldsymbol{e}+K_{0} \operatorname{tr}^{-} \epsilon \boldsymbol{I}
\end{array}
$$


onde $\tilde{\boldsymbol{E}}$ é o tensor elástico constitutivo do material com dano, $\boldsymbol{I} \bar{\otimes} \boldsymbol{I}$ é o tensor de identidade simétrico de quarta ordem com componentes $i j k l$ dadas por $1 / 2\left(\delta_{i k} \delta_{j l}+\right.$ $\left.\delta_{i l} \delta_{j k}\right), \boldsymbol{I} \otimes \boldsymbol{I}$ o tensor com componentes $\delta_{i j} \delta_{k l}, H(x)$ é a função de Heavyside que assume valor unitário para $x \geq 0$ e zero para $x<0$.

Derivando-se a equação (5.86) em relação a incrementos de deformações, temse:

$$
\frac{\partial \Delta \sigma_{n}}{\partial \Delta \epsilon_{n}}=\tilde{E}-\left[A \otimes \frac{\partial D_{t_{n}}}{\partial \Delta \epsilon_{n}}\right]-\left[B \otimes \frac{\partial D_{c_{n}}}{\partial \Delta \epsilon_{n}}\right]
$$

A evolução das variáveis de dano $\Delta D_{t_{n}} \mathrm{e} / \mathrm{ou} \Delta D_{c_{n}}$ está condicionada às seguintes condições:

$$
\begin{aligned}
& \dot{f}_{t}=\frac{\partial \boldsymbol{f}_{\boldsymbol{t}}}{\boldsymbol{\partial \boldsymbol { \epsilon }}}: \dot{\boldsymbol{\epsilon}}+\frac{\partial f_{t}}{\partial D_{t}} \dot{D}_{t}+\frac{\partial f_{t}}{\partial D_{c}} \dot{D}_{c}=0 \\
& \dot{f}_{c}=\frac{\boldsymbol{\partial \boldsymbol { f } _ { \boldsymbol { c } }}}{\boldsymbol{\partial \boldsymbol { \epsilon }}}: \dot{\boldsymbol{\epsilon}}+\frac{\partial f_{c}}{\partial D_{t}} \dot{D}_{t}+\frac{\partial f_{c}}{\partial D_{c}} \dot{D}_{c}=0
\end{aligned}
$$

em forma incremental:

$$
\begin{aligned}
& \Delta f_{t_{n}}=\frac{\partial \Delta f_{t_{n}}}{\partial \Delta \epsilon_{\boldsymbol{n}}}: \Delta \boldsymbol{\epsilon}_{\boldsymbol{n}}+\frac{\partial \Delta f_{t_{n}}}{\partial \Delta D_{t_{n}}} \Delta D_{t_{n}}+\frac{\partial \Delta f_{t_{n}}}{\partial \Delta D_{c_{n}}} \Delta D_{c_{n}}=0 \\
& \Delta f_{c_{n}}=\frac{\boldsymbol{\partial} \boldsymbol{\Delta} \boldsymbol{f}_{c_{n}}}{\boldsymbol{\partial} \boldsymbol{\Delta} \boldsymbol{\epsilon}_{\boldsymbol{n}}}: \Delta \boldsymbol{\epsilon}_{\boldsymbol{n}}+\frac{\partial \Delta f_{c_{n}}}{\partial \Delta D_{t_{n}}} \Delta D_{t_{n}}+\frac{\partial \Delta f_{c_{n}}}{\partial \Delta D_{c_{n}}} \Delta D_{c_{n}}=0
\end{aligned}
$$

sendo por relação:

$$
\frac{\partial \boldsymbol{f}_{\boldsymbol{t}}}{\boldsymbol{\partial \boldsymbol { \epsilon }}}=\frac{\boldsymbol{\Delta} \boldsymbol{f}_{\boldsymbol{t}_{n}}}{\boldsymbol{\partial \Delta \boldsymbol { \epsilon } _ { \boldsymbol { n } }} ;} \quad \frac{\partial f_{t}}{\partial D_{t}}=\frac{\partial \Delta f_{t_{n}}}{\partial \Delta D_{t_{n}}} ; \quad \frac{\partial f_{t}}{\partial D_{c}}=\frac{\partial \Delta f_{t_{n}}}{\partial \Delta D_{c_{n}}}
$$




$$
\frac{\partial f_{c}}{\partial \boldsymbol{\epsilon}}=\frac{\Delta f_{c_{n}}}{\partial \Delta \boldsymbol{\epsilon}_{\boldsymbol{n}}} ; \quad \frac{\partial f_{c}}{\partial D_{t}}=\frac{\partial \Delta f_{c_{n}}}{\partial \Delta D_{t_{n}}} ; \quad \frac{\partial f_{c}}{\partial D_{c}}=\frac{\partial \Delta f_{c_{n}}}{\partial \Delta D_{c_{n}}}
$$

sendo que para cada iteração, tem-se os seguintes intervalos:

$$
\begin{array}{r}
\Delta f_{t_{n}}^{i}=f_{t_{n+1}}^{i}-f_{t_{n}}, \quad \Delta f_{c_{n}}^{i}=f_{c_{n+1}}^{i}-f_{c_{n}} \\
\Delta \boldsymbol{\epsilon}_{\boldsymbol{n}}^{\boldsymbol{i}}=\boldsymbol{\epsilon}_{\boldsymbol{n + 1}}^{\boldsymbol{i}}-\boldsymbol{\epsilon}_{\boldsymbol{n}}, \quad \Delta D_{t_{n}}^{i}=D_{t_{n+1}}^{i}-D_{t_{n}}, \quad \Delta D_{c_{n}}^{i}=D_{c_{n+1}}^{i}-D_{c_{n}}(5.94)
\end{array}
$$

ou seja, a derivada em relação a uma variável incremental é igual à derivada em relação à variável total equivalente, pois num instante $n$, como mostram as equações (5.94), uma é igual à outra a menos de uma constante.

As duas equações (5.92) formam um sistema linear com variáveis em $\Delta D_{t_{n}} \mathrm{e}$ $\Delta D_{c_{n}}$. Isolando tais termos, tem-se a expressão em função de $\boldsymbol{\Delta} \boldsymbol{\epsilon}_{\boldsymbol{n}}$.

$$
\begin{aligned}
\Delta D_{t_{n}}= & \frac{\left(\frac{\partial \Delta f_{t_{n}}}{\partial \Delta D_{c_{n}}} \frac{\partial \Delta f_{c_{n}}}{\partial \Delta \epsilon_{n}}: \Delta \epsilon_{n}-\frac{\partial \Delta f_{c_{n}}}{\partial \Delta D_{c_{n}}} \frac{\partial \Delta f_{t_{n}}}{\partial \Delta \epsilon_{n}}: \Delta \epsilon_{n}\right)}{h} \\
\Delta D_{c_{n}}= & \frac{\left(\frac{\partial \Delta f_{c_{n}}}{\partial \Delta D_{t_{n}}} \frac{\partial \Delta f_{t_{n}}}{\partial \Delta \epsilon_{n}}: \Delta \epsilon_{n}-\frac{\partial \Delta f_{t_{n}}}{\partial \Delta D_{t_{n}}} \frac{\partial f_{c_{n}}}{\partial \Delta \epsilon_{n}}: \Delta \epsilon_{n}\right)}{h}
\end{aligned}
$$

sendo $h$ o escalar dado por:

$$
h=\frac{\partial \Delta f_{t_{n}}}{\partial \Delta D_{t_{n}}} \frac{\partial \Delta f_{c_{n}}}{\partial \Delta D_{c_{n}}}-\frac{\partial \Delta f_{t_{n}}}{\partial \Delta D_{c_{n}}} \frac{\partial \Delta f_{c_{n}}}{\partial \Delta D_{t_{n}}}
$$

As derivadas (5.95) e (5.96) em relação ao tensor de deformações, resultam em: 


$$
\begin{aligned}
\frac{\partial \Delta D_{t_{n}}}{\partial \Delta \epsilon_{n}}= & {\left[\frac{\partial \Delta f_{t_{n}}}{\partial \Delta D_{c_{n}}}\left(\frac{\partial^{2} \Delta f_{c_{n}}}{\partial^{2} \Delta \epsilon_{n}}: \Delta \epsilon_{n}+\frac{\partial \Delta f_{c_{n}}}{\partial \Delta \epsilon_{n}}\right)+\right.} \\
& \left.-\frac{\partial \Delta f_{c_{n}}}{\partial \Delta D_{c_{n}}}\left(\frac{\partial^{2} \Delta f_{t_{n}}}{\partial^{2} \Delta \epsilon_{n}}: \Delta \epsilon_{n}+\frac{\partial \Delta f_{t_{n}}}{\partial \Delta \epsilon_{n}}\right)\right] \\
\frac{\partial \Delta D_{c_{n}}}{\partial \Delta \epsilon_{n}}= & {\left[\frac{\partial \Delta f_{c_{n}}}{\partial \Delta D_{t_{n}}}\left(\frac{\partial^{2} \Delta f_{t_{n}}}{\partial^{2} \Delta \epsilon_{n}}: \Delta \epsilon_{n}+\frac{\partial \Delta f_{t_{n}}}{\partial \Delta \epsilon_{n}}\right)+\right.} \\
& \left.-\frac{\partial \Delta f_{t_{n}}}{\partial \Delta D_{t_{n}}}\left(\frac{\partial^{2} \Delta f_{c_{n}}}{\partial^{2} \Delta \epsilon_{n}}: \Delta \epsilon_{n}+\frac{\partial \Delta f_{c_{n}}}{\partial \Delta \epsilon_{n}}\right)\right]
\end{aligned}
$$

Substituindo-se as expressões (5.98) e (5.99) na equação (5.90), obtém-se o operador tangente consistente para o modelo de dano do concreto.

$$
\begin{array}{r}
\frac{\partial \Delta \sigma_{n}}{\partial \Delta \epsilon_{n}}=\tilde{E}-\frac{1}{h}\left\{\boldsymbol { A } \otimes \left[\frac{\partial \Delta f_{t_{n}}}{\partial \Delta D_{c_{n}}}\left(\frac{\partial^{2} \Delta f_{c_{n}}}{\partial^{2} \Delta \epsilon_{n}}: \Delta \epsilon_{n}+\frac{\partial \Delta f_{c_{n}}}{\partial \Delta \epsilon_{n}}\right)-\frac{\partial \Delta f_{c_{n}}}{\partial \Delta D_{c_{n}}}\right.\right. \\
\left.\left(\frac{\partial^{2} \Delta f_{t_{n}}}{\partial^{2} \Delta \epsilon_{n}}: \Delta \epsilon_{n}+\frac{\partial \Delta f_{t_{n}}}{\partial \Delta \epsilon_{n}}\right)\right]+B \otimes\left[\frac { \partial \Delta f _ { c _ { n } } } { \partial \Delta D _ { t _ { n } } } \left(\frac{\partial^{2} \Delta f_{t_{n}}}{\partial^{2} \epsilon_{n}}: \Delta \epsilon_{n}+\right.\right. \\
\left.\left.\left.+\frac{\partial \Delta f_{t_{n}}}{\partial \Delta \epsilon_{n}}\right)-\frac{\partial \Delta f_{t_{n}}}{\partial \Delta D_{t_{n}}}\left(\frac{\partial^{2} \Delta f_{c_{n}}}{\partial^{2} \Delta \epsilon_{n}}: \Delta \epsilon_{n}+\frac{\partial \Delta f_{c_{n}}}{\partial \Delta \epsilon_{n}}\right)\right]\right\}
\end{array}
$$

As operações tensoriais da equação (5.100) resultam em tensores de quarta ordem, pois $\boldsymbol{A}$ e $\boldsymbol{B}$ e os tensores em colchete são de segunda ordem. Fazendo $\boldsymbol{\Delta} \boldsymbol{\epsilon}_{\boldsymbol{n}}$ tender a zero, a equação (5.100) se transforma no operador tangente contínuo do modelo.

$$
\frac{\boldsymbol{\partial} \boldsymbol{\sigma}}{\boldsymbol{\partial} \boldsymbol{\epsilon}}=\tilde{\boldsymbol{E}}-\frac{1}{h}\left[\boldsymbol{A} \otimes\left(\frac{\partial f_{t}}{\partial D_{c}} \frac{\partial \boldsymbol{f}_{\boldsymbol{c}}}{\boldsymbol{\partial} \boldsymbol{\epsilon}}-\frac{\partial f_{c}}{\partial D_{c}} \frac{\boldsymbol{\partial \boldsymbol { f } _ { \boldsymbol { t } }}}{\boldsymbol{\partial \boldsymbol { \epsilon }}}\right)+\boldsymbol{B} \otimes\left(\frac{\partial f_{c}}{\partial D_{t}} \frac{\boldsymbol{\partial \boldsymbol { f } _ { \boldsymbol { t } }}}{\boldsymbol{\partial} \boldsymbol{\epsilon}}-\frac{\partial f_{t}}{\partial D_{t}} \frac{\boldsymbol{\partial \boldsymbol { f } _ { \boldsymbol { c } }}}{\boldsymbol{\partial} \boldsymbol{\epsilon}}\right)\right]
$$

voltando-se, com a equação (5.101) ao operador tangente consistente da equação de equilíbrio em deformações das formulações não-lineares, onde um de seus termos é $\partial \Delta \sigma_{n} / \partial \Delta \epsilon_{n}$ 


\subsection{Localização do Dano dando início à Fratura}

Uma das dificuldades da mecânica da fratura é de se determinar como se dá o início e a propagação do processo de fraturamento. Em materiais cimentícios, como o concreto, considera-se que o início da fratura se dê pela junção de pequenas microfissuras, que se dão entre os constituintes do material.

Considera-se neste trabalho, como na seção (4.1.1) do capítulo 4, que em uma sub-região muito fina, o material começa a se degenerar segundo a lei de dano simples. Tal procedimento visa a simulação do início de fraturamento do sólido, pois o início do processo de fraturamento, como foi discutido anteriormente, se dá pela junção de pequenas microfissuras as quais são simuladas pela mecânica do dano.

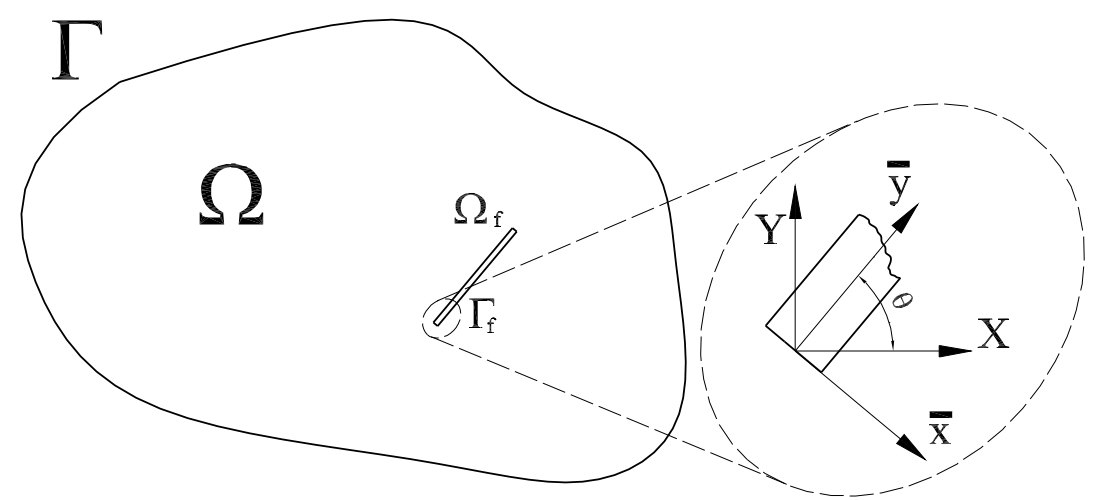

Fig. 5.9: Orientação dos eixos locais da sub-região de origem da fratura

Neste trabalho, adotou-se a hipótese, até pela característica geométrica de uma região de fratura, que a direção de propagação das degenerações da rigidez do domínio se dê pela orientação dos eixos locais, como mostra a figura (5.9), pois deve-se considerar neste caso principalmente a variação da abertura da fratura $\Delta u_{n}$, na direção normal ao comprimento da sub-região, e a distorção entre as faces da fratura na direção do comprimento, $\Delta u_{s}$, como um comportamento mecânico de uma região de fratura, tendo como matriz de rotação de eixos $X-Y$ para os eixos locais da fratura $\bar{x}-\bar{y}$, a equação: 


$$
\boldsymbol{R}=\left[\begin{array}{ccc}
\operatorname{sen}^{2} \theta & -2 \cos \theta \operatorname{sen} \theta & \cos ^{2} \theta \\
\cos \theta \operatorname{sen} \theta & \left(\operatorname{sen}^{2} \theta-\cos ^{2} \theta\right) & -\operatorname{sen} \theta \cos \theta \\
\cos ^{2} \theta & 2 \cos \theta \operatorname{sen} \theta & \operatorname{sen}^{2} \theta
\end{array}\right]
$$

Com isso, utilizando-se a expressão (5.102) na expressão (5.37) tem-se as expressões :

$$
\left\{\bar{\sigma}^{e}\right\}=[R]\{N\}+[R][\bar{S}]\left\{\bar{\sigma}_{0}\right\}
$$

onde os termos de tensões iniciais $\bar{\sigma}_{0}$ são escritos segundo orientação dos eixos locais $\bar{x}-\bar{y}$. Sendo válido no eixo local a relação $\left\{\bar{\sigma}^{e}\right\}=\{\bar{\sigma}\}+\left\{\bar{\sigma}^{0}\right\}$, tem-se a formulação constitutiva não linear escrita em termos de deformações locais:

$$
\{Y(\bar{\epsilon})\}=[E]\{\bar{\epsilon}\}+[R]\{N\}+[R][\bar{S}]\{[E]\{\bar{\epsilon}\}-\{\bar{\sigma}(\bar{\epsilon})\}\}=0
$$

Como mostra a figura (5.10), as deformações $\bar{\epsilon}_{x}$ e $\bar{\epsilon}_{x y}$ podem ser relacionadas com a variação dos deslocamentos através da espessura $h$ da fratura. Tal comparação é possível pois a espessura da fratura é muito pequena. Tem-se então a expressão:

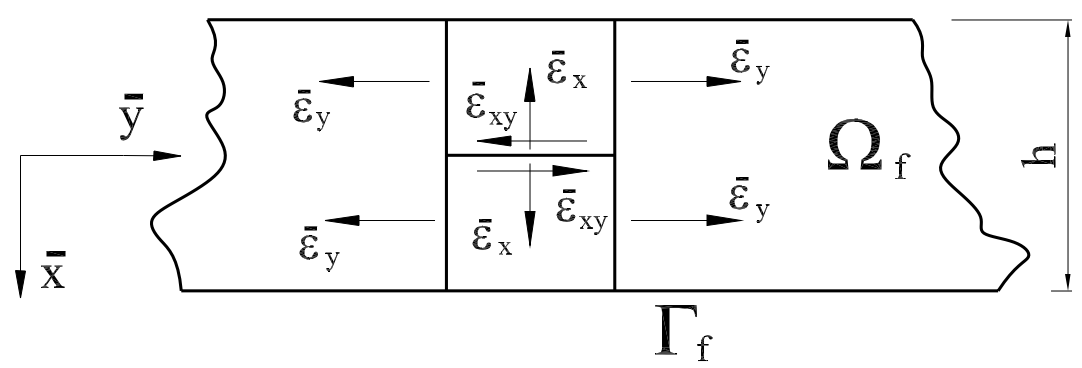

Fig. 5.10: Deslocamentos relativos entre a espessura da fratura 


$$
\bar{\epsilon}_{x}=\frac{\delta \bar{u}}{h} \quad \bar{\epsilon}_{x y}=\frac{\delta \bar{v}}{h}
$$

onde $\delta \bar{u}$ e $\delta \bar{u}$ são as variações dos deslocamentos lineares nas direções $\bar{x}$ e $\bar{y}$ respectivamente. Adotou-se $\delta$ como a variação de deslocamentos nas direções locais, para não haver confusão com o símbolo da variação das variáveis em análise não-linear.

O vetor de deformações locais $\{\bar{\epsilon}\}$ pode ser escrito em função da variação dos deslocamentos locais através da espessura $h$.

$$
\left\{\begin{array}{c}
\bar{\epsilon}_{x} \\
\bar{\epsilon}_{x y} \\
\bar{\epsilon}_{y}
\end{array}\right\}=\left[\begin{array}{ccc}
1 / h & 0 & 0 \\
0 & 1 / h & 0 \\
0 & 0 & 1
\end{array}\right]\left\{\begin{array}{l}
\delta \bar{u} \\
\delta \bar{v} \\
\bar{\epsilon}_{y}
\end{array}\right\}
$$

onde pode-se chamar de matriz $[T]$ os termos da espessura $h$ e, o vetor $\bar{z}$, como o vetor em termo de deslocamentos e deformações incógnitas da fratura.

$$
[T]=\left[\begin{array}{ccc}
1 / h & 0 & 0 \\
0 & 1 / h & 0 \\
0 & 0 & 1
\end{array}\right] \quad\{\bar{z}\}=\left\{\begin{array}{l}
\delta \bar{u} \\
\delta \bar{v} \\
\bar{\epsilon}_{y}
\end{array}\right\}
$$

Utilizando-se as expressões (5.107) na expressão (5.104) tem-se:

$$
\{Y(\bar{z})\}=[E][T]\{\bar{z}\}+[R]\{N\}+[R][\bar{S}]\{[E][T]\{\bar{z}\}-\{\bar{\sigma}(\bar{z})\}\}=0
$$

a qual pode ser escrita na forma incremental, para resolução por processo não-linear de passo de carga. 


$$
\{Y(\Delta \bar{z})\}=[E][T]\{\Delta \bar{z}\}+[R]\{\Delta N\}+[R][\bar{S}]\{[E][T]\{\Delta \bar{z}\}-\{\Delta \bar{\sigma}(\Delta \bar{z})\}\}=0
$$

\subsubsection{Processo não linear incremental}

Utilizando-se do processo tipo Newton-Raphson não linear iterativo, com fases de previsão e correção, tem-se para cada iteração:

$$
\left\{\bar{z}_{n}^{i+1}\right\}=\left\{\Delta \bar{z}_{n}^{i}\right\}+\left\{\delta \Delta \bar{z}_{n}^{i+1}\right\}
$$

Expandindo-se a expressão (5.109) em uma série de Taylor e, considerando apenas o primeiro termo da série, tem-se:

$$
Y\left(\left\{\Delta \bar{z}_{n}^{i}\right\}\right)=\frac{\partial\left\{Y\left(\left\{\Delta \bar{z}_{n}^{i}\right\}\right)\right\}}{\partial\left\{\Delta \bar{z}_{n}^{i}\right\}}\left\{\delta \Delta \bar{z}_{n}^{i}\right\}+\cdots=0
$$

utilizando-se o primeiro termo da equação (5.111) e derivando a expressão , tem-se:

$$
\left\{Y\left(\Delta \bar{z}_{n}^{i}\right)\right\}=[E][T]-[R][\bar{S}]\left\{[E][T]-\left[\frac{\partial \Delta \bar{\sigma}_{n}^{i}}{\partial \Delta \bar{z}_{n}^{i}}\right]\right\}\left\{\delta \Delta \bar{z}_{n}^{i}\right\}
$$

onde o operador que multiplica $\left\{\delta \Delta \bar{z}_{n}^{i}\right\}$ é o operador tangente contínuo na direção dos eixos locais da fratura.

Escrevendo-se as variáveis incrementais de tensões, a equação (5.67) pode ser escrita em variáveis locais.

$$
\Delta \bar{\sigma}_{n}=\left(1-D_{n}-\Delta D_{n}\right)[E][T]:\left\{\Delta \bar{z}_{n}\right\}-\Delta D_{n}[E][T]:\left\{\Delta \bar{z}_{n}\right\}
$$


onde a matriz tangente consistente é dada pela derivada:

$$
\left[\frac{\partial \Delta \bar{\sigma}_{n}}{\partial \Delta \bar{z}_{n}}\right]=\left(1-D_{n}-\Delta D_{n}\right)[E][T]-\left[[E][T]:\left\{\bar{z}_{n}+\Delta \bar{z}_{n}\right\}\right] \otimes\left[\frac{\partial \Delta D_{n}}{\partial \Delta \bar{z}_{n}}\right](5
$$

Utilizando-se do último termo das equações (5.69) mas derivando as funções em relação as variáveis de deslocamentos locais $\Delta \bar{z}_{n}$ tem-se:

$$
\Delta f_{n}=\frac{\partial \Delta f_{n}}{\partial \Delta Y_{n}} \frac{\partial \Delta Y_{n}}{\partial \Delta \bar{z}_{n}} \Delta \bar{z}_{n}+\frac{\partial \Delta f_{n}}{\partial \Delta D_{n}} \Delta D_{n}=0
$$

onde isolando-se os termos de incrementos de dano:

$$
\Delta D_{n}=-\frac{\frac{\partial \Delta f_{n}}{\partial \Delta Y_{n}} \frac{\partial \Delta Y_{n}}{\partial \Delta \bar{z}_{n}}}{\frac{\partial \Delta f_{n}}{\partial \Delta D_{n}}}=\frac{\frac{\partial \Delta Y_{n}}{\partial \Delta \bar{z}_{n}}}{M} \Delta \bar{z}_{n}
$$

Escrevendo-se as equações de dano simples em função das variáveis locais de deslocamentos, tem-se:

$$
\begin{array}{r}
Y=\frac{1}{2}[T]\{\bar{z}\}:[E]:[T]\{\bar{z}\} \\
\Delta Y=[T]\{\bar{z}\}:[E]:[T]\{\Delta \bar{z}\}+\frac{1}{2}[T]\{\Delta \bar{z}\}:[E]:[T]\{\Delta \bar{z}\} \\
\frac{\partial \Delta Y}{\partial \Delta \bar{z}_{n}}=[E][T]^{2}:\left\{\bar{z}_{n}+\Delta \bar{z}_{n}\right\}
\end{array}
$$

Com a equação (5.117) substituída na expressão (5.116), a derivada do segundo termo de (5.114) pode ser escrita como: 


$$
\left[\frac{\partial \Delta D_{n}}{\partial \Delta \bar{z}_{n}}\right]=\frac{1}{M}[E][T]^{2}\left\{\bar{z}_{n}+2 \Delta \bar{z}_{n}\right\}
$$

Introduzindo o termo (5.118) na equação (5.114) tem-se o operador tangente consistente em função das variáveis de deslocamentos locais ao eixo da fratura.

$$
\begin{array}{r}
{\left[\frac{\partial \Delta \bar{\sigma}_{n}}{\partial \Delta \bar{z}_{n}}\right]=\left(1-D_{n}-\Delta D_{n}\right)[E][T]-\frac{1}{M}[T]\left[[E][T]:\left\{\bar{z}_{n}+\Delta \bar{z}_{n}\right\}\right] \otimes} \\
{\left[[E][T]:\left\{\bar{z}_{n}+2 \Delta \bar{z}_{n}\right\}\right]}
\end{array}
$$

No limite, para $\Delta \bar{z}_{n} \rightarrow 0$ tem-se o operador tangente contínuo:

$$
\left[\frac{\partial \bar{\sigma}}{\partial \bar{z}}\right]=(1-D)[E][T]-\frac{1}{M}[T]\left[[E][T]:\left\{\bar{z}_{n}\right\}\right] \otimes\left[[E][T]:\left\{\bar{z}_{n}\right\}\right]
$$

\subsection{Análise Numérica}

Na presente seção se faz uma análise numérica da vários modelos não lineares estudados neste capítulo.

\subsubsection{Modelo de Dano escalar}

O exemplo a seguir mostra uma viga biapoiada sujeita a um carregamento distribuído transversal. A figura (5.11) mostra as características geométricas e materiais da viga, bem como a discretização em células e a colocação dos nós de contorno e dos nós internos.

Nota-se na figura (5.11), o deslocamento para dentro da célula dos nós das mesmas que pertencem ao contorno, procedimento utilizado para se evitar singularidades nas equações fundamentais de tensão. 


\section{$\mathrm{E}=2100000 \mathrm{KPa}$} $\mathrm{v}=0,25$

$2000 \mathrm{kN} / \mathrm{m}$

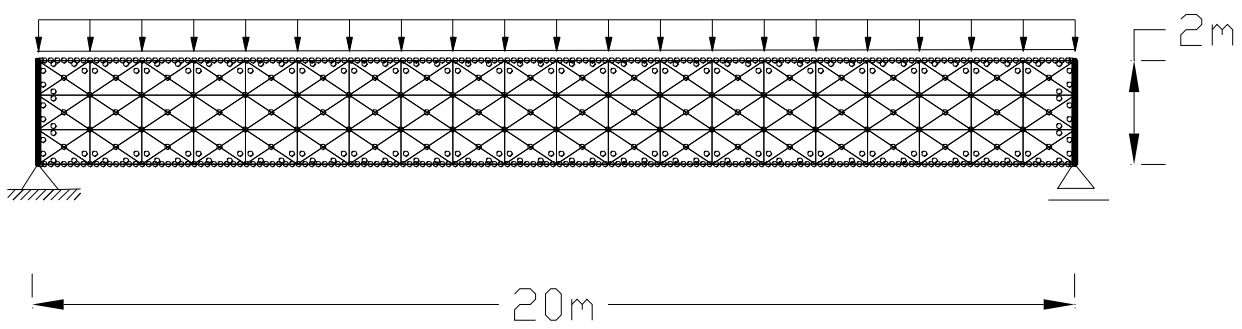

Fig. 5.11: Características geométricas e discretização

No modelo de dano escalar foram adotadas as constantes do modelo de dano simples, $M=0,025 k N / m^{2}$ e $Y_{0}=0,000166 k N / m^{2}$. A viga foi discretizada em 640 nós externos e 273 nós internos, onde as tensões e deformações foram aproximandos por 240 células internas. Na discretização do contorno foram utilizados 64 nós sendo 16 em cada face da viga.

O carregamento foi aplicado em incrementos, sendo que, cada incremento corresponde à milésima parte do carregamento total.

A figura (5.12) mostra a evolução do dano quando foram aplicados 6 incrementos de carga.

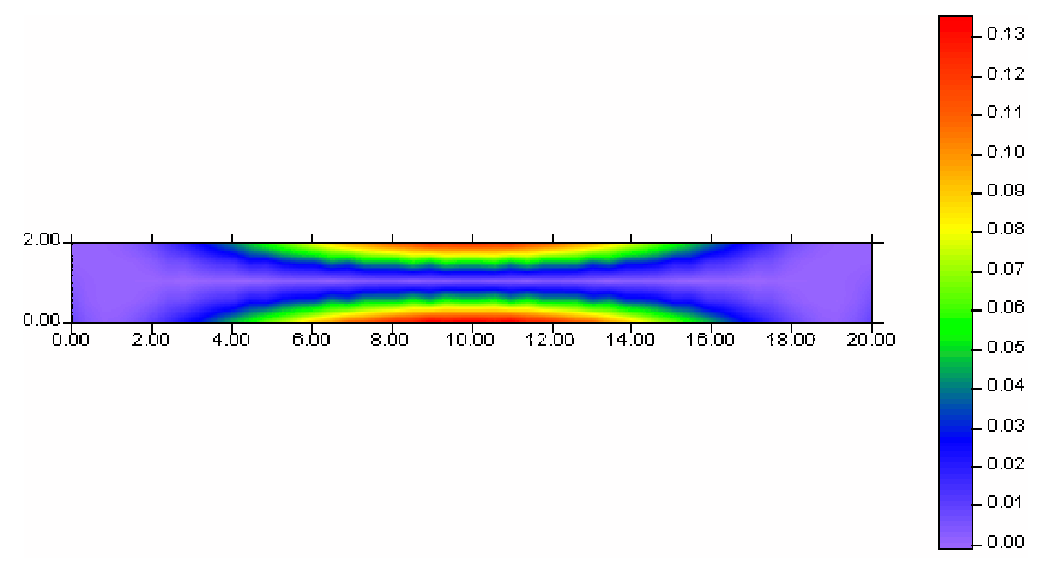

Fig. 5.12: Dano correspondente a 6 incrementos de carga 
Como pode ser notado na figura (5.12), o modelo de dano se mostra bastante preciso, coincidindo a maior danificação nas regiões mais solicitadas a tração e a compressão. A danificação é a mesma nas duas regiões, pois o modelo é o mesmo tanto para tração como para compressão. O exemplo apresentou uma danificação acentuada devido ao fato da tensão limite elástica adotada neste exemplo ser de 82 $k N / m^{2}$, bem inferior ao apresentado no meio da viga.

\subsubsection{Modelo de Dano do concreto}

O exemplo a seguir mostra uma viga biapoiada sujeita a um carregamento distribuído transversal idêntico à figura (5.11), sendo também adotada a mesma discretização e a mesmas características mecânicas. Neste caso o material vai sofrer degeneração segundo os critérios de danificação do modelo de concreto. O presente exemplo tem como características do modelo de dano do concreto: $a_{t}=0.31, a_{c}=$ $0.0025, b_{t}=4.0 \mathrm{kPa}, b_{c}=3.0 \mathrm{kPa}, \alpha=1.0, D_{0 t}=0.1, D_{0 c}=0.3, \sigma_{e}=10.0 \mathrm{kPa}, c_{t}=$ $5.0, c_{c}=5.0, \sigma_{0 t}=12.50 k P a, \sigma_{0 c}=14.28 k P a, k_{t}=8.0 k P a^{2}$ e $k_{c}=370.0 k P a^{2}$.

A figura (5.13) mostra a evolução do dano de tração quando foram aplicados 7 incrementos de carga.

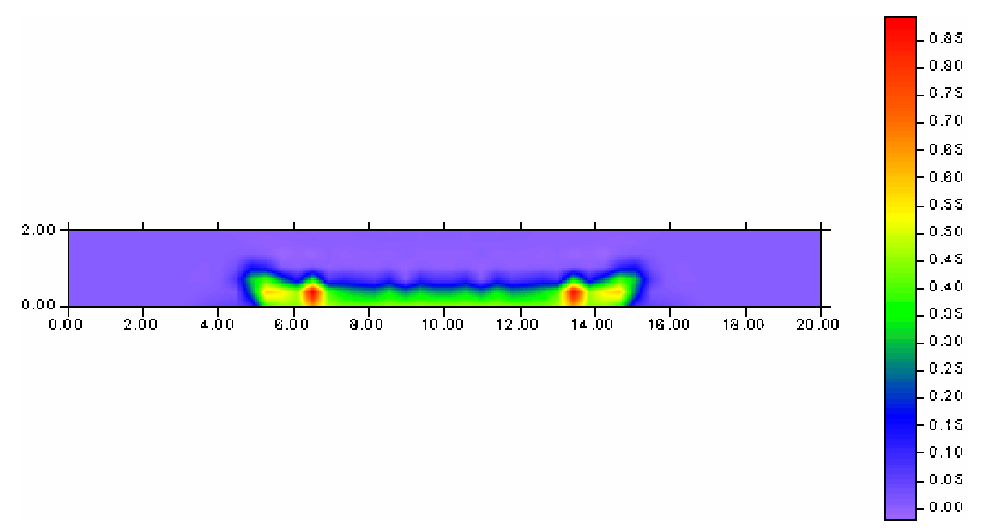

Fig. 5.13: Dano na tração ' $D_{t}$ 'correspondente a 7 incrementos de carga

O modelo de dano do concreto aprsentou também resultados bastante coerentes. A região com maior danificação à tração ocorreu aproximadamente a um terço dos apoios. Isto se deve ao fato da combinação das tensões normais de tração e das tensões cisalhantes, pois no meio da viga, apesar das tensões normais serem 
máximas, não há na extremidade inferior da viga tensão cisalhante.

\subsubsection{Degeneração de dano simples originando fratura}

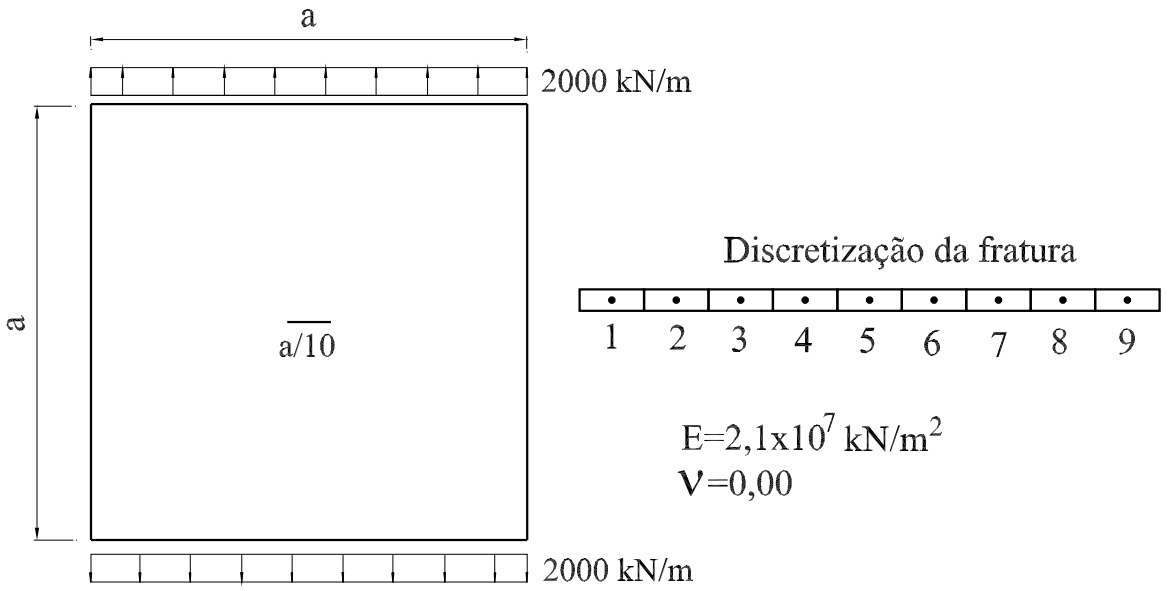

Fig. 5.14: Sólido com sub-região de origem de fratura

A figura (5.14) mostra um sólido sujeito a carregamento de tração e uma estreita sub-região, onde se dará o processo de danificação que dará origem a uma fratura.

O sólido foi discretizado com 15 elementos lineares de contorno em cada face, num total de 64 nós no contorno. A sub-região central foi discretizada em 9 elementos de domínio, como mostra a figura (5.14), com aproximação constante. Foram feitas análises variando a espessura da fratura de $2,0 \cdot 10^{-4} \mathrm{~m}$ a $2,0 \cdot 10^{-6} \mathrm{~m}$, para se avaliar a influência da espessura da região a ser degenerada nos resultados. O valor de $a$ neste exemplo é de $20 \mathrm{~m}$. As características geométricas, do carregamento e do material estão descritos na figura (5.14). As características de dano simples são de $M=0,025 k N / m^{2}$ e $Y_{0}=0,0001667 k N / m^{2}$.

A seguir serão mostradas a evolução da tensão $\bar{\sigma}_{x}$, que é a tensão perpendicular ao comprimento da sub-região a ser degenerada com a evolução de $\delta \bar{u}$, ou seja, o deslocamento relativo no nó central no início da abertura da fratura. 


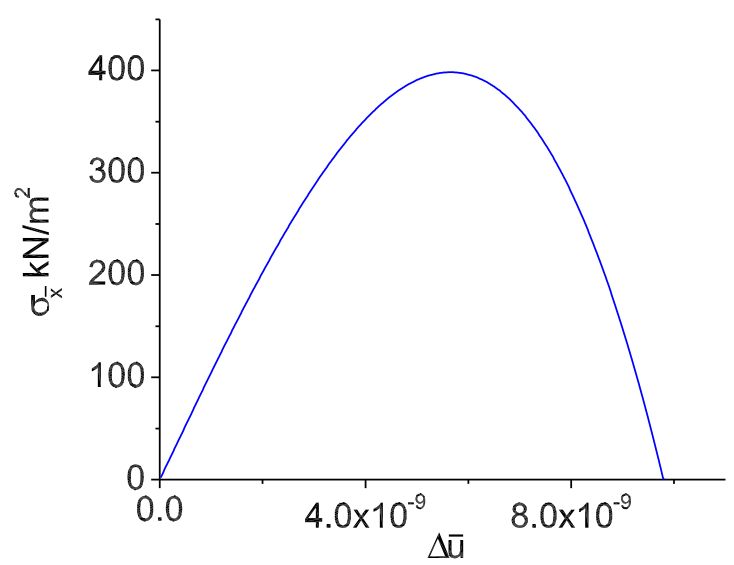

Fig. 5.15: Tensão $\sigma_{\bar{x}} \mathrm{X} \Delta \bar{u}$ para $\mathrm{h}=2 \times 10^{-4}$

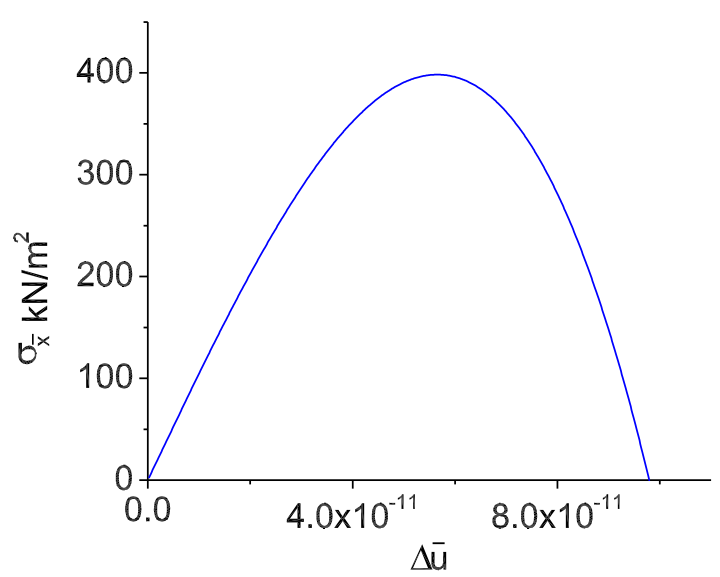

Fig. 5.16: Tensão $\sigma_{\bar{x}} \mathrm{X} \Delta \bar{u}$ para $\mathrm{h}=2 \times 10^{-6}$ 
A figura (5.17) mostra a variação do deslocamento na extensão ao longo da sub-região degenerada quando o dano é zero, isto é, a sub-região central não tem mais rigidez nenhuma.

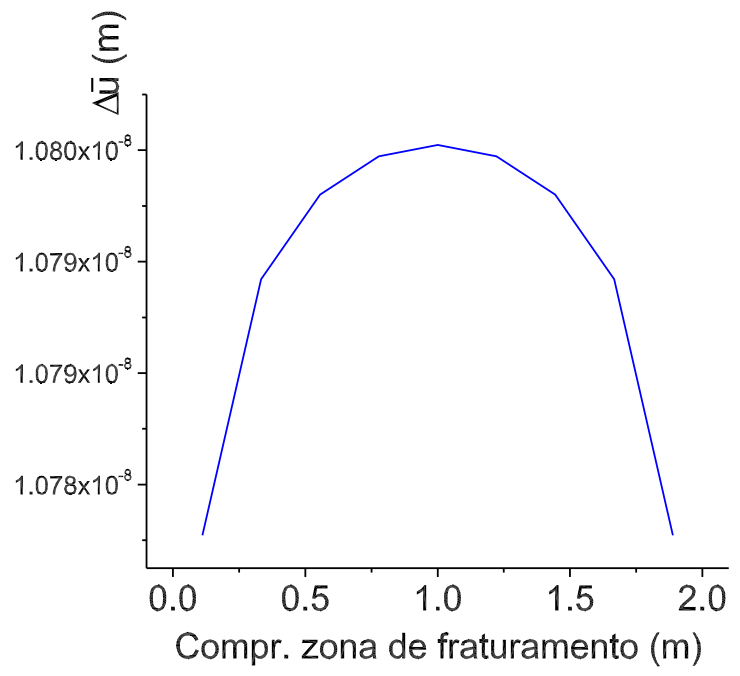

Fig. 5.17: Abertura $\Delta \bar{u}$ ao longo da fratura para $\mathrm{h}=2 \times 10^{-4}$

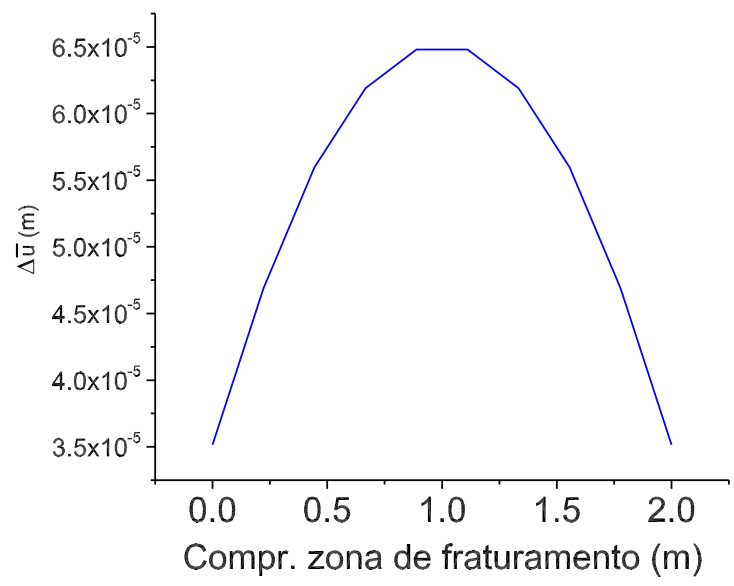

Fig. 5.18: Abertura da fratura em um modelo discreto

Em seguida é feita uma análise elástica, onde a sub-região agora é uma região discreta com uma rigidez quase nula. A discretização da malha e as características 
mecânicas do sólido, bem como as condições de contorno são as mesmas para a análise não linear.

Nota-se, nesse exemplo, que análise de dano para um sub-região muito esbelta apresenta diferenças significativas quanto a espessura desta região. Isso se deve ao fato do modelo partir da análise de deformação, que é uma taxa e por isso permanece inalterada independentemente da espessura da sub-região. Contudo, o modelo de dano aplicado a todo o sólido, se mostra viável na demarcação de regiões passíveis de fraturamento. Onde a danificação for mais acentuada, pode-se introduzir nestas regiões elementos que simulem uma fratura como os elementos discretos, analisados neste caso, ou elementos de formulação dual. 


\section{CONSIDERAÇÕES FINAIS}

Neste trabalho foram abordadas propostas teóricas e modelos numéricos, utilizando-se do Método dos Elementos de Contorno para o estudo de regiões de elasticidade plana constituídas por multidomínios. Foram consideradas regiões com inclusões e, inclusões muito delgadas que também foram analisadas com rigidez quase nula, para se chegar a um comportamento de fratura elástica. Na área de análise não linear, foi estudada a degeneração de inclusões muito delgadas, as quais obedecendo às leis não lineares da mecânica do dano, se degeneram dando origem a uma região de fratura. Para se atingir os objetivos descritos acima, foram desenvolvidas técnicas de integração analítica para o contorno e o domínio, bem como técnicas de condensação de domínios.

$\mathrm{Na}$ análise elástica de sólidos com multidomínios, foram impostas, antes de se escreverem as equações do sistema algébrico, a compatibilidade de deslocamentos e equilíbrio de forças. Tal técnica possibilitou, no primeiro caso de estudo, a eliminação das forças de superfície das interfaces como incógnitas do sistema global, formulação chamada neste trabalho de singular. Numa outra análise, denominada formulação hipersingular, se eliminou os deslocamentos da interface como incógnitas das equações. Tais formulações, como pode ser visto nos exemplos deste trabalho, se mostraram bastante precisas, notando-se que, à medida que se aumenta a rigidez da inclusão em relação ao domínio principal, a formulação singular apresenta resultados melhores que a formulação hipersingular, enquanto, à medida que a rigidez da inclusão se torna menor que a rigidez do sólido principal, a formulação hipersingular apresenta melhores resultados.

Foi estudado também o caso de concentração de tensões em cantos nas interfaces entre domínios. Estudos comparados com exemplos de formulação do MEF, mostram que para inclusões com rigidez tendendo ao infinito, a formulação hipersingular apresenta menor variação dos gradientes de força de superfície à medida 
que se chega ao canto.

No campo de inclusões muito delgadas foram utilizadas técnicas de condensação de domínios, para se evitar que o sistema algébrico final de equilíbrio apresente dependência linear, devido a pouca distância das faces da inclusão. Foi utilizada, primeiramente, a condensação do domínio $2 \mathrm{D}$ em fibra, possuindo esta rigidez a flexão zero e, posteriormente, a condensação em viga, obedecendo esta às hipóteses de Bernoulli. Em exemplos comparados de análise 2D via MEC com acoplamento MEC/MEF, ficou demonstrada a precisão de tais técnicas, além de notar uma diminuição das incógnitas do problema.

Foram feitas análises de inclusões muito delgadas com rigidez quase nula, para se simular o comportamento de uma fratura elástica. Utilizando-se técnicas da formulação singular e de condensação de domínio, conseguiu-se neste trabalho se discretizar inclusões muito finas, cujo comportamento se aproximou daquele da mecânica da fratura linear, como pode ser visto em exemplos comparados com outros métodos numéricos.

No capítulo de análise não-linear deste trabalho foram utilizados os modelos constitutivos de dano simples e dano do concreto. Para tal, foi adotada a técnica das tensões iniciais, que permite a análise de vasta gama de problemas não-lineares. Foi desenvolvida a técnica de degeneração de um domínio muito esbelto, o qual se degenerará segundo a lei não-linear de dano simples, dando origem ao início de abertura de uma fratura. Os exemplos, contudo, mostram que a formulação pode ser usada para se determinar uma área passível de um processo de fraturamento, e posteriormente, nessa área, deve ser utilizado elementos de fratura como elementos discretos e elementos de formulação dual.

Tanto para formulação linear quanto para não linear, foi necessário o desenvolvimento de técnicas de integração analítica para todos os elementos de contorno e para o domínio. Tais integrais apresentaram excelente precisão numérica, sendo comparadas com a técnica de subelementação. Tal precisão da técnica, só foi limitada pela própria precisão do compilador do programa de linguagem FORTRAN, entretanto, deve-se salientar que, se for utilizada uma precisão de softwares de análise matemática, a precisão aumenta substancialmente.

Como proposta para futuros desenvolvimentos, cita-se a necessidade de se 
aperfeiçoar o trabalho da degeneração de subdomínios muito esbeltos, regidos por leis constitutivas não lineares que considere a abertura física de possíveis sub-regiões neste domínio esbelto, que após degenerados darão origem a regiões de fratura. Os progressos neste campo devem-se situar na pesquisa da determinação do caminho das fraturas, e na determinação da sequência em que aparecerão prováveis zonas de danificação. 


\section{Referências Bibliográficas}

AGOSTINHO, P. C. P. (1998). Associação de chapas através da combinação dos métodos dos elementos de contorno e finitos considerando enrijecedores e crack coesivo. 172 f. Tese (Doutorado), Escola de Engenharia de São Carlos, Universidade de São Paulo, São Carlos - SP.

ALIABADI, M. H. ; BREBBIA, C. A. (1996). Boundary element formulation in fracture mechanics. In H. Nissitani; M. H. Aliabadi; I. S. Nishida ; D. J. Cartwright (Eds.), Localized damage IV (p. 3-20). Southampton, UK: Comput. Mech. Pub.

ALIABADI, M. H. ; ROOKE, D. P. (1991). Numerical Fracture Mechanics. Southampton, UK; Boston: Comp. Mech. Publications and Kluwer Academic Publishers, 276 p..

ALMEIDA, V. S. (2003). Análise da interação solo não-homogêneo/estrutura via acoplamento MEC/MEF. 192 f. Tese (Doutorado), Escola de Engenharia de São Carlos - Universidade de São Paulo, São Carlos - SP.

ALVARES, M. S. (1993). Estudo de um modelo de dano para o concreto : formulação, identificação paramétrica e aplicação com o emprego do método dos elementos finitos. 123 f. Dissertação (Mestrado), Escola de Engenharia de São Carlos - Universidade de São Paulo, São Carlos - SP.

ALVARES, M. S. (1999). Contribuição ao estudo e emprego de modelos simplificados de dano e plasticidade para a análise de estruturas de barras de concreto armado. 150 f. Tese (Doutorado), Escola de Engenharia de São Carlos - Universidade de São Paulo, São Carlos - SP.

BALBO, A. R. (1998). Contribuição à formulação matemática de modelos constitutivos para materiais com dano contínuo. 222 f. Tese (Doutorado), Escola de Engenharia de São Carlos - Universidade de São Paulo, São Carlos - SP. 
BANERJEE, P. K. (1981). The Boundary Element Methods in Engineering (2nd ed.). Berkshire, England: McGraw-Hill, 494 p..

BARBIRATO, J. C. C. (1999). Método dos elementos de contorno com a reciprocidade dual para a análise transiente tridimensional da mecânica do fraturamento. 212 f. Tese (Doutorado), Escola de Engenharia de São Carlos Universidade de São Paulo, São Carlos - SP.

BAREMBLATT, G. I. (1962). The mathematical theory of equilibrium cracks in brittle fracture. In Advances in Applied Mechanics 7 (p. 55-129). New York: Academic Press.

BARROS, F. B. (2002). Métodos sem malha e método dos elementos finitos generalizados em análise não-linear de estruturas. 172 f. Tese (Doutorado), Escola de Engenharia de São Carlos - Universidade de São Paulo, São Carlos - SP.

BENALLAL, A.; BOTTA, A. S. ; VENTURINI, W. S. (2006). On the description of localization and failure phenomena by the boundary element method. Computer Methods in Applied Mechanics and Engineering, Great Britain, n. 195, p. 5833-5856, mar.

BENALLAL, A. ; VENTURINI, W. S. (2004). Uma formulação do mec para a simulação numérica de descontinuidades fortes. Revista Internacional de Métodos Numéricos para Cálculo y Diseño en Ingeniería, Vol. 3, n. 20, p. 215-234.

BLANDFORD, G. E.; INGRAFFEA, A. R. ; LIGGET, J. A. (1981). Two dimensional stress intensity factor computations using the boundary element method. Int. J. Frac. Mech, Netherland, Vol. 17, n. 4, p. 387-404, aug.

BORDERIE, C. L.; PIJAUDIER-CABOT, G. ; MAZARS, J. (1992). The mathematical theory of equilibrium cracks in brittle fracture. In D. e. a. OWEN (Ed.), Proc. of Complas III, 6-10 April, 1992 (p. 55-129). Swansea: Pineridge Press. 
BOTTA, A. S. (1998). Cálculo de esforços e deslocamentos em estruturas reticuladas considerando-se a mecânica do dano para a modelagem do concreto armado . 98 f. Dissertação (Mestrado), Escola de Engenharia de São Carlos - Universidade de São Paulo, São Carlos - SP.

BOTTA, A. S. (2003). Método dos elementos de contorno para análise de corpos danificados com ênfase no fenômeno da localização de deformações. 170 f. Tese (Doutorado), Escola de Engenharia de São Carlos - Universidade de São Paulo, São Carlos - SP.

BOTTA, A. S. ; VENTURINI, W. S. (2005). Reinforced 2d domain analysis using bem and regularized bem/fem combination. Computer Modeling in Engineering and Sciences - CMES, Forsyte,USA, Vol. 8, n. 1, p. 15-28.

BOtTA, A. S.; Venturini, W. S. ; BenAllAL, A. (2005a). Bem applied to damage models emphasizing localization and associated regularization techniques. Engineering Analysis with Boundary Elements, Great Britain, Vol. 29, n. 8, p. 814-827, aug.

BOtTA, A. S.; VEnTURINI, W. S. ; BENAlLAL, A. (2005b). Bem applied to damage models with emphasis on the localization and regularization techniques. Latin American Journal of Solids and Structures, n. 2, p. 89-101.

BOWLES, J. E. (1988). Foundation Analysis and Design (4th ed.). Singapore: McGraw-Hill International Editions, 998 p..

BREBBiA, C. A. ; DOMingueZ, J. (1992). Boundary Elements An Introductory Course. Southampton,England: Computation Mechanics, 292 p..

BUSSAMRA, F. L. S. (1993). Equações constitutivas do concreto baseadas na mecânica do dano contínuo. 300 f. Dissertação (Mestrado), Escola Politécnica - Universidade de São Paulo, São Paulo - SP. 
CARMO, R. M. S. (2003). Determinação da rigidez de estruturas de pavimentos através dos métodos dos elementos de contorno e finitos. $145 \mathrm{f}$. Tese (Doutorado), Escola de Engenharia de São Carlos - Universidade de São Paulo, São Carlos - SP.

CARPINTERI, A. (1992). Meccanica dei Materiali e della Frattura. Bologna: Pitagora Editrice, 276 p..

CEDOLIN, L.; POLI, S. D. ; IORI, I. (1987). Tensile behaviour of concrete. Proc. ASCE, J. Eng. Mech., Washington,DC, Vol. 113, n. 3, p. 431-449, mar.

CEN, Z. ; MAIER, G. (1992). Bifurcations and instabilities in fracture of cohesivesoftening structures: A boundary element analysis. Fatigue Fract. Eng. Mater., Oxford, Vol. 15, n. 9, p. 911-928.

CHAVES, E. W. V. S. (1997). Análise de placas com variação de espessura através do método dos elementos de contorno. 171 f. Dissertação (Mestrado), Escola de Engenharia de São Carlos - Universidade de São Paulo, São Carlos - SP.

CODA, H. B. ; VENTURINI, W. S. (1999). On the coupling of 3d bem and fem frame model applied to elastodynamic analysis. Int. J. Solids Struct, Great Britain, Vol. 36, n. 31-32, p. 4789-4804, nov.

COMI, C. ; PEREGO, U. (2000). A bi-dissipative damage model for concrete with applications to dam engineering. In Ecomas 2000, Barcelona, Spain.

CRISFIELD, M. A. (1991). Non-linear finite element analysis of solids and structures. Chichester, New York: Wiley, 494 p..

CRUSE, T. A. (1970). Lateral constraint in a cracked, three-dimensional elastic body. Int. J. Frac. Mech, Netherlands, Vol. 6, n. 3, p. 326-328, sep. 
CRUSE, T. A. (1988). Boundary Element Analysis in Computational Fracture Mechanics. Dordrecht, Boston: Kluwer Academic Publishers, 162 p..

CRUSE, T. A. (2004). Bie fracture mechanics analysis 25 years of developments. Computation Mechanics, Heidelberg, Vol. 18, n. 1, p. 1-11, dec.

CRUSE, T. A. ; VANBUREN, W. (1971). Three-dimensional elastic stress analysis of a fractured specimen with an edge crack. Int. J. Frac. Mech, Netherlands, Vol. 1, n. 7, p. 1-15, mar.

DOLBOW, J. E.; MOES, N. ; BELYTSCHKO, T. (2001). An extended finite element method for modeling crack growth with frictional contact. Computer methods in applied mechanics and engineering, Great Britain, Vol. 190, n. 51, p. 6825-6846, oct.

DOLBOW, J. E. ; NADEAU, J. C. (2002). On the use of effective properties for the fracture analysis of microstructured materials. Engineering Fracture Mechanics, Great Britain, Vol. 69, n. 14-16, p. 1607-1634, sep.

DRIEMEIER, L. (1995). Considerações sobre a fadiga em metais e o comportamento do concreto sob solicitação cíclica. 120 f. Dissertação (Mestrado), Escola de Engenharia de São Carlos - Universidade de São Paulo, São Carlos - SP.

DRIEMEIER, L. (1999). Contribuição ao estudo da localização de deformações com modelos constitutivos de dano e plasticidade. $123 \mathrm{f}$. Tese (Doutorado), Escola de Engenharia de São Carlos - Universidade de São Paulo, São Carlos - SP.

FERNANDES, G. R. (1998). O método dos elementos de contorno aplicado à análise não-linear de placas . 178 f. Dissertação (Mestrado), Escola de Engenharia de São Carlos - Universidade de São Paulo, São Carlos - SP. 
FERNANDES, G. R. (2003). Análise não-linear de estruturas de pavimentos de edifícios através do método dos elementos de contorno. 272 f. Tese (Doutorado), Escola de Engenharia de São Carlos - Universidade de São Paulo, São Carlos - SP.

FERNANDES, G. R. ; VENTURINI, W. S. (2002). Non-linear boundary element analysis of plates applied to concrete slabs. Engineering analysis with Boundary Elements, Great Britain, n. 26, p. 169-181, feb.

FUDOLI, C. A. (1999). Formulação do Método dos Elementos de Contorno e Plasticidade com Gradiente. 136 f. Tese (Doutorado), Escola de Engenharia de São Carlos - Universidade de São Paulo, São Carlos - SP.

GONÇALVES, R. (2003). Análise de propagação de fissuras por fadiga em concreto pelo MEF mediante a mecânica do dano contínuo. $103 \mathrm{f}$. Dissertação (Mestrado), Escola de Engenharia de São Carlos - Universidade de São Paulo, São Carlos - SP.

GRAY, L. J. ; GILES, G. E. (1988). Application of the thin cavity method to shield calculations in electroplating. In C. A. Brebbia (Ed.), BEM X (p. 441-452). Southampton: Comput Mech. Pub.

GRAY, L. J.; MARTHA, L. F. ; INGRAFFEA, A. R. (1990). Hypersingular integrals in boundary element fracture analysis. Int. J. Numerical Methods in Engineering, Vol. 29, n. 6, p. 1135-1158.

GRIFFITH, A. A. (1921). The phenomena of rupture and flow in solids. Phil. Trans. Royal Society, London, n. A221, p. 163-167.

GUIGGIANI, M. (1994). Hypersingular formulation for boundary stress evaluation. Engineering Analysis with Boundary Element, Great Britain, Vol. 13, n. 2, p. $169-179$. 
HILlERBORG, A.; MODEER, M. ; PETERSON, P. E. (1976). Analysis of crack formation and crack growth in concrete by means of fracture mechanics and finite elements. Cem. Concr. Res., Great Britain, Vol. 6, n. 6, p. 773-782, nov.

IRWIN, G. R. (1957). Analysis of stresses and strains near the end of a crack transvering a plate. J. of Appl. Mech., ASME, Washington, DC, n. 24, p. 361364.

JIANG, Y. S. ; VENTURINI, W. S. (1998). Limit analysis of slope stability with boundary element methods. In IV world congress on computational mechanics, Buenos Aires, Argentina.

KACHANOV, L. M. (1958). Time of rupture process under creep condictions. Izvestia Akademii Nauk, USSR, n. 8, p. 26-31.

KANE, J. H. (1994). Boundary element analysis in engineering continuum mechanics (9th ed.). Englewood Cliffs, New Jersey, USA: Prentice-Hall, 674 p..

KOMORI, K. (2001). Simulation of shearing by node separation method. Computer Structures, Great Britain, Vol. 79, n. 2, p. 197-207, jan.

LEITE, L. G. S. (1998). Aplicação do Método dos Elementos de Contorno a Análise de Placas sobre Fundações Elásticas segundo a Teoria de Reissner. 150 f. Dissertação (Mestrado), Escola de Engenharia-Universidade Federal de Minas Gerais, Belo Horizonte - MG.

LEITE, L. G. S.; VEnTURINI, W. S. ; CODA, H. B. (2003). Two dimensional solids reinforced by thin bars using the boundary element method. Engineering Analysis with Boundary Element, Great Britain, Vol. 27, n. 3, p. 193-201, mar.

LEITE, L. G. S.; VENTURINI, W. S. ; CODA, H. B. (2005). Stiff and soft thin inclusions in two-dimensional solids by the boundary element method. Engineering 
Analysis with Boundary Element, Great Britain, Vol. 29, n. 3, p. 257-267, mar.

LEMAITRE, J. ; CHABOCHE, J. L. (1985). M?anics des materiaux solides. Bordas: Dunod, p..

LIANG, R. Y. ; LI, Y. N. (2004). Simulations of non-linear fracture process zone in cementitious material a boundary element approach. Computational Mechanics, Heidelberg, Vol. 7, n. 6, p. 413-427, dec.

LOPES, M. C. (1996). Modelagem numérica do crescimento de fraturas através do método dos elementos de contorno. 261 f. Dissertação (Mestrado), Escola de Engenharia de São Carlos - Universidade de São Paulo, São Carlos - SP.

LOVÓN, O. B. R. (2006). Formulação h-Adaptativa do método dos elementos de contorno para elasticidade bidimensional com ênfase na propagação da fratura. 122 f. Tese (Doutorado), Escola de Engenharia de São Carlos Universidade de São Paulo, São Carlos - SP.

MACIEL, D. N. (2003). Determinação dos fatores de intensidade de tensão estáticos e dinâmicos via MEC com integração analítica em coordenadas locais. 125 f. Dissertação (Mestrado), Escola de Engenharia de São Carlos Universidade de São Paulo, São Carlos - SP.

MAIER, G.; NOVATI, G. ; CEN, Z. (1993). Symmetric galerkin boundary element method for quasi-brittle and frictional contact problems. Comp. Mech., Heidelberg, Vol. 13, n. 1, p. 74-89, nov.

MANZOLI, O. L. (1998). Un modelo anlítico y numérico para la simulación de discontinuidades fuertes en la mecánica de sólidos. 231 f. Tesis doctoral, Universitat Politècnica de Catalunya, Barcelona - España.

MAZARS, J. (1984). Application de la mécanique de l'endommagement au 
comportement nonlinéare et à la rupture du béton de structure. f. Dr. of science thesis, University of Paris 6, Cachan - France.

MAZARS, J. (1986). Description of micro and macroscale damage of concrete structures. Eng. Fracture Mechanics, Great Britain, Vol. 25, n. 6, p. 729-737.

MAZARS, J. ; PIJAUDIER-CABOT, G. (1995). Continuum damage theory application to concrete. J. Eng. Mech. ASCE, Berkshire, England, Vol. 5, n. 5, p. $345-365$.

MENDONÇA, A. V. (1997). Análise da interação placa-estaca-solo via combinação do método dos elementos finitos com o método dos elementos de contorno. 151 f. Dissertação (Mestrado), Escola de Engenharia de São Carlos Universidade de São Paulo, São Carlos - SP.

OSHIMA, S. T. (2004). Uma combinação MEC/MEF para análise da interação de estacas inclinadas e o solo. 84 f. Dissertação (Mestrado), Escola de Engenharia de São Carlos - Universidade de São Paulo, São Carlos - SP.

OWEN, D. R. J. ; HINTON, E. (1980). Finite Elements in Plasticity - Theory and Pratice. Swansea, U. K.: McGraw-Hill, 594 p..

PACCOLA, R. R. (2004). Análise não linear física de placas e cascas anisotrópicas laminadas acopladas ou não com meio contínuo tridimensional viscoelástico através da combinação entre o MEC e o MEF. 192 f. Tese (Doutorado), Escola de Engenharia de São Carlos - Universidade de São Paulo, São Carlos - SP.

PEREIRA, J. P. A. (2004). Extração de fatores de intensidade de tensão utilizando a solução do método dos elementos finitos generalizados. $160 \mathrm{f}$. Dissertação (Mestrado), Escola de Engenharia de São Carlos - Universidade de São Paulo, São Carlos - SP. 
PITUBA, J. J. C. (1998). Estudo e aplicação de modelos constitutivos para o concreto fundamentados na mecânica do dano contínuo . 130 f. Dissertação (Mestrado), Escola de Engenharia de São Carlos - Universidade de São Paulo, São Carlos - SP.

PITUBA, J. J. C. (2003). Sobre a formulação de um modelo de dano para o concreto. 151 f. Tese (Doutorado), Escola de Engenharia de São Carlos - Universidade de São Paulo, São Carlos - SP.

PORTElA, A.; ALIABADI, M. H. ; ROOKE, D. P. (1992). The dual boundary element method: effective implementation for crack problems. Int. J. Numerical Methods in Engineering, Vol. 33, n. 6, p. 1269-1287, apr.

RIBEIRO, D. B. (2005). Análise da interação solo-estrutura via acoplamento MEC-MEF. 120 f. Dissertação (Mestrado), Escola de Engenharia de São Carlos Universidade de São Paulo, São Carlos - SP.

RUDOLPHI, T. J.; KRISHNASAMY, G.; SCCHMERR, L. W. ; RIZZO, P. J. (1988). Use of strongly singular integral equations for crack problems. In C. A. Brebbia (Ed.), BEM X (p. 249-264). Southampton: Comput Mech. Pub.

SCHOLLMAN, M.; FULLAND, M. ; RICHARD, H. A. (2003). Development of a new software for adaptative crack growth simulations in 3d structures. Engineering Fracture Mechanics, Great Britain, Vol. 70, n. 2, p. 249-268, jan.

SILVA, N. A. (1996). Aplicação do Método dos Elementos de Contorno à análise de Placas com Enrijecedores utilizando a Teoria de Reissner. 162 f. Tese (Doutorado), Escola de Engenharia de São Carlos - Universidade de São Paulo, São Carlos - SP.

SUKUMAR, N.; CHOPP, D. L. ; MORAN, B. (2003). Extended finite element method and fast marching method for three-dimensional fatigue crack propagation. 
Engineering Fracture Mechanics, Great Britain, Vol. 70, n. 1, p. 29-48.

TORRES, I. F. R. (2003). Desenvolvimento e aplicação do método dos elementos finitos generalizados em análise tridimensional não-linear de sólidos. 221 f. Tese (Doutorado), Escola de Engenharia de São Carlos - Universidade de São Paulo, São Carlos - SP.

VANALLI, L. (2004). O MEC e o MEF aplicados à análise de problemas viscoplásticos em meios anisotrópicos e compostos. 194 f. Tese (Doutorado), Escola de Engenharia de São Carlos - Universidade de São Paulo, São Carlos - SP.

VENTURINI, W. S. (1992). Alternative formulations of the boundary element method for potential and elastic zoned problems. Engineering Analysis, Great Britain, Vol. 9, n. 3, p. 203-207, feb.

WATSON, J. O. (1986). Hermitian cubic and singular elements for plane strain. In P. K. Banerjee ; J. O. Watson (Eds.), Developments in boundary elements 4 (p. 1-28). London: Elsevier Applied Science Publishers.

WUTZOW, W. W. (2003). Formulação do método dos elementos de contorno para análise de chapas com enrijecedores. 140 f. Dissertação (Mestrado), Escola de Engenharia de São Carlos - Universidade de São Paulo, São Carlos - SP.

YUANHAN, W. (1991). Asymmetric crack problems calculated by the boundary collocation method. Engineering Fract. Mechanics, Great Britain, Vol. 40, n. 1, p. $133-143$. 